\title{
Cu-Catalyzed Direct C6-Arylation of Indoles
}

\section{(Supporting Information)}

Youqing Yang, ${ }^{\dagger}$ Ruirui Li,${ }^{\dagger}$ Yue Zhao, ${ }^{\dagger}$ Dongbing Zhao, ${ }^{\ddagger}$ and Zhuangzhi Shi* ${ }^{\dagger}$

${ }^{\dagger}$ State Key Laboratory of Coordination Chemistry, Collaborative Innovation Center of Chemistry for Life Sciences, School of Chemistry and Chemical Engineering, Nanjing University, Nanjing, 210093, China

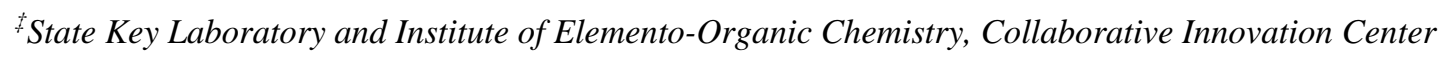
of Chemical Science and Engineering, Nankai University, Tianjin, 300071, China E-mail: shiz@nju.edu.cn

\section{Table of Contents}

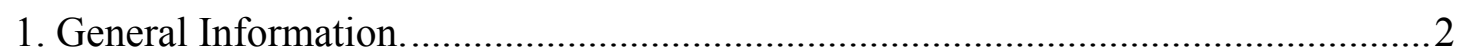

2. General Procedure for Synthesis of Starting Materials................................................

3. General Procedure for C6-Selective C-H Arylation of Indoles...............................

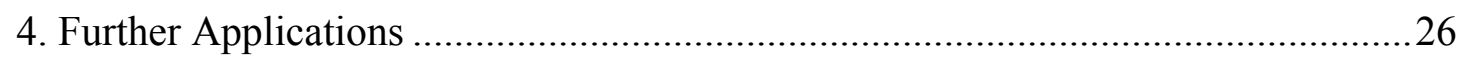

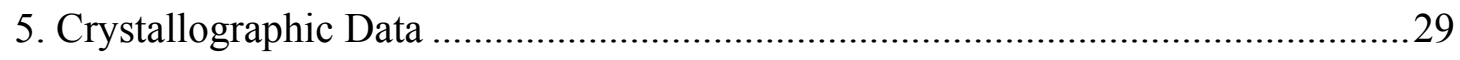

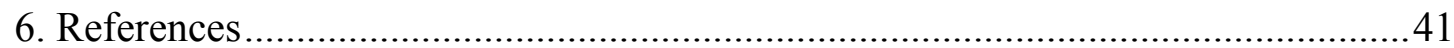

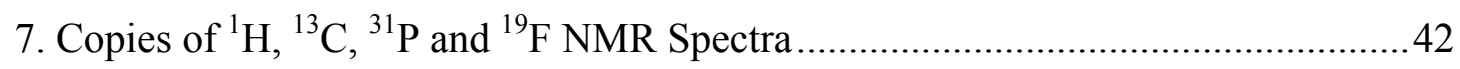




\section{General Information.}

Unless otherwise noted, all reactions were performed under an argon atmosphere usingflame-dried glassware. Toluene, DCM and DCE were distilled over $\mathrm{CaH}_{2}$. All new compounds were fully characterized. NMR-spectra were recorded on Bruker AV-300, ARX-400 MHz or a ARX-600Associated. ${ }^{1} \mathrm{H}$ NMR spectra datawere reported as $\delta$ values in ppm relative to chloroform $(\delta 7.26)$ if collected in $\mathrm{CDCl}_{3} \cdot{ }^{13} \mathrm{C}$ NMR spectra data were reported as $\delta$ values in ppm relative to chloroform $(\delta 77.00)$ if collected in $\mathrm{CDCl}_{3}$ (the carbon attached to B was not observed). ${ }^{1} \mathrm{H}$ NMR coupling constants were reported in $\mathrm{Hz}$, and multiplicity was indicated as follows: s (singlet); $\mathrm{d}$ (doublet); $\mathrm{t}$ (triplet); q (quartet); quint (quintet); m (multiplet);dd (doubletof doublets); ddd (doublet of doublet of doublets); dddd (doublet of doublet of doublet of doublets); dt (doublet of triplets); td (triplet of doublets); ddt (doublet of doublet of triplets); dq (doubletof quartets); app (apparent); br (broad). Mass spectra were conducted at Micromass Q-Tof instrument (ESI) and Agilent Technologies 5973N (EI). All reactions were carried out in flame-dried $25-\mathrm{mL}$ Schlenk tubes with Teflon screw caps under argon. $\mathrm{CuO}$ was purchased from J\&K. All diaryliodonium salts are known substrates, which were prepared according to reference $1 . N-\mathrm{P}(\mathrm{O})^{t} \mathrm{Bu}_{2}$ indoles $\mathbf{1 a}-\mathbf{1 e}, \mathbf{1 h}, \mathbf{1 n}, \mathbf{1 i}, \mathbf{1 j}, \mathbf{1}$ and $\mathbf{1 0}$ are known substrates, which were prepared according to reference 2 . 


\section{General Procedure for Synthesis of Starting Materials}

\section{Typical procedure:}

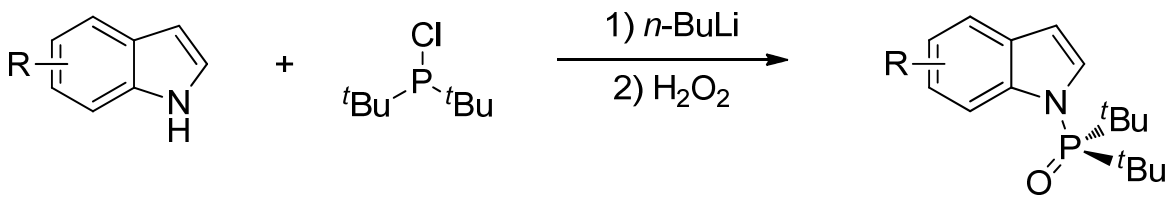

According to the literature, ${ }^{2}$ to a solution of indole (5.0 mmol, 1.0 equiv) in 10 $\mathrm{ml}$ anhydrous THF at $0{ }^{\circ} \mathrm{C}$ was added a solution of $n$-BuLi (solution in hexanes, 6.0 mmol, 1.2 equiv) dropwise. After stirring for $15 \mathrm{~min}$, di-tert-butylchlorophosphine ( $6.0 \mathrm{mmol}, 1.2$ equiv) was added dropwise. The mixture was allowed to stir and warm to room temperature over several hours. After addition of $2 \mathrm{~mL} \mathrm{MeOH}$, most of the solvent was removed under reduced pressure and the residue was suspended in $30 \mathrm{ml}$ $\mathrm{MeOH}$ and cooled to $0{ }^{\circ} \mathrm{C}$. Slow addition of excess $\mathrm{H}_{2} \mathrm{O}_{2}(1 \mathrm{ml}$ of $30 \%$ solution, approx. $8 \mathrm{mmol}$ ) caused the suspended material to dissolve over a $30 \mathrm{~min}$ period resulting in a light yellow solution. After adding $8 \mathrm{~mL} \mathrm{Na}_{2} \mathrm{SO}_{3}$ (2 $\mathrm{M}$ solution) dropwise, the solution was stirred for $2 \mathrm{~h}$, allowed to warm to room temperature, treated with $12 \mathrm{ml} \mathrm{HCl}$ (10\% solution), and stirred for another hour. Most of the $\mathrm{MeOH}$ was removed under reduced pressure, and the remaining residue was poured into $40 \mathrm{ml} \mathrm{H} \mathrm{H}_{2} \mathrm{O}$ and extracted with DCM $(3 \times 25 \mathrm{ml})$. The combined organics were dried $\left(\mathrm{Na}_{2} \mathrm{SO}_{4}\right)$ and evaporated to give an orange solid. Further purification was carried out by flash chromatography $(\mathrm{DCM}: \mathrm{MeOH}=100: 1)$.

\section{Di-tert-butyl(4-fluoro-1H-indol-1-yl)phosphine oxide (1f)}

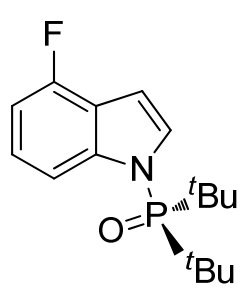

$1 f$

According to typical procedure, compound 1f was prepared from 4-fluoro- $1 \mathrm{H}$-indole and di-tert-butylchlorophosphine in 94\% yield as a white solid. ${ }^{1} \mathbf{H}$ NMR (400 $\left.\mathbf{M H z}, \mathbf{C D C l}_{3}\right) \delta 8.30(\mathrm{~d}, J=8.5 \mathrm{~Hz}$, $1 \mathrm{H}), 7.21(\mathrm{dd}, J=3.4,1.1 \mathrm{~Hz}, 1 \mathrm{H}), 7.14(\mathrm{td}, J=8.2,5.7 \mathrm{~Hz}, 1 \mathrm{H})$, $6.84(\mathrm{dd}, J=9.9,8.0 \mathrm{~Hz}, 1 \mathrm{H}), 6.80-6.75(\mathrm{~m}, 1 \mathrm{H}), 1.34(\mathrm{~d}, J=14.8$ $\mathrm{Hz}, 18 \mathrm{H}) .{ }^{13} \mathbf{C}$ NMR (101 MHz, $\left.\mathbf{C D C l}_{3}\right) \delta 155.75$ (d, $\left.J=247.5 \mathrm{~Hz}\right), 143.83$ (d, $J=$ $9.4 \mathrm{~Hz}), 126.24$ (d, $J=4.8 \mathrm{~Hz}), 123.89$ (d, $J=7.3 \mathrm{~Hz}), 118.43$ (dd, $J=21.9,5.8 \mathrm{~Hz})$, 
$112.32(\mathrm{~d}, J=4.0 \mathrm{~Hz}), 106.35(\mathrm{~d}, J=18.1 \mathrm{~Hz}), 102.81(\mathrm{~d}, J=5.0 \mathrm{~Hz}), 38.64$ (d, $J=$ 68.1 Hz), 26.64. ${ }^{31}$ P NMR (162 MHz, CDCl 3 ) $\delta 63.51 .{ }^{19}$ F NMR (376 MHz, $\mathbf{C D C l}_{3}$ ) $\delta$-122.78. ATR-FTIR (cm $\left.{ }^{-1}\right): 3120,1622,1481,1400,1283,1124,1032,815,761$, 648. HRMS m/z (ESI): calcd for $\mathrm{C}_{16} \mathrm{H}_{24} \mathrm{FNOP}(\mathrm{M}+\mathrm{H})^{+}$296.1574, found 296.1576.

\section{(4-Bromo-1H-indol-1-yl)di-tert-butylphosphine oxide (1j)}

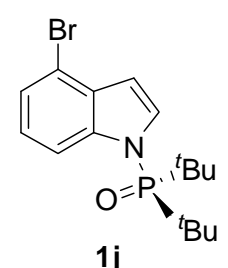

According to typical procedure, compound $\mathbf{1 j}$ was prepared from 4-bromo- $1 \mathrm{H}$-indole and di-tert-butylchlorophosphine in $88 \%$ yield as a white solid. ${ }^{1} \mathbf{H}$ NMR (400 MHz, $\left.\mathbf{C D C l}_{3}\right) \delta 8.49(\mathrm{~d}, J=8.5 \mathrm{~Hz}, 1 \mathrm{H})$, $7.34(\mathrm{dd}, J=7.6,0.6 \mathrm{~Hz}, 1 \mathrm{H}), 7.28(\mathrm{dd}, J=3.5,1.4 \mathrm{~Hz}, 1 \mathrm{H}), 7.13-$ $6.99(\mathrm{~m}, 1 \mathrm{H}), 6.75(\mathrm{ddd}, J=3.3,2.1,0.6 \mathrm{~Hz}, 1 \mathrm{H}), 1.33$ (d, $J=14.8 \mathrm{~Hz}, 18 \mathrm{H}) .{ }^{13} \mathrm{C}$ NMR (101 MHz, CDCl $\left.{ }_{3}\right) \delta$ 141.78, 129.89 (d, $\left.J=5.3 \mathrm{~Hz}\right), 126.89$ (d, $\left.J=4.5 \mathrm{~Hz}\right)$, 124.49, 124.40, 115.39, 113.83, 107.18 (d, $J=4.5 \mathrm{~Hz}), 38.63$ (d, $J=68.6 \mathrm{~Hz}), 26.61$. ${ }^{31}$ P NMR (162 MHz, CDCl $\left.\mathbf{~}_{3}\right) \delta$ 63.82. ATR-FTIR (cm $\left.{ }^{-1}\right): 3134,1684,1401,1279$, 1151, 1133, 1092, 999, 886, 814, 759, 665, 617. HRMS m/z (ESI): calcd for $\mathrm{C}_{16} \mathrm{H}_{23} \mathrm{BrNOPNa}^{+}$378.0593, found 378.0595.

\section{Methyl 1-(di-tert-butylphosphoryl)-1H-indole-3-carboxylate (1I)}

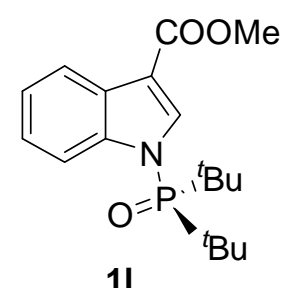

According to typical procedure, compound $\mathbf{1 l}$ was prepared from methyl $1 H$-indole-3-carboxylate and di-tert-butylchlorophosphine in 66\% yield as a white solid. ${ }^{1} \mathbf{H}$ NMR (400 $\left.\mathbf{~ M H z , ~} \mathbf{C D C l}_{3}\right) \delta 8.59-$ $8.47(\mathrm{~m}, 1 \mathrm{H}), 8.22-8.07(\mathrm{~m}, 1 \mathrm{H}), 7.93(\mathrm{~d}, J=2.0 \mathrm{~Hz}, 1 \mathrm{H}), 7.33-$ 7.27 (m, 2H), 3.94 (s, 3H), 1.37 (d, J = 15.0 Hz, 18H). ${ }^{13} \mathbf{C}$ NMR (101 MHz, CDCl $\left.\mathbf{~}_{3}\right)$ $\delta 164.99,142.21,132.59$ (d, $J=4.7 \mathrm{~Hz}), 126.44$ (d, $J=4.3 \mathrm{~Hz}), 124.49,123.22$, 120.91, 116.55, $112.53(\mathrm{~d}, J=4.6 \mathrm{~Hz}), 51.40,38.71(\mathrm{~d}, J=67.8 \mathrm{~Hz}), 26.65 .{ }^{31} \mathbf{P}$ NMR (162 MHz, $\left.\mathbf{C D C l}_{3}\right) \delta$ 65.49. ATR-FTIR (cm $\left.{ }^{-1}\right): 3131,1703,1536,1400,1309,1282$, 1177, 1136, 1065, 1015, 985, 918, 814, 783, 762, 664, 607. HRMS m/z (ESI): calcd for $\mathrm{C}_{18} \mathrm{H}_{26} \mathrm{NO}_{3} \mathrm{PNa}^{+} 358.1543$, found 358.1545 . 


\section{Methyl 4-(1-(di-tert-butylphosphoryl)-1H-indol-3-yl)butanoate (1n)}

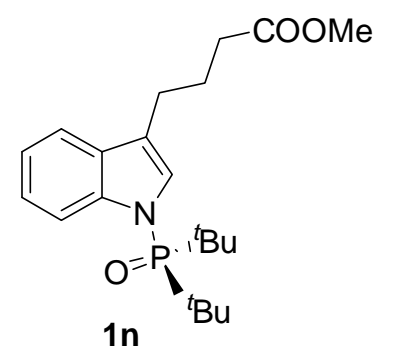

According to typical procedure, compound 1n was prepared from methyl 4-(1H-indol-3-yl)butanoate and di-tert-butylchlorophosphine in $79 \%$ yield as a white solid. ${ }^{1} \mathbf{H}$ NMR (400 MHz, $\left.\mathbf{C D C l}_{3}\right) \delta 8.49(\mathrm{~d}, J=7.9 \mathrm{~Hz}, 1 \mathrm{H}), 7.52(\mathrm{~d}$, $J=7.4 \mathrm{~Hz}, 1 \mathrm{H}), 7.25-7.09(\mathrm{~m}, 2 \mathrm{H}), 7.00(\mathrm{~s}, 1 \mathrm{H}), 3.67$ (s, 3H), $2.79(\mathrm{t}, J=7.2 \mathrm{~Hz}, 2 \mathrm{H}), 2.38(\mathrm{t}, J=7.1 \mathrm{~Hz}, 2 \mathrm{H}), 2.17-1.99(\mathrm{~m}, 2 \mathrm{H}), 1.33(\mathrm{~d}, J=$ $14.6 \mathrm{~Hz}, 18 \mathrm{H}) .{ }^{13} \mathbf{C}$ NMR (101 MHz, $\left.\mathbf{C D C l}_{3}\right) \delta 173.90,142.19,129.29,123.52$, $123.38(\mathrm{~d}, J=4.2 \mathrm{~Hz}), 121.10,119.65(\mathrm{~d}, J=4.4 \mathrm{~Hz}), 118.24,116.40,51.52,38.55(\mathrm{~d}$, $J=69.4 \mathrm{~Hz}$ ), 33.48, 26.74, 24.78, 24.34. ${ }^{31} \mathbf{P}$ NMR (162 $\left.\mathbf{M H z}, \mathbf{C D C l}_{3}\right) \delta 62.22$. ATR-FTIR (cm $\left.{ }^{-1}\right): 3135,1733,1686,1401,1310,1286,1216,1151,1118,967,815$, 759, 662, 616. HRMS m/z (ESI): calcd for $\mathrm{C}_{21} \mathrm{H}_{32} \mathrm{NO}_{3} \mathrm{PNa}^{+}$400.2012, found 400.2014 .

Di-tert-butyl(3-chloro-1H-indol-1-yl)phosphine oxide (1h) and Di-tert-butyl(2,3-dichloro-1H-indol-1-yl)phosphine oxide (1i)

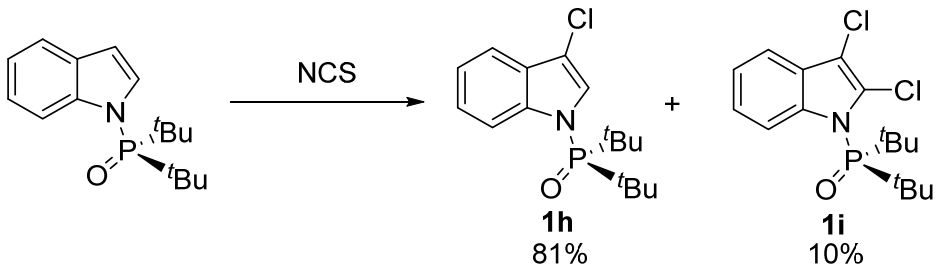

To a solution of di-tert-butyl(1H-indol-1-yl)phosphine oxide $(554.7 \mathrm{mg}, 2.0$ $\mathrm{mmol})$ and 2,4,6- trimethylaniline $(2.7 \mathrm{mg}, 10 \mathrm{~mol} \%)$ in dry acetonitrile $(0.25 \mathrm{M})$ at room temperature under $\mathrm{N}_{2}$ atmosphere was added $N$-chlorosuccinimide (NCS) (320.5 mg, 2.4 mmol) with stirring. The reaction was monitored by thin layer chromatography. Upon consumption of starting material the crude reaction mixture was concentrated and directly purified by silica gel flash column chromatography (hexane/ethyl acetate $=30: 1)$ giving indole $\mathbf{1 h}(81 \%)$ and $\mathbf{1 i}(10 \%)$, respectively. Indole lh. ${ }^{1} \mathbf{H}$ NMR (400 MHz, $\left.\mathbf{C D C l}_{3}\right) \delta 8.55-8.48(\mathrm{~m}, 1 \mathrm{H}), 7.63-7.53(\mathrm{~m}, 1 \mathrm{H})$, $7.31-7.26(\mathrm{~m}, 1 \mathrm{H}), 7.24(\mathrm{dd}, J=7.1,1.3 \mathrm{~Hz}, 1 \mathrm{H}), 7.21(\mathrm{~d}, J=1.5 \mathrm{~Hz}, 1 \mathrm{H}), 1.35$ (d, $J$ $=14.8 \mathrm{~Hz}, 18 \mathrm{H}) .{ }^{13} \mathbf{C}$ NMR (101 MHz, $\left.\mathbf{C D C l}_{3}\right) \delta 140.74,126.67(\mathrm{~d}, J=4.5 \mathrm{~Hz})$, 
124.67, 122.44 (d, $J=4.7 \mathrm{~Hz}), 122.13,117.77,116.55,111.37$ (d, $J=5.0 \mathrm{~Hz}), 38.64$ $(\mathrm{d}, \mathrm{J}=68.5 \mathrm{~Hz}), 26.65 .{ }^{31} \mathbf{P}$ NMR (162 MHz, $\left.\mathbf{C D C l}_{3}\right) \delta 64.18$. ATR-FTIR $\left(\mathbf{c m}^{-1}\right)$ : 3161, 1684, 1401, 1278, 1198, 1109, 1042, 940, 814, 756, 705, 661, 606. HRMS m/z (ESI): calcd for $\mathrm{C}_{16} \mathrm{H}_{23} \mathrm{ClNOPNa}^{+}$334.1098, found 334.1100. Indole li. ${ }^{1} \mathbf{H}$ NMR (400 MHz, $\left.\mathbf{C D C l}_{3}\right) \delta 8.80-8.71(\mathrm{~m}, 1 \mathrm{H}), 7.48(\mathrm{dd}, J=5.9,3.4 \mathrm{~Hz}, 1 \mathrm{H}), 7.25(\mathrm{dd}, J$ $=6.5,3.3 \mathrm{~Hz}, 2 \mathrm{H}), 1.43(\mathrm{~d}, J=15.6 \mathrm{~Hz}, 18 \mathrm{H}) \cdot{ }^{13} \mathbf{C}$ NMR (101 MHz, $\left.\mathbf{C D C l}_{3}\right) \delta$ 140.64, $126.81(\mathrm{~d}, J=3.7 \mathrm{~Hz}), 124.69,122.63,120.00(\mathrm{~d}, J=1.8 \mathrm{~Hz}), 117.99$, $117.13,111.12,40.70$ (d, $J=64.0 \mathrm{~Hz}), 27.16 .{ }^{31} \mathbf{P}$ NMR (162 MHz, $\left.\mathbf{C D C l}_{3}\right) \delta 74.97$. ATR-FTIR (cm $\left.\mathbf{c m}^{-1}\right): 3122,1684,1401,1195,1063,943,810,755,713,657,615$. HRMS m/z (ESI): calcd for $\mathrm{C}_{16} \mathrm{H}_{22} \mathrm{Cl}_{2} \mathrm{NOPNa}^{+}$368.0708, found 368.0710.

\section{Di-tert-butyl(3-iodo-1H-indol-1-yl)phosphine oxide (1k)}
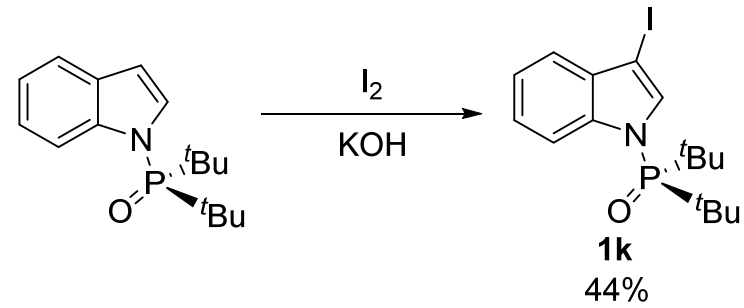

$\mathrm{I}_{2}(1.015 \mathrm{~g}, 4.0 \mathrm{mmol}, 2.00$ equiv) was added in one pot to a solution of di-tert-butyl(1H-indol-1-yl)phosphine oxide (554.26 mg, $2.0 \mathrm{mmol}, 1.00$ equiv) and $\mathrm{KOH}$ (420.75 mg, $7.5 \mathrm{mmol}, 3.75$ equiv) in DMF $(6 \mathrm{~mL})$ at rt. The reaction was stirred at the same temperature for 10 minutes then poured into a suspension of ice and water $(40 \mathrm{~mL})$ containing ammonia $(0.5 \%)$ and sodium metabisulphite $(0.1 \%)$. The emulsion was then extracted with a solution of hexane/ethyl acetate 1:1 $(5 \times 100$ $\mathrm{mL}$ ). The organic phase was washed with cold water, dried over $\mathrm{Na}_{2} \mathrm{SO}_{4}$, evaporated under reduced pressure and and purified by silica gel flash column chromatography (hexane/ethyl acetate $=3: 1$ ) giving compound $\mathbf{1 k}$ in $48 \%$ yield as a white solid. ${ }^{1} \mathbf{H}$ NMR (400 MHz, CDCl $) \delta 8.57-8.36(\mathrm{~m}, 1 \mathrm{H}), 7.41(\mathrm{ddd}, J=5.3,2.6,1.4 \mathrm{~Hz}, 1 \mathrm{H})$, $7.30(\mathrm{t}, J=1.8 \mathrm{~Hz}, 1 \mathrm{H}), 7.27(\mathrm{dd}, J=7.0,3.3 \mathrm{~Hz}, 2 \mathrm{H}), 1.34(\mathrm{~d}, J=14.9 \mathrm{~Hz}, 18 \mathrm{H}) .{ }^{13} \mathrm{C}$ NMR (101 MHz, $\left.\mathbf{C D C l}_{3}\right) \delta 141.16,130.87$ (d, $\left.J=5.2 \mathrm{~Hz}\right), 129.95$ (d, $\left.J=4.2 \mathrm{~Hz}\right)$, 124.60, 122.38, 120.68, 116.34, 64.33 (d, $J=3.8 \mathrm{~Hz}), 38.67$ (d, $J=68.5 \mathrm{~Hz}), 26.66$. ${ }^{31}$ P NMR (162 MHz, CDCl M $\delta$ 64.10. ATR-FTIR (cm ${ }^{-1}$ ): 3143, 2966, 1601, 1400, 
1272, 1224, 1178, 1130, 1015, 920, 817, 757, 678, 663, 601. HRMS m/z (ESI): calcd for $\mathrm{C}_{16} \mathrm{H}_{23} \mathrm{INOPNa}{ }^{+}$426.0454, found 426.0453 .

\section{(E)-Di-tert-butyl(4-styryl-1H-indol-1-yl)phosphine oxide (1m)}

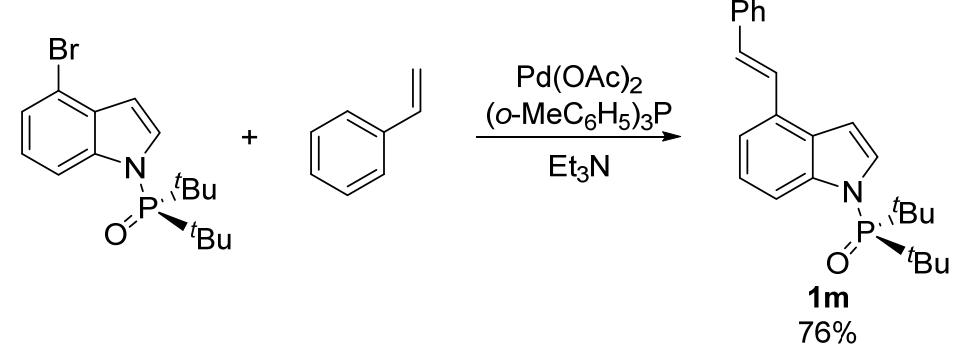

To a solution of (4-bromo-1H-indol-1-yl)di-tert-butylphosphine oxide (712.5 mg, $2.0 \mathrm{mmol})$ in triethylamine $(1.5 \mathrm{~mL})$ was added styrene $(458 \mu \mathrm{L}, 4.0 \mathrm{mmol})$, $\mathrm{Pd}(\mathrm{OAc})_{2}(18 \mathrm{mg}, 4 \mathrm{~mol} \%)$ and tris(o-tolyl)phosphine $(61 \mathrm{mg}, 10 \mathrm{~mol} \%)$ at room temperature. The reaction mixture was stirred for $2 \mathrm{~d}$ at $110^{\circ} \mathrm{C}$. The reaction mixture was filtered, and then the filtrate was poured into water and the resulting mixture was extracted with EtOAc. The organic layer was washed sequentially with aqueous saturated ammonium chloride solution, water and saturated brine, dried over anhydrous sodium sulfate and concentrated. The residue was purified by column chromatography on silica gel $(n$-hexane/EtOAc $=8: 1)$ affording $1 \mathbf{m}$ in $76 \%$ yield as a white solid. ${ }^{1} \mathbf{H}$ NMR (400 $\left.\mathbf{M H z}, \mathbf{C D C l}_{3}\right) \delta 8.48(\mathrm{~d}, J=8.4 \mathrm{~Hz}, 1 \mathrm{H}), 7.58(\mathrm{~d}, J=7.4$ $\mathrm{Hz}, 2 \mathrm{H}), 7.48(\mathrm{dd}, J=14.8,11.9 \mathrm{~Hz}, 2 \mathrm{H}), 7.38$ (t, $J=7.6 \mathrm{~Hz}, 2 \mathrm{H}), 7.33-7.18$ (m, 4H), $7.02-6.89(\mathrm{~m}, 1 \mathrm{H}), 1.36(\mathrm{~d}, J=14.7 \mathrm{~Hz}, 18 \mathrm{H}) .{ }^{13} \mathbf{C} \mathbf{N M R}\left(101 \mathbf{M H z}, \mathbf{C D C l}_{3}\right) \delta$ 142.05, 137.82, 129.42, 129.27, 128.65, 127.81 (d, $J=5.4 \mathrm{~Hz}), 127.44,126.61$ (d, $J=$ $5.0 \mathrm{~Hz}), 126.55,126.47,123.74,118.62,115.71,105.32$ (d, $J=5.0 \mathrm{~Hz}), 38.66$ (d, $J=$ 69.3 Hz), 26.71. ${ }^{31} \mathbf{P}$ NMR (162 $\left.\mathbf{M H z}, \mathbf{C D C l}_{3}\right) \delta$ 63.00. ATR-FTIR (cm $\left.{ }^{-1}\right): 3132$, 1685, 1400, 1271, 1166, 1141, 956, 815, 763, 747, 688, 660, 622. HRMS m/z (ESI): calcd for $\mathrm{C}_{24} \mathrm{H}_{30} \mathrm{NOPNa}^{+} 402.1957$, found 402.1960 . 


\section{(3,3'-Methylenebis(1H-indole-3,1-diyl))bis(di-tert-butylphosphine oxide) (10)}
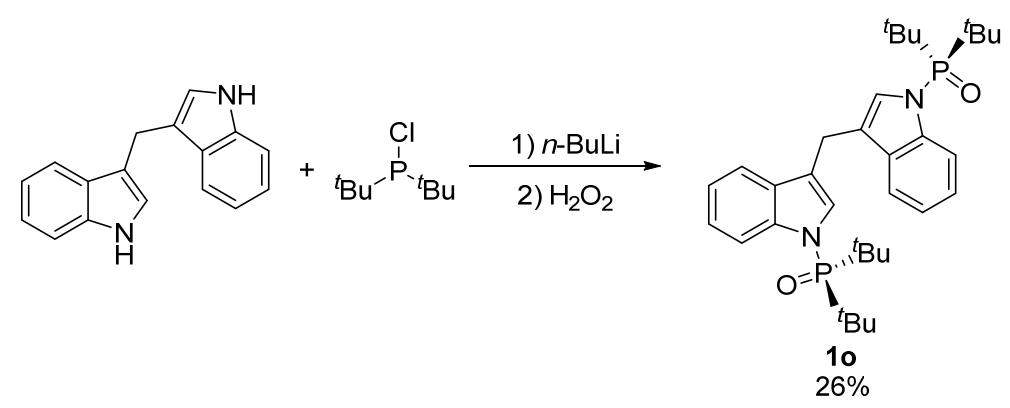

To a solution of $\operatorname{di}(1 H$-indol-3-yl)methane $(6.0 \mathrm{mmol}, 1.48 \mathrm{~g})$ in $12 \mathrm{ml}$ anhydrous THF at $0{ }^{\circ} \mathrm{C}$ was added a solution of $n$-BuLi (solution in hexane $2.5 \mathrm{M}$, $14.4 \mathrm{mmol}, 5.8 \mathrm{~mL}$ ) dropwise. After stirring for $15 \mathrm{~min}$, di-tert-butylchlorophosphine (14.4 mmol, $2.8 \mathrm{~mL}$ ) was added dropwise. The mixture was allowed to stir and warm to room temperature over several hours. After addition of $2.4 \mathrm{~mL} \mathrm{MeOH}$, most of the solvent was removed under reduced pressure and the residue was suspended in $18 \mathrm{ml}$ $\mathrm{MeOH}$ and cooled to $0{ }^{\circ} \mathrm{C}$. Slow addition of excess $\mathrm{H}_{2} \mathrm{O}_{2}(1 \mathrm{ml}$ of $30 \%$ solution, approx. $19.2 \mathrm{mmol}$ ) caused the suspended material to dissolve over a $30 \mathrm{~min}$ period resulting in a light yellow solution. After adding $14 \mathrm{~mL} \mathrm{Na} \mathrm{SO}_{3}$ (2 $\mathrm{M}$ solution) dropwise, the solution was stirred for $2 \mathrm{~h}$, allowed to warm to room temperature, treated with $14 \mathrm{ml} \mathrm{HCl}(10 \%$ solution), and stirred for another hour. Most of the $\mathrm{MeOH}$ was removed under reduced pressure, and the remaining residue was poured into $30 \mathrm{ml} \mathrm{H}_{2} \mathrm{O}$ and extracted with DCM $(3 \times 20 \mathrm{ml})$. The combined organics were dried $\left(\mathrm{Na}_{2} \mathrm{SO}_{4}\right)$ and evaporated to give an orange solid. Further purification was carried out by flash chromatography (DCM : $\mathrm{MeOH}=100: 1)$ generating 1o in 26\% yield as a yellow solid. ${ }^{\mathbf{1}} \mathbf{H}$ NMR (400 $\left.\mathbf{~ M H z}, \mathbf{C D C l}_{3}\right) \delta 8.48(\mathrm{~d}, J=8.4 \mathrm{~Hz}, 2 \mathrm{H}), 7.51$ (d, $J=7.7 \mathrm{~Hz}, 2 \mathrm{H}), 7.22$ (t, $J=7.6 \mathrm{~Hz}, 2 \mathrm{H}), 7.14$ (t, $J=7.4 \mathrm{~Hz}, 2 \mathrm{H}), 6.83$ (s, 2H), 4.20 (s, 2H), $1.21(\mathrm{~d}, J=14.7 \mathrm{~Hz}, 36 \mathrm{H}) .{ }^{13} \mathbf{C}$ NMR (101 MHz, CDCl $\mathbf{~}_{3} \delta 142.14$, $128.72(\mathrm{~d}, J=5.4 \mathrm{~Hz}), 124.18(\mathrm{~d}, J=4.7 \mathrm{~Hz}), 123.61,121.14,118.94$ (d, $J=4.8 \mathrm{~Hz})$, 118.49, 116.31, $38.37(\mathrm{~d}, J=69.4 \mathrm{~Hz}), 26.51,21.22 .{ }^{31} \mathbf{P}$ NMR (162 MHz, $\left.\mathbf{C D C l}_{3}\right) \delta$ 62.31. ATR-FTIR (cm $\left.{ }^{-1}\right):$ 3146, 1683, 1400, 1299, 1181, 1135, 1016, 976, 816, 745, 660, 620. HRMS m/z (ESI): calcd for $\mathrm{C}_{33} \mathrm{H}_{49} \mathrm{~N}_{2} \mathrm{O}_{2} \mathrm{P}_{2}(\mathrm{M}+\mathrm{H})^{+}$567.3264, found 567.3265 . 


\section{General Procedure for C6-Selective C-H Arylation of Indoles.}

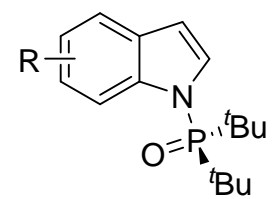

1

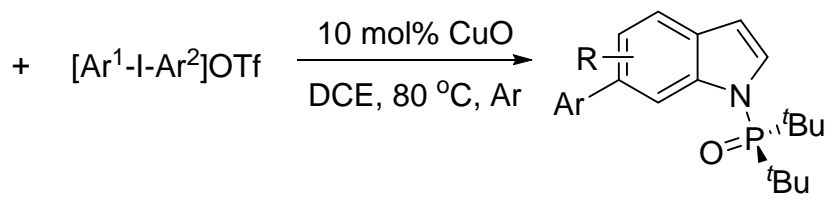

3

To an oven dried $25 \mathrm{~mL}$ Schlenk tube was charged with a stirred bar, indole substrates $1(0.10-0.20 \mathrm{mmol}), \mathrm{DCE}(3-6 \mathrm{~mL})$ and keep stirred for $5 \mathrm{~min}$. The tube was purged with Ar for three times, then diaryliodonium salts $2(0.15-0.30 \mathrm{mmol})$ and $\mathrm{CuO}(0.8-1.6 \mathrm{mg}, 10 \mathrm{~mol} \%)$, were added into the solution of indoles in DCE (3-6 mL). The formed mixture was stirred at $80{ }^{\circ} \mathrm{C}$ under Ar for $12 \mathrm{~h}$ as monitored by TLC. The solution was then cooled to $\mathrm{rt}$, and the solvent was removed under vaccum directly. The crude product was purified by column chromatography on silica gel (petroleum ether / Ethyl acetate) to afford the pure products.

\section{Di-tert-butyl(6-phenyl-1H-indol-1-yl)phosphine oxide (3aa)}<smiles>CCCCP(=O)(n1ccc2ccc(-c3ccccc3)cc21)C(C)(C)C</smiles>

3aa

Following the above procedure, the reaction of $1 \mathbf{a}(28.0 \mathrm{mg}$, $0.10 \mathrm{mmol}), \mathrm{Ph}_{2} \mathrm{IOTf}$ (2a) (64.5 mg, $\left.0.15 \mathrm{mmol}\right), \mathrm{CuO}(0.8$ $\mathrm{mg}, 10 \mathrm{~mol} \%)$, in $\operatorname{DCE}(3 \mathrm{~mL})$ at $80{ }^{\circ} \mathrm{C}$. After $12 \mathrm{~h}$, purification by column chromatography on silica gel yield 3aa (31.1 mg, 88\%) as a white solid. ${ }^{1} \mathbf{H}$ NMR (400 MHz, $\left.\mathbf{C D C l}_{3}\right) \delta 8.87(\mathrm{~s}, 1 \mathrm{H}), 7.83-7.68(\mathrm{~m}, 2 \mathrm{H}), 7.64(\mathrm{~d}, J=8.2 \mathrm{~Hz}, 1 \mathrm{H}), 7.48(\mathrm{dd}, J=8.2$, $1.5 \mathrm{~Hz}, 1 \mathrm{H}), 7.41(\mathrm{t}, J=7.6 \mathrm{~Hz}, 2 \mathrm{H}), 7.33-7.23(\mathrm{~m}, 2 \mathrm{H}), 6.79-6.56(\mathrm{~m}, 1 \mathrm{H}), 1.37$ $(\mathrm{d}, J=14.7 \mathrm{~Hz}, 18 \mathrm{H}) .{ }^{13} \mathbf{C}$ NMR (101 MHz, $\left.\mathbf{C D C l}_{3}\right) \delta 142.35,141.92,136.61$, 128.54, 128.49, 127.47, 126.93 (d, $J=4.7 \mathrm{~Hz}), 126.59,121.09,120.28,114.77$, $106.93(\mathrm{~d}, J=5.2 \mathrm{~Hz}), 38.64(\mathrm{~d}, J=69.4 \mathrm{~Hz}), 26.71 .{ }^{31} \mathbf{P}$ NMR (162 $\left.\mathbf{M H z}, \mathbf{C D C l}_{3}\right) \delta$ 63.10. ATR-FTIR (cm $\left.{ }^{-1}\right):$ 2987, 1469, 1423, 1308, 1236, 1181, 1130, 996, 820, 757, 699, 661, 604. HRMS m/z (ESI): calcd for $\mathrm{C}_{22} \mathrm{H}_{28} \mathrm{NOPNa}^{+}$376.1801, found 376.1802 . 


\section{Di-tert-butyl(4-methyl-6-phenyl-1H-indol-1-yl)phosphine oxide (3ba)}<smiles>Cc1cc(-c2ccccc2)cc2c1ccn2P(=O)(CC(C)(C)C)C(C)(C)C</smiles>

$3 \mathrm{ba}$

Following the above procedure, the reaction of $\mathbf{1 b}(58.3 \mathrm{mg}$, $0.20 \mathrm{mmol}), \mathrm{Ph}_{2} \mathrm{IOTf}$ (2a) (129.1 mg, $\left.0.30 \mathrm{mmol}\right), \mathrm{CuO}$ (1.6 $\mathrm{mg}, 10 \mathrm{~mol} \%)$, in $\operatorname{DCE}(6 \mathrm{~mL})$ at $80{ }^{\circ} \mathrm{C}$. After $12 \mathrm{~h}$, purification by column chromatography on silica gel yield 3ba (59.1 mg, 80\%) as a white solid. ${ }^{1} \mathrm{H}$ NMR (400 MHz,

$\left.\mathbf{C D C l}_{3}\right) \delta 8.70(\mathrm{~s}, 1 \mathrm{H}), 7.72(\mathrm{dd}, J=8.2,1.0 \mathrm{~Hz}, 2 \mathrm{H}), 7.41(\mathrm{t}, J=7.6 \mathrm{~Hz}, 2 \mathrm{H}), 7.28$ (ddd, $J=4.9,3.5,2.7 \mathrm{~Hz}, 3 \mathrm{H}), 6.80-6.60(\mathrm{~m}, 1 \mathrm{H}), 2.61(\mathrm{~s}, 3 \mathrm{H}), 1.37$ (d, $J=14.7 \mathrm{~Hz}$, 18H). ${ }^{13}$ C NMR (101 MHz, $\left.\mathbf{C D C l}_{3}\right) \delta 142.07,136.76,129.50,128.47,128.34(\mathrm{~d}, J=$ $5.4 \mathrm{~Hz}), 127.47,126.49,126.37(\mathrm{~d}, J=4.7 \mathrm{~Hz}), 121.47,112.39,105.36(\mathrm{~d}, J=5.2$ Hz), $38.62(\mathrm{~d}, J=69.3 \mathrm{~Hz}), 26.72,18.93 .{ }^{31} \mathbf{P}$ NMR (162 MHz, $\left.\mathbf{C D C l}_{3}\right) \delta 62.89$. ATR-FTIR (cm $\left.{ }^{-1}\right):$ 3136, 1600, 1400, 1319, 1178, 1137, 814, 761, 736, 700, 662. HRMS m/z (ESI): calcd for $\mathrm{C}_{23} \mathrm{H}_{30} \mathrm{NOPNa}^{+}$390.1957, found 390.1959.

\section{Di-tert-butyl(3-methyl-6-phenyl-1H-indol-1-yl)phosphine oxide (3ca)}<smiles>Cc1cn(P(=O)(CC(C)(C)C)C(C)(C)C)c2cc(-c3ccccc3)ccc12</smiles>

3ca

Following the above procedure, the reaction of 1c $(58.3 \mathrm{mg}$, $0.20 \mathrm{mmol})$, [Mes-I-Ph]OTf (2a') (142 mg, $0.30 \mathrm{mmol}), \mathrm{CuO}$ (1.6 $\mathrm{mg}, 10 \mathrm{~mol} \%)$, in DCE $(6 \mathrm{~mL})$ at $80{ }^{\circ} \mathrm{C}$. After $12 \mathrm{~h}$, purification by column chromatography on silica gel yield 3ca (64.0 mg, 87\%) as a white solid. ${ }^{1} \mathbf{H}$ NMR (400 MHz, $\left.\mathbf{C D C l}_{3}\right) \delta 8.82(\mathrm{~d}, J=1.0 \mathrm{~Hz}, 1 \mathrm{H}), 7.75-7.69(\mathrm{~m}, 2 \mathrm{H}), 7.56$ $(\mathrm{d}, J=8.2 \mathrm{~Hz}, 1 \mathrm{H}), 7.49(\mathrm{dd}, J=8.2,1.6 \mathrm{~Hz}, 1 \mathrm{H}), 7.44-7.37(\mathrm{~m}, 2 \mathrm{H}), 7.32-7.26$ (m, 1H), $7.03-6.97(\mathrm{~m}, 1 \mathrm{H}), 2.35(\mathrm{~d}, J=1.0 \mathrm{~Hz}, 3 \mathrm{H}), 1.36(\mathrm{~d}, J=14.6 \mathrm{~Hz}, 18 \mathrm{H}) .{ }^{13} \mathrm{C}$ NMR (101 MHz, $\left.\mathbf{C D C l}_{3}\right) \delta 142.71,142.00,136.61,129.24$ (d, $\left.J=5.4 \mathrm{~Hz}\right), 128.53$, $127.49,126.54,123.99$ (d, $J=4.6 \mathrm{~Hz}), 120.58,118.39,115.93$ (d, $J=4.8 \mathrm{~Hz}), 114.79$,

$38.55(\mathrm{~d}, J=70.0 \mathrm{~Hz}), 26.73,9.83 .{ }^{31} \mathbf{P}$ NMR (162 $\left.\mathbf{M H z}, \mathbf{C D C l}_{3}\right) \delta 62.39$. ATR-FTIR (cm $\left.{ }^{-1}\right):$ 3132, 1618, 1402, 1137, 814, 661, 600. HRMS m/z (ESI): calcd for $\mathrm{C}_{23} \mathrm{H}_{30} \mathrm{NOPNa}^{+} 390.1957$, found 390.1959 . 


\section{Di-tert-butyl(5-methyl-6-phenyl-1H-indol-1-yl)phosphine oxide (3da)}<smiles>Cc1cc2ccn(P(=O)(CC(C)(C)C)[13C](C)(C)C)c2cc1-c1ccccc1</smiles>

3da

Following the above procedure, the reaction of $\mathbf{1 d}(58.3 \mathrm{mg}$, $0.20 \mathrm{mmol}), \mathrm{Ph}_{2} \mathrm{IOTf}$ (2a) (129.1 mg, $\left.0.30 \mathrm{mmol}\right), \mathrm{CuO}(1.6$ $\mathrm{mg}, 10 \mathrm{~mol} \%)$, in DCE $(6 \mathrm{~mL})$ at $80{ }^{\circ} \mathrm{C}$. After $12 \mathrm{~h}$, purification by column chromatography on silica gel yield 3da (70.0 mg, 95\%) as a white solid. ${ }^{1} \mathbf{H}$ NMR (400 MHz, $\left.\mathbf{C D C l}_{3}\right) \delta 8.42(\mathrm{~s}, 1 \mathrm{H}), 7.47(\mathrm{~s}, 1 \mathrm{H}), 7.41-7.34(\mathrm{~m}, 4 \mathrm{H}), 7.31-7.27(\mathrm{~m}, 1 \mathrm{H}), 7.23$ (dd, $J=3.4,1.4 \mathrm{~Hz}, 1 \mathrm{H}), 6.66-6.62(\mathrm{~m}, 1 \mathrm{H}), 2.35$ (s, 3H), 1.34 (d, $J=14.7 \mathrm{~Hz}, 18 \mathrm{H})$. ${ }^{13}$ C NMR (101 MHz, $\mathbf{C D C l}_{3}$ ) $\delta 142.79,140.26,138.10,129.73,128.81,128.53$ (d, $J$ $=5.4 \mathrm{~Hz}), 127.74,126.75(\mathrm{~d}, J=4.7 \mathrm{~Hz}), 126.23,120.91,117.20,106.50(\mathrm{~d}, J=4.9$ Hz), $38.57(\mathrm{~d}, J=69.8 \mathrm{~Hz}), 26.71,20.83 .{ }^{31} \mathbf{P}$ NMR (162 MHz, $\left.\mathbf{C D C l}_{3}\right) \delta 62.46$. ATR-FTIR (cm $\left.{ }^{-1}\right): 3114,2969,1452,1401,1307,1233,1177,1145,1005,700,664$, 613. HRMS m/z (ESI): calcd for $\mathrm{C}_{23} \mathrm{H}_{30} \mathrm{NOPNa}^{+}$390.1957, found 390.1959 .

\section{Di-tert-butyl(5-methoxy-6-phenyl-1H-indol-1-yl)phosphine oxide (3ea)}<smiles>COc1cc2ccn(P([18CH3])(=O)CC(C)(C)C)c2cc1-c1ccccc1</smiles>

3ea

Following the above procedure, the reaction of 1e $(61.5$ $\mathrm{mg}, 0.20 \mathrm{mmol}), \mathrm{Ph}_{2} \mathrm{IOTf}$ (2a) $(129.1 \mathrm{mg}, 0.30 \mathrm{mmol})$, $\mathrm{CuO}(1.6 \mathrm{mg}, 10 \mathrm{~mol} \%)$, in DCE $(6 \mathrm{~mL})$ at $80{ }^{\circ} \mathrm{C}$. After $12 \mathrm{~h}$, purification by column chromatography on silica gel yield 3ea (55.2 mg, 72\%) as a white solid. ${ }^{1} \mathbf{H}$ NMR (400 MHz, $\left.\mathbf{C D C l}_{3}\right) \delta 8.52(\mathrm{~s}, 1 \mathrm{H}), 7.61(\mathrm{dd}, J=8.2,1.2 \mathrm{~Hz}, 2 \mathrm{H}), 7.37(\mathrm{t}, J=7.5 \mathrm{~Hz}, 2 \mathrm{H})$, $7.28(\mathrm{dt}, J=4.0,1.7 \mathrm{~Hz}, 1 \mathrm{H}), 7.23(\mathrm{dd}, J=3.4,1.5 \mathrm{~Hz}, 1 \mathrm{H}), 7.11(\mathrm{~s}, 1 \mathrm{H}), 6.72-6.59$ $(\mathrm{m}, 1 \mathrm{H}), 3.84(\mathrm{~d}, J=6.2 \mathrm{~Hz}, 3 \mathrm{H}), 1.35(\mathrm{~d}, J=14.7 \mathrm{~Hz}, 18 \mathrm{H}) .{ }^{13} \mathrm{C}$ NMR (101 MHz, $\left.\mathbf{C D C l}_{3}\right) \delta 152.54,139.40,136.51,129.97,128.95(\mathrm{~d}, J=5.4 \mathrm{~Hz}), 128.04,127.75$, $127.04(\mathrm{~d}, J=4.6 \mathrm{~Hz}), 126.43,118.32,106.78(\mathrm{~d}, J=5.2 \mathrm{~Hz}), 101.15,55.78,38.58(\mathrm{~d}$, $J=69.5 \mathrm{~Hz}), 26.71 .{ }^{31} \mathbf{P}$ NMR (162 MHz, $\left.\mathbf{C D C l}_{3}\right) \delta$ 62.62. ATR-FTIR (cm $\left.{ }^{-1}\right): 3132$, 1461, 1401, 1205, 1149, 1130, 1031, 815, 785, 699, 663, 641, 621. HRMS m/z (ESI): calcd for $\mathrm{C}_{23} \mathrm{H}_{30} \mathrm{NO}_{2} \mathrm{PNa}^{+}$406.1906, found 406.1907. 


\section{Di-tert-butyl(4-fluoro-6-phenyl-1H-indol-1-yl)phosphine oxide (3fa)}<smiles>CC(C)(C)CP(=O)(n1ccc2c(F)cc(-c3ccccc3)cc21)C(C)(C)C</smiles>

3fa

Following the above procedure, the reaction of $\mathbf{1 f}(59.1 \mathrm{mg}$, $0.20 \mathrm{mmol}), \mathrm{Ph}_{2} \mathrm{IOTf}$ (2a) (129.1 mg, $\left.0.30 \mathrm{mmol}\right), \mathrm{CuO}$ (1.6 $\mathrm{mg}, 10 \mathrm{~mol} \%)$, in $\operatorname{DCE}(6 \mathrm{~mL})$ at $80{ }^{\circ} \mathrm{C}$. After $12 \mathrm{~h}$, purification by column chromatography on silica gel yield 3fa (63.1 mg, 85\%) as a white solid. ${ }^{1} \mathrm{H}$ NMR (400 MHz, $\left.\mathbf{C D C l}_{3}\right) \delta 8.65(\mathrm{~s}, 1 \mathrm{H}), 7.68(\mathrm{dd}, J=8.1,1.0 \mathrm{~Hz}, 2 \mathrm{H}), 7.45-$ $7.37(\mathrm{~m}, 2 \mathrm{H}), 7.31(\mathrm{t}, J=7.3 \mathrm{~Hz}, 1 \mathrm{H}), 7.23(\mathrm{dd}, J=3.5,1.2 \mathrm{~Hz}, 1 \mathrm{H}), 7.17$ (dd, $J=$ 11.2, 1.2 Hz, 1H), $6.87-6.71(\mathrm{~m}, 1 \mathrm{H}), 1.37(\mathrm{~d}, J=14.8 \mathrm{~Hz}, 18 \mathrm{H}) .{ }^{13} \mathbf{C}$ NMR (101 MHz, $\left.\mathbf{C D C l}_{3}\right) \delta 155.77(\mathrm{~d}, J=246.4 \mathrm{~Hz}), 144.29$ (d, $\left.J=10.2 \mathrm{~Hz}\right), 140.90(\mathrm{~d}, J=2.2$ $\mathrm{Hz}), 137.63(\mathrm{~d}, J=7.2 \mathrm{~Hz}), 128.65,127.22$ (d, $J=33.9 \mathrm{~Hz}), 126.75,117.62$ (d, J = 23.3 Hz), 110.71, $105.91(\mathrm{~d}, J=19.2 \mathrm{~Hz}), 102.78,38.67(\mathrm{~d}, J=67.1 \mathrm{~Hz}), 26.67 .{ }^{31} \mathbf{P}$ NMR (162 MHz, CDCl $)_{3} \delta$ 63.90. ${ }^{19}$ F NMR (376 $\mathbf{M H z}, \mathbf{C D C l}_{3}$ ) $\delta-122.76$. ATR-FTIR (cm $\left.{ }^{-1}\right): 3135,1626,1511,1401,1326,1179,1132,1037,763,664,619$. HRMS m/z (ESI): calcd for $\mathrm{C}_{22} \mathrm{H}_{27} \mathrm{FNOPNa}^{+}$394.1707, found 394.1707.

\section{Di-tert-butyl(4-chloro-6-phenyl-1H-indol-1-yl)phosphine oxide (3ga)}<smiles>CC(C)(C)CP(=O)(n1ccc2c(Cl)cc(-c3ccccc3)cc21)C(C)(C)C</smiles>
$3 g a$

Following the above procedure, the reaction of $\mathbf{1 g}(62.4$ $\mathrm{mg}, 0.20 \mathrm{mmol}), \mathrm{Ph}_{2} \mathrm{IOTf}$ (2a) (129.1 mg, $0.30 \mathrm{mmol}$ ), $\mathrm{CuO}(1.6 \mathrm{mg}, 10 \mathrm{~mol} \%)$, in DCE $(6 \mathrm{~mL})$ at $80{ }^{\circ} \mathrm{C}$. After $12 \mathrm{~h}$, purification by column chromatography on silica gel yield 3ga (63.3 mg, 82\%) as a white solid. ${ }^{\mathbf{1}} \mathbf{H}$ NMR (400 MHz, $\left.\mathbf{C D C l}_{3}\right) \delta 8.77(\mathrm{~s}, 1 \mathrm{H}), 7.73-7.64(\mathrm{~m}, 2 \mathrm{H}), 7.49(\mathrm{~d}$, $J=1.3 \mathrm{~Hz}, 1 \mathrm{H}), 7.41(\mathrm{t}, J=7.6 \mathrm{~Hz}, 2 \mathrm{H}), 7.35-7.27(\mathrm{~m}, 2 \mathrm{H}), 6.86-6.77(\mathrm{~m}, 1 \mathrm{H})$, $1.36(\mathrm{~d}, J=14.8 \mathrm{~Hz}, 18 \mathrm{H}) .{ }^{13} \mathbf{C}$ NMR (101 MHz, $\left.\mathbf{C D C l}_{3}\right) \delta 142.77,140.73,137.72$, 128.67, 127.44, 127.41, 127.25 (d, $J=5.1 \mathrm{~Hz}), 127.09$ 125.54, 120.79, 113.38, 105.36 (d, $J=4.8 \mathrm{~Hz}), 38.67$ (d, $J=68.64 \mathrm{~Hz}), 26.64 .{ }^{31} \mathbf{P}$ NMR (162 MHz, $\left.\mathbf{C D C l}_{3}\right) \delta 64.00$. ATR-FTIR (cm $\left.{ }^{-1}\right):$ 3148, 2964, 1557, 1510, 1464, 1400, 1313, 1235, 1173, 1136, 1006, 761, 698, 663, 623. HRMS m/z (ESI): calcd for $\mathrm{C}_{22} \mathrm{H}_{27} \mathrm{ClNOPNa}^{+}$410.1411, found 410.1413 . 


\section{Di-tert-butyl(3-chloro-6-phenyl-1H-indol-1-yl)phosphine oxide (3ha)}<smiles>CCCCP(=O)(n1cc(Cl)c2ccc(-c3ccccc3)cc21)C(C)(C)C</smiles>

3ha

Following the above procedure, the reaction of $\mathbf{1 h}(62.4$ mg, $0.20 \mathrm{mmol}), \mathrm{Ph}_{2} \mathrm{IOTf}$ (2a) (129.1 mg, $\left.0.30 \mathrm{mmol}\right)$, $\mathrm{CuO}(1.6 \mathrm{mg}, 10 \mathrm{~mol} \%)$, in DCE $(6 \mathrm{~mL})$ at $80{ }^{\circ} \mathrm{C}$. After $12 \mathrm{~h}$, purification by column chromatography on silica gel yield 3ha (57.5 mg, 74\%) as a white solid. ${ }^{\mathbf{1}} \mathbf{H}$ NMR (400 MHz, $\left.\mathbf{C D C l}_{3}\right) \delta 8.85(\mathrm{~d}, J=1.0 \mathrm{~Hz}, 1 \mathrm{H}), 7.73-7.67(\mathrm{~m}$, 2H), $7.64(\mathrm{~d}, J=8.3 \mathrm{~Hz}, 1 \mathrm{H}), 7.55(\mathrm{dd}, J=8.3,1.5 \mathrm{~Hz}, 1 \mathrm{H}), 7.42$ (dd, $J=10.4,4.8$ $\mathrm{Hz}, 2 \mathrm{H}), 7.35-7.28(\mathrm{~m}, 1 \mathrm{H}), 7.23(\mathrm{~d}, J=1.5 \mathrm{~Hz}, 1 \mathrm{H}), 1.37$ (d, $J=14.9 \mathrm{~Hz}, 18 \mathrm{H})$.

${ }^{13}$ C NMR (101 MHz, $\left.\mathbf{C D C l}_{3}\right) \delta 141.43(\mathrm{~d}, J=2.6 \mathrm{~Hz}), 137.94,128.63,127.53$, 126.94, 125.97 (d, $J=4.4 \mathrm{~Hz}), 122.94$ (d, $J=4.8 \mathrm{~Hz}), 121.73,118.00,115.00,111.29$ $(\mathrm{d}, J=5.2 \mathrm{~Hz}), 38.68(\mathrm{~d}, J=68.4 \mathrm{~Hz}), 26.66 .{ }^{31} \mathbf{P}$ NMR (162 MHz, $\left.\mathbf{C D C l}_{3}\right) \delta 64.55$. ATR-FTIR (cm $\left.{ }^{-1}\right): 3125,1468,1401,1173,1133,1044,951,814,762,664,610$. HRMS m/z (ESI): calcd for $\mathrm{C}_{22} \mathrm{H}_{27} \mathrm{ClNOPNa}^{+}$410.1411, found 410.1411.

\section{Di-tert-butyl(2,3-dichloro-6-phenyl-1H-indol-1-yl)phosphine oxide (3ia)}<smiles>CC(C)(C)P(=O)([18O])n1c(Cl)c(Cl)c2ccc(-c3ccccc3)cc21</smiles>

3ia Following the above procedure, the reaction of $\mathbf{1 i}(69.2$ mg, $0.20 \mathrm{mmol}$ ), $\mathrm{Ph}_{2}$ IOTf (2a) (129.1 mg, $0.30 \mathrm{mmol}$ ), $\mathrm{CuO}(1.6 \mathrm{mg}, 10 \mathrm{~mol} \%)$, in DCE $(6 \mathrm{~mL})$ at $80{ }^{\circ} \mathrm{C}$. After $12 \mathrm{~h}$, purification by column chromatography on silica gel yield 3ia (75.0 mg, 89\%) as a yellow solid. ${ }^{1}$ H NMR (400 MHz, $\left.\mathbf{C D C l}_{3}\right) \delta 9.12(\mathrm{~s}, 1 \mathrm{H}), 7.69(\mathrm{dd}, J=8.2,1.0 \mathrm{~Hz}$, 2H), $7.55(\mathrm{~s}, 2 \mathrm{H}), 7.46-7.37(\mathrm{~m}, 2 \mathrm{H}), 7.31(\mathrm{ddd}, J=7.3,6.2,1.1 \mathrm{~Hz}, 1 \mathrm{H}), 1.45(\mathrm{~d}, J$ $=15.6 \mathrm{~Hz}, 18 \mathrm{H}) .{ }^{13} \mathbf{C}$ NMR (101 MHz, $\left.\mathbf{C D C l}_{3}\right) \delta 141.46,141.17,137.84,128.64$, 127.50, 126.93, 126.06 (d, $J=3.7 \mathrm{~Hz}), 122.12,120.25$ (d, $J=2.3 \mathrm{~Hz}), 117.33,116.57$, $111.06(\mathrm{~d}, J=2.7 \mathrm{~Hz}), 40.74(\mathrm{~d}, J=63.8 \mathrm{~Hz}), 27.16 .{ }^{31} \mathbf{P}$ NMR (162 MHz, $\left.\mathbf{C D C l}_{3}\right) \delta$ 75.45. ATR-FTIR (cm $\left.{ }^{-1}\right): 3123,1684,1401,1206,1059,950,814,762,660,613$. HRMS m/z (ESI): calcd for $\mathrm{C}_{22} \mathrm{H}_{26} \mathrm{Cl}_{2} \mathrm{NOPNa}^{+} 444.1021$, found 444.1020 . 
(4-Bromo-6-phenyl-1H-indol-1-yl)di-tert-butylphosphine oxide (3ja)<smiles>CC(C)(C)CP(=O)(n1ccc2c(Br)cc(-c3ccccc3)cc21)C(C)(C)C</smiles>

$3 \mathbf{j a}$

Following the above procedure, the reaction of $\mathbf{1 j}(71.2 \mathrm{mg}$, $0.20 \mathrm{mmol}), \mathrm{Ph}_{2} \mathrm{IOTf}(2 \mathrm{a})(129.1 \mathrm{mg}, 0.30 \mathrm{mmol}), \mathrm{CuO}(1.6$ $\mathrm{mg}, 10 \mathrm{~mol} \%)$, in $\operatorname{DCE}(6 \mathrm{~mL})$ at $80{ }^{\circ} \mathrm{C}$. After $12 \mathrm{~h}$, purification by column chromatography on silica gel yield 3ja (66.6 mg, 77\%) as a white solid. ${ }^{1} \mathbf{H}$ NMR (400 MHz, $\left.\mathbf{C D C l}_{3}\right) \delta 8.83(\mathrm{~s}, 1 \mathrm{H}), 7.67(\mathrm{~d}, J=8.1 \mathrm{~Hz}, 3 \mathrm{H}), 7.41(\mathrm{t}, J=$ $7.6 \mathrm{~Hz}, 2 \mathrm{H}), 7.35-7.28(\mathrm{~m}, 2 \mathrm{H}), 6.77$ (s, 1H), 1.36 (d, $J=14.8 \mathrm{~Hz}, 18 \mathrm{H}) .{ }^{13} \mathbf{C}$ NMR (101 MHz, $\left.\mathbf{C D C l}_{3}\right) \delta 142.31,140.60,138.00,129.08$ (d, $\left.J=4.9 \mathrm{~Hz}\right), 128.66,127.42$, 127.38, 127.10, 123.93, 114.08, 113.91, 107.04 (d, $J=4.5 \mathrm{~Hz}), 38.67(\mathrm{~d}, J=68.6 \mathrm{~Hz})$, 26.63. ${ }^{31}$ P NMR (162 MHz, $\left.\mathbf{C D C l}_{3}\right) \delta$ 64.14. ATR-FTIR (cm $\left.{ }^{-1}\right): 3131,1505,1404$, 1305, 1169, 1139, 999, 815, 758, 661, 619. HRMS m/z (ESI): calcd for $\mathrm{C}_{22} \mathrm{H}_{27} \mathrm{BrNOPNa}^{+}$454.0906, found 454.0907.

\section{Di-tert-butyl(3-iodo-6-phenyl-1H-indol-1-yl)phosphine oxide (3ka)}<smiles>CCCP(=O)(n1cc(I)c2ccc(-c3ccccc3)cc21)C(C)(C)C</smiles>

$3 k a$

Following the above procedure, the reaction of $1 \mathbf{k}(80.6 \mathrm{mg}$, $0.20 \mathrm{mmol}), \mathrm{Ph}_{2} \operatorname{IOTf}$ (2a) (129.1 mg, $\left.0.30 \mathrm{mmol}\right), \mathrm{CuO}(1.6$ $\mathrm{mg}, 10 \mathrm{~mol} \%)$, in DCE $(6 \mathrm{~mL})$ at $80{ }^{\circ} \mathrm{C}$. After $12 \mathrm{~h}$, purification by column chromatography on silica gel yield 3ka (32.2 mg, 34\%) as a white solid. ${ }^{1} \mathbf{H}$ NMR (400 MHz, $\left.\mathbf{C D C l}_{3}\right) \delta 8.84(\mathrm{~d}, J=0.8 \mathrm{~Hz}, 1 \mathrm{H}), 7.70(\mathrm{~d}, J=7.3 \mathrm{~Hz}, 2 \mathrm{H})$, $7.56(\mathrm{dd}, J=8.3,1.4 \mathrm{~Hz}, 1 \mathrm{H}), 7.48-7.38(\mathrm{~m}, 3 \mathrm{H}), 7.35-7.28(\mathrm{~m}, 2 \mathrm{H}), 1.37$ (d, $J=$ $14.9 \mathrm{~Hz}, 18 \mathrm{H}) .{ }^{13} \mathbf{C}$ NMR (101 MHz, $\left.\mathbf{C D C l}_{3}\right) \delta 141.83,141.45,137.88,130.45(\mathrm{~d}, J$ $=4.2 \mathrm{~Hz}), 130.26(\mathrm{~d}, J=4.8 \mathrm{~Hz}), 128.63,127.56,126.91,121.94,120.87,114.77$, 64.04, $38.71(\mathrm{~d}, J=68.42 \mathrm{~Hz}), 26.68 .{ }^{31} \mathbf{P}$ NMR (162 $\left.\mathbf{M H z}, \mathbf{C D C l}_{3}\right) \delta 64.46$. ATR-FTIR (cm $\left.{ }^{-1}\right): 3125,1683,1465,1401,1132,1021,930,812,763,701,662$, 605. HRMS m/z (ESI): calcd for $\mathrm{C}_{22} \mathrm{H}_{27} \mathrm{INOPNa}^{+}$502.0767, found 502.0767. 
Methyl 1-(di-tert-butylphosphoryl)-6-phenyl-1H-indole-3-carboxylate (3la)<smiles>COC(=O)c1cn(P(=O)(CC(C)(C)C)C(C)(C)C)c2cc(-c3ccccc3)ccc12</smiles>

3la $0.20 \mathrm{mmol}), \mathrm{Ph}_{2} \mathrm{IOTf}$ (2a) (129.1 mg, $\left.0.30 \mathrm{mmol}\right), \mathrm{CuO}$ $(1.6 \mathrm{mg}, 10 \mathrm{~mol} \%)$, in DCE $(6 \mathrm{~mL})$ at $80{ }^{\circ} \mathrm{C}$. After $12 \mathrm{~h}$, purification by column chromatography on silica gel yield 3la $(65.1 \mathrm{mg}, 79 \%)$ as a white solid. ${ }^{\mathbf{1}} \mathbf{H}$ NMR (400 MHz, $\left.\mathbf{C D C l}_{3}\right) \delta 8.88(\mathrm{~d}, J=1.1 \mathrm{~Hz}, 1 \mathrm{H}), 8.19(\mathrm{~d}, J=8.3 \mathrm{~Hz}, 1 \mathrm{H})$, $7.94(\mathrm{~d}, J=2.0 \mathrm{~Hz}, 1 \mathrm{H}), 7.74-7.68(\mathrm{~m}, 2 \mathrm{H}), 7.61(\mathrm{dd}, J=8.3,1.6 \mathrm{~Hz}, 1 \mathrm{H}), 7.44-$ 7.35 (m, 2H), $7.34-7.28(\mathrm{~m}, 1 \mathrm{H}), 3.96$ (s, 3H), 1.38 (d, $J=15.0 \mathrm{~Hz}, 18 \mathrm{H}) .{ }^{13} \mathrm{C}$ NMR (101 MHz, $\left.\mathbf{C D C l}_{3}\right) \delta 164.85,142.89,141.34,137.60,132.93$ (d, $\left.J=4.7 \mathrm{~Hz}\right), 128.60$, 127.43, 126.89, 125.69 (d, $J=4.3 \mathrm{~Hz}), 122.66,121.04,114.88,112.40$ (d, $J=4.6 \mathrm{~Hz})$, 51.40, $38.69(\mathrm{~d}, J=67.5 \mathrm{~Hz}), 26.61 .{ }^{31} \mathbf{P}$ NMR (162 $\left.\mathbf{M H z}, \mathbf{C D C l}_{3}\right) \delta 65.86$. ATR-FTIR (cm $\left.{ }^{-1}\right): 3156,1715,1545,1476,1440,1400,1262,1200,1141,1070$, 987, 933, 827, 777, 662, 612. HRMS m/z (ESI): calcd for $\mathrm{C}_{24} \mathrm{H}_{30} \mathrm{NO}_{3} \mathrm{PNa}^{+} 434.1856$, found 434.1855 .

\section{(E)-Di-tert-butyl(6-phenyl-4-styryl-1H-indol-1-yl)phosphine oxide (3ma)}<smiles></smiles>

$3 m a$

Following the above procedure, the reaction of $1 \mathbf{m}(75.9$ $\mathrm{mg}, 0.20 \mathrm{mmol}$ ), $\mathrm{Ph}_{2} \mathrm{IOTf}$ (2a) (129.1 mg, $\left.0.30 \mathrm{mmol}\right)$, $\mathrm{CuO}(1.6 \mathrm{mg}, 10 \mathrm{~mol} \%)$, in DCE $(6 \mathrm{~mL})$ at $80{ }^{\circ} \mathrm{C}$. After 12 h, purification by column chromatography on silica gel yield 3ma (62.0 mg, 68\%) as a yellow solid. ${ }^{1} \mathbf{H}$ NMR (400 MHz, $\left.\mathbf{C D C l}_{3}\right) \delta 8.82(\mathrm{~s}, 1 \mathrm{H}), 7.78-7.73(\mathrm{~m}, 3 \mathrm{H})$, $7.62-7.50(\mathrm{~m}, 3 \mathrm{H}), 7.46-7.37(\mathrm{~m}, 4 \mathrm{H}), 7.35-7.28(\mathrm{~m}$, 4H), $7.02-6.96(\mathrm{~m}, 1 \mathrm{H}), 1.39$ (d, $J=14.7 \mathrm{~Hz}, 18 \mathrm{H}) .{ }^{13} \mathbf{C} \mathbf{N M R}\left(101 \mathbf{M H z}, \mathbf{C D C l}_{3}\right) \delta$ $142.79,141.88,137.74,137.06,129.76,129.38,128.70,128.59,127.55,127.17$ (d, J $=4.6 \mathrm{~Hz}), 126.77,126.58,126.53,118.27,114.35,105.25$ (d, $J=4.9 \mathrm{~Hz}), 38.71$ (d, $J$ $=69.15 \mathrm{~Hz}), 26.74 .{ }^{31} \mathbf{P}$ NMR (162 $\left.\mathbf{~ M H z}, \mathbf{C D C l}_{3}\right) \delta$ 63.32. ATR-FTIR (cm $\left.{ }^{-\mathbf{1}}\right):$ 3133, 1685, 1507, 1401, 1310, 1177, 1136, 957, 813, 764, 662, 631. HRMS m/z (ESI): calcd for $\mathrm{C}_{30} \mathrm{H}_{34} \mathrm{NOPNa}^{+} 478.2270$, found 478.2271 . 
Methyl 4-(1-(di-tert-butylphosphoryl)-6-phenyl-1H-indol-3-yl)butanoate (3na)

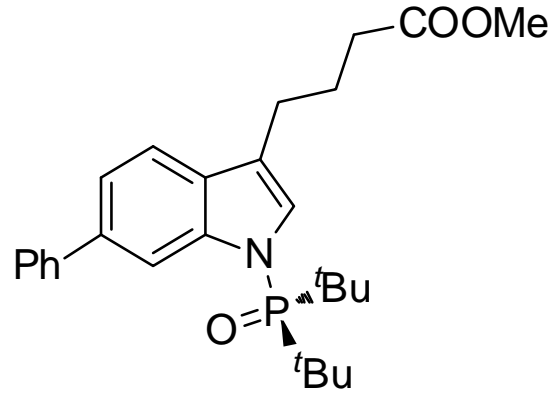

3na

(75.5 mg, $0.20 \mathrm{mmol}), \mathrm{Ph}_{2} \mathrm{IOTf}$ (2a) (129.1 mg, $0.30 \mathrm{mmol}), \mathrm{CuO}(1.6 \mathrm{mg}, 10 \mathrm{~mol} \%)$, in DCE (6 $\mathrm{mL}$ ) at $80{ }^{\circ} \mathrm{C}$. After $12 \mathrm{~h}$, purification by column chromatography on silica gel yield 3na $(84.4 \mathrm{mg}$, 93\%) as a yellow solid. ${ }^{1}$ H NMR (400 MHz, $\left.\mathbf{C D C l}_{3}\right) \delta 8.84(\mathrm{~d}, J=1.1 \mathrm{~Hz}, 1 \mathrm{H}), 7.71(\mathrm{dd}, J=$ $8.2,1.1 \mathrm{~Hz}, 2 \mathrm{H}), 7.59(\mathrm{~d}, J=8.2 \mathrm{~Hz}, 1 \mathrm{H}), 7.48(\mathrm{dd}, J=8.2,1.6 \mathrm{~Hz}, 1 \mathrm{H}), 7.41(\mathrm{t}, J=$ $7.6 \mathrm{~Hz}, 2 \mathrm{H}), 7.29$ (ddd, $J=6.7,2.2,1.1 \mathrm{~Hz}, 1 \mathrm{H}), 7.03(\mathrm{~d}, J=0.7 \mathrm{~Hz}, 1 \mathrm{H}), 3.68$ (s, 3H), $2.82(\mathrm{t}, J=7.4 \mathrm{~Hz}, 2 \mathrm{H}), 2.41(\mathrm{t}, J=7.3 \mathrm{~Hz}, 2 \mathrm{H}), 2.08(\mathrm{p}, J=7.4 \mathrm{~Hz}, 2 \mathrm{H}), 1.36$ $(\mathrm{d}, J=14.7 \mathrm{~Hz}, 18 \mathrm{H}) .{ }^{13} \mathbf{C}$ NMR (101 MHz, $\left.\mathbf{C D C l}_{3}\right) \delta 173.87,142.84,141.87$, 136.69, 128.51, 128.22 (d, $J=5.4 \mathrm{~Hz}), 127.44,126.56,123.95$ (d, $J=4.7 \mathrm{~Hz}), 120.63$, $119.52(\mathrm{~d}, J=4.6 \mathrm{~Hz}), 118.45,114.87,51.51,38.54(\mathrm{~d}, J=69.4 \mathrm{~Hz}), 33.42,26.69$, 24.80, 24.32. ${ }^{31} \mathbf{P}$ NMR (162 MHz, $\left.\mathbf{C D C l}_{3}\right) \delta$ 62.65. ATR-FTIR (cm $\left.{ }^{\mathbf{- 1}}\right): 3125,1736$, 1401, 1313, 1172, 1133, 974, 892, 815, 750, 663, 611. HRMS m/z (ESI): calcd for $\mathrm{C}_{27} \mathrm{H}_{36} \mathrm{NO}_{3} \mathrm{PNa}^{+}$476.2325, found 476.2327.

(3,3'-Methylenebis(6-phenyl-1H-indole-3,1-diyl))bis(di-tert-butylphosphine oxide) (3оa)<smiles>CC(C)(C)CP(=O)(n1cc(Cc2cn([P@@](=O)(CC(C)(C)C)C(C)(C)C)c3cc(-c4ccccc4)ccc23)c2ccc(-c3ccccc3)cc21)C(C)(C)C</smiles>

Following the above procedure, the reaction of $\mathbf{1 0}$ (56.7 mg, $0.10 \mathrm{mmol}), \mathrm{Ph}_{2} \mathrm{IOTf}$ (2a) (129.1 mg, $0.30 \mathrm{mmol}), \mathrm{CuO}(1.6 \mathrm{mg}, 20 \mathrm{~mol} \%)$, in DCE (6 $\mathrm{mL}$ ) at $80{ }^{\circ} \mathrm{C}$. After $12 \mathrm{~h}$, purification by column chromatography on silica gel yield 3oa $(59.3 \mathrm{mg}$, 82\%) as a white solid. ${ }^{1} \mathbf{H}$ NMR (400 MHz, $\left.\mathbf{C D C l}_{3}\right) \delta 8.84(\mathrm{~d}, J=1.0 \mathrm{~Hz}, 2 \mathrm{H}), 7.76-7.68(\mathrm{~m}$, 4H), $7.59(\mathrm{~d}, J=8.2 \mathrm{~Hz}, 2 \mathrm{H}), 7.48(\mathrm{dd}, J=8.2,1.5 \mathrm{~Hz}, 2 \mathrm{H}), 7.42(\mathrm{t}, J=7.6 \mathrm{~Hz}, 4 \mathrm{H})$, $7.31(\mathrm{t}, J=7.3 \mathrm{~Hz}, 2 \mathrm{H}), 6.90(\mathrm{~s}, 2 \mathrm{H}), 4.25(\mathrm{~s}, 2 \mathrm{H}), 1.26(\mathrm{~d}, J=14.7 \mathrm{~Hz}, 36 \mathrm{H}) .{ }^{13} \mathrm{C}$ NMR (101 MHz, $\left.\mathbf{C D C l}_{3}\right) \delta 142.92,141.81,136.85,128.57,128.11(\mathrm{~d}, J=5.3 \mathrm{~Hz})$, 
127.43, 126.68, 124.84 (d, $J=5.0 \mathrm{~Hz}), 120.72,118.87$ (d, $J=5.0 \mathrm{~Hz}), 118.77,114.88$, $38.50(\mathrm{~d}, J=69.4 \mathrm{~Hz}), 26.60,21.38 .{ }^{31} \mathbf{P}$ NMR (162 $\left.\mathbf{M H z}, \mathbf{C D C l}_{3}\right) \delta 62.70$. ATR-FTIR (cm $\left.{ }^{-1}\right):$ 3130, 1608, 1471, 1400, 1199, 1131, 978, 813, 749, 699, 661, 629. HRMS m/z (ESI): calcd for $\mathrm{C}_{45} \mathrm{H}_{56} \mathrm{~N}_{2} \mathrm{O}_{2} \mathrm{P}_{2} \mathrm{Na}^{+} 741.3709$, found 741.3713.

\section{Di-tert-butyl(6-(p-tolyl)-1H-indol-1-yl)phosphine oxide (3ab)}<smiles>Cc1ccc(-c2ccc3ccn(P(=O)(CC(C)C)C(C)(C)C)c3c2)cc1</smiles>

Following the above procedure, the reaction of 1a (55.5 mg, $0.20 \mathrm{mmol})$, mesityl(p-tolyl)iodonium triflate (2b) (145.9 mg, $0.30 \mathrm{mmol}), \mathrm{CuO}(1.6 \mathrm{mg}$, $10 \mathrm{~mol} \%)$, in DCE $(6 \mathrm{~mL})$ at $80{ }^{\circ} \mathrm{C}$. After $12 \mathrm{~h}$, purification by column chromatography on silica gel yield 3ab (64.8 mg, 88\%) as a white solid. ${ }^{1} \mathbf{H}$ NMR (400 MHz, $\left.\mathbf{C D C l}_{3}\right) \delta 8.84$ (s, 1H), $7.62(\mathrm{~d}, J=8.1 \mathrm{~Hz}, 3 \mathrm{H}), 7.47(\mathrm{dd}, J=8.2,1.6 \mathrm{~Hz}, 1 \mathrm{H}), 7.25(\mathrm{dd}, J=3.5,1.4 \mathrm{~Hz}$, 1H), $7.22(\mathrm{~d}, J=7.9 \mathrm{~Hz}, 2 \mathrm{H}), 6.75-6.65(\mathrm{~m}, 1 \mathrm{H}), 2.38(\mathrm{~s}, 3 \mathrm{H}), 1.36(\mathrm{~d}, J=14.7 \mathrm{~Hz}$, 18H). ${ }^{13}$ C NMR (101 MHz, $\left.\mathbf{C D C l}_{3}\right) \delta$ 142.36, 139.00, 136.52, 136.26, 129.28, $128.31(\mathrm{~d}, J=5.4 \mathrm{~Hz}), 127.28,126.80(\mathrm{~d}, J=5.1 \mathrm{~Hz}), 120.96,120.21,114.47$, $106.92(\mathrm{~d}, J=5.0 \mathrm{~Hz}), 38.63(\mathrm{~d}, J=69.4 \mathrm{~Hz}), 26.72,21.06 .{ }^{31} \mathbf{P}$ NMR (162 MHz, $\left.\mathbf{C D C l}_{3}\right) \delta$ 62.99. ATR-FTIR (cm $\left.{ }^{-1}\right): 3134,1515,1469,1401,1305,1174,1137,996$, 804, 664, 630. HRMS m/z (ESI): calcd for $\mathrm{C}_{23} \mathrm{H}_{30} \mathrm{NOPNa}^{+}$390.1957, found 390.1958 .

\section{Di-tert-butyl(6-(m-tolyl)-1H-indol-1-yl)phosphine oxide (3ac)}<smiles>Cc1cccc(-c2ccc3ccn(P(=O)(CC(C)(C)C)CC(C)(C)C)c3c2)c1</smiles>

Following the above procedure, the reaction of 1a $(55.5$ $\mathrm{mg}, 0.20 \mathrm{mmol})$, mesityl(m-tolyl)iodonium triflate (2c) (145.9 mg, $0.30 \mathrm{mmol}), \mathrm{CuO}(1.6 \mathrm{mg}, 10 \mathrm{~mol} \%)$, in DCE $(6 \mathrm{~mL})$ at $80{ }^{\circ} \mathrm{C}$. After $12 \mathrm{~h}$, purification by column chromatography on silica gel yield 3ac $(65.4 \mathrm{mg}, 89 \%)$ as a yellow oil. ${ }^{1}$ H NMR (400 MHz, $\left.\mathbf{C D C l}_{3}\right) \delta 8.84(\mathrm{~s}, 1 \mathrm{H}), 7.63(\mathrm{~d}, J=8.2 \mathrm{~Hz}, 1 \mathrm{H}), 7.56-7.50(\mathrm{~m}, 2 \mathrm{H}), 7.48(\mathrm{dd}$, $J=8.2,1.4 \mathrm{~Hz}, 1 \mathrm{H}), 7.31(\mathrm{t}, J=7.5 \mathrm{~Hz}, 1 \mathrm{H}), 7.27(\mathrm{t}, J=1.7 \mathrm{~Hz}, 1 \mathrm{H}), 7.13(\mathrm{~d}, J=7.4$ $\mathrm{Hz}, 1 \mathrm{H}), 6.74-6.65(\mathrm{~m}, 1 \mathrm{H}), 2.43(\mathrm{~s}, 3 \mathrm{H}), 1.38$ (d, $J=14.7 \mathrm{~Hz}, 18 \mathrm{H}) .{ }^{13} \mathbf{C}$ NMR (101 
MHz, $\left.\mathbf{C D C l}_{3}\right) \delta 142.30,141.96,138.08,136.78,128.48,128.45,128.15,127.38$, $126.86(\mathrm{~d}, J=5.0 \mathrm{~Hz}), 124.69,121.24,120.21,114.71,106.91(\mathrm{~d}, J=4.7 \mathrm{~Hz}), 38.63$ $(\mathrm{d}, J=69.3 \mathrm{~Hz}), 26.72,21.57 .{ }^{31} \mathbf{P}$ NMR (162 $\left.\mathbf{~ M H z ,} \mathbf{C D C l}_{3}\right) \delta$ 63.06. ATR-FTIR $\left(\mathbf{c m}^{-1}\right)$ : 3144, 2967, 1606, 1476, 1402, 1279, 1180, 1136, 997, 815, 788, 662, 605. HRMS m/z (ESI): calcd for $\mathrm{C}_{23} \mathrm{H}_{30} \mathrm{NOPNa}^{+}$390.1957, found 390.1959.

\section{Di-tert-butyl(6-(o-tolyl)-1H-indol-1-yl)phosphine oxide (3ad)}<smiles>Cc1ccccc1-c1ccc2ccn(P(=O)(CC(C)(C)C)C(C)(C)C)c2c1</smiles>

Following the above procedure, the reaction of 1a $(55.5$ $\mathrm{mg}, 0.20 \mathrm{mmol})$, mesityl(o-tolyl)iodonium triflate (2d) (145.9 mg, $0.30 \mathrm{mmol}), \mathrm{CuO}$ (1.6 mg, $10 \mathrm{~mol} \%$ ), in DCE

$(6 \mathrm{~mL})$ at $80{ }^{\circ} \mathrm{C}$. After $12 \mathrm{~h}$, purification by column chromatography on silica gel yield 3ad (54.1 mg, 73\%) as a white solid. ${ }^{1} \mathbf{H}$ NMR (400 MHz, $\left.\mathbf{C D C l}_{3}\right) \delta 8.55(\mathrm{~s}, 1 \mathrm{H}), 7.61$ (d, J=8.1 Hz, 1H), 7.347 .29 (m, 1H), $7.28-$ $7.26(\mathrm{~m}, 1 \mathrm{H}), 7.25-7.16(\mathrm{~m}, 4 \mathrm{H}), 6.74-6.69(\mathrm{~m}, 1 \mathrm{H}), 2.32$ (s, 3H), 1.36 (d, J = 14.7 $\mathrm{Hz}, 18 \mathrm{H}) .{ }^{13} \mathbf{C}$ NMR (101 MHz, $\left.\mathbf{C D C l}_{3}\right) \delta$ 142.64, 141.58, 137.29, 135.55, 130.32, 130.15, 127.88 (d, J = 5.5 Hz), 126.73 (d, J = 4.8 Hz), 126.68, 125.52, 123.24, 119.45, 116.98, $106.92(\mathrm{~d}, J=4.7 \mathrm{~Hz}), 38.58(\mathrm{~d}, J=69.4 \mathrm{~Hz}), 26.71,20.76 .{ }^{31}$ P NMR (162 MHz, $\left.\mathbf{C D C l}_{3}\right) \delta$ 62.82. ATR-FTIR (cm $\left.{ }^{-1}\right): 3137,1684,1401,1176,1139,995,818$, 756, 664, 605. HRMS m/z (ESI): calcd for $\mathrm{C}_{23} \mathrm{H}_{30} \mathrm{NOPNa}^{+}$390.1957, found 390.1960 .

\section{Di-tert-butyl(6-(4-isobutylphenyl)-1H-indol-1-yl)phosphine oxide (3ae)}<smiles>CC(C)Cc1ccc(-c2ccc3ccn(P(=O)(CC(C)C)CC(C)C)c3c2)cc1</smiles>
Following the above procedure, the reaction

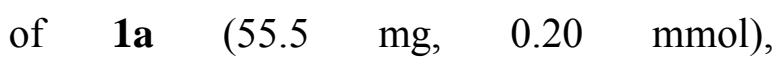
(4-isobutylphenyl)(mesityl)iodonium triflate (2e) $(158.5 \mathrm{mg}, 0.30 \mathrm{mmol}), \mathrm{CuO}(1.6 \mathrm{mg}$, $10 \mathrm{~mol} \%)$, in DCE $(6 \mathrm{~mL})$ at $80^{\circ} \mathrm{C}$. After $12 \mathrm{~h}$, purification by column chromatography on silica gel yield 3ae $(66.1 \mathrm{mg}, 81 \%)$ as a white solid. ${ }^{1} \mathbf{H}$ NMR (400 MHz, $\left.\mathbf{C D C l}_{3}\right) \delta 8.84(\mathrm{~s}, 1 \mathrm{H}), 7.66-7.58(\mathrm{~m}, 3 \mathrm{H}), 7.47(\mathrm{dd}, J=8.2,1.4 \mathrm{~Hz}, 1 \mathrm{H})$, $7.25(\mathrm{dd}, J=3.4,1.2 \mathrm{~Hz}, 1 \mathrm{H}), 7.18(\mathrm{~d}, J=8.0 \mathrm{~Hz}, 2 \mathrm{H}), 6.73-6.65(\mathrm{~m}, 1 \mathrm{H}), 2.50$ (d, $J$ 
$=7.2 \mathrm{~Hz}, 2 \mathrm{H}), 1.97-1.81(\mathrm{~m}, 1 \mathrm{H}), 1.36(\mathrm{~d}, J=14.7 \mathrm{~Hz}, 18 \mathrm{H}), 0.93(\mathrm{~d}, J=6.6 \mathrm{~Hz}$, 6H). ${ }^{13}$ C NMR (101 MHz, $\left.\mathbf{C D C l}_{3}\right) \delta 142.37,140.10,139.25,136.62,129.33,128.27$ (d, $J=5.4 \mathrm{~Hz}), 127.11,126.77$ (d, $J=5.0 \mathrm{~Hz}), 120.96,120.18,114.53,106.92$ (d, $J=$ $4.7 \mathrm{~Hz}), 45.04,38.63(\mathrm{~d}, J=69.3 \mathrm{~Hz}), 30.27,26.71,22.39 .{ }^{31} \mathbf{P}$ NMR (162 MHz, $\left.\mathbf{C D C l}_{3}\right) \delta$ 62.97. ATR-FTIR (cm $\left.{ }^{-1}\right): 3136,2956,1515,1469,1401,1175,1137,995$, 814, 794, 663, 603. HRMS m/z (ESI): calcd for $\mathrm{C}_{26} \mathrm{H}_{36} \mathrm{NOPNa}^{+} 432.2427$, found 432.2428 .

(6-([1,1'-Biphenyl]-4-yl)-1H-indol-1-yl)di-tert-butylphosphine oxide (3af)<smiles></smiles>

3af

Following the above procedure, the reaction of $\mathbf{1 a}$ $(55.5$ $\mathrm{mg}$, 0.20 mmol), [1,1'-biphenyl]-4-yl(mesityl)iodonium triflate (2f) (164.5 mg, $0.30 \mathrm{mmol}), \mathrm{CuO}(1.6 \mathrm{mg}, 10 \mathrm{~mol} \%)$, in

DCE $(6 \mathrm{~mL})$ at $80{ }^{\circ} \mathrm{C}$. After $12 \mathrm{~h}$, purification by column chromatography on silica gel yield 3af (66.4 mg, 77\%) as a yellow solid. ${ }^{1} \mathbf{H}$ NMR (400 MHz, $\left.\mathbf{C D C l}_{3}\right) \delta 8.92$ (s, 1H), $7.84-7.77(\mathrm{~m}, 2 \mathrm{H}), 7.68-7.62(\mathrm{~m}, 5 \mathrm{H}), 7.53(\mathrm{dd}, J=8.2,1.6 \mathrm{~Hz}, 1 \mathrm{H}), 7.49-$ $7.42(\mathrm{~m}, 2 \mathrm{H}), 7.38-7.32(\mathrm{~m}, 1 \mathrm{H}), 7.28(\mathrm{dd}, J=3.5,1.4 \mathrm{~Hz}, 1 \mathrm{H}), 6.75-6.66(\mathrm{~m}, 1 \mathrm{H})$, $1.38(\mathrm{~d}, J=14.7 \mathrm{~Hz}, 18 \mathrm{H}) .{ }^{13} \mathbf{C}$ NMR (101 MHz, $\left.\mathbf{C D C l}_{3}\right) \delta 142.38,140.96,140.87$, 139.42, 136.01, 128.72, 128.61 (d, $J=5.4 \mathrm{~Hz})$, 127.77, 127.31, 127.10, 127.03, 127.00, 120.96, 120.36, 114.65, 106.97 (d, $J=4.7 \mathrm{~Hz}), 38.65$ (d, $J=69.3 \mathrm{~Hz}), 26.72$. ${ }^{31}$ P NMR (162 MHz, CDCl $\left.{ }_{3}\right) \delta$ 63.20. ATR-FTIR (cm $\left.{ }^{-1}\right): 3141,1684,1468,1401$, 1281, 1175, 1134, 995, 814, 762, 662, 603. HRMS m/z (ESI): calcd for $\mathrm{C}_{28} \mathrm{H}_{32} \mathrm{NOPNa}^{+}$452.2114, found 452.2114.

\section{Di-tert-butyl(6-(3,4-dimethoxyphenyl)-1H-indol-1-yl)phosphine oxide (3ag)}<smiles>COc1ccc(-c2ccc3ccn(P(=O)(CC(C)(C)C)CC(C)(C)C)c3c2)cc1OC</smiles>

Following the above procedure, the reaction of $\mathbf{1 a}$ $(55.5$ $\mathrm{mg}$, 0.20 mmol), (3,4-dimethoxyphenyl)(mesityl)iodonium triflate (2g) $(159.7 \mathrm{mg}, 0.30 \mathrm{mmol}), \mathrm{CuO}(1.6 \mathrm{mg}, 10$ $\mathrm{mol} \%)$, in DCE $(6 \mathrm{~mL})$ at $80{ }^{\circ} \mathrm{C}$. After $12 \mathrm{~h}$, purification by column chromatography 
on silica gel yield 3ag (36.0 mg, 44\%) as a yellow solid. ${ }^{1} \mathbf{H}$ NMR (400 MHz, $\mathbf{C D C l}_{3}$ ) $\delta 8.79(\mathrm{~s}, 1 \mathrm{H}), 7.62(\mathrm{~d}, J=7.9 \mathrm{~Hz}, 1 \mathrm{H}), 7.43(\mathrm{~d}, J=7.9 \mathrm{~Hz}, 1 \mathrm{H}), 7.32-7.17(\mathrm{~m}, 3 \mathrm{H})$, 6.92 (d, $J=8.1 \mathrm{~Hz}, 1 \mathrm{H}), 6.70$ (s, 1H), 3.95 (d, $J=20.8 \mathrm{~Hz}, 6 \mathrm{H}), 1.37$ (d, $J=14.6 \mathrm{~Hz}$, 18H). ${ }^{13}$ C NMR (101 MHz, $\left.\mathbf{C D C l}_{3}\right) \delta$ 148.88, 148.09, 142.26, 136.52, 135.14, $128.18(\mathrm{~d}, J=5.3 \mathrm{~Hz}), 126.78(\mathrm{~d}, J=4.7 \mathrm{~Hz}), 121.00,120.19,119.82,114.30,111.31$, 110.79, 106.88 (d, $J=4.8 \mathrm{~Hz}), 55.92$ (d, $J=2.5 \mathrm{~Hz}), 38.58$ (d, $J=69.3 \mathrm{~Hz}), 26.68$. ${ }^{31}$ P NMR (162 MHz, CDCl $)$ ) $\delta$ 63.11. ATR-FTIR (cm $\left.{ }^{-1}\right):$ 3118, 2962, 1606, 1517, 1475, 1402, 1250, 1171, 1136, 1027, 836, 658, 638. HRMS m/z (ESI): calcd for $\mathrm{C}_{24} \mathrm{H}_{32} \mathrm{NO}_{3} \mathrm{PNa}^{+}$436.2012, found 436.2013.

\section{Di-tert-butyl(6-(4-fluorophenyl)-1H-indol-1-yl)phosphine oxide (3ah)}<smiles></smiles>

Following the above procedure, the reaction of 1a $(55.5$ $\mathrm{mg}$, 0.20 $\mathrm{mmol})$, (4-fluorophenyl)(mesityl)iodonium triflate (2h) (147.1 mg, $0.30 \mathrm{mmol}), \mathrm{CuO}(1.6 \mathrm{mg}, 10 \mathrm{~mol} \%)$, in

DCE $(6 \mathrm{~mL})$ at $80{ }^{\circ} \mathrm{C}$. After $12 \mathrm{~h}$, purification by column chromatography on silica gel yield 3ah (57.1 mg, 74\%) as a white solid. ${ }^{1} \mathbf{H}$ NMR (400 $\left.\mathbf{~ M H z , ~} \mathbf{C D C l}_{3}\right) \delta 8.80(\mathrm{~s}$, 1H), $7.68-7.59(\mathrm{~m}, 3 \mathrm{H}), 7.40(\mathrm{dd}, J=8.2,1.6 \mathrm{~Hz}, 1 \mathrm{H}), 7.27-7.24(\mathrm{~m}, 1 \mathrm{H}), 7.12-$ $7.04(\mathrm{~m}, 2 \mathrm{H}), 6.72-6.66(\mathrm{~m}, 1 \mathrm{H}), 1.36$ (d, $J=14.7 \mathrm{~Hz}, 18 \mathrm{H}) .{ }^{13} \mathrm{C}$ NMR (101 MHz, $\left.\mathbf{C D C l}_{3}\right) \delta 162.13(\mathrm{~d}, J=245.4 \mathrm{~Hz}), 142.29,138.04(\mathrm{~d}, J=3.4 \mathrm{~Hz}), 135.59,128.90(\mathrm{~d}$, $J=8.1 \mathrm{~Hz}), 128.45(\mathrm{~d}, J=5.4 \mathrm{~Hz}), 126.99(\mathrm{~d}, J=4.7 \mathrm{~Hz}), 120.63(\mathrm{~d}, J=55.1 \mathrm{~Hz})$, 115.30 (d, $J=21.6 \mathrm{~Hz}), 114.57,106.93$ (d, $J=4.7 \mathrm{~Hz}), 38.62$ (d, $J=69.3 \mathrm{~Hz}), 26.67$. ${ }^{31}$ P NMR (162 MHz, CDCl $) \delta$ 63.33. ${ }^{19}$ F NMR (376 MHz, $\mathbf{C D C l}_{3}$ ) $\delta-117.01$. ATR-FTIR (cm $\left.{ }^{-1}\right): 3131,1511,1470,1400,1218,1179,1135,996,843,815,722$, 662, 631. HRMS m/z (ESI): calcd for $\mathrm{C}_{22} \mathrm{H}_{27} \mathrm{FNOPNa}^{+}$394.1707, found 394.1708.

\section{Di-tert-butyl(6-(4-chlorophenyl)-1H-indol-1-yl)phosphine oxide (3ai)}<smiles></smiles>

3ai
Following the above procedure, the reaction of $\mathbf{1 a}$ $(55.5$

$$
\mathrm{mg}
$$

0.20 mmol), 
(4-chlorophenyl)(mesityl)iodonium triflate (2i) (152.0 mg, $0.30 \mathrm{mmol}), \mathrm{CuO}$ (1.6 mg, $10 \mathrm{~mol} \%)$, in DCE $(6 \mathrm{~mL})$ at $80{ }^{\circ} \mathrm{C}$. After $12 \mathrm{~h}$, purification by column chromatography on silica gel yield 3ai $(64.4 \mathrm{mg}, 83 \%)$ as a white solid. ${ }^{1} \mathbf{H}$ NMR $\left(400 \mathrm{MHz}, \mathbf{C D C l}_{3}\right) \delta 8.83(\mathrm{~s}, 1 \mathrm{H}), 7.66-7.60(\mathrm{~m}, 3 \mathrm{H}), 7.42(\mathrm{dd}, J=8.2,1.6 \mathrm{~Hz}, 1 \mathrm{H})$, $7.38-7.34(\mathrm{~m}, 2 \mathrm{H}), 7.29-7.26(\mathrm{~m}, 1 \mathrm{H}), 6.74-6.66(\mathrm{~m}, 1 \mathrm{H}), 1.36(\mathrm{~d}, J=14.7 \mathrm{~Hz}$, 18H). ${ }^{13}$ C NMR (101 MHz, $\left.\mathbf{C D C l}_{3}\right) \delta 142.31,140.39,135.27,132.61,129.05$ (d, $J=$ $4.5 \mathrm{~Hz}), 128.75(\mathrm{~d}, J=4.8 \mathrm{~Hz}), 128.65,127.16(\mathrm{~d}, J=5.0 \mathrm{~Hz}), 120.83,120.46$, 114.58, 106.97 (d, $J=5.1 \mathrm{~Hz}), 38.64$ (d, $J=69.1 \mathrm{~Hz}), 26.68 .{ }^{31}$ P NMR (162 MHz, $\left.\mathbf{C D C l}_{3}\right) \delta$ 63.42. ATR-FTIR (cm $\left.{ }^{-1}\right): 3125,1683,1466,1400,1174,1139,1091,997$, 814, 664, 615. HRMS m/z (ESI): calcd for $\mathrm{C}_{22} \mathrm{H}_{27} \mathrm{ClNOPNa}^{+}$410.1411, found 410.1412 .

(6-(3-Bromophenyl)-1H-indol-1-yl)di-tert-butylphosphine oxide (3aj)<smiles></smiles>
Following the above procedure, the reaction of 1a $(55.5$ mg, $0.20 \mathrm{mmol}$ ), (3-bromophenyl)(mesityl)iodonium triflate $(2 \mathbf{j})(165.3 \mathrm{mg}, 0.30 \mathrm{mmol}), \mathrm{CuO}(1.6 \mathrm{mg}, 10$ mol\%), in DCE $(6 \mathrm{~mL})$ at $80{ }^{\circ} \mathrm{C}$. After $12 \mathrm{~h}$, purification by column chromatography on silica gel yield 3aj (68.0 mg, 79\%) as a yellow oil. ${ }^{1} \mathbf{H}$ NMR (400 MHz, CDCl $)_{3} \delta 8.81(\mathrm{~s}, 1 \mathrm{H}), 7.82(\mathrm{t}, J=1.8 \mathrm{~Hz}, 1 \mathrm{H}), 7.66-7.61(\mathrm{~m}, 2 \mathrm{H})$, $7.42(\mathrm{dd}, J=8.2,1.5 \mathrm{~Hz}, 2 \mathrm{H}), 7.30-7.26(\mathrm{~m}, 2 \mathrm{H}), 6.75-6.68(\mathrm{~m}, 1 \mathrm{H}), 1.37$ (d, $J=$ $14.7 \mathrm{~Hz}, 18 \mathrm{H}) .{ }^{13} \mathbf{C}$ NMR (101 MHz, $\left.\mathbf{C D C l}_{3}\right) \delta 144.23,142.22,135.15,130.28$, $130.05,129.53,128.96$ (d, $J=5.5 \mathrm{~Hz}), 127.26$ (d, $J=4.7 \mathrm{~Hz}), 126.25,122.70,121.06$, 120.50, 114.78, 106.97 (d, $J=4.7 \mathrm{~Hz}), 38.65$ (d, $J=69.2 \mathrm{~Hz}), 26.69 .{ }^{31}$ P NMR (162 MHz, $\left.\mathbf{C D C l}_{3}\right) \delta$ 63.55. ATR-FTIR $\left(\mathbf{c m}^{-1}\right):$ 3130, 1684, 1514, 1401, 1278, 1237, 1174, 1137, 995, 813， 720，680, 662， 601. HRMS m/z (ESI): calcd for $\mathrm{C}_{22} \mathrm{H}_{27} \mathrm{BrNOPNa}^{+}$454.0906, found 454.0906. 
(6-(4-Bromophenyl)-1H-indol-1-yl)di-tert-butylphosphine oxide (3ak)<smiles></smiles>

Following the above procedure, the reaction of $\mathbf{1 a}$ $(55.5$

$\mathrm{mg}$, 0.20 mmol), (4-bromophenyl)(mesityl)iodonium triflate (2k) (165.3 mg, $0.30 \mathrm{mmol}), \mathrm{CuO}$ (1.6 mg, $10 \mathrm{~mol} \%)$, in

DCE $(6 \mathrm{~mL})$ at $80{ }^{\circ} \mathrm{C}$. After $12 \mathrm{~h}$, purification by column chromatography on silica gel yield 3ak (68.8 mg, 80\%) as a white solid. ${ }^{1} \mathbf{H}$ NMR (400 $\left.\mathbf{M H z}, \mathbf{C D C l}_{3}\right) \delta 8.83(\mathrm{~s}$, $1 \mathrm{H}), 7.63(\mathrm{~d}, J=8.2 \mathrm{~Hz}, 1 \mathrm{H}), 7.59-7.55(\mathrm{~m}, 2 \mathrm{H}), 7.53-7.49(\mathrm{~m}, 2 \mathrm{H}), 7.42(\mathrm{dd}, J=$ 8.2, 1.6 Hz, 1H), 7.27 (dd, $J=3.5,1.4 \mathrm{~Hz}, 1 \mathrm{H}), 6.73-6.65(\mathrm{~m}, 1 \mathrm{H}), 1.36(\mathrm{~d}, J=14.7$ $\mathrm{Hz}, 18 \mathrm{H}) .{ }^{13} \mathbf{C}$ NMR (101 MHz, $\left.\mathbf{C D C l}_{3}\right) \delta$ 142.31, 140.86, 135.26, 131.68, 131.59, 129.02, $128.80(\mathrm{~d}, J=5.3 \mathrm{~Hz}), 127.18(\mathrm{~d}, J=4.7 \mathrm{~Hz}), 120.77,120.49,114.53,106.97$ $(\mathrm{d}, J=4.7 \mathrm{~Hz}), 38.64$ (d, $J=69.2 \mathrm{~Hz}), 26.68 .{ }^{31} \mathbf{P}$ NMR (162 MHz, $\left.\mathbf{C D C l}_{3}\right) \delta 63.43$. ATR-FTIR (cm $\left.{ }^{-1}\right): 3131,1685,1464,1400,1174,1138,1007,812,665,612$. HRMS m/z (ESI): calcd for $\mathrm{C}_{22} \mathrm{H}_{27} \mathrm{BrNOPNa}^{+}$454.0906, found 454.0906.

\section{Di-tert-butyl(6-(4-iodophenyl)-1H-indol-1-yl)phosphine oxide (3al)}<smiles>CC(C)(C)CP(=O)(n1ccc2ccc(-c3ccc(I)cc3)cc21)C(C)(C)C</smiles>

3al

Following the above procedure, the reaction of 1a $(55.5$ $\mathrm{mg}$, 0.20 mmol), (4-iodophenyl)(mesityl)iodonium triflate (2l) (179.5 $\mathrm{mg}, 0.30 \mathrm{mmol}), \mathrm{CuO}(1.6 \mathrm{mg}, 10 \mathrm{~mol} \%)$, in DCE (6 $\mathrm{mL}$ ) at $80{ }^{\circ} \mathrm{C}$. After $12 \mathrm{~h}$, purification by column chromatography on silica gel yield 3al (54.6 mg, 57\%) as a white solid. ${ }^{1} \mathbf{H}$ NMR (400 MHz, $\left.\mathbf{C D C l}_{3}\right) \delta 8.83$ (s, 1H), 7.72 $(\mathrm{d}, J=8.5 \mathrm{~Hz}, 2 \mathrm{H}), 7.63(\mathrm{~d}, J=8.2 \mathrm{~Hz}, 1 \mathrm{H}), 7.49-7.39(\mathrm{~m}, 3 \mathrm{H}), 7.27(\mathrm{dd}, J=3.5$, $1.4 \mathrm{~Hz}, 1 \mathrm{H}), 6.73-6.66(\mathrm{~m}, 1 \mathrm{H}), 1.36(\mathrm{~d}, J=14.7 \mathrm{~Hz}, 18 \mathrm{H}) .{ }^{13} \mathrm{C}$ NMR (101 MHz, $\left.\mathbf{C D C l}_{3}\right) \delta 142.30,141.45,137.58,135.28,129.31,128.85(\mathrm{~d}, J=5.4 \mathrm{~Hz}), 127.20(\mathrm{~d}, J$ $=4.9 \mathrm{~Hz}), 120.70,120.50,114.48,106.97(\mathrm{~d}, J=5.1 \mathrm{~Hz}), 92.21,38.64(\mathrm{~d}, J=69.22$ Hz), 26.68. ${ }^{31}$ P NMR (162 MHz, $\left.\mathbf{C D C l}_{3}\right) \delta$ 63.42. ATR-FTIR (cm $\left.{ }^{-1}\right): 3134,1683$, 1400, 1302, 1173, 1137, 999, 900, 807, 664, 610. HRMS m/z (ESI): calcd for $\mathrm{C}_{22} \mathrm{H}_{27} \mathrm{INOPNa}{ }^{+}$502.0767, found 502.0768. 
Di-tert-butyl(6-(4-(trifluoromethyl)phenyl)-1H-indol-1-yl)phosphine oxide (3am)<smiles></smiles>

Following the above procedure, the reaction of 1a $(55.5$

mg, $\quad 0.20 \quad \mathrm{mmol})$, mesityl(4-(trifluoromethyl)phenyl)iodonium triflate (2m) (162.1 mg, $0.30 \mathrm{mmol}), \mathrm{CuO}(1.6 \mathrm{mg}$, $10 \mathrm{~mol} \%)$, in DCE $(6 \mathrm{~mL})$ at $80{ }^{\circ} \mathrm{C}$. After $12 \mathrm{~h}$, purification by column chromatography on silica gel yield 3am $(64.6 \mathrm{mg}, 77 \%)$ as a white solid. ${ }^{1} \mathbf{H}$ NMR $\left(400 \mathrm{MHz}, \mathbf{C D C l}_{3}\right) \delta 8.89(\mathrm{~s}, 1 \mathrm{H}), 7.81(\mathrm{~d}, J=8.1 \mathrm{~Hz}, 2 \mathrm{H}), 7.65(\mathrm{t}, J=7.7 \mathrm{~Hz}, 3 \mathrm{H})$, $7.47(\mathrm{dd}, J=8.2,1.6 \mathrm{~Hz}, 1 \mathrm{H}), 7.30(\mathrm{dd}, J=3.5,1.5 \mathrm{~Hz}, 1 \mathrm{H}), 6.75-6.69(\mathrm{~m}, 1 \mathrm{H})$, $1.37(\mathrm{~d}, J=14.7 \mathrm{~Hz}, 18 \mathrm{H}) .{ }^{\mathbf{1 3}} \mathbf{C}$ NMR (101 MHz, $\left.\mathbf{C D C l}_{3}\right) \delta 145.47,142.24,134.91$, $129.21(\mathrm{~d}, J=5.5 \mathrm{~Hz}), 128.53(\mathrm{q}, J=32.3 \mathrm{~Hz}), 127.60,127.47(\mathrm{~d}, J=4.9 \mathrm{~Hz}), 125.43$ $(\mathrm{q}, J=3.7 \mathrm{~Hz}), 124.44(\mathrm{q}, J=272.7 \mathrm{~Hz}), 120.96,120.61,115.00,106.98(\mathrm{~d}, J=4.8$ $\mathrm{Hz}), 38.62(\mathrm{~d}, J=69.1 \mathrm{~Hz}), 26.63 .{ }^{31} \mathbf{P}$ NMR (162 $\left.\mathbf{M H z}, \mathbf{C D C l}_{3}\right) \delta 63.61 .{ }^{19} \mathbf{F}$ NMR (376 MHz, $\left.\mathbf{C D C l}_{3}\right) \delta$-62.23. ATR-FTIR (cm $\left.{ }^{-1}\right): 3128,1615,1401,1323,1162,1118$, 1068, 850, 816, 664, 611. HRMS m/z (ESI): calcd for $\mathrm{C}_{23} \mathrm{H}_{27} \mathrm{~F}_{3} \mathrm{NOPNa}^{+}$444.1675, found 444.1676 .

\section{Di-tert-butyl(6-(3-(trifluoromethyl)phenyl)-1H-indol-1-yl)phosphine oxide (3an)}<smiles></smiles>
Following the above procedure, the reaction of 1a $(55.5$ $\mathrm{mg}$, 0.20 mmol), mesityl(3-(trifluoromethyl)phenyl)iodonium triflate (2n) (162.1 mg, $0.30 \mathrm{mmol}), \mathrm{CuO}(1.6 \mathrm{mg}, 10 \mathrm{~mol} \%)$, in DCE $(6 \mathrm{~mL})$ at $80{ }^{\circ} \mathrm{C}$. After $12 \mathrm{~h}$, purification by column chromatography on silica gel yield 3an (65.7 mg, 78\%) as a yellow solid. ${ }^{1} \mathbf{H}$ NMR (400 $\left.\mathbf{M H z}, \mathbf{C D C l}_{3}\right) \delta 8.85$ (s, 1H), $7.91-7.86(\mathrm{~m}, 2 \mathrm{H}), 7.66(\mathrm{~d}, J=8.2 \mathrm{~Hz}, 1 \mathrm{H}), 7.57-7.48(\mathrm{~m}, 2 \mathrm{H}), 7.45(\mathrm{dd}, J=$ 8.2, 1.5 Hz, 1H), 7.29 (dd, $J=3.4,1.3 \mathrm{~Hz}, 1 \mathrm{H}), 6.75-6.69$ (m, 1H), 1.37 (d, $J=14.7$ $\mathrm{Hz}, 18 \mathrm{H}) .{ }^{13} \mathbf{C}$ NMR (101 MHz, $\left.\mathbf{C D C l}_{3}\right) \delta$ 142.87, 142.25, 135.20, 130.98, 130.67, $129.04(\mathrm{~d}, J=5.6 \mathrm{~Hz}), 129.01,127.37(\mathrm{~d}, J=4.6 \mathrm{~Hz}), 124.32(\mathrm{q}, J=275.0 \mathrm{~Hz})$, 124.06 (q, $J=3.5 \mathrm{~Hz}), 123.26(\mathrm{q}, J=3.6 \mathrm{~Hz}), 121.10,120.63,114.90,106.97$ (d, $J=$ $5.0 \mathrm{~Hz}), 38.66(\mathrm{~d}, J=68.9 \mathrm{~Hz}), 26.68 .{ }^{31} \mathbf{P} \mathbf{~ N M R}\left(162 \mathbf{~ M H z}, \mathbf{C D C l}_{3}\right) \delta 63.59 .{ }^{19} \mathbf{F}$ 
NMR (377 MHz, $\left.\mathbf{C D C l}_{3}\right) \delta$-62.39. ATR-FTIR (cm $\left.{ }^{-1}\right): 3124,1684,1400,1336$, 1165, 1124, 804, 663, 602. HRMS m/z (ESI): calcd for $\mathrm{C}_{23} \mathrm{H}_{27} \mathrm{~F}_{3} \mathrm{NOPNa}^{+} 444.1675$, found 444.1676 .

\section{1-(4-(1-(Di-tert-butylphosphoryl)-1H-indol-6-yl)phenyl)ethanone (3ao)}<smiles>CC(=O)c1ccc(-c2ccc3ccn(P(=O)(CC(C)C)OC(C)(C)C)c3c2)cc1</smiles>

Following the above procedure, the reaction of $\mathbf{1 a}$

(55.5 $\quad \mathrm{mg}, \quad 0.20 \quad \mathrm{mmol})$, (4-acetylphenyl)(mesityl)iodonium triflate (2o) (154.3 mg, $0.30 \mathrm{mmol}), \mathrm{CuO}(1.6 \mathrm{mg}, 10 \mathrm{~mol} \%)$,

in DCE $(6 \mathrm{~mL})$ at $80{ }^{\circ} \mathrm{C}$. After $12 \mathrm{~h}$, purification by column chromatography on silica gel yield 3ao (40.3 mg, 51\%) as a yellow solid. ${ }^{1} \mathbf{H}$ NMR (400 $\mathbf{~ M H z , ~} \mathbf{C D C l}_{3}$ ) $\delta 8.92$ (s, 1H), 7.99 (d, $J=8.4 \mathrm{~Hz}, 2 \mathrm{H}), 7.80(\mathrm{~d}, J=8.4 \mathrm{~Hz}, 2 \mathrm{H}), 7.66(\mathrm{~d}, J=8.2 \mathrm{~Hz}, 1 \mathrm{H})$, $7.50(\mathrm{dd}, J=8.2,1.5 \mathrm{~Hz}, 1 \mathrm{H}), 7.30(\mathrm{dd}, J=3.4,1.3 \mathrm{~Hz}, 1 \mathrm{H}), 6.76-6.69$ (m, 1H), $2.63(\mathrm{~s}, 3 \mathrm{H}), 1.37(\mathrm{~d}, J=14.8 \mathrm{~Hz}, 18 \mathrm{H}) .{ }^{13} \mathbf{C}$ NMR (101 MHz, $\left.\mathbf{C D C l}_{3}\right) \delta$ 197.90, 146.64, 142.28, 135.25, 135.08, 129.30 (d, $J=5.3 \mathrm{~Hz}), 128.77,127.52$ (d, $J=4.6 \mathrm{~Hz})$, 127. 42, 121.04, 120.59, 115.04, 107.02 (d, $J=4.9 \mathrm{~Hz}), 38.66$ (d, $J=69.13 \mathrm{~Hz})$, 26.68, 26.64. ${ }^{31}$ P NMR (162 MHz, CDCl $\left.\mathbf{~ M}_{3}\right) \delta$ 63.64. ATR-FTIR (cm $\left.{ }^{-1}\right): 3144,1677$, 1605, 1401, 1270, 1176, 1136, 810, 664, 626. HRMS m/z (ESI): calcd for $\mathrm{C}_{24} \mathrm{H}_{30} \mathrm{NO}_{2} \mathrm{PNa}^{+}$418.1906, found 418.1907.

\section{Ethyl 4-(1-(di-tert-butylphosphoryl)-1H-indol-6-yl)benzoate (3ap)}<smiles>CCOC(=O)c1ccc(-c2ccc3ccn(P(=O)(CC(C)C)CC(C)(C)C)c3c2)cc1</smiles>

Following the above procedure, the reaction of 1a $\quad(55.5 \quad \mathrm{mg}, \quad 0.20 \quad \mathrm{mmol})$, (4-(ethoxycarbonyl)phenyl)(mesityl)iodonium triflate (2p) $(163.3 \mathrm{mg}, 0.30 \mathrm{mmol}), \mathrm{CuO}(1.6$ $\mathrm{mg}, 10 \mathrm{~mol} \%)$, in DCE $(6 \mathrm{~mL})$ at $80{ }^{\circ} \mathrm{C}$. After $12 \mathrm{~h}$, purification by column chromatography on silica gel yield 3ap $(67.5 \mathrm{mg}, 79 \%)$ as a white solid. ${ }^{1} \mathbf{H}$ NMR $\left(400 \mathrm{MHz}, \mathbf{C D C l}_{3}\right) \delta 8.91(\mathrm{~s}, 1 \mathrm{H}), 8.07(\mathrm{~d}, J=8.5 \mathrm{~Hz}, 2 \mathrm{H}), 7.77(\mathrm{~d}, J=8.5 \mathrm{~Hz}, 2 \mathrm{H})$, 7.65 (d, $J=8.2 \mathrm{~Hz}, 1 \mathrm{H}), 7.50$ (dd, $J=8.2,1.6 \mathrm{~Hz}, 1 \mathrm{H}), 7.29$ (dd, $J=3.4,1.4 \mathrm{~Hz}, 1 \mathrm{H})$, $6.74-6.67(\mathrm{~m}, 1 \mathrm{H}), 4.39(\mathrm{q}, J=7.1 \mathrm{~Hz}, 2 \mathrm{H}), 1.41(\mathrm{t}, J=7.1 \mathrm{~Hz}, 3 \mathrm{H}), 1.36(\mathrm{~d}, J=$ 
$14.7 \mathrm{~Hz}, 18 \mathrm{H}) .{ }^{13} \mathbf{C}$ NMR (101 $\left.\mathbf{M H z}, \mathbf{C D C l}_{3}\right) \delta 166.72,146.32,142.24,135.25$, 129.86, 129.18 (d, $J=5.4 \mathrm{~Hz}), 128.47,127.42$ (d, $J=4.6 \mathrm{~Hz}), 127.19,121.07,120.52$, 114.94, $106.98(\mathrm{~d}, J=5.0 \mathrm{~Hz}), 60.79,38.61(\mathrm{~d}, J=69.2 \mathrm{~Hz}), 26.65,14.35 .{ }^{31} \mathbf{P}$ NMR (162 MHz, $\left.\mathbf{C D C l}_{3}\right) \delta$ 63.58. ATR-FTIR (cm $\left.{ }^{-1}\right):$ 3131, 1712, 1606, 1401, 1275, 1171, 1102, 814, 663, 613. HRMS m/z (ESI): calcd for $\mathrm{C}_{25} \mathrm{H}_{32} \mathrm{NO}_{3} \mathrm{PNa}^{+} 448.2012$, found 448.2012 .

\section{Di-tert-butyl(6-(naphthalen-2-yl)-1H-indol-1-yl)phosphine oxide (3aq)}<smiles></smiles>
Following the above procedure, the reaction of 1a $(55.5$ mg, $\quad 0.20 \quad \mathrm{mmol})$, mesityl(naphthalen-2-yl)iodonium triflate (2q) (156.7 $\mathrm{mg}, 0.30 \mathrm{mmol}), \mathrm{CuO}(1.6 \mathrm{mg}, 10 \mathrm{~mol} \%)$, in DCE (6 $\mathrm{mL}$ ) at $80{ }^{\circ} \mathrm{C}$. After $12 \mathrm{~h}$, purification by column chromatography on silica gel yield 3aq (65.4 mg, 81\%) as a white solid. ${ }^{1} \mathbf{H}$ NMR (400 $\left.\mathbf{M H z}, \mathbf{C D C l}_{3}\right) \delta 9.00(\mathrm{~s}, 1 \mathrm{H})$, $8.16(\mathrm{~s}, 1 \mathrm{H}), 7.95-7.81(\mathrm{~m}, 4 \mathrm{H}), 7.71-7.59(\mathrm{~m}, 2 \mathrm{H}), 7.51-7.41(\mathrm{~m}, 2 \mathrm{H}), 7.28(\mathrm{dd}$, $J=3.5,1.4 \mathrm{~Hz}, 1 \mathrm{H}), 6.77-6.66(\mathrm{~m}, 1 \mathrm{H}), 1.38(\mathrm{~d}, J=14.7 \mathrm{~Hz}, 18 \mathrm{H}) .{ }^{13} \mathbf{C}$ NMR (101 MHz, $\left.\mathbf{C D C l}_{3}\right) \delta 142.43,139.30,136.46,133.76,132.39,128.63(\mathrm{~d}, J=5.3 \mathrm{~Hz})$, 128.22, 128.11, 127.53, $127.03(\mathrm{~d}, J=4.7 \mathrm{~Hz}), 126.26,125.96,125.68,125.47$, 121.42, 120.43, 114.98, $106.98(\mathrm{~d}, J=5.1 \mathrm{~Hz}), 38.66(\mathrm{~d}, J=69.3 \mathrm{~Hz}), 26.73 .{ }^{31} \mathbf{P}$ NMR (162 MHz, CDCl $\left.)_{3}\right) \delta$ 63.27. ATR-FTIR (cm $\left.{ }^{-1}\right): ~ 3127,1684,1401,1273,1173$, 1139, 997, 813, 660, 615. HRMS m/z (ESI): calcd for $\mathrm{C}_{26} \mathrm{H}_{30} \mathrm{NOPNa}^{+} 426.1957$, found 426.1956 .

\section{Di-tert-butyl(6-(thiophen-3-yl)-1H-indol-1-yl)phosphine oxide (3ar)}<smiles></smiles>

3ar Following the above procedure, the reaction of $1 \mathbf{a}(55.5$ $\mathrm{mg}, 0.20 \mathrm{mmol}$ ), mesityl(thiophen-3-yl)iodonium triflate (2r) $(143.5 \mathrm{mg}, 0.30 \mathrm{mmol}), \mathrm{CuO}(1.6 \mathrm{mg}, 10 \mathrm{~mol} \%)$, in

DCE $(6 \mathrm{~mL})$ at $80{ }^{\circ} \mathrm{C}$. After $12 \mathrm{~h}$, purification by column chromatography on silica gel yield 3ar (22.0 mg, 31\%) as a yellow oil. ${ }^{1}$ H NMR (400 MHz, $\left.\mathbf{C D C l}_{3}\right) \delta 8.85(\mathrm{~s}, 1 \mathrm{H}), 7.59(\mathrm{~d}, J=8.2 \mathrm{~Hz}, 1 \mathrm{H}), 7.53-7.45(\mathrm{~m}, 3 \mathrm{H}), 7.34(\mathrm{dd}$, 
$J=4.6,3.4 \mathrm{~Hz}, 1 \mathrm{H}), 7.24(\mathrm{dd}, J=3.4,1.2 \mathrm{~Hz}, 1 \mathrm{H}), 6.70-6.65(\mathrm{~m}, 1 \mathrm{H}), 1.36(\mathrm{~d}, J=$ $14.7 \mathrm{~Hz}, 18 \mathrm{H}) .{ }^{13} \mathbf{C}$ NMR (101 MHz, $\left.\mathbf{C D C l}_{3}\right) \delta 143.20,142.25,131.36,128.37$ (d, $J$ $=5.4 \mathrm{~Hz}), 126.89,126.82,125.59,120.58,120.31,119.60,113.99,107.03$ (d, $J=5.1$ $\mathrm{Hz}), 38.61(\mathrm{~d}, J=69.3 \mathrm{~Hz}), 26.68 .{ }^{31} \mathbf{P}$ NMR (162 MHz, $\left.\mathbf{C D C l}_{3}\right) \delta 63.28$. ATR-FTIR (cm $\left.{ }^{-1}\right):$ 3127, 1685, 1401, 1279, 1135, 996, 858, 813, 781, 725, 661, 600. HRMS m/z (ESI): calcd for $\mathrm{C}_{20} \mathrm{H}_{26} \mathrm{NOPSNa}^{+}$382.1365, found 382.1367.

\section{(E)-Di-tert-butyl(6-styryl-1H-indol-1-yl)phosphine oxide (10)}<smiles>CCCP(=O)(n1ccc2ccc(/C=C/c3ccccc3)cc21)C(C)(C)C</smiles>

10

Following the above procedure, the reaction of $1 \mathrm{a}(55.5$ $\mathrm{mg}, 0.20 \mathrm{mmol}),(E)-3$-phenyl-1-(o-tolyl)allyl triflate (9) (141.1 mg, $0.30 \mathrm{mmol}), \mathrm{CuO}(1.6 \mathrm{mg}, 10 \mathrm{~mol} \%)$, in DCE $(6 \mathrm{~mL})$ at $80{ }^{\circ} \mathrm{C}$. After $12 \mathrm{~h}$, purification by column chromatography on silica gel yield $10(19.0 \mathrm{mg}, 25 \%)$ as a white solid. ${ }^{1} \mathbf{H}$ NMR (400 MHz, CDCl $) \delta 8.71(\mathrm{~s}, 1 \mathrm{H}), 7.57-7.49(\mathrm{~m}, 3 \mathrm{H}), 7.43(\mathrm{~d}, J=8.2 \mathrm{~Hz}$, 1H), 7.34 (t, $J=7.6 \mathrm{~Hz}, 2 \mathrm{H}), 7.29-7.14(\mathrm{~m}, 4 \mathrm{H}), 6.67$ (s, 1H), 1.37 (d, J=14.7 Hz, 18H). ${ }^{13}$ C NMR (101 MHz, $\left.\mathbf{C D C l}_{3}\right) \delta$ 142.20, 137.86, 132.99, 132.97, 130.03, $129.02(\mathrm{~d}, J=5.2 \mathrm{~Hz}), 128.58,127.08,127.05,127.00$ (d, $J=4.9 \mathrm{~Hz}), 126.31,120.25$, 114.75, $107.24(\mathrm{~d}, J=5.1 \mathrm{~Hz}), 38.63$ (d, $J=69.3 \mathrm{~Hz}), 26.70 .{ }^{31}$ P NMR (162 MHz, $\left.\mathbf{C D C l}_{3}\right) \delta$ 63.29. ATR-FTIR (cm $\left.{ }^{-1}\right): 3127,1686,1655,1618,1560,1400,1128,812$, 746, 690, 662, 618. HRMS m/z (ESI): calcd for $\mathrm{C}_{24} \mathrm{H}_{30} \mathrm{NOPNa}^{+} 402.1957$, found 402.1958 .

\section{Further Applications}

\subsection{Gram scale reaction}

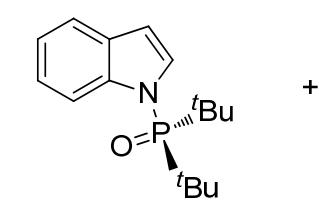

1a $(4.5 \mathrm{mmol}, 1.248 \mathrm{~g})$
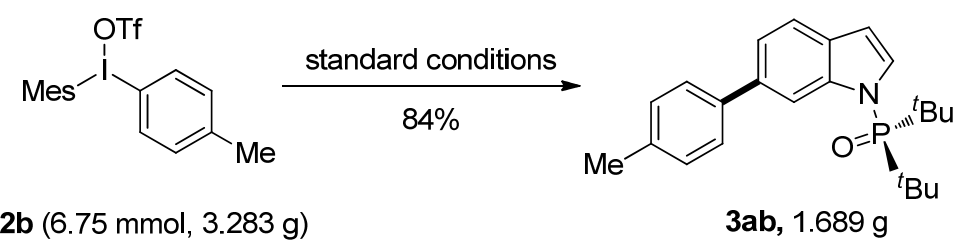

$3 \mathrm{ab}, 1.689 \mathrm{~g}$

To an oven dried $250 \mathrm{~mL}$ Schlenk tube was charged with a stirred bar, indole substrates 1a (1.248 g, $4.5 \mathrm{mmol})$, DCE (135 mL) and keep stirred for $5 \mathrm{~min}$. The 
tube was purged with Ar for three times, then mesityl( $p$-tolyl)iodonium triflate (2b) (3.283 g, $6.75 \mathrm{mmol}), \mathrm{CuO}(35.8 \mathrm{mg}, 10 \mathrm{~mol} \%)$ were added into the solution of indoles in DCE. The formed mixture was stirred at $80{ }^{\circ} \mathrm{C}$ under $\mathrm{Ar}$ for $12 \mathrm{~h}$ as monitored by TLC. The solution was then cooled to rt, and the solvent was removed under vaccum directly. The crude product was purified by column chromatography on silica gel (Petroleum ether : Ethyl acetate $=3: 1$ ) to afford the pure products 3ab $(1.689 \mathrm{~g}, 84 \%)$.

\subsection{Removal of the direction group}

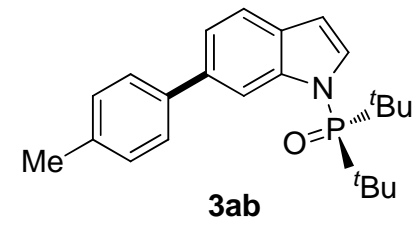

To a solution of di-tert-butyl(6-(p-tolyl)-1H-indol-1-yl)phosphine oxide (3ab, $73.5 \mathrm{mg}, 0.20 \mathrm{mmol})$ in $4 \mathrm{ml}$ anhydrous THF was added an excess of $\mathrm{LiAlH}_{4}(27 \mathrm{mg}$, $0.7 \mathrm{mmol})$. The mixture was heated to $100{ }^{\circ} \mathrm{C}$ and reaction progress was monitored by GC over several hours. After $3 \mathrm{~h}$ a second portion of $\mathrm{LiAlH}_{4}(38 \mathrm{mg}, 1.0 \mathrm{mmol})$ was added. After another $29 \mathrm{~h}$ the reaction mixture was cooled to room temperature and then to $0{ }^{\circ} \mathrm{C}$ in an ice bath. Excess $\mathrm{LiAlH}_{4}$ was quenched by the careful addition of $\mathrm{H}_{2} \mathrm{O}$ (4 ml) over several minutes. When all effervescence had ceased, the mixture was transferred to a separatory funnel containing $\mathrm{H}_{2} \mathrm{O}(20 \mathrm{ml})$ and extracted with EtOAc ( 3 x $20 \mathrm{ml})$. The combined organics were dried $\left(\mathrm{Na}_{2} \mathrm{SO}_{4}\right)$ and evaporated to provide a white solid. Purification by flash column (EtOAc : hexane=1:50) provided 6 (37 mg, 89\%) as a white solid. ${ }^{\mathbf{1}} \mathbf{H}$ NMR (400 $\left.\mathbf{~ M H z , ~} \mathbf{C D C l}_{3}\right) \delta 8.12(\mathrm{~s}, 1 \mathrm{H}), 7.68(\mathrm{~d}, J=8.2$ $\mathrm{Hz}, 1 \mathrm{H}), 7.55(\mathrm{~d}, J=8.3 \mathrm{~Hz}, 3 \mathrm{H}), 7.38(\mathrm{dd}, J=8.2,1.5 \mathrm{~Hz}, 1 \mathrm{H}), 7.28-7.23(\mathrm{~m}, 2 \mathrm{H})$, $7.22-7.17(\mathrm{~m}, 1 \mathrm{H}), 6.60-6.54(\mathrm{~m}, 1 \mathrm{H}), 2.40$ (s, 3H). ${ }^{13} \mathbf{C}$ NMR (101 MHz, $\left.\mathbf{C D C l}_{3}\right)$ $\delta 139.39,136.35,136.27,135.50,129.40,127.20,126.98,124.64,120.82,119.68$, 109.27, 102.49, 21.06. ATR-FTIR (cm $\left.{ }^{-1}\right):$ 3130, 1684, 1619, 1400, 1346, 1095, 810, 722, 611. EI-MS (m/z, relative intensity): 207 ( $\left.\mathrm{M}^{+}, 100\right), 191$ (12), 178 (14), 164 (6), 103 (11), 89 (13), $76(5)$. 


\subsection{Generation of 2,6-diarylated indole}<smiles>Cc1ccc(-c2ccc3cc[nH]c3c2)cc1</smiles>

6<smiles>Cc1ccc(-c2ccc3cc(-c4ccccc4)[nH]c3c2)cc1</smiles>

7

Modified by Sanford's conditions, ${ }^{3}$ 6-(p-tolyl)-1H-indole $(20.7 \mathrm{mg}, 0.1 \mathrm{mmol}$, 1.0 equiv) and $\mathrm{IMesPd}(\mathrm{OAc})_{2}(2.5 \mathrm{mg}, 0.005 \mathrm{mmol}, 0.05$ equiv) were dissolved in $\mathrm{AcOH}(1.0 \mathrm{~mL})$, and the resulting solution was stirred at $25^{\circ} \mathrm{C}$ for $\sim 5 \mathrm{~min} .\left[\mathrm{Ph}_{2} \mathrm{I}\right] \mathrm{BF}_{4}$ (110.4 mg, $0.3 \mathrm{mmol}, 3.0$ equiv) was added, and the reaction was stirred for $12 \mathrm{~h}$ at 25 ${ }^{\circ} \mathrm{C}$, then rise the temperature to $60{ }^{\circ} \mathrm{C}$ and keep stirred for another $5.5 \mathrm{~h}$. The reaction mixture was filtered through a plug of Celite and then evaporated to dryness. The resulting oil was dissolved in $\mathrm{CH}_{2} \mathrm{Cl}_{2}(20 \mathrm{~mL})$ and extracted with aqueous $\mathrm{NaHCO}_{3}(2$ x $20 \mathrm{~mL}$ ). The organic layer was dried with $\mathrm{MgSO}_{4}$, concentrated, and the product was purified by chromatography on silica gel ((EtOAc : hexane $=1: 50)$ provided 7 (16.7 mg, 59\%) as a white solid. ${ }^{1} \mathbf{H}$ NMR (400 MHz, $\left.\mathbf{C D C l}_{3}\right) \delta 8.35$ (s, 1H), 7.66 (dd, $J=7.9,2.6 \mathrm{~Hz}, 3 \mathrm{H}), 7.59-7.52$ (m, 3H), 7.44 (t, $J=7.7 \mathrm{~Hz}, 2 \mathrm{H}), 7.37$ (dd, $J=$ 8.2, 1.3 Hz, 1H), 7.32 (t, $J=7.4 \mathrm{~Hz}, 1 \mathrm{H}), 7.25(\mathrm{~d}, J=9.0 \mathrm{~Hz}, 2 \mathrm{H}), 6.83(\mathrm{~d}, J=1.2 \mathrm{~Hz}$, 1H), 2.40 (s, 3H). ${ }^{13}$ C NMR (101 MHz, $\left.\mathbf{C D C l}_{3}\right) \delta 139.33,138.38,137.42,136.34$, $135.82,132.30,129.44,129.03,128.44,127.71,127.12,125.09,120.76,120.13$, 109.09, 99.88, 21.07. ATR-FTIR (cm $\left.{ }^{-1}\right): 3131,1683,1401,1317,863,810,759,740$, 686. HRMS m/z (ESI): calcd for $\mathrm{C}_{21} \mathrm{H}_{18} \mathrm{~N}(\mathrm{M}+\mathrm{H})^{+}$284.1434, found 284.1434.

\subsection{Generation of 3,6-diarylated indole}<smiles>Cc1ccc(-c2ccc3cc[nH]c3c2)cc1</smiles>

6

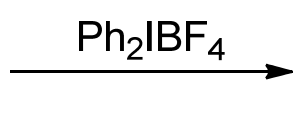<smiles>Cc1ccc(-c2ccc3c(-c4ccccc4)c[nH]c3c2)cc1</smiles>

8 
Modified by Gaunt's conditions, ${ }^{4}$ 2,6-Di-tert-butylpyridine (34 $\left.\mu \mathrm{L}, 0.15 \mathrm{mmol}\right)$, $\mathrm{Cu}(\mathrm{OTf})_{2}(3.6 \mathrm{mg}, 0.01 \mathrm{mmol})$ and $\left[\mathrm{Ph}_{2} \mathrm{I}\right] \mathrm{BF}_{4}(55.2 \mathrm{mg}, 0.15 \mathrm{mmol})$ were added to a solution of 6-(p-tolyl)-1H-indole $(20.7 \mathrm{mg}, 0.1 \mathrm{mmol})$ in DCE $(2 \mathrm{ml})$. The reaction was stirred at $60{ }^{\circ} \mathrm{C}$ for $70 \mathrm{~h}$ then diluted with $\mathrm{CH}_{2} \mathrm{Cl}_{2}(10 \mathrm{ml})$ and washed with satd. $\mathrm{NaHCO}_{3}$. The aqueous layer was further extracted with $\mathrm{CH}_{2} \mathrm{Cl}_{2}(10 \mathrm{ml})$ and the combined organics dried $\left(\mathrm{MgSO}_{4}\right)$, and evaporated in vacuo. Purification by flash column chromatography on silica gel (EtOAc : hexane=1:20) provided 8 (20 mg, 71\%) as a yellow solid. ${ }^{\mathbf{1}} \mathbf{H}$ NMR (400 $\left.\mathbf{~ M H z , ~} \mathbf{C D C l}_{3}\right) \delta 8.28(\mathrm{~s}, 1 \mathrm{H}), 7.99$ (d, $J=8.4$ Hz, 1H), $7.75-7.67$ (m, 2H), 7.62 (d, J=0.9 Hz, 1H), 7.58 (d, J=8.1 Hz, 2H), 7.49 $-7.43(\mathrm{~m}, 3 \mathrm{H}), 7.40(\mathrm{~d}, J=2.5 \mathrm{~Hz}, 1 \mathrm{H}), 7.34-7.27(\mathrm{~m}, 3 \mathrm{H}), 2.42(\mathrm{~s}, 3 \mathrm{H}) .{ }^{13} \mathbf{C} \mathbf{N M R}$ (101 MHz, $\mathbf{C D C l}$ ) $\delta$ 139.13, 137.25, 136.45, 135.91, 135.46, 129.45, 128.77, 127.41, 127.19, 126.01, 124.86, 122.20, 120.12, 119.98, 118.29, 109.60, 21.08. ATR-FTIR $\left(\mathbf{c m}^{-1}\right):$ 3130, 1684, 1400, 1116, 958, 805, 765, 751, 698. HRMS m/z (ESI): calcd for $\mathrm{C}_{21} \mathrm{H}_{18} \mathrm{~N}(\mathrm{M}+\mathrm{H})^{+} 284.1434$, found 284.1429.

\section{Crystallographic Data}<smiles>CCCCC(C)(C)P(=O)(CC(C)(C)C)n1ccc2ccc(-c3ccccc3)cc21</smiles>
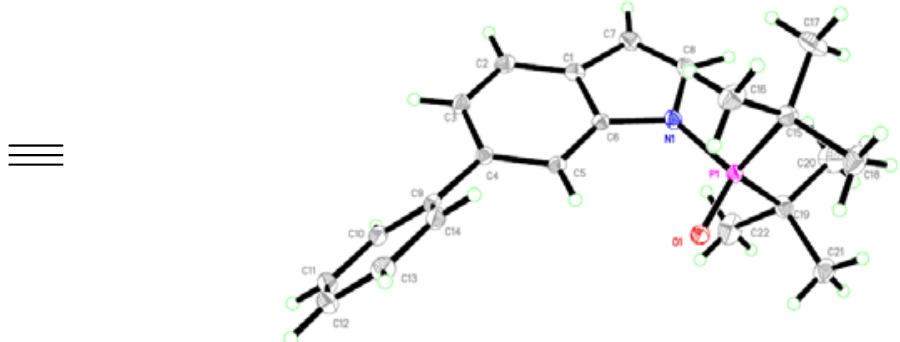

$\mathrm{C}(1)-\mathrm{C}(2)$

$1.405(2)$

C(4)-C(9)

$1.489(2)$

$\mathrm{C}(1)-\mathrm{C}(6)$

$1.410(2)$

$\mathrm{C}(5)-\mathrm{C}(6)$

1.393(2)

$\mathrm{C}(1)-\mathrm{C}(7)$

$1.429(2)$

$\mathrm{C}(5)-\mathrm{H}(5)$

0.9300

$\mathrm{C}(2)-\mathrm{C}(3)$

$1.375(2)$

$\mathrm{C}(6)-\mathrm{N}(1)$

1.410(2)

$\mathrm{C}(2)-\mathrm{H}(2)$

0.9300

$\mathrm{C}(7)-\mathrm{C}(8)$

1.343(2)

$\mathrm{C}(3)-\mathrm{C}(4)$

1.411(2)

$\mathrm{C}(7)-\mathrm{H}(7)$

0.9300

$\mathrm{C}(3)-\mathrm{H}(3)$

0.9300

$\mathrm{C}(8)-\mathrm{N}(1)$

1.405(2)

C(4)-C(5)

1.391(2)

$\mathrm{C}(8)-\mathrm{H}(8)$

0.9300 


\begin{tabular}{|c|c|c|c|}
\hline$C(9)-C(10)$ & $1.395(2)$ & $\mathrm{O}(1)-\mathrm{P}(1)$ & $1.4797(12)$ \\
\hline$C(9)-C(14)$ & $1.396(2)$ & & \\
\hline$C(10)-C(11)$ & $1.389(2)$ & $C(2)-C(1)-C(6)$ & $119.31(15)$ \\
\hline $\mathrm{C}(10)-\mathrm{H}(10)$ & 0.9300 & $C(2)-C(1)-C(7)$ & $133.34(15)$ \\
\hline$C(11)-C(12)$ & $1.386(3)$ & $C(6)-C(1)-C(7)$ & $107.35(14)$ \\
\hline $\mathrm{C}(11)-\mathrm{H}(11)$ & 0.9300 & $\mathrm{C}(3)-\mathrm{C}(2)-\mathrm{C}(1)$ & $118.77(15)$ \\
\hline$C(12)-C(13)$ & $1.387(3)$ & $\mathrm{C}(3)-\mathrm{C}(2)-\mathrm{H}(2)$ & 120.6 \\
\hline $\mathrm{C}(12)-\mathrm{H}(12)$ & 0.9300 & $\mathrm{C}(1)-\mathrm{C}(2)-\mathrm{H}(2)$ & 120.6 \\
\hline$C(13)-C(14)$ & $1.386(2)$ & $C(2)-C(3)-C(4)$ & $121.68(15)$ \\
\hline $\mathrm{C}(13)-\mathrm{H}(13)$ & 0.9300 & $\mathrm{C}(2)-\mathrm{C}(3)-\mathrm{H}(3)$ & 119.2 \\
\hline $\mathrm{C}(14)-\mathrm{H}(14)$ & 0.9300 & $\mathrm{C}(4)-\mathrm{C}(3)-\mathrm{H}(3)$ & 119.2 \\
\hline$C(15)-C(17)$ & $1.530(2)$ & $C(5)-C(4)-C(3)$ & $120.23(14)$ \\
\hline $\mathrm{C}(15)-\mathrm{C}(18)$ & $1.534(2)$ & $\mathrm{C}(5)-\mathrm{C}(4)-\mathrm{C}(9)$ & $119.56(14)$ \\
\hline$C(15)-C(16)$ & $1.539(2)$ & $\mathrm{C}(3)-\mathrm{C}(4)-\mathrm{C}(9)$ & $120.21(14)$ \\
\hline $\mathrm{C}(15)-\mathrm{P}(1)$ & $1.8446(17)$ & $C(4)-C(5)-C(6)$ & $118.07(14)$ \\
\hline $\mathrm{C}(16)-\mathrm{H}(16 \mathrm{~A})$ & 0.9600 & $\mathrm{C}(4)-\mathrm{C}(5)-\mathrm{H}(5)$ & 121.0 \\
\hline $\mathrm{C}(16)-\mathrm{H}(16 \mathrm{~B})$ & 0.9600 & $\mathrm{C}(6)-\mathrm{C}(5)-\mathrm{H}(5)$ & 121.0 \\
\hline $\mathrm{C}(16)-\mathrm{H}(16 \mathrm{C})$ & 0.9600 & $C(5)-C(6)-C(1)$ & $121.91(14)$ \\
\hline $\mathrm{C}(17)-\mathrm{H}(17 \mathrm{~A})$ & 0.9600 & $C(5)-C(6)-N(1)$ & $130.33(14)$ \\
\hline $\mathrm{C}(17)-\mathrm{H}(17 \mathrm{~B})$ & 0.9600 & $\mathrm{C}(1)-\mathrm{C}(6)-\mathrm{N}(1)$ & $107.75(14)$ \\
\hline $\mathrm{C}(17)-\mathrm{H}(17 \mathrm{C})$ & 0.9600 & $\mathrm{C}(8)-\mathrm{C}(7)-\mathrm{C}(1)$ & $107.45(14)$ \\
\hline $\mathrm{C}(18)-\mathrm{H}(18 \mathrm{~A})$ & 0.9600 & $\mathrm{C}(8)-\mathrm{C}(7)-\mathrm{H}(7)$ & 126.3 \\
\hline $\mathrm{C}(18)-\mathrm{H}(18 \mathrm{~B})$ & 0.9600 & $\mathrm{C}(1)-\mathrm{C}(7)-\mathrm{H}(7)$ & 126.3 \\
\hline $\mathrm{C}(18)-\mathrm{H}(18 \mathrm{C})$ & 0.9600 & $\mathrm{C}(7)-\mathrm{C}(8)-\mathrm{N}(1)$ & $111.10(15)$ \\
\hline $\mathrm{C}(19)-\mathrm{C}(20)$ & $1.531(3)$ & $\mathrm{C}(7)-\mathrm{C}(8)-\mathrm{H}(8)$ & 124.5 \\
\hline$C(19)-C(22)$ & $1.532(2)$ & $\mathrm{N}(1)-\mathrm{C}(8)-\mathrm{H}(8)$ & 124.5 \\
\hline $\mathrm{C}(19)-\mathrm{C}(21)$ & $1.541(3)$ & $\mathrm{C}(10)-\mathrm{C}(9)-\mathrm{C}(14)$ & $118.50(15)$ \\
\hline $\mathrm{C}(19)-\mathrm{P}(1)$ & $1.8518(17)$ & $C(10)-C(9)-C(4)$ & $121.04(14)$ \\
\hline $\mathrm{C}(20)-\mathrm{H}(20 \mathrm{~A})$ & 0.9600 & $\mathrm{C}(14)-\mathrm{C}(9)-\mathrm{C}(4)$ & $120.46(14)$ \\
\hline $\mathrm{C}(20)-\mathrm{H}(20 \mathrm{~B})$ & 0.9600 & $C(11)-C(10)-C(9)$ & $120.64(16)$ \\
\hline $\mathrm{C}(20)-\mathrm{H}(20 \mathrm{C})$ & 0.9600 & $\mathrm{C}(11)-\mathrm{C}(10)-\mathrm{H}(10)$ & 119.7 \\
\hline $\mathrm{C}(21)-\mathrm{H}(21 \mathrm{~A})$ & 0.9600 & $\mathrm{C}(9)-\mathrm{C}(10)-\mathrm{H}(10)$ & 119.7 \\
\hline $\mathrm{C}(21)-\mathrm{H}(21 \mathrm{~B})$ & 0.9600 & $\mathrm{C}(12)-\mathrm{C}(11)-\mathrm{C}(10)$ & $120.25(16)$ \\
\hline $\mathrm{C}(21)-\mathrm{H}(21 \mathrm{C})$ & 0.9600 & $\mathrm{C}(12)-\mathrm{C}(11)-\mathrm{H}(11)$ & 119.9 \\
\hline $\mathrm{C}(22)-\mathrm{H}(22 \mathrm{~A})$ & 0.9600 & $\mathrm{C}(10)-\mathrm{C}(11)-\mathrm{H}(11)$ & 119.9 \\
\hline $\mathrm{C}(22)-\mathrm{H}(22 \mathrm{~B})$ & 0.9600 & $\mathrm{C}(11)-\mathrm{C}(12)-\mathrm{C}(13)$ & $119.65(16)$ \\
\hline $\mathrm{C}(22)-\mathrm{H}(22 \mathrm{C})$ & 0.9600 & $\mathrm{C}(11)-\mathrm{C}(12)-\mathrm{H}(12)$ & 120.2 \\
\hline $\mathrm{N}(1)-\mathrm{P}(1)$ & $1.7096(14)$ & $\mathrm{C}(13)-\mathrm{C}(12)-\mathrm{H}(12)$ & 120.2 \\
\hline
\end{tabular}




\begin{tabular}{|c|c|c|c|}
\hline$C(14)-C(13)-C(12)$ & $120.16(16)$ & $C(22)-C(19)-C(21)$ & $107.87(16)$ \\
\hline $\mathrm{C}(14)-\mathrm{C}(13)-\mathrm{H}(13)$ & 119.9 & $\mathrm{C}(20)-\mathrm{C}(19)-\mathrm{P}(1)$ & $117.47(13)$ \\
\hline $\mathrm{C}(12)-\mathrm{C}(13)-\mathrm{H}(13)$ & 119.9 & $\mathrm{C}(22)-\mathrm{C}(19)-\mathrm{P}(1)$ & $105.51(12)$ \\
\hline$C(13)-C(14)-C(9)$ & $120.79(16)$ & $\mathrm{C}(21)-\mathrm{C}(19)-\mathrm{P}(1)$ & $106.64(12)$ \\
\hline $\mathrm{C}(13)-\mathrm{C}(14)-\mathrm{H}(14)$ & 119.6 & $\mathrm{C}(19)-\mathrm{C}(20)-\mathrm{H}(20 \mathrm{~A})$ & 109.5 \\
\hline $\mathrm{C}(9)-\mathrm{C}(14)-\mathrm{H}(14)$ & 119.6 & $\mathrm{C}(19)-\mathrm{C}(20)-\mathrm{H}(20 \mathrm{~B})$ & 109.5 \\
\hline $\mathrm{C}(17)-\mathrm{C}(15)-\mathrm{C}(18)$ & $110.14(16)$ & $\mathrm{H}(20 \mathrm{~A})-\mathrm{C}(20)-\mathrm{H}(20 \mathrm{~B})$ & 109.5 \\
\hline$C(17)-C(15)-C(16)$ & $108.10(15)$ & $\mathrm{C}(19)-\mathrm{C}(20)-\mathrm{H}(20 \mathrm{C})$ & 109.5 \\
\hline$C(18)-C(15)-C(16)$ & $109.10(14)$ & $\mathrm{H}(20 \mathrm{~A})-\mathrm{C}(20)-\mathrm{H}(20 \mathrm{C})$ & 109.5 \\
\hline $\mathrm{C}(17)-\mathrm{C}(15)-\mathrm{P}(1)$ & $116.09(12)$ & $\mathrm{H}(20 \mathrm{~B})-\mathrm{C}(20)-\mathrm{H}(20 \mathrm{C})$ & 109.5 \\
\hline $\mathrm{C}(18)-\mathrm{C}(15)-\mathrm{P}(1)$ & $109.16(12)$ & $\mathrm{C}(19)-\mathrm{C}(21)-\mathrm{H}(21 \mathrm{~A})$ & 109.5 \\
\hline $\mathrm{C}(16)-\mathrm{C}(15)-\mathrm{P}(1)$ & $103.92(12)$ & $\mathrm{C}(19)-\mathrm{C}(21)-\mathrm{H}(21 \mathrm{~B})$ & 109.5 \\
\hline $\mathrm{C}(15)-\mathrm{C}(16)-\mathrm{H}(16 \mathrm{~A})$ & 109.5 & $\mathrm{H}(21 \mathrm{~A})-\mathrm{C}(21)-\mathrm{H}(21 \mathrm{~B})$ & 109.5 \\
\hline $\mathrm{C}(15)-\mathrm{C}(16)-\mathrm{H}(16 \mathrm{~B})$ & 109.5 & $\mathrm{C}(19)-\mathrm{C}(21)-\mathrm{H}(21 \mathrm{C})$ & 109.5 \\
\hline $\mathrm{H}(16 \mathrm{~A})-\mathrm{C}(16)-\mathrm{H}(16 \mathrm{~B})$ & 109.5 & $\mathrm{H}(21 \mathrm{~A})-\mathrm{C}(21)-\mathrm{H}(21 \mathrm{C})$ & 109.5 \\
\hline $\mathrm{C}(15)-\mathrm{C}(16)-\mathrm{H}(16 \mathrm{C})$ & 109.5 & $\mathrm{H}(21 \mathrm{~B})-\mathrm{C}(21)-\mathrm{H}(21 \mathrm{C})$ & 109.5 \\
\hline $\mathrm{H}(16 \mathrm{~A})-\mathrm{C}(16)-\mathrm{H}(16 \mathrm{C})$ & 109.5 & $\mathrm{C}(19)-\mathrm{C}(22)-\mathrm{H}(22 \mathrm{~A})$ & 109.5 \\
\hline $\mathrm{H}(16 \mathrm{~B})-\mathrm{C}(16)-\mathrm{H}(16 \mathrm{C})$ & 109.5 & $\mathrm{C}(19)-\mathrm{C}(22)-\mathrm{H}(22 \mathrm{~B})$ & 109.5 \\
\hline $\mathrm{C}(15)-\mathrm{C}(17)-\mathrm{H}(17 \mathrm{~A})$ & 109.5 & $\mathrm{H}(22 \mathrm{~A})-\mathrm{C}(22)-\mathrm{H}(22 \mathrm{~B})$ & 109.5 \\
\hline $\mathrm{C}(15)-\mathrm{C}(17)-\mathrm{H}(17 \mathrm{~B})$ & 109.5 & $\mathrm{C}(19)-\mathrm{C}(22)-\mathrm{H}(22 \mathrm{C})$ & 109.5 \\
\hline $\mathrm{H}(17 \mathrm{~A})-\mathrm{C}(17)-\mathrm{H}(17 \mathrm{~B})$ & 109.5 & $\mathrm{H}(22 \mathrm{~A})-\mathrm{C}(22)-\mathrm{H}(22 \mathrm{C})$ & 109.5 \\
\hline $\mathrm{C}(15)-\mathrm{C}(17)-\mathrm{H}(17 \mathrm{C})$ & 109.5 & $\mathrm{H}(22 \mathrm{~B})-\mathrm{C}(22)-\mathrm{H}(22 \mathrm{C})$ & 109.5 \\
\hline $\mathrm{H}(17 \mathrm{~A})-\mathrm{C}(17)-\mathrm{H}(17 \mathrm{C})$ & 109.5 & $\mathrm{C}(8)-\mathrm{N}(1)-\mathrm{C}(6)$ & $106.36(13)$ \\
\hline $\mathrm{H}(17 \mathrm{~B})-\mathrm{C}(17)-\mathrm{H}(17 \mathrm{C})$ & 109.5 & $\mathrm{C}(8)-\mathrm{N}(1)-\mathrm{P}(1)$ & $126.00(11)$ \\
\hline $\mathrm{C}(15)-\mathrm{C}(18)-\mathrm{H}(18 \mathrm{~A})$ & 109.5 & $\mathrm{C}(6)-\mathrm{N}(1)-\mathrm{P}(1)$ & $127.58(11)$ \\
\hline $\mathrm{C}(15)-\mathrm{C}(18)-\mathrm{H}(18 \mathrm{~B})$ & 109.5 & $\mathrm{O}(1)-\mathrm{P}(1)-\mathrm{N}(1)$ & $110.45(6)$ \\
\hline $\mathrm{H}(18 \mathrm{~A})-\mathrm{C}(18)-\mathrm{H}(18 \mathrm{~B})$ & 109.5 & $\mathrm{O}(1)-\mathrm{P}(1)-\mathrm{C}(15)$ & $110.23(7)$ \\
\hline $\mathrm{C}(15)-\mathrm{C}(18)-\mathrm{H}(18 \mathrm{C})$ & 109.5 & $\mathrm{~N}(1)-\mathrm{P}(1)-\mathrm{C}(15)$ & $104.60(7)$ \\
\hline $\mathrm{H}(18 \mathrm{~A})-\mathrm{C}(18)-\mathrm{H}(18 \mathrm{C})$ & 109.5 & $\mathrm{O}(1)-\mathrm{P}(1)-\mathrm{C}(19)$ & $109.59(7)$ \\
\hline $\mathrm{H}(18 \mathrm{~B})-\mathrm{C}(18)-\mathrm{H}(18 \mathrm{C})$ & 109.5 & N(1)-P(1)-C(19) & $106.21(7)$ \\
\hline$C(20)-C(19)-C(22)$ & $110.91(17)$ & $C(15)-P(1)-C(19)$ & $115.53(8)$ \\
\hline$C(20)-C(19)-C(21)$ & $108.02(16)$ & & \\
\hline
\end{tabular}


<smiles>CC(C)(C)CP(=O)(n1ccc2c(Br)cc(-c3ccccc3)cc21)C(C)(C)C</smiles>

\begin{tabular}{|c|c|c|c|}
\hline $\operatorname{Br}(1)-C(9)$ & $1.904(2)$ & $C(15)-C(16)$ & $1.543(3)$ \\
\hline $\mathrm{Br}(2)-\mathrm{C}(33)$ & $1.902(2)$ & $\mathrm{C}(15)-\mathrm{P}(1)$ & $1.849(2)$ \\
\hline $\mathrm{C}(1)-\mathrm{C}(2)$ & $1.382(3)$ & $\mathrm{C}(16)-\mathrm{H}(16 \mathrm{~A})$ & 0.9600 \\
\hline$C(1)-C(6)$ & $1.391(3)$ & $\mathrm{C}(16)-\mathrm{H}(16 \mathrm{~B})$ & 0.9600 \\
\hline $\mathrm{C}(1)-\mathrm{H}(1)$ & 0.9300 & $\mathrm{C}(16)-\mathrm{H}(16 \mathrm{C})$ & 0.9600 \\
\hline $\mathrm{C}(2)-\mathrm{C}(3)$ & $1.386(3)$ & $\mathrm{C}(17)-\mathrm{H}(17 \mathrm{~A})$ & 0.9600 \\
\hline $\mathrm{C}(2)-\mathrm{H}(2)$ & 0.9300 & $\mathrm{C}(17)-\mathrm{H}(17 \mathrm{~B})$ & 0.9600 \\
\hline$C(3)-C(4)$ & $1.377(3)$ & $\mathrm{C}(17)-\mathrm{H}(17 \mathrm{C})$ & 0.9600 \\
\hline $\mathrm{C}(3)-\mathrm{H}(3)$ & 0.9300 & $\mathrm{C}(18)-\mathrm{H}(18 \mathrm{~A})$ & 0.9600 \\
\hline$C(4)-C(5)$ & $1.376(3)$ & $\mathrm{C}(18)-\mathrm{H}(18 \mathrm{~B})$ & 0.9600 \\
\hline $\mathrm{C}(4)-\mathrm{H}(4)$ & 0.9300 & $\mathrm{C}(18)-\mathrm{H}(18 \mathrm{C})$ & 0.9600 \\
\hline$C(5)-C(6)$ & $1.407(3)$ & $\mathrm{C}(19)-\mathrm{C}(22)$ & $1.534(3)$ \\
\hline $\mathrm{C}(5)-\mathrm{H}(5)$ & 0.9300 & $C(19)-C(20)$ & $1.537(3)$ \\
\hline$C(6)-C(7)$ & $1.491(3)$ & $C(19)-C(21)$ & $1.550(3)$ \\
\hline$C(7)-C(12)$ & $1.393(3)$ & $\mathrm{C}(19)-\mathrm{P}(1)$ & $1.858(2)$ \\
\hline$C(7)-C(8)$ & $1.419(3)$ & $\mathrm{C}(20)-\mathrm{H}(20 \mathrm{~A})$ & 0.9600 \\
\hline $\mathrm{C}(8)-\mathrm{C}(9)$ & $1.368(3)$ & $\mathrm{C}(20)-\mathrm{H}(20 \mathrm{~B})$ & 0.9600 \\
\hline $\mathrm{C}(8)-\mathrm{H}(8)$ & 0.9300 & $\mathrm{C}(20)-\mathrm{H}(20 \mathrm{C})$ & 0.9600 \\
\hline$C(9)-C(10)$ & $1.399(3)$ & $\mathrm{C}(21)-\mathrm{H}(21 \mathrm{~A})$ & 0.9600 \\
\hline $\mathrm{C}(10)-\mathrm{C}(11)$ & $1.412(3)$ & $\mathrm{C}(21)-\mathrm{H}(21 \mathrm{~B})$ & 0.9600 \\
\hline $\mathrm{C}(10)-\mathrm{C}(13)$ & $1.428(3)$ & $\mathrm{C}(21)-\mathrm{H}(21 \mathrm{C})$ & 0.9600 \\
\hline $\mathrm{C}(11)-\mathrm{C}(12)$ & $1.389(3)$ & $\mathrm{C}(22)-\mathrm{H}(22 \mathrm{~A})$ & 0.9600 \\
\hline $\mathrm{C}(11)-\mathrm{N}(1)$ & $1.414(3)$ & $\mathrm{C}(22)-\mathrm{H}(22 \mathrm{~B})$ & 0.9600 \\
\hline $\mathrm{C}(12)-\mathrm{H}(12)$ & 0.9300 & $\mathrm{C}(22)-\mathrm{H}(22 \mathrm{C})$ & 0.9600 \\
\hline $\mathrm{C}(13)-\mathrm{C}(14)$ & $1.352(3)$ & $C(23)-C(24)$ & $1.386(3)$ \\
\hline $\mathrm{C}(13)-\mathrm{H}(13)$ & 0.9300 & $\mathrm{C}(23)-\mathrm{C}(28)$ & $1.391(3)$ \\
\hline $\mathrm{C}(14)-\mathrm{N}(1)$ & $1.405(2)$ & $\mathrm{C}(23)-\mathrm{H}(23)$ & 0.9300 \\
\hline $\mathrm{C}(14)-\mathrm{H}(14)$ & 0.9300 & $C(24)-C(25)$ & $1.382(3)$ \\
\hline $\mathrm{C}(15)-\mathrm{C}(17)$ & $1.531(3)$ & $\mathrm{C}(24)-\mathrm{H}(24)$ & 0.9300 \\
\hline $\mathrm{C}(15)-\mathrm{C}(18)$ & $1.535(3)$ & $C(25)-C(26)$ & $1.368(3)$ \\
\hline
\end{tabular}

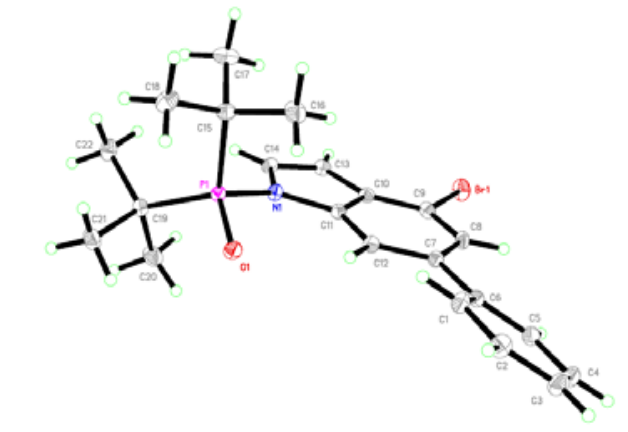

1.543(3)

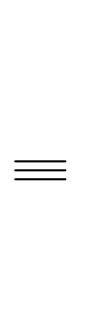




\begin{tabular}{|c|c|c|c|}
\hline $\mathrm{C}(25)-\mathrm{H}(25)$ & 0.9300 & $\mathrm{C}(42)-\mathrm{H}(42 \mathrm{~B})$ & 0.9600 \\
\hline$C(26)-C(27)$ & $1.382(3)$ & $\mathrm{C}(42)-\mathrm{H}(42 \mathrm{C})$ & 0.9600 \\
\hline $\mathrm{C}(26)-\mathrm{H}(26)$ & 0.9300 & $\mathrm{C}(43)-\mathrm{H}(43 \mathrm{~A})$ & 0.9600 \\
\hline $\mathrm{C}(27)-\mathrm{C}(28)$ & $1.400(3)$ & $\mathrm{C}(43)-\mathrm{H}(43 \mathrm{~B})$ & 0.9600 \\
\hline $\mathrm{C}(27)-\mathrm{H}(27)$ & 0.9300 & $\mathrm{C}(43)-\mathrm{H}(43 \mathrm{C})$ & 0.9600 \\
\hline $\mathrm{C}(28)-\mathrm{C}(29)$ & $1.495(3)$ & $\mathrm{C}(44)-\mathrm{H}(44 \mathrm{~A})$ & 0.9600 \\
\hline$C(29)-C(30)$ & $1.392(3)$ & $\mathrm{C}(44)-\mathrm{H}(44 \mathrm{~B})$ & 0.9600 \\
\hline$C(29)-C(34)$ & $1.416(3)$ & $\mathrm{C}(44)-\mathrm{H}(44 \mathrm{C})$ & 0.9600 \\
\hline$C(30)-C(31)$ & $1.391(3)$ & $\mathrm{N}(1)-\mathrm{P}(1)$ & $1.7150(17)$ \\
\hline $\mathrm{C}(30)-\mathrm{H}(30)$ & 0.9300 & $\mathrm{~N}(2)-\mathrm{P}(2)$ & $1.7176(16)$ \\
\hline $\mathrm{C}(31)-\mathrm{C}(32)$ & $1.412(3)$ & $\mathrm{O}(1)-\mathrm{P}(1)$ & $1.4830(14)$ \\
\hline$C(31)-N(2)$ & $1.412(2)$ & $\mathrm{O}(2)-\mathrm{P}(2)$ & $1.4837(14)$ \\
\hline$C(32)-C(33)$ & $1.395(3)$ & & \\
\hline$C(32)-C(35)$ & $1.430(3)$ & $C(2)-C(1)-C(6)$ & $121.7(2)$ \\
\hline$C(33)-C(34)$ & $1.371(3)$ & $\mathrm{C}(2)-\mathrm{C}(1)-\mathrm{H}(1)$ & 119.2 \\
\hline $\mathrm{C}(34)-\mathrm{H}(34)$ & 0.9300 & $\mathrm{C}(6)-\mathrm{C}(1)-\mathrm{H}(1)$ & 119.2 \\
\hline$C(35)-C(36)$ & $1.349(3)$ & $\mathrm{C}(1)-\mathrm{C}(2)-\mathrm{C}(3)$ & $120.2(2)$ \\
\hline $\mathrm{C}(35)-\mathrm{H}(35)$ & 0.9300 & $\mathrm{C}(1)-\mathrm{C}(2)-\mathrm{H}(2)$ & 119.9 \\
\hline$C(36)-N(2)$ & $1.396(3)$ & $\mathrm{C}(3)-\mathrm{C}(2)-\mathrm{H}(2)$ & 119.9 \\
\hline $\mathrm{C}(36)-\mathrm{H}(36)$ & 0.9300 & $C(4)-C(3)-C(2)$ & $118.9(2)$ \\
\hline C(37)-C(39) & $1.534(3)$ & $\mathrm{C}(4)-\mathrm{C}(3)-\mathrm{H}(3)$ & 120.5 \\
\hline $\mathrm{C}(37)-\mathrm{C}(38)$ & $1.536(3)$ & $\mathrm{C}(2)-\mathrm{C}(3)-\mathrm{H}(3)$ & 120.5 \\
\hline $\mathrm{C}(37)-\mathrm{C}(40)$ & $1.546(3)$ & $\mathrm{C}(5)-\mathrm{C}(4)-\mathrm{C}(3)$ & $121.2(2)$ \\
\hline $\mathrm{C}(37)-\mathrm{P}(2)$ & $1.849(2)$ & $\mathrm{C}(5)-\mathrm{C}(4)-\mathrm{H}(4)$ & 119.4 \\
\hline $\mathrm{C}(38)-\mathrm{H}(38 \mathrm{~A})$ & 0.9600 & $\mathrm{C}(3)-\mathrm{C}(4)-\mathrm{H}(4)$ & 119.4 \\
\hline $\mathrm{C}(38)-\mathrm{H}(38 \mathrm{~B})$ & 0.9600 & $C(4)-C(5)-C(6)$ & $120.8(2)$ \\
\hline $\mathrm{C}(38)-\mathrm{H}(38 \mathrm{C})$ & 0.9600 & $\mathrm{C}(4)-\mathrm{C}(5)-\mathrm{H}(5)$ & 119.6 \\
\hline $\mathrm{C}(39)-\mathrm{H}(39 \mathrm{~A})$ & 0.9600 & $\mathrm{C}(6)-\mathrm{C}(5)-\mathrm{H}(5)$ & 119.6 \\
\hline $\mathrm{C}(39)-\mathrm{H}(39 \mathrm{~B})$ & 0.9600 & $C(1)-C(6)-C(5)$ & $117.16(19)$ \\
\hline $\mathrm{C}(39)-\mathrm{H}(39 \mathrm{C})$ & 0.9600 & $\mathrm{C}(1)-\mathrm{C}(6)-\mathrm{C}(7)$ & $121.76(18)$ \\
\hline $\mathrm{C}(40)-\mathrm{H}(40 \mathrm{~A})$ & 0.9600 & $C(5)-C(6)-C(7)$ & $121.05(19)$ \\
\hline $\mathrm{C}(40)-\mathrm{H}(40 \mathrm{~B})$ & 0.9600 & $C(12)-C(7)-C(8)$ & $119.20(19)$ \\
\hline $\mathrm{C}(40)-\mathrm{H}(40 \mathrm{C})$ & 0.9600 & $C(12)-C(7)-C(6)$ & $120.11(18)$ \\
\hline$C(41)-C(42)$ & $1.532(3)$ & $C(8)-C(7)-C(6)$ & $120.66(18)$ \\
\hline$C(41)-C(43)$ & $1.541(3)$ & $C(9)-C(8)-C(7)$ & $120.63(19)$ \\
\hline$C(41)-C(44)$ & $1.548(3)$ & $\mathrm{C}(9)-\mathrm{C}(8)-\mathrm{H}(8)$ & 119.7 \\
\hline $\mathrm{C}(41)-\mathrm{P}(2)$ & $1.839(2)$ & $\mathrm{C}(7)-\mathrm{C}(8)-\mathrm{H}(8)$ & 119.7 \\
\hline $\mathrm{C}(42)-\mathrm{H}(42 \mathrm{~A})$ & 0.9600 & $\mathrm{C}(8)-\mathrm{C}(9)-\mathrm{C}(10)$ & $121.56(19)$ \\
\hline
\end{tabular}




\begin{tabular}{|c|c|c|c|}
\hline $\mathrm{C}(8)-\mathrm{C}(9)-\mathrm{Br}(1)$ & $119.80(16)$ & $\mathrm{C}(15)-\mathrm{C}(18)-\mathrm{H}(18 \mathrm{C})$ & 109.5 \\
\hline$C(10)-C(9)-B r(1)$ & $118.64(15)$ & $\mathrm{H}(18 \mathrm{~A})-\mathrm{C}(18)-\mathrm{H}(18 \mathrm{C})$ & 109.5 \\
\hline $\mathrm{C}(9)-\mathrm{C}(10)-\mathrm{C}(11)$ & $117.07(18)$ & $\mathrm{H}(18 \mathrm{~B})-\mathrm{C}(18)-\mathrm{H}(18 \mathrm{C})$ & 109.5 \\
\hline C(9)-C(10)-C(13) & $135.23(19)$ & $C(22)-C(19)-C(20)$ & $109.58(18)$ \\
\hline$C(11)-C(10)-C(13)$ & $107.69(18)$ & $C(22)-C(19)-C(21)$ & $108.61(18)$ \\
\hline $\mathrm{C}(12)-\mathrm{C}(11)-\mathrm{C}(10)$ & $122.49(19)$ & $C(20)-C(19)-C(21)$ & $107.47(18)$ \\
\hline $\mathrm{C}(12)-\mathrm{C}(11)-\mathrm{N}(1)$ & $130.07(18)$ & $\mathrm{C}(22)-\mathrm{C}(19)-\mathrm{P}(1)$ & $117.24(15)$ \\
\hline $\mathrm{C}(10)-\mathrm{C}(11)-\mathrm{N}(1)$ & $107.40(17)$ & $\mathrm{C}(20)-\mathrm{C}(19)-\mathrm{P}(1)$ & $108.10(15)$ \\
\hline$C(11)-C(12)-C(7)$ & 119.03(19) & $\mathrm{C}(21)-\mathrm{C}(19)-\mathrm{P}(1)$ & $105.38(14)$ \\
\hline $\mathrm{C}(11)-\mathrm{C}(12)-\mathrm{H}(12)$ & 120.5 & $\mathrm{C}(19)-\mathrm{C}(20)-\mathrm{H}(20 \mathrm{~A})$ & 109.5 \\
\hline $\mathrm{C}(7)-\mathrm{C}(12)-\mathrm{H}(12)$ & 120.5 & $\mathrm{C}(19)-\mathrm{C}(20)-\mathrm{H}(20 \mathrm{~B})$ & 109.5 \\
\hline $\mathrm{C}(14)-\mathrm{C}(13)-\mathrm{C}(10)$ & $107.40(18)$ & $\mathrm{H}(20 \mathrm{~A})-\mathrm{C}(20)-\mathrm{H}(20 \mathrm{~B})$ & 109.5 \\
\hline $\mathrm{C}(14)-\mathrm{C}(13)-\mathrm{H}(13)$ & 126.3 & $\mathrm{C}(19)-\mathrm{C}(20)-\mathrm{H}(20 \mathrm{C})$ & 109.5 \\
\hline $\mathrm{C}(10)-\mathrm{C}(13)-\mathrm{H}(13)$ & 126.3 & $\mathrm{H}(20 \mathrm{~A})-\mathrm{C}(20)-\mathrm{H}(20 \mathrm{C})$ & 109.5 \\
\hline $\mathrm{C}(13)-\mathrm{C}(14)-\mathrm{N}(1)$ & $110.77(18)$ & $\mathrm{H}(20 \mathrm{~B})-\mathrm{C}(20)-\mathrm{H}(20 \mathrm{C})$ & 109.5 \\
\hline $\mathrm{C}(13)-\mathrm{C}(14)-\mathrm{H}(14)$ & 124.6 & $\mathrm{C}(19)-\mathrm{C}(21)-\mathrm{H}(21 \mathrm{~A})$ & 109.5 \\
\hline $\mathrm{N}(1)-\mathrm{C}(14)-\mathrm{H}(14)$ & 124.6 & $\mathrm{C}(19)-\mathrm{C}(21)-\mathrm{H}(21 \mathrm{~B})$ & 109.5 \\
\hline $\mathrm{C}(17)-\mathrm{C}(15)-\mathrm{C}(18)$ & $110.54(18)$ & $\mathrm{H}(21 \mathrm{~A})-\mathrm{C}(21)-\mathrm{H}(21 \mathrm{~B})$ & 109.5 \\
\hline$C(17)-C(15)-C(16)$ & 109.18(19) & $\mathrm{C}(19)-\mathrm{C}(21)-\mathrm{H}(21 \mathrm{C})$ & 109.5 \\
\hline $\mathrm{C}(18)-\mathrm{C}(15)-\mathrm{C}(16)$ & $108.20(18)$ & $\mathrm{H}(21 \mathrm{~A})-\mathrm{C}(21)-\mathrm{H}(21 \mathrm{C})$ & 109.5 \\
\hline$C(17)-C(15)-P(1)$ & $113.28(14)$ & $\mathrm{H}(21 \mathrm{~B})-\mathrm{C}(21)-\mathrm{H}(21 \mathrm{C})$ & 109.5 \\
\hline C(18)-C(15)-P(1) & $109.36(15)$ & $\mathrm{C}(19)-\mathrm{C}(22)-\mathrm{H}(22 \mathrm{~A})$ & 109.5 \\
\hline$C(16)-C(15)-P(1)$ & $106.07(14)$ & $\mathrm{C}(19)-\mathrm{C}(22)-\mathrm{H}(22 \mathrm{~B})$ & 109.5 \\
\hline $\mathrm{C}(15)-\mathrm{C}(16)-\mathrm{H}(16 \mathrm{~A})$ & 109.5 & $\mathrm{H}(22 \mathrm{~A})-\mathrm{C}(22)-\mathrm{H}(22 \mathrm{~B})$ & 109.5 \\
\hline $\mathrm{C}(15)-\mathrm{C}(16)-\mathrm{H}(16 \mathrm{~B})$ & 109.5 & $\mathrm{C}(19)-\mathrm{C}(22)-\mathrm{H}(22 \mathrm{C})$ & 109.5 \\
\hline $\mathrm{H}(16 \mathrm{~A})-\mathrm{C}(16)-\mathrm{H}(16 \mathrm{~B})$ & 109.5 & $\mathrm{H}(22 \mathrm{~A})-\mathrm{C}(22)-\mathrm{H}(22 \mathrm{C})$ & 109.5 \\
\hline $\mathrm{C}(15)-\mathrm{C}(16)-\mathrm{H}(16 \mathrm{C})$ & 109.5 & $\mathrm{H}(22 \mathrm{~B})-\mathrm{C}(22)-\mathrm{H}(22 \mathrm{C})$ & 109.5 \\
\hline $\mathrm{H}(16 \mathrm{~A})-\mathrm{C}(16)-\mathrm{H}(16 \mathrm{C})$ & 109.5 & $\mathrm{C}(24)-\mathrm{C}(23)-\mathrm{C}(28)$ & $121.8(2)$ \\
\hline $\mathrm{H}(16 \mathrm{~B})-\mathrm{C}(16)-\mathrm{H}(16 \mathrm{C})$ & 109.5 & $\mathrm{C}(24)-\mathrm{C}(23)-\mathrm{H}(23)$ & 119.1 \\
\hline $\mathrm{C}(15)-\mathrm{C}(17)-\mathrm{H}(17 \mathrm{~A})$ & 109.5 & $\mathrm{C}(28)-\mathrm{C}(23)-\mathrm{H}(23)$ & 119.1 \\
\hline $\mathrm{C}(15)-\mathrm{C}(17)-\mathrm{H}(17 \mathrm{~B})$ & 109.5 & $\mathrm{C}(25)-\mathrm{C}(24)-\mathrm{C}(23)$ & $120.1(2)$ \\
\hline $\mathrm{H}(17 \mathrm{~A})-\mathrm{C}(17)-\mathrm{H}(17 \mathrm{~B})$ & 109.5 & $\mathrm{C}(25)-\mathrm{C}(24)-\mathrm{H}(24)$ & 120.0 \\
\hline $\mathrm{C}(15)-\mathrm{C}(17)-\mathrm{H}(17 \mathrm{C})$ & 109.5 & $\mathrm{C}(23)-\mathrm{C}(24)-\mathrm{H}(24)$ & 120.0 \\
\hline $\mathrm{H}(17 \mathrm{~A})-\mathrm{C}(17)-\mathrm{H}(17 \mathrm{C})$ & 109.5 & $C(26)-C(25)-C(24)$ & $119.2(2)$ \\
\hline $\mathrm{H}(17 \mathrm{~B})-\mathrm{C}(17)-\mathrm{H}(17 \mathrm{C})$ & 109.5 & $\mathrm{C}(26)-\mathrm{C}(25)-\mathrm{H}(25)$ & 120.4 \\
\hline $\mathrm{C}(15)-\mathrm{C}(18)-\mathrm{H}(18 \mathrm{~A})$ & 109.5 & $\mathrm{C}(24)-\mathrm{C}(25)-\mathrm{H}(25)$ & 120.4 \\
\hline $\mathrm{C}(15)-\mathrm{C}(18)-\mathrm{H}(18 \mathrm{~B})$ & 109.5 & $C(25)-C(26)-C(27)$ & $120.7(2)$ \\
\hline $\mathrm{H}(18 \mathrm{~A})-\mathrm{C}(18)-\mathrm{H}(18 \mathrm{~B})$ & 109.5 & $\mathrm{C}(25)-\mathrm{C}(26)-\mathrm{H}(26)$ & 119.6 \\
\hline
\end{tabular}




\begin{tabular}{|c|c|c|c|}
\hline $\mathrm{C}(27)-\mathrm{C}(26)-\mathrm{H}(26)$ & 119.6 & $\mathrm{C}(37)-\mathrm{C}(38)-\mathrm{H}(38 \mathrm{~B})$ & 109.5 \\
\hline$C(26)-C(27)-C(28)$ & $121.5(2)$ & $\mathrm{H}(38 \mathrm{~A})-\mathrm{C}(38)-\mathrm{H}(38 \mathrm{~B})$ & 109.5 \\
\hline $\mathrm{C}(26)-\mathrm{C}(27)-\mathrm{H}(27)$ & 119.3 & $\mathrm{C}(37)-\mathrm{C}(38)-\mathrm{H}(38 \mathrm{C})$ & 109.5 \\
\hline $\mathrm{C}(28)-\mathrm{C}(27)-\mathrm{H}(27)$ & 119.3 & $\mathrm{H}(38 \mathrm{~A})-\mathrm{C}(38)-\mathrm{H}(38 \mathrm{C})$ & 109.5 \\
\hline$C(23)-C(28)-C(27)$ & $116.6(2)$ & $\mathrm{H}(38 \mathrm{~B})-\mathrm{C}(38)-\mathrm{H}(38 \mathrm{C})$ & 109.5 \\
\hline$C(23)-C(28)-C(29)$ & $121.73(18)$ & $\mathrm{C}(37)-\mathrm{C}(39)-\mathrm{H}(39 \mathrm{~A})$ & 109.5 \\
\hline$C(27)-C(28)-C(29)$ & $121.65(19)$ & $\mathrm{C}(37)-\mathrm{C}(39)-\mathrm{H}(39 \mathrm{~B})$ & 109.5 \\
\hline$C(30)-C(29)-C(34)$ & $119.22(19)$ & $\mathrm{H}(39 \mathrm{~A})-\mathrm{C}(39)-\mathrm{H}(39 \mathrm{~B})$ & 109.5 \\
\hline$C(30)-C(29)-C(28)$ & $120.48(18)$ & $\mathrm{C}(37)-\mathrm{C}(39)-\mathrm{H}(39 \mathrm{C})$ & 109.5 \\
\hline$C(34)-C(29)-C(28)$ & $120.30(18)$ & $\mathrm{H}(39 \mathrm{~A})-\mathrm{C}(39)-\mathrm{H}(39 \mathrm{C})$ & 109.5 \\
\hline$C(31)-C(30)-C(29)$ & $118.73(18)$ & H(39B)-C(39)-H(39C) & 109.5 \\
\hline $\mathrm{C}(31)-\mathrm{C}(30)-\mathrm{H}(30)$ & 120.6 & $\mathrm{C}(37)-\mathrm{C}(40)-\mathrm{H}(40 \mathrm{~A})$ & 109.5 \\
\hline $\mathrm{C}(29)-\mathrm{C}(30)-\mathrm{H}(30)$ & 120.6 & $\mathrm{C}(37)-\mathrm{C}(40)-\mathrm{H}(40 \mathrm{~B})$ & 109.5 \\
\hline$C(30)-C(31)-C(32)$ & $122.82(18)$ & $\mathrm{H}(40 \mathrm{~A})-\mathrm{C}(40)-\mathrm{H}(40 \mathrm{~B})$ & 109.5 \\
\hline $\mathrm{C}(30)-\mathrm{C}(31)-\mathrm{N}(2)$ & $130.22(17)$ & $C(37)-C(40)-H(40 C)$ & 109.5 \\
\hline $\mathrm{C}(32)-\mathrm{C}(31)-\mathrm{N}(2)$ & $106.96(17)$ & $\mathrm{H}(40 \mathrm{~A})-\mathrm{C}(40)-\mathrm{H}(40 \mathrm{C})$ & 109.5 \\
\hline$C(33)-C(32)-C(31)$ & $116.90(19)$ & $\mathrm{H}(40 \mathrm{~B})-\mathrm{C}(40)-\mathrm{H}(40 \mathrm{C})$ & 109.5 \\
\hline$C(33)-C(32)-C(35)$ & $135.26(19)$ & $C(42)-C(41)-C(43)$ & $110.16(18)$ \\
\hline$C(31)-C(32)-C(35)$ & $107.84(17)$ & $C(42)-C(41)-C(44)$ & $109.01(19)$ \\
\hline$C(34)-C(33)-C(32)$ & $121.53(18)$ & $C(43)-C(41)-C(44)$ & $108.20(19)$ \\
\hline$C(34)-C(33)-B r(2)$ & $119.72(15)$ & $\mathrm{C}(42)-\mathrm{C}(41)-\mathrm{P}(2)$ & $114.07(15)$ \\
\hline$C(32)-C(33)-B r(2)$ & $118.74(16)$ & $\mathrm{C}(43)-\mathrm{C}(41)-\mathrm{P}(2)$ & $109.73(15)$ \\
\hline$C(33)-C(34)-C(29)$ & $120.79(18)$ & $\mathrm{C}(44)-\mathrm{C}(41)-\mathrm{P}(2)$ & $105.43(15)$ \\
\hline $\mathrm{C}(33)-\mathrm{C}(34)-\mathrm{H}(34)$ & 119.6 & $\mathrm{C}(41)-\mathrm{C}(42)-\mathrm{H}(42 \mathrm{~A})$ & 109.5 \\
\hline $\mathrm{C}(29)-\mathrm{C}(34)-\mathrm{H}(34)$ & 119.6 & $\mathrm{C}(41)-\mathrm{C}(42)-\mathrm{H}(42 \mathrm{~B})$ & 109.5 \\
\hline$C(36)-C(35)-C(32)$ & $107.09(18)$ & $\mathrm{H}(42 \mathrm{~A})-\mathrm{C}(42)-\mathrm{H}(42 \mathrm{~B})$ & 109.5 \\
\hline $\mathrm{C}(36)-\mathrm{C}(35)-\mathrm{H}(35)$ & 126.5 & $\mathrm{C}(41)-\mathrm{C}(42)-\mathrm{H}(42 \mathrm{C})$ & 109.5 \\
\hline $\mathrm{C}(32)-\mathrm{C}(35)-\mathrm{H}(35)$ & 126.5 & $\mathrm{H}(42 \mathrm{~A})-\mathrm{C}(42)-\mathrm{H}(42 \mathrm{C})$ & 109.5 \\
\hline $\mathrm{C}(35)-\mathrm{C}(36)-\mathrm{N}(2)$ & $110.93(18)$ & $\mathrm{H}(42 \mathrm{~B})-\mathrm{C}(42)-\mathrm{H}(42 \mathrm{C})$ & 109.5 \\
\hline $\mathrm{C}(35)-\mathrm{C}(36)-\mathrm{H}(36)$ & 124.5 & $\mathrm{C}(41)-\mathrm{C}(43)-\mathrm{H}(43 \mathrm{~A})$ & 109.5 \\
\hline $\mathrm{N}(2)-\mathrm{C}(36)-\mathrm{H}(36)$ & 124.5 & $\mathrm{C}(41)-\mathrm{C}(43)-\mathrm{H}(43 \mathrm{~B})$ & 109.5 \\
\hline $\mathrm{C}(39)-\mathrm{C}(37)-\mathrm{C}(38)$ & $109.8(2)$ & $\mathrm{H}(43 \mathrm{~A})-\mathrm{C}(43)-\mathrm{H}(43 \mathrm{~B})$ & 109.5 \\
\hline$C(39)-C(37)-C(40)$ & $108.67(18)$ & $\mathrm{C}(41)-\mathrm{C}(43)-\mathrm{H}(43 \mathrm{C})$ & 109.5 \\
\hline$C(38)-C(37)-C(40)$ & $107.84(19)$ & $\mathrm{H}(43 \mathrm{~A})-\mathrm{C}(43)-\mathrm{H}(43 \mathrm{C})$ & 109.5 \\
\hline C(39)-C(37)-P(2) & $116.95(16)$ & $\mathrm{H}(43 \mathrm{~B})-\mathrm{C}(43)-\mathrm{H}(43 \mathrm{C})$ & 109.5 \\
\hline $\mathrm{C}(38)-\mathrm{C}(37)-\mathrm{P}(2)$ & $107.51(15)$ & $\mathrm{C}(41)-\mathrm{C}(44)-\mathrm{H}(44 \mathrm{~A})$ & 109.5 \\
\hline $\mathrm{C}(40)-\mathrm{C}(37)-\mathrm{P}(2)$ & $105.66(14)$ & $\mathrm{C}(41)-\mathrm{C}(44)-\mathrm{H}(44 \mathrm{~B})$ & 109.5 \\
\hline $\mathrm{C}(37)-\mathrm{C}(38)-\mathrm{H}(38 \mathrm{~A})$ & 109.5 & $\mathrm{H}(44 \mathrm{~A})-\mathrm{C}(44)-\mathrm{H}(44 \mathrm{~B})$ & 109.5 \\
\hline
\end{tabular}




$\begin{array}{llll}\mathrm{C}(41)-\mathrm{C}(44)-\mathrm{H}(44 \mathrm{C}) & 109.5 & \mathrm{~N}(1)-\mathrm{P}(1)-\mathrm{C}(15) & 104.78(9) \\ \mathrm{H}(44 \mathrm{~A})-\mathrm{C}(44)-\mathrm{H}(44 \mathrm{C}) & 109.5 & \mathrm{O}(1)-\mathrm{P}(1)-\mathrm{C}(19) & 109.77(9) \\ \mathrm{H}(44 \mathrm{~B})-\mathrm{C}(44)-\mathrm{H}(44 \mathrm{C}) & 109.5 & \mathrm{~N}(1)-\mathrm{P}(1)-\mathrm{C}(19) & 107.38(9) \\ \mathrm{C}(14)-\mathrm{N}(1)-\mathrm{C}(11) & 106.74(16) & \mathrm{C}(15)-\mathrm{P}(1)-\mathrm{C}(19) & 114.84(10) \\ \mathrm{C}(14)-\mathrm{N}(1)-\mathrm{P}(1) & 128.05(14) & \mathrm{O}(2)-\mathrm{P}(2)-\mathrm{N}(2) & 109.15(8) \\ \mathrm{C}(11)-\mathrm{N}(1)-\mathrm{P}(1) & 124.63(13) & \mathrm{O}(2)-\mathrm{P}(2)-\mathrm{C}(41) & 110.94(9) \\ \mathrm{C}(36)-\mathrm{N}(2)-\mathrm{C}(31) & 107.18(15) & \mathrm{N}(2)-\mathrm{P}(2)-\mathrm{C}(41) & 104.28(9) \\ \mathrm{C}(36)-\mathrm{N}(2)-\mathrm{P}(2) & 127.06(14) & \mathrm{O}(2)-\mathrm{P}(2)-\mathrm{C}(37) & 110.33(9) \\ \mathrm{C}(31)-\mathrm{N}(2)-\mathrm{P}(2) & 125.56(13) & \mathrm{N}(2)-\mathrm{P}(2)-\mathrm{C}(37) & 106.78(9) \\ \mathrm{O}(1)-\mathrm{P}(1)-\mathrm{N}(1) & 108.74(8) & \mathrm{C}(41)-\mathrm{P}(2)-\mathrm{C}(37) & 114.97(10) \\ \mathrm{O}(1)-\mathrm{P}(1)-\mathrm{C}(15) & 111.01(9) & & \end{array}$<smiles></smiles>

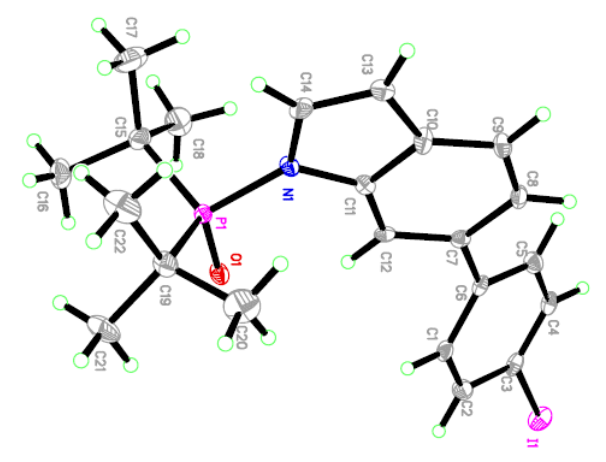

$\begin{array}{llll}\mathrm{C}(1)-\mathrm{C}(6) & 1.390(15) & \mathrm{C}(10)-\mathrm{C}(11) & 1.402(14) \\ \mathrm{C}(1)-\mathrm{C}(2) & 1.393(15) & \mathrm{C}(10)-\mathrm{C}(13) & 1.443(14) \\ \mathrm{C}(1)-\mathrm{H}(1) & 0.9300 & \mathrm{C}(11)-\mathrm{C}(12) & 1.394(14) \\ \mathrm{C}(2)-\mathrm{C}(3) & 1.386(15) & \mathrm{C}(11)-\mathrm{N}(1) & 1.412(13) \\ \mathrm{C}(2)-\mathrm{H}(2) & 0.9300 & \mathrm{C}(12)-\mathrm{H}(12) & 0.9300 \\ \mathrm{C}(3)-\mathrm{C}(4) & 1.363(16) & \mathrm{C}(13)-\mathrm{C}(14) & 1.353(15) \\ \mathrm{C}(3)-\mathrm{I}(1) & 2.100(10) & \mathrm{C}(13)-\mathrm{H}(13) & 0.9300 \\ \mathrm{C}(4)-\mathrm{C}(5) & 1.399(15) & \mathrm{C}(14)-\mathrm{N}(1) & 1.400(13) \\ \mathrm{C}(4)-\mathrm{H}(4) & 0.9300 & \mathrm{C}(14)-\mathrm{H}(14) & 0.9300 \\ \mathrm{C}(5)-\mathrm{C}(6) & 1.394(15) & \mathrm{C}(15)-\mathrm{C}(16) & 1.524(16) \\ \mathrm{C}(5)-\mathrm{H}(5) & 0.9300 & \mathrm{C}(15)-\mathrm{C}(17) & 1.537(17) \\ \mathrm{C}(6)-\mathrm{C}(7) & 1.488(14) & \mathrm{C}(15)-\mathrm{C}(18) & 1.555(18) \\ \mathrm{C}(7)-\mathrm{C}(12) & 1.393(14) & \mathrm{C}(15)-\mathrm{P}(1) & 1.837(11) \\ \mathrm{C}(7)-\mathrm{C}(8) & 1.410(14) & \mathrm{C}(16)-\mathrm{H}(16 \mathrm{~A}) & 0.9600 \\ \mathrm{C}(8)-\mathrm{C}(9) & 1.398(16) & \mathrm{C}(16)-\mathrm{H}(16 \mathrm{~B}) & 0.9600 \\ \mathrm{C}(8)-\mathrm{H}(8) & 0.9300 & \mathrm{C}(16)-\mathrm{H}(16 \mathrm{C}) & 0.9600 \\ \mathrm{C}(9)-\mathrm{C}(10) & 1.397(15) & \mathrm{C}(17)-\mathrm{H}(17 \mathrm{~A}) & 0.9600 \\ \mathrm{C}(9)-\mathrm{H}(9) & 0.9300 & \mathrm{C}(17)-\mathrm{H}(17 \mathrm{~B}) & 0.9600\end{array}$




\begin{tabular}{|c|c|c|c|}
\hline $\mathrm{C}(17)-\mathrm{H}(17 \mathrm{C})$ & 0.9600 & $\mathrm{C}(34)-\mathrm{C}(35)$ & $1.394(14)$ \\
\hline $\mathrm{C}(18)-\mathrm{H}(18 \mathrm{~A})$ & 0.9600 & $\mathrm{C}(34)-\mathrm{H}(34)$ & 0.9300 \\
\hline $\mathrm{C}(18)-\mathrm{H}(18 \mathrm{~B})$ & 0.9600 & $C(36)-C(37)$ & $1.339(14)$ \\
\hline $\mathrm{C}(18)-\mathrm{H}(18 \mathrm{C})$ & 0.9600 & $C(36)-N(2)$ & $1.403(12)$ \\
\hline$C(19)-C(20)$ & $1.522(18)$ & $\mathrm{C}(36)-\mathrm{H}(36)$ & 0.9300 \\
\hline$C(19)-C(22)$ & $1.528(16)$ & $\mathrm{C}(37)-\mathrm{H}(37)$ & 0.9300 \\
\hline C(19)-C(21) & $1.538(16)$ & $\mathrm{C}(38)-\mathrm{C}(45)$ & $1.536(15)$ \\
\hline C(19)-P(1) & $1.855(11)$ & $\mathrm{C}(38)-\mathrm{H}(38 \mathrm{~A})$ & 0.9600 \\
\hline $\mathrm{C}(20)-\mathrm{H}(20 \mathrm{~A})$ & 0.9600 & $\mathrm{C}(38)-\mathrm{H}(38 \mathrm{~B})$ & 0.9600 \\
\hline $\mathrm{C}(20)-\mathrm{H}(20 \mathrm{~B})$ & 0.9600 & $\mathrm{C}(38)-\mathrm{H}(38 \mathrm{C})$ & 0.9600 \\
\hline $\mathrm{C}(20)-\mathrm{H}(20 \mathrm{C})$ & 0.9600 & $C(39)-C(45)$ & $1.540(14)$ \\
\hline $\mathrm{C}(21)-\mathrm{H}(21 \mathrm{~A})$ & 0.9600 & $\mathrm{C}(39)-\mathrm{H}(39 \mathrm{~A})$ & 0.9600 \\
\hline $\mathrm{C}(21)-\mathrm{H}(21 \mathrm{~B})$ & 0.9600 & C(39)-H(39B) & 0.9600 \\
\hline $\mathrm{C}(21)-\mathrm{H}(21 \mathrm{C})$ & 0.9600 & $\mathrm{C}(39)-\mathrm{H}(39 \mathrm{C})$ & 0.9600 \\
\hline $\mathrm{C}(22)-\mathrm{H}(22 \mathrm{~A})$ & 0.9600 & $C(40)-C(45)$ & $1.552(14)$ \\
\hline $\mathrm{C}(22)-\mathrm{H}(22 \mathrm{~B})$ & 0.9600 & $\mathrm{C}(40)-\mathrm{H}(40 \mathrm{~A})$ & 0.9600 \\
\hline $\mathrm{C}(22)-\mathrm{H}(22 \mathrm{C})$ & 0.9600 & $\mathrm{C}(40)-\mathrm{H}(40 \mathrm{~B})$ & 0.9600 \\
\hline$C(24)-C(29)$ & $1.377(15)$ & $\mathrm{C}(40)-\mathrm{H}(40 \mathrm{C})$ & 0.9600 \\
\hline$C(24)-C(25)$ & $1.383(15)$ & $C(41)-C(44)$ & $1.539(16)$ \\
\hline $\mathrm{C}(24)-\mathrm{H}(24)$ & 0.9300 & $C(41)-C(42)$ & $1.541(16)$ \\
\hline$C(25)-C(26)$ & $1.369(16)$ & $C(41)-C(43)$ & $1.548(14)$ \\
\hline C(25)-I(2) & $2.095(11)$ & $\mathrm{C}(41)-\mathrm{P}(2)$ & $1.853(10)$ \\
\hline$C(26)-C(27)$ & $1.386(15)$ & $\mathrm{C}(42)-\mathrm{H}(42 \mathrm{~A})$ & 0.9600 \\
\hline $\mathrm{C}(26)-\mathrm{H}(26)$ & 0.9300 & $\mathrm{C}(42)-\mathrm{H}(42 \mathrm{~B})$ & 0.9600 \\
\hline $\mathrm{C}(27)-\mathrm{C}(28)$ & $1.403(14)$ & $\mathrm{C}(42)-\mathrm{H}(42 \mathrm{C})$ & 0.9600 \\
\hline $\mathrm{C}(27)-\mathrm{H}(27)$ & 0.9300 & $\mathrm{C}(43)-\mathrm{H}(43 \mathrm{~A})$ & 0.9600 \\
\hline $\mathrm{C}(28)-\mathrm{C}(29)$ & $1.410(15)$ & $\mathrm{C}(43)-\mathrm{H}(43 \mathrm{~B})$ & 0.9600 \\
\hline $\mathrm{C}(28)-\mathrm{C}(35)$ & $1.467(14)$ & $\mathrm{C}(43)-\mathrm{H}(43 \mathrm{C})$ & 0.9600 \\
\hline C(29)-H(29) & 0.9300 & $\mathrm{C}(44)-\mathrm{H}(44 \mathrm{~A})$ & 0.9600 \\
\hline$C(30)-C(31)$ & $1.376(15)$ & $\mathrm{C}(44)-\mathrm{H}(44 \mathrm{~B})$ & 0.9600 \\
\hline$C(30)-C(35)$ & $1.420(14)$ & $\mathrm{C}(44)-\mathrm{H}(44 \mathrm{C})$ & 0.9600 \\
\hline $\mathrm{C}(30)-\mathrm{H}(30)$ & 0.9300 & $\mathrm{C}(45)-\mathrm{P}(2)$ & $1.841(10)$ \\
\hline $\mathrm{C}(31)-\mathrm{C}(32)$ & $1.394(15)$ & $\mathrm{N}(1)-\mathrm{P}(1)$ & $1.714(8)$ \\
\hline $\mathrm{C}(31)-\mathrm{H}(31)$ & 0.9300 & $\mathrm{~N}(2)-\mathrm{P}(2)$ & $1.720(8)$ \\
\hline$C(32)-C(33)$ & $1.405(13)$ & $\mathrm{O}(1)-\mathrm{P}(1)$ & $1.477(7)$ \\
\hline$C(32)-C(37)$ & $1.459(14)$ & $\mathrm{O}(2)-\mathrm{P}(2)$ & $1.472(7)$ \\
\hline$C(33)-C(34)$ & $1.394(14)$ & & \\
\hline $\mathrm{C}(33)-\mathrm{N}(2)$ & $1.409(12)$ & $C(6)-C(1)-C(2)$ & $121.5(10)$ \\
\hline
\end{tabular}




\begin{tabular}{|c|c|c|c|}
\hline $\mathrm{C}(6)-\mathrm{C}(1)-\mathrm{H}(1)$ & 119.3 & $\mathrm{C}(13)-\mathrm{C}(14)-\mathrm{N}(1)$ & 111.2(9) \\
\hline $\mathrm{C}(2)-\mathrm{C}(1)-\mathrm{H}(1)$ & 119.3 & $\mathrm{C}(13)-\mathrm{C}(14)-\mathrm{H}(14)$ & 124.4 \\
\hline$C(3)-C(2)-C(1)$ & $118.9(10)$ & $\mathrm{N}(1)-\mathrm{C}(14)-\mathrm{H}(14)$ & 124.4 \\
\hline $\mathrm{C}(3)-\mathrm{C}(2)-\mathrm{H}(2)$ & 120.6 & $C(16)-C(15)-C(17)$ & $110.7(11)$ \\
\hline $\mathrm{C}(1)-\mathrm{C}(2)-\mathrm{H}(2)$ & 120.6 & $C(16)-C(15)-C(18)$ & $108.0(10)$ \\
\hline$C(4)-C(3)-C(2)$ & $121.2(10)$ & $\mathrm{C}(17)-\mathrm{C}(15)-\mathrm{C}(18)$ & $108.2(11)$ \\
\hline $\mathrm{C}(4)-\mathrm{C}(3)-\mathrm{I}(1)$ & $118.9(8)$ & $\mathrm{C}(16)-\mathrm{C}(15)-\mathrm{P}(1)$ & $108.8(8)$ \\
\hline $\mathrm{C}(2)-\mathrm{C}(3)-\mathrm{I}(1)$ & 119.9(8) & $\mathrm{C}(17)-\mathrm{C}(15)-\mathrm{P}(1)$ & $116.2(9)$ \\
\hline$C(3)-C(4)-C(5)$ & $119.6(10)$ & $\mathrm{C}(18)-\mathrm{C}(15)-\mathrm{P}(1)$ & $104.5(8)$ \\
\hline $\mathrm{C}(3)-\mathrm{C}(4)-\mathrm{H}(4)$ & 120.2 & $\mathrm{C}(15)-\mathrm{C}(16)-\mathrm{H}(16 \mathrm{~A})$ & 109.5 \\
\hline $\mathrm{C}(5)-\mathrm{C}(4)-\mathrm{H}(4)$ & 120.2 & $\mathrm{C}(15)-\mathrm{C}(16)-\mathrm{H}(16 \mathrm{~B})$ & 109.5 \\
\hline$C(6)-C(5)-C(4)$ & $120.9(10)$ & $\mathrm{H}(16 \mathrm{~A})-\mathrm{C}(16)-\mathrm{H}(16 \mathrm{~B})$ & 109.5 \\
\hline $\mathrm{C}(6)-\mathrm{C}(5)-\mathrm{H}(5)$ & 119.5 & $\mathrm{C}(15)-\mathrm{C}(16)-\mathrm{H}(16 \mathrm{C})$ & 109.5 \\
\hline $\mathrm{C}(4)-\mathrm{C}(5)-\mathrm{H}(5)$ & 119.5 & $\mathrm{H}(16 \mathrm{~A})-\mathrm{C}(16)-\mathrm{H}(16 \mathrm{C})$ & 109.5 \\
\hline$C(1)-C(6)-C(5)$ & $118.0(10)$ & $\mathrm{H}(16 \mathrm{~B})-\mathrm{C}(16)-\mathrm{H}(16 \mathrm{C})$ & 109.5 \\
\hline$C(1)-C(6)-C(7)$ & $121.4(9)$ & $\mathrm{C}(15)-\mathrm{C}(17)-\mathrm{H}(17 \mathrm{~A})$ & 109.5 \\
\hline$C(5)-C(6)-C(7)$ & $120.6(10)$ & $\mathrm{C}(15)-\mathrm{C}(17)-\mathrm{H}(17 \mathrm{~B})$ & 109.5 \\
\hline $\mathrm{C}(12)-\mathrm{C}(7)-\mathrm{C}(8)$ & $120.0(9)$ & $\mathrm{H}(17 \mathrm{~A})-\mathrm{C}(17)-\mathrm{H}(17 \mathrm{~B})$ & 109.5 \\
\hline$C(12)-C(7)-C(6)$ & $120.8(9)$ & $\mathrm{C}(15)-\mathrm{C}(17)-\mathrm{H}(17 \mathrm{C})$ & 109.5 \\
\hline$C(8)-C(7)-C(6)$ & 119.2(9) & $\mathrm{H}(17 \mathrm{~A})-\mathrm{C}(17)-\mathrm{H}(17 \mathrm{C})$ & 109.5 \\
\hline $\mathrm{C}(9)-\mathrm{C}(8)-\mathrm{C}(7)$ & $120.9(10)$ & $\mathrm{H}(17 \mathrm{~B})-\mathrm{C}(17)-\mathrm{H}(17 \mathrm{C})$ & 109.5 \\
\hline $\mathrm{C}(9)-\mathrm{C}(8)-\mathrm{H}(8)$ & 119.5 & $\mathrm{C}(15)-\mathrm{C}(18)-\mathrm{H}(18 \mathrm{~A})$ & 109.5 \\
\hline $\mathrm{C}(7)-\mathrm{C}(8)-\mathrm{H}(8)$ & 119.5 & $\mathrm{C}(15)-\mathrm{C}(18)-\mathrm{H}(18 \mathrm{~B})$ & 109.5 \\
\hline $\mathrm{C}(10)-\mathrm{C}(9)-\mathrm{C}(8)$ & $118.8(10)$ & $\mathrm{H}(18 \mathrm{~A})-\mathrm{C}(18)-\mathrm{H}(18 \mathrm{~B})$ & 109.5 \\
\hline $\mathrm{C}(10)-\mathrm{C}(9)-\mathrm{H}(9)$ & 120.6 & $\mathrm{C}(15)-\mathrm{C}(18)-\mathrm{H}(18 \mathrm{C})$ & 109.5 \\
\hline $\mathrm{C}(8)-\mathrm{C}(9)-\mathrm{H}(9)$ & 120.6 & $\mathrm{H}(18 \mathrm{~A})-\mathrm{C}(18)-\mathrm{H}(18 \mathrm{C})$ & 109.5 \\
\hline C(9)-C(10)-C(11) & 119.9(9) & $\mathrm{H}(18 \mathrm{~B})-\mathrm{C}(18)-\mathrm{H}(18 \mathrm{C})$ & 109.5 \\
\hline $\mathrm{C}(9)-\mathrm{C}(10)-\mathrm{C}(13)$ & $132.6(10)$ & $C(20)-C(19)-C(22)$ & $109.3(12)$ \\
\hline $\mathrm{C}(11)-\mathrm{C}(10)-\mathrm{C}(13)$ & $107.3(9)$ & $C(20)-C(19)-C(21)$ & $110.1(11)$ \\
\hline$C(12)-C(11)-C(10)$ & $121.4(9)$ & $C(22)-C(19)-C(21)$ & $108.7(10)$ \\
\hline $\mathrm{C}(12)-\mathrm{C}(11)-\mathrm{N}(1)$ & $130.4(9)$ & C(20)-C(19)-P(1) & $105.5(8)$ \\
\hline $\mathrm{C}(10)-\mathrm{C}(11)-\mathrm{N}(1)$ & 108.1(8) & C(22)-C(19)-P(1) & $116.2(9)$ \\
\hline $\mathrm{C}(7)-\mathrm{C}(12)-\mathrm{C}(11)$ & $118.8(9)$ & C(21)-C(19)-P(1) & $107.0(8)$ \\
\hline $\mathrm{C}(7)-\mathrm{C}(12)-\mathrm{H}(12)$ & 120.6 & $\mathrm{C}(19)-\mathrm{C}(20)-\mathrm{H}(20 \mathrm{~A})$ & 109.5 \\
\hline $\mathrm{C}(11)-\mathrm{C}(12)-\mathrm{H}(12)$ & 120.6 & $\mathrm{C}(19)-\mathrm{C}(20)-\mathrm{H}(20 \mathrm{~B})$ & 109.5 \\
\hline$C(14)-C(13)-C(10)$ & $106.8(9)$ & $\mathrm{H}(20 \mathrm{~A})-\mathrm{C}(20)-\mathrm{H}(20 \mathrm{~B})$ & 109.5 \\
\hline $\mathrm{C}(14)-\mathrm{C}(13)-\mathrm{H}(13)$ & 126.6 & $\mathrm{C}(19)-\mathrm{C}(20)-\mathrm{H}(20 \mathrm{C})$ & 109.5 \\
\hline $\mathrm{C}(10)-\mathrm{C}(13)-\mathrm{H}(13)$ & 126.6 & $\mathrm{H}(20 \mathrm{~A})-\mathrm{C}(20)-\mathrm{H}(20 \mathrm{C})$ & 109.5 \\
\hline
\end{tabular}




\begin{tabular}{|c|c|c|c|}
\hline $\mathrm{H}(20 \mathrm{~B})-\mathrm{C}(20)-\mathrm{H}(20 \mathrm{C})$ & 109.5 & $C(31)-C(32)-C(37)$ & $133.6(9)$ \\
\hline $\mathrm{C}(19)-\mathrm{C}(21)-\mathrm{H}(21 \mathrm{~A})$ & 109.5 & $\mathrm{C}(33)-\mathrm{C}(32)-\mathrm{C}(37)$ & $106.6(8)$ \\
\hline $\mathrm{C}(19)-\mathrm{C}(21)-\mathrm{H}(21 \mathrm{~B})$ & 109.5 & $C(34)-C(33)-C(32)$ & $121.3(9)$ \\
\hline $\mathrm{H}(21 \mathrm{~A})-\mathrm{C}(21)-\mathrm{H}(21 \mathrm{~B})$ & 109.5 & $\mathrm{C}(34)-\mathrm{C}(33)-\mathrm{N}(2)$ & $130.6(9)$ \\
\hline $\mathrm{C}(19)-\mathrm{C}(21)-\mathrm{H}(21 \mathrm{C})$ & 109.5 & $\mathrm{C}(32)-\mathrm{C}(33)-\mathrm{N}(2)$ & $108.1(8)$ \\
\hline $\mathrm{H}(21 \mathrm{~A})-\mathrm{C}(21)-\mathrm{H}(21 \mathrm{C})$ & 109.5 & $\mathrm{C}(33)-\mathrm{C}(34)-\mathrm{C}(35)$ & $118.8(9)$ \\
\hline $\mathrm{H}(21 \mathrm{~B})-\mathrm{C}(21)-\mathrm{H}(21 \mathrm{C})$ & 109.5 & $\mathrm{C}(33)-\mathrm{C}(34)-\mathrm{H}(34)$ & 120.6 \\
\hline $\mathrm{C}(19)-\mathrm{C}(22)-\mathrm{H}(22 \mathrm{~A})$ & 109.5 & $\mathrm{C}(35)-\mathrm{C}(34)-\mathrm{H}(34)$ & 120.6 \\
\hline $\mathrm{C}(19)-\mathrm{C}(22)-\mathrm{H}(22 \mathrm{~B})$ & 109.5 & $C(34)-C(35)-C(30)$ & $119.4(9)$ \\
\hline $\mathrm{H}(22 \mathrm{~A})-\mathrm{C}(22)-\mathrm{H}(22 \mathrm{~B})$ & 109.5 & $\mathrm{C}(34)-\mathrm{C}(35)-\mathrm{C}(28)$ & 119.5(9) \\
\hline $\mathrm{C}(19)-\mathrm{C}(22)-\mathrm{H}(22 \mathrm{C})$ & 109.5 & $\mathrm{C}(30)-\mathrm{C}(35)-\mathrm{C}(28)$ & 121.1(9) \\
\hline $\mathrm{H}(22 \mathrm{~A})-\mathrm{C}(22)-\mathrm{H}(22 \mathrm{C})$ & 109.5 & $\mathrm{C}(37)-\mathrm{C}(36)-\mathrm{N}(2)$ & $110.9(9)$ \\
\hline $\mathrm{H}(22 \mathrm{~B})-\mathrm{C}(22)-\mathrm{H}(22 \mathrm{C})$ & 109.5 & $\mathrm{C}(37)-\mathrm{C}(36)-\mathrm{H}(36)$ & 124.6 \\
\hline$C(29)-C(24)-C(25)$ & $119.8(10)$ & $\mathrm{N}(2)-\mathrm{C}(36)-\mathrm{H}(36)$ & 124.6 \\
\hline $\mathrm{C}(29)-\mathrm{C}(24)-\mathrm{H}(24)$ & 120.1 & $C(36)-C(37)-C(32)$ & 107.4(9) \\
\hline $\mathrm{C}(25)-\mathrm{C}(24)-\mathrm{H}(24)$ & 120.1 & $\mathrm{C}(36)-\mathrm{C}(37)-\mathrm{H}(37)$ & 126.3 \\
\hline$C(26)-C(25)-C(24)$ & $119.8(10)$ & $\mathrm{C}(32)-\mathrm{C}(37)-\mathrm{H}(37)$ & 126.3 \\
\hline $\mathrm{C}(26)-\mathrm{C}(25)-\mathrm{I}(2)$ & $120.1(8)$ & $\mathrm{C}(45)-\mathrm{C}(38)-\mathrm{H}(38 \mathrm{~A})$ & 109.5 \\
\hline $\mathrm{C}(24)-\mathrm{C}(25)-\mathrm{I}(2)$ & $120.1(8)$ & $\mathrm{C}(45)-\mathrm{C}(38)-\mathrm{H}(38 \mathrm{~B})$ & 109.5 \\
\hline$C(25)-C(26)-C(27)$ & $120.8(10)$ & $\mathrm{H}(38 \mathrm{~A})-\mathrm{C}(38)-\mathrm{H}(38 \mathrm{~B})$ & 109.5 \\
\hline $\mathrm{C}(25)-\mathrm{C}(26)-\mathrm{H}(26)$ & 119.6 & $\mathrm{C}(45)-\mathrm{C}(38)-\mathrm{H}(38 \mathrm{C})$ & 109.5 \\
\hline $\mathrm{C}(27)-\mathrm{C}(26)-\mathrm{H}(26)$ & 119.6 & $\mathrm{H}(38 \mathrm{~A})-\mathrm{C}(38)-\mathrm{H}(38 \mathrm{C})$ & 109.5 \\
\hline$C(26)-C(27)-C(28)$ & $120.9(10)$ & $\mathrm{H}(38 \mathrm{~B})-\mathrm{C}(38)-\mathrm{H}(38 \mathrm{C})$ & 109.5 \\
\hline $\mathrm{C}(26)-\mathrm{C}(27)-\mathrm{H}(27)$ & 119.5 & $\mathrm{C}(45)-\mathrm{C}(39)-\mathrm{H}(39 \mathrm{~A})$ & 109.5 \\
\hline $\mathrm{C}(28)-\mathrm{C}(27)-\mathrm{H}(27)$ & 119.5 & $\mathrm{C}(45)-\mathrm{C}(39)-\mathrm{H}(39 \mathrm{~B})$ & 109.5 \\
\hline$C(27)-C(28)-C(29)$ & $116.7(9)$ & H(39A)-C(39)-H(39B) & 109.5 \\
\hline$C(27)-C(28)-C(35)$ & 121.1(9) & $\mathrm{C}(45)-\mathrm{C}(39)-\mathrm{H}(39 \mathrm{C})$ & 109.5 \\
\hline$C(29)-C(28)-C(35)$ & $122.2(9)$ & $\mathrm{H}(39 \mathrm{~A})-\mathrm{C}(39)-\mathrm{H}(39 \mathrm{C})$ & 109.5 \\
\hline $\mathrm{C}(24)-\mathrm{C}(29)-\mathrm{C}(28)$ & $121.8(10)$ & H(39B)-C(39)-H(39C) & 109.5 \\
\hline $\mathrm{C}(24)-\mathrm{C}(29)-\mathrm{H}(29)$ & 119.1 & $\mathrm{C}(45)-\mathrm{C}(40)-\mathrm{H}(40 \mathrm{~A})$ & 109.5 \\
\hline $\mathrm{C}(28)-\mathrm{C}(29)-\mathrm{H}(29)$ & 119.1 & $\mathrm{C}(45)-\mathrm{C}(40)-\mathrm{H}(40 \mathrm{~B})$ & 109.5 \\
\hline$C(31)-C(30)-C(35)$ & $121.4(9)$ & $\mathrm{H}(40 \mathrm{~A})-\mathrm{C}(40)-\mathrm{H}(40 \mathrm{~B})$ & 109.5 \\
\hline $\mathrm{C}(31)-\mathrm{C}(30)-\mathrm{H}(30)$ & 119.3 & $\mathrm{C}(45)-\mathrm{C}(40)-\mathrm{H}(40 \mathrm{C})$ & 109.5 \\
\hline $\mathrm{C}(35)-\mathrm{C}(30)-\mathrm{H}(30)$ & 119.3 & $\mathrm{H}(40 \mathrm{~A})-\mathrm{C}(40)-\mathrm{H}(40 \mathrm{C})$ & 109.5 \\
\hline$C(30)-C(31)-C(32)$ & 119.2(9) & $\mathrm{H}(40 \mathrm{~B})-\mathrm{C}(40)-\mathrm{H}(40 \mathrm{C})$ & 109.5 \\
\hline $\mathrm{C}(30)-\mathrm{C}(31)-\mathrm{H}(31)$ & 120.4 & $C(44)-C(41)-C(42)$ & $109.9(10)$ \\
\hline $\mathrm{C}(32)-\mathrm{C}(31)-\mathrm{H}(31)$ & 120.4 & $C(44)-C(41)-C(43)$ & 108.1(9) \\
\hline$C(31)-C(32)-C(33)$ & $119.8(9)$ & $C(42)-C(41)-C(43)$ & $108.6(10)$ \\
\hline
\end{tabular}




$\begin{array}{llll}\mathrm{C}(44)-\mathrm{C}(41)-\mathrm{P}(2) & 117.3(8) & \mathrm{C}(39)-\mathrm{C}(45)-\mathrm{C}(40) & 110.7(9) \\ \mathrm{C}(42)-\mathrm{C}(41)-\mathrm{P}(2) & 107.5(7) & \mathrm{C}(38)-\mathrm{C}(45)-\mathrm{P}(2) & 104.6(7) \\ \mathrm{C}(43)-\mathrm{C}(41)-\mathrm{P}(2) & 105.0(7) & \mathrm{C}(39)-\mathrm{C}(45)-\mathrm{P}(2) & 114.9(7) \\ \mathrm{C}(41)-\mathrm{C}(42)-\mathrm{H}(42 \mathrm{~A}) & 109.5 & \mathrm{C}(40)-\mathrm{C}(45)-\mathrm{P}(2) & 109.2(7) \\ \mathrm{C}(41)-\mathrm{C}(42)-\mathrm{H}(42 \mathrm{~B}) & 109.5 & \mathrm{C}(14)-\mathrm{N}(1)-\mathrm{C}(11) & 106.5(8) \\ \mathrm{H}(42 \mathrm{~A})-\mathrm{C}(42)-\mathrm{H}(42 \mathrm{~B}) & 109.5 & \mathrm{C}(14)-\mathrm{N}(1)-\mathrm{P}(1) & 126.2(7) \\ \mathrm{C}(41)-\mathrm{C}(42)-\mathrm{H}(42 \mathrm{C}) & 109.5 & \mathrm{C}(11)-\mathrm{N}(1)-\mathrm{P}(1) & 126.4(7) \\ \mathrm{H}(42 \mathrm{~A})-\mathrm{C}(42)-\mathrm{H}(42 \mathrm{C}) & 109.5 & \mathrm{C}(36)-\mathrm{N}(2)-\mathrm{C}(33) & 107.0(8) \\ \mathrm{H}(42 \mathrm{~B})-\mathrm{C}(42)-\mathrm{H}(42 \mathrm{C}) & 109.5 & \mathrm{C}(36)-\mathrm{N}(2)-\mathrm{P}(2) & 126.9(7) \\ \mathrm{C}(41)-\mathrm{C}(43)-\mathrm{H}(43 \mathrm{~A}) & 109.5 & \mathrm{C}(33)-\mathrm{N}(2)-\mathrm{P}(2) & 125.5(7) \\ \mathrm{C}(41)-\mathrm{C}(43)-\mathrm{H}(43 \mathrm{~B}) & 109.5 & \mathrm{O}(1)-\mathrm{P}(1)-\mathrm{N}(1) & 110.2(4) \\ \mathrm{H}(43 \mathrm{~A})-\mathrm{C}(43)-\mathrm{H}(43 \mathrm{~B}) & 109.5 & \mathrm{O}(1)-\mathrm{P}(1)-\mathrm{C}(15) & 110.1(5) \\ \mathrm{C}(41)-\mathrm{C}(43)-\mathrm{H}(43 \mathrm{C}) & 109.5 & \mathrm{~N}(1)-\mathrm{P}(1)-\mathrm{C}(15) & 105.7(5) \\ \mathrm{H}(43 \mathrm{~A})-\mathrm{C}(43)-\mathrm{H}(43 \mathrm{C}) & 109.5 & \mathrm{O}(1)-\mathrm{P}(1)-\mathrm{C}(19) & 109.5(5) \\ \mathrm{H}(43 \mathrm{~B})-\mathrm{C}(43)-\mathrm{H}(43 \mathrm{C}) & 109.5 & \mathrm{~N}(1)-\mathrm{P}(1)-\mathrm{C}(19) & 105.1(5) \\ \mathrm{C}(41)-\mathrm{C}(44)-\mathrm{H}(44 \mathrm{~A}) & 109.5 & \mathrm{C}(15)-\mathrm{P}(1)-\mathrm{C}(19) & 116.0(5) \\ \mathrm{C}(41)-\mathrm{C}(44)-\mathrm{H}(44 \mathrm{~B}) & 109.5 & \mathrm{O}(2)-\mathrm{P}(2)-\mathrm{N}(2) & 109.6(4) \\ \mathrm{H}(44 \mathrm{~A})-\mathrm{C}(44)-\mathrm{H}(44 \mathrm{~B}) & 109.5 & \mathrm{O}(2)-\mathrm{P}(2)-\mathrm{C}(45) & 110.2(4) \\ \mathrm{C}(41)-\mathrm{C}(44)-\mathrm{H}(44 \mathrm{C}) & 109.5 & \mathrm{~N}(2)-\mathrm{P}(2)-\mathrm{C}(45) & 104.6(4) \\ \mathrm{H}(44 \mathrm{~A})-\mathrm{C}(44)-\mathrm{H}(44 \mathrm{C}) & 109.5 & \mathrm{O}(2)-\mathrm{P}(2)-\mathrm{C}(41) & 110.0(5) \\ \mathrm{H}(44 \mathrm{~B})-\mathrm{C}(44)-\mathrm{H}(44 \mathrm{C}) & 109.5 & \mathrm{~N}(2)-\mathrm{P}(2)-\mathrm{C}(41) & 107.2(4) \\ \mathrm{C}(38)-\mathrm{C}(45)-\mathrm{C}(39) & 108.5(9) & \mathrm{C}(45)-\mathrm{P}(2)-\mathrm{C}(41) & 114.9(5) \\ \mathrm{C}(38)-\mathrm{C}(45)-\mathrm{C}(40) & 108.7(9) & & \end{array}$




\section{References}

1. (a) Bigot, A.; Williamson, A. E.; Gaunt, M. J. J. Am. Chem. Soc. 2011, 133, 13778. (b) Ichiishi, N.; Canty, A. J.; Yates, B. F.; Sanford, M. S. Org. Lett. 2013, 15. 5134. (c) Gao, P.; Guo, W.; Xie, J. -J.; Zhao, Y.; Yuan, Y.; Xia, Y. -Z.; Shi, Z. -Z.. J. Am. Chem. Soc. 2015, 137, 12231.

2. (a) Yang, Y. -Q.; Qiu, X. -D.; Zhao, Y.; Mu, Y. -C.; Shi, Z. -Z.. J. Am. Chem. Soc. 2016, 138, 495. (b) Hartung, C. G.; Fecher, A.; Chapell, B.; Snieckus, V. Org. Lett. 2003, 5, 1899.

3. Deprez, N. R.; Kalyani, D.; Krause, A.; Sanford, M. S. J. Am. Chem. Soc. 2006, 128, 4972.

4. Phipps, R. J.; Grimster, N. P.; Gaunt, M. J. J. Am. Chem. Soc. 2008, 130, 8172. 


\section{Copies of ${ }^{1} \mathrm{H},{ }^{13} \mathrm{C},{ }^{31} \mathrm{P}$ and ${ }^{19} \mathrm{~F}$ NMR Spectra}

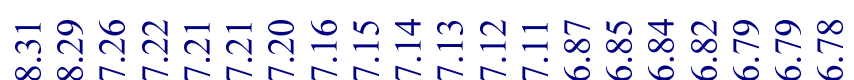

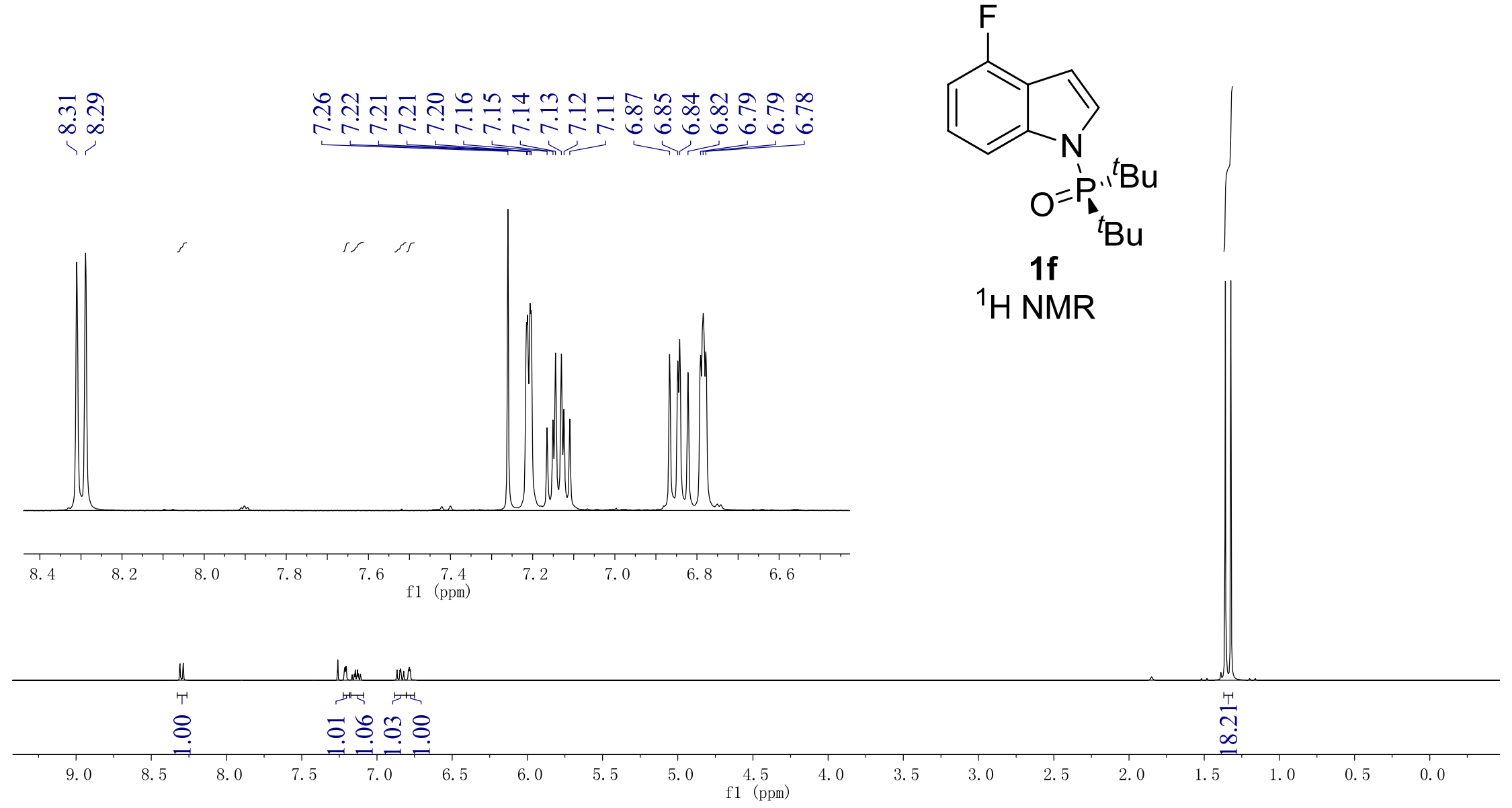

H NMR 


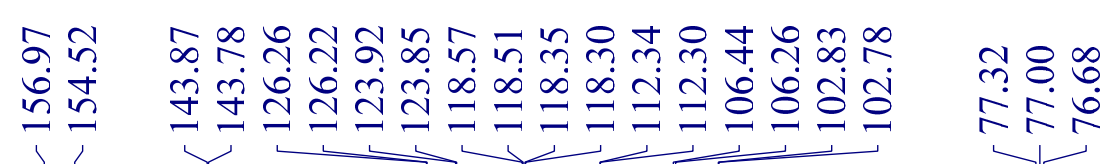
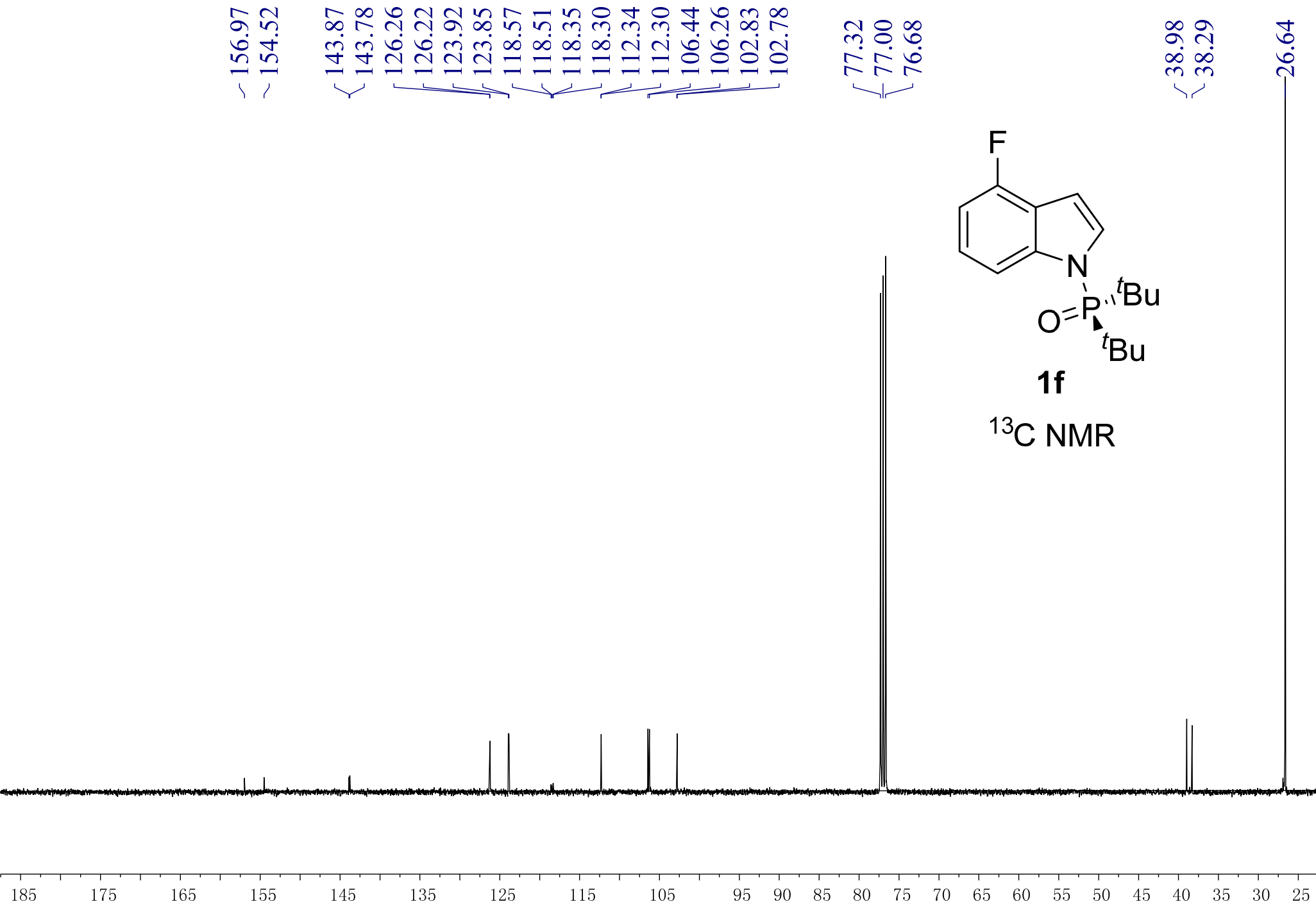

35

125

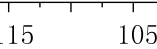

$95 \quad 90 \quad 85$ 
ธุด

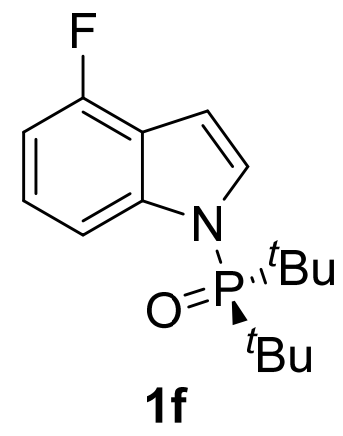

${ }^{31} \mathrm{P}$ NMR

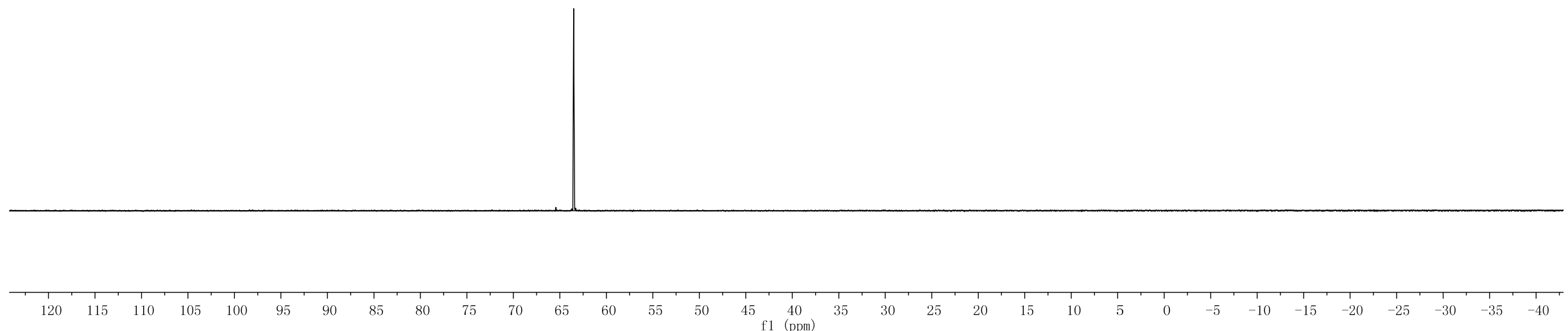



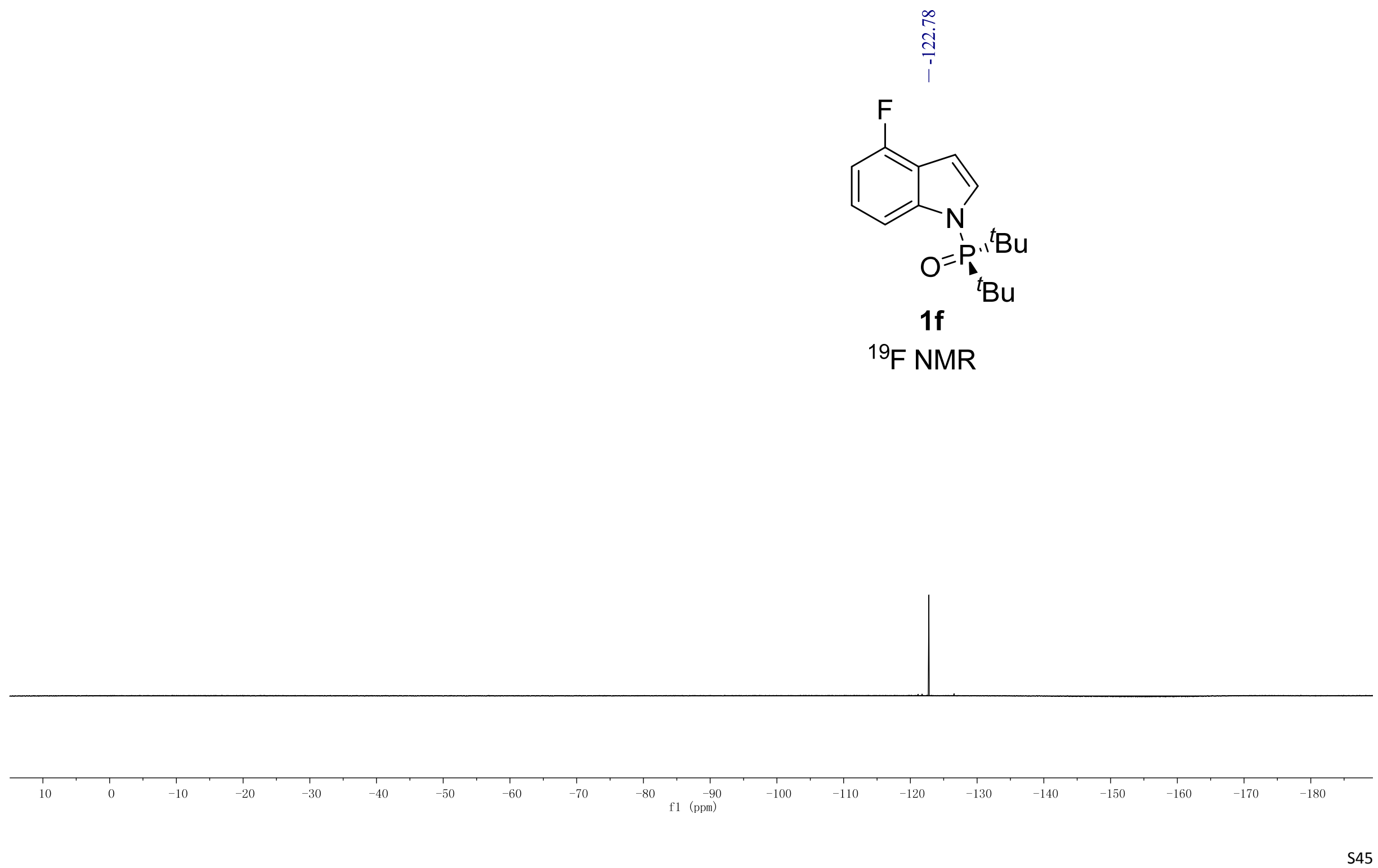


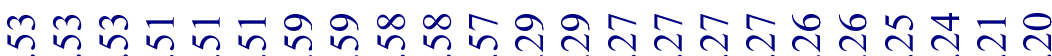

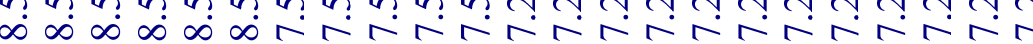
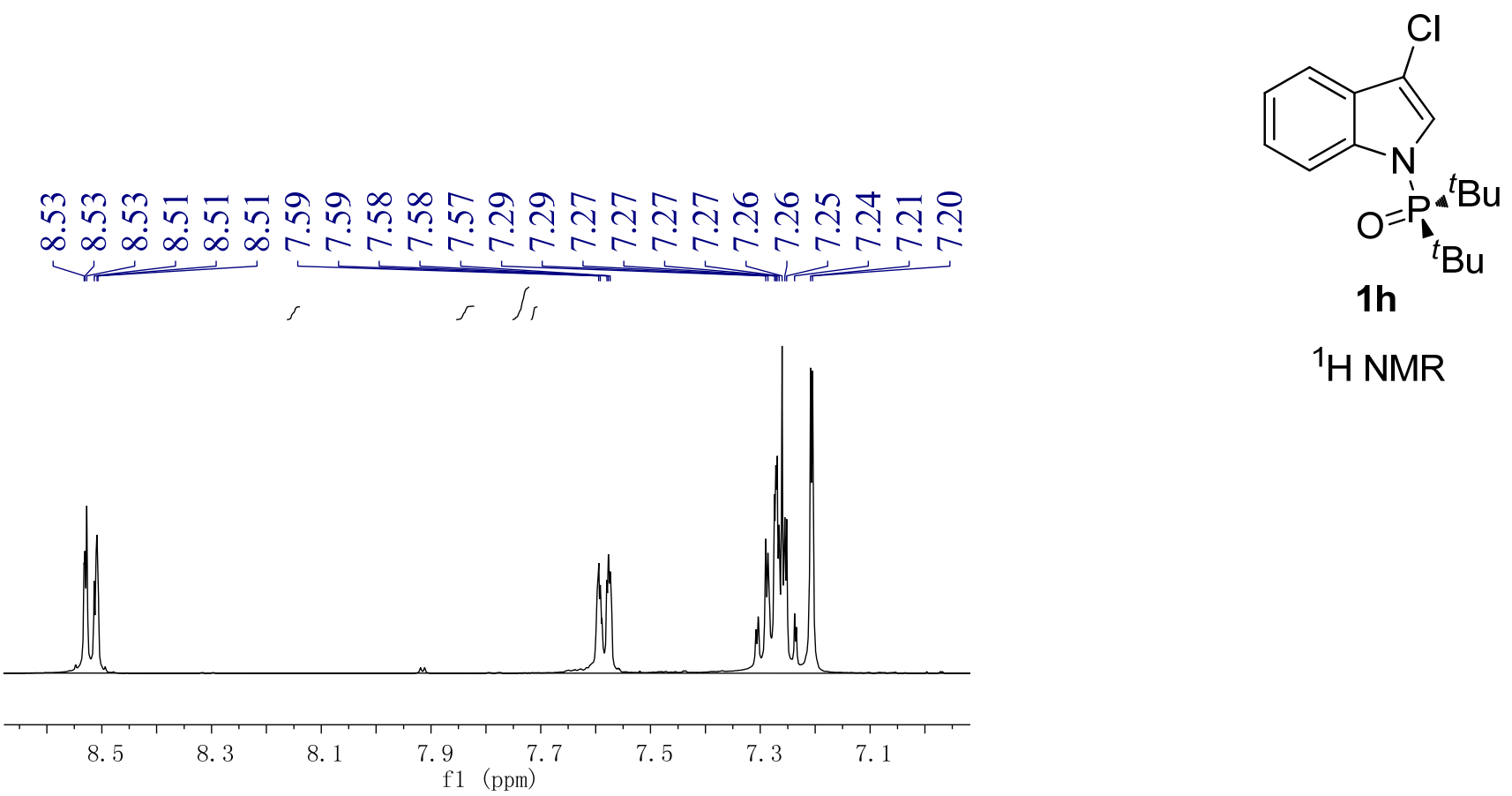

1h

${ }^{1} \mathrm{H}$ NMR

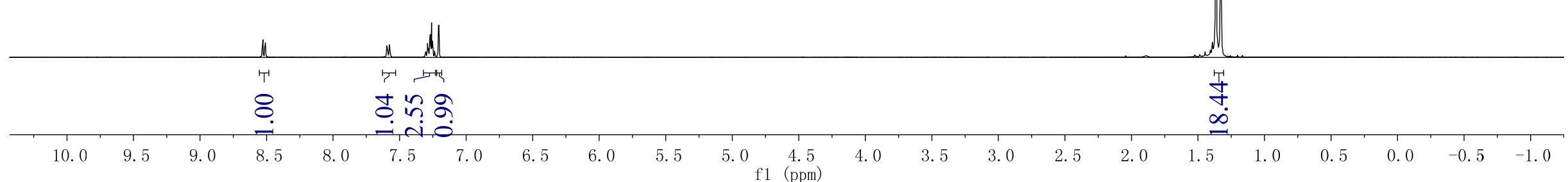




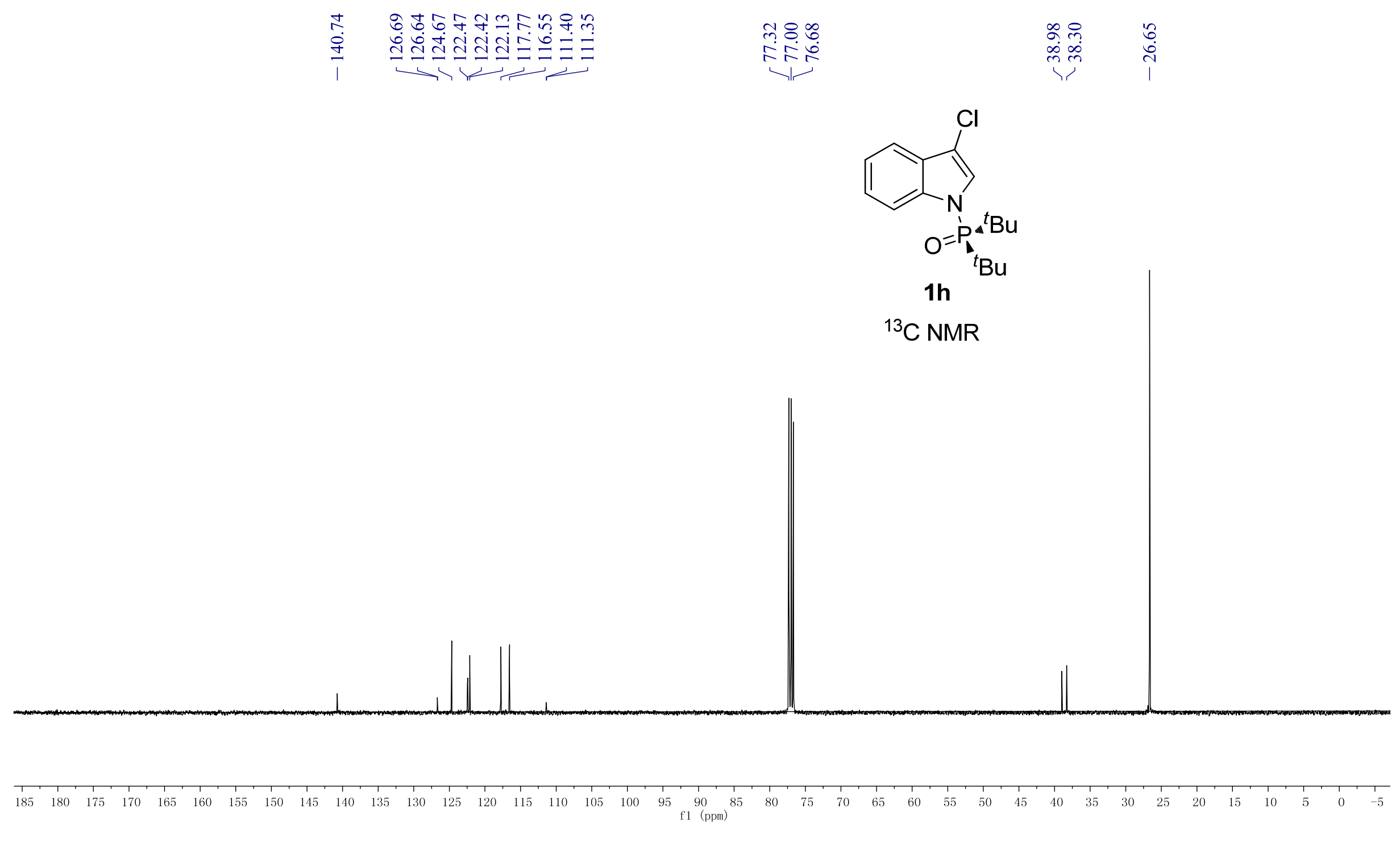


$\stackrel{\infty}{\dot{+}}$

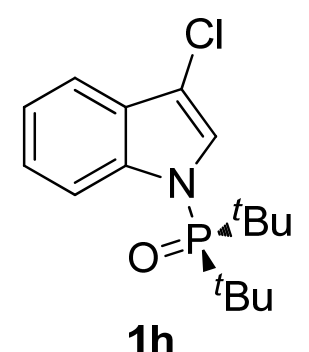

${ }^{31} \mathrm{P}$ NMR

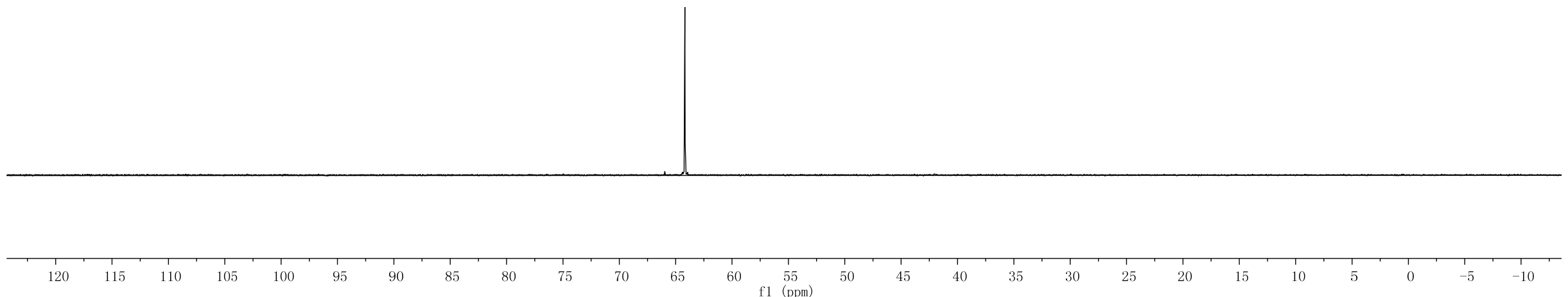



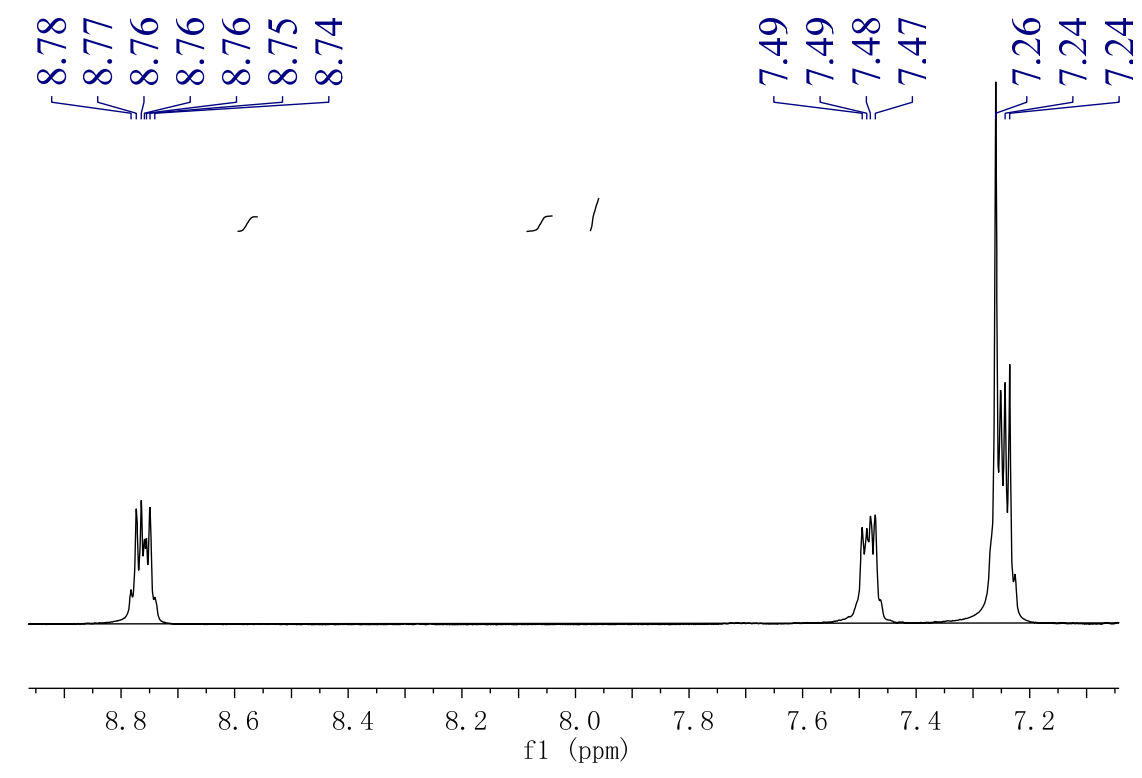

$1 \mathrm{i}$

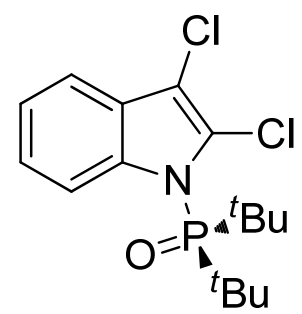

${ }^{1} \mathrm{H}$ NMR

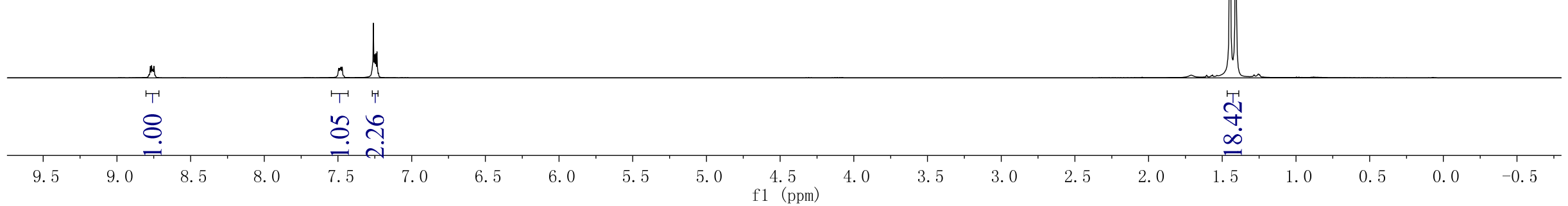




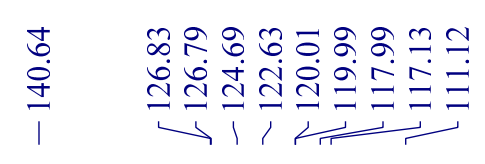

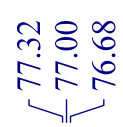

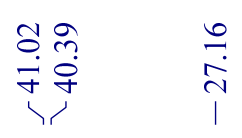

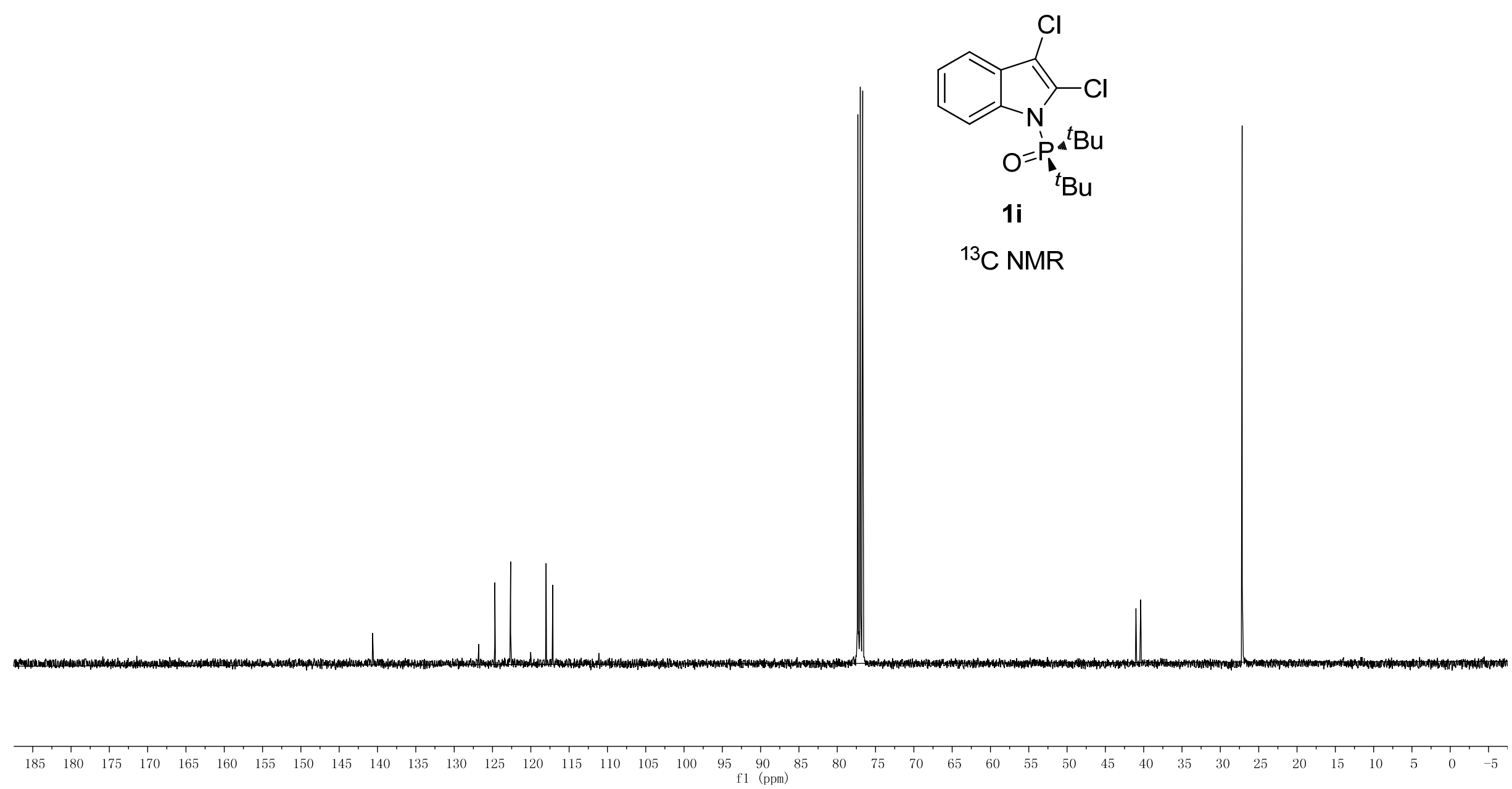




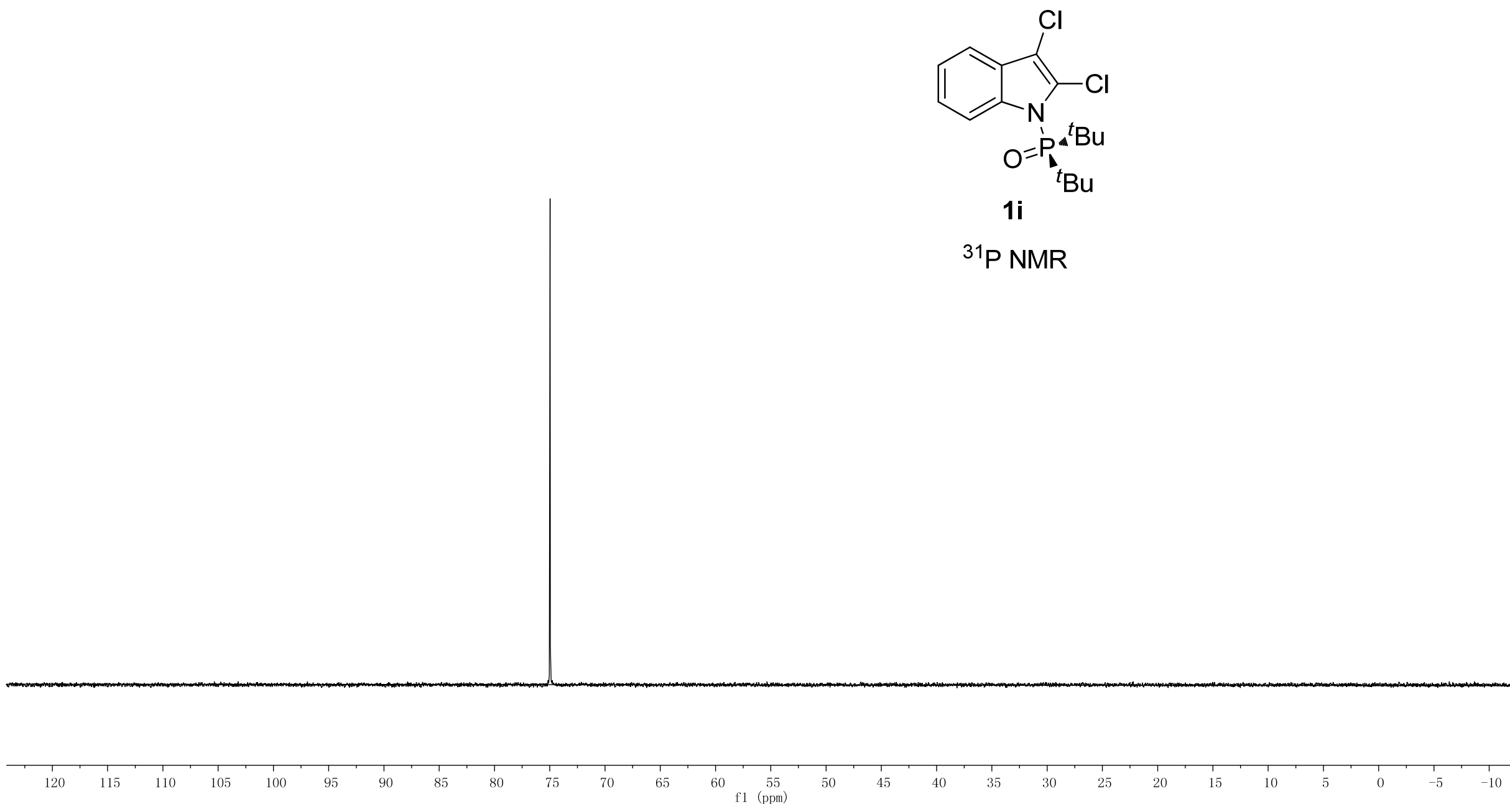




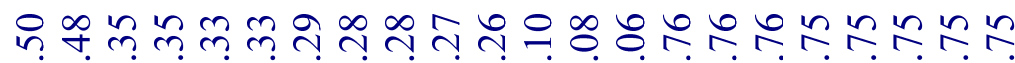

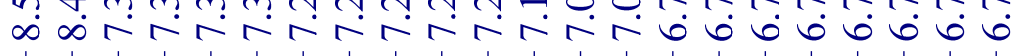

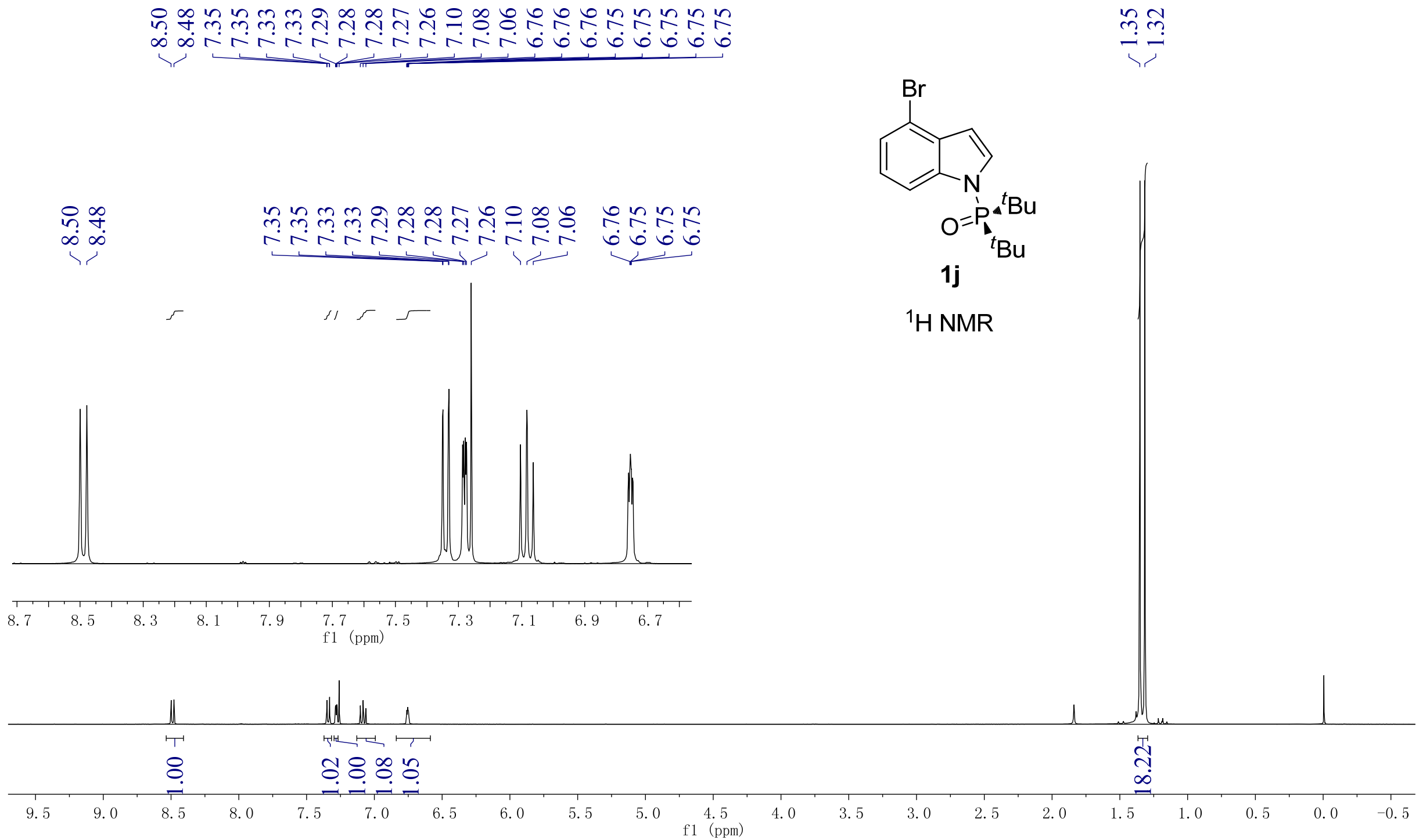




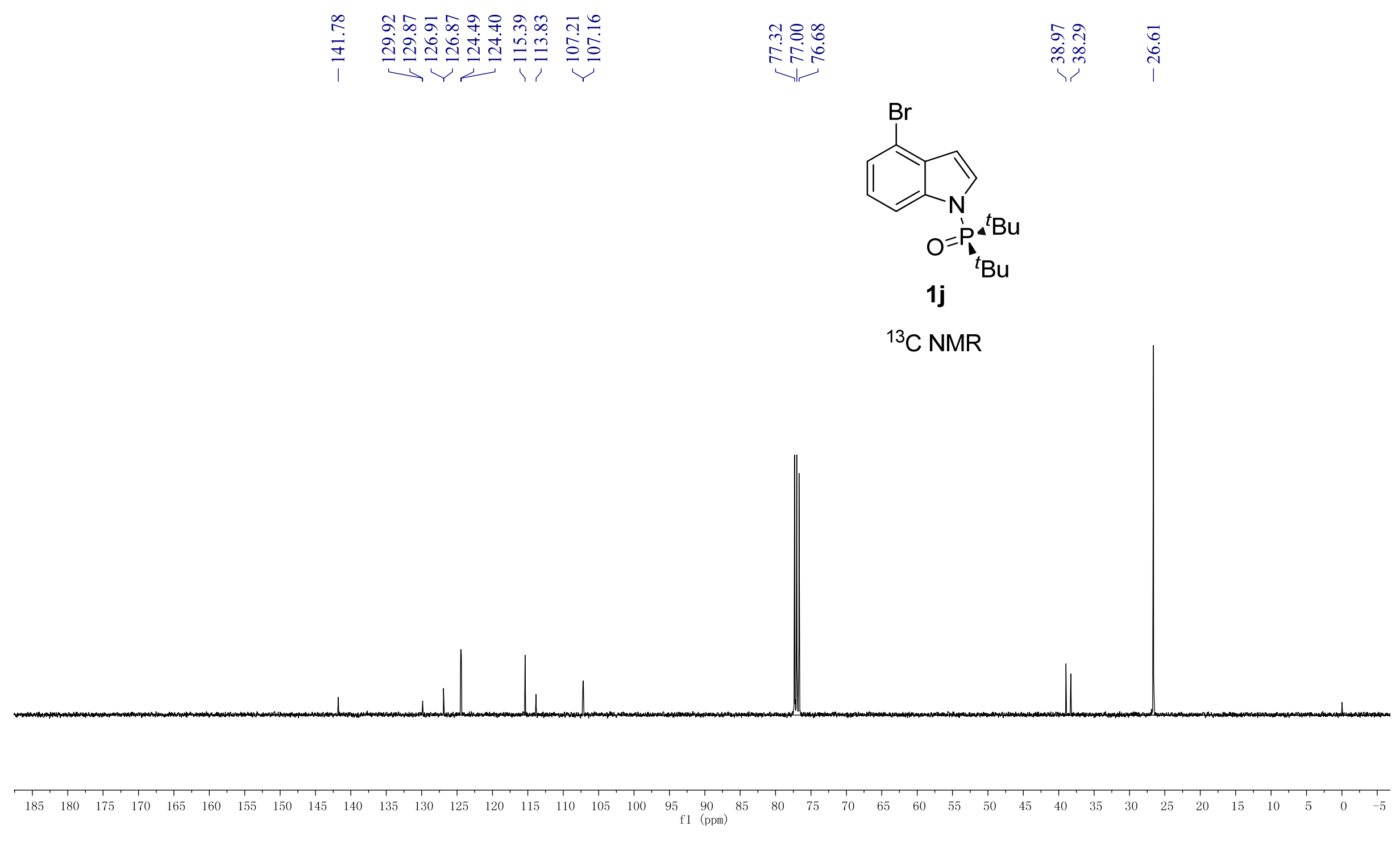


0
0
0

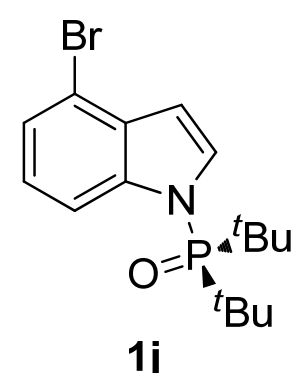

${ }^{31} \mathrm{P}$ NMR

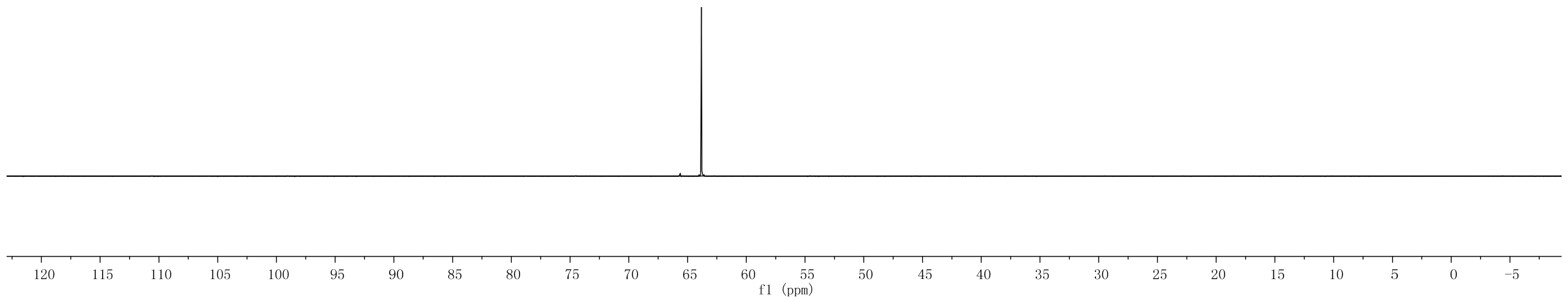



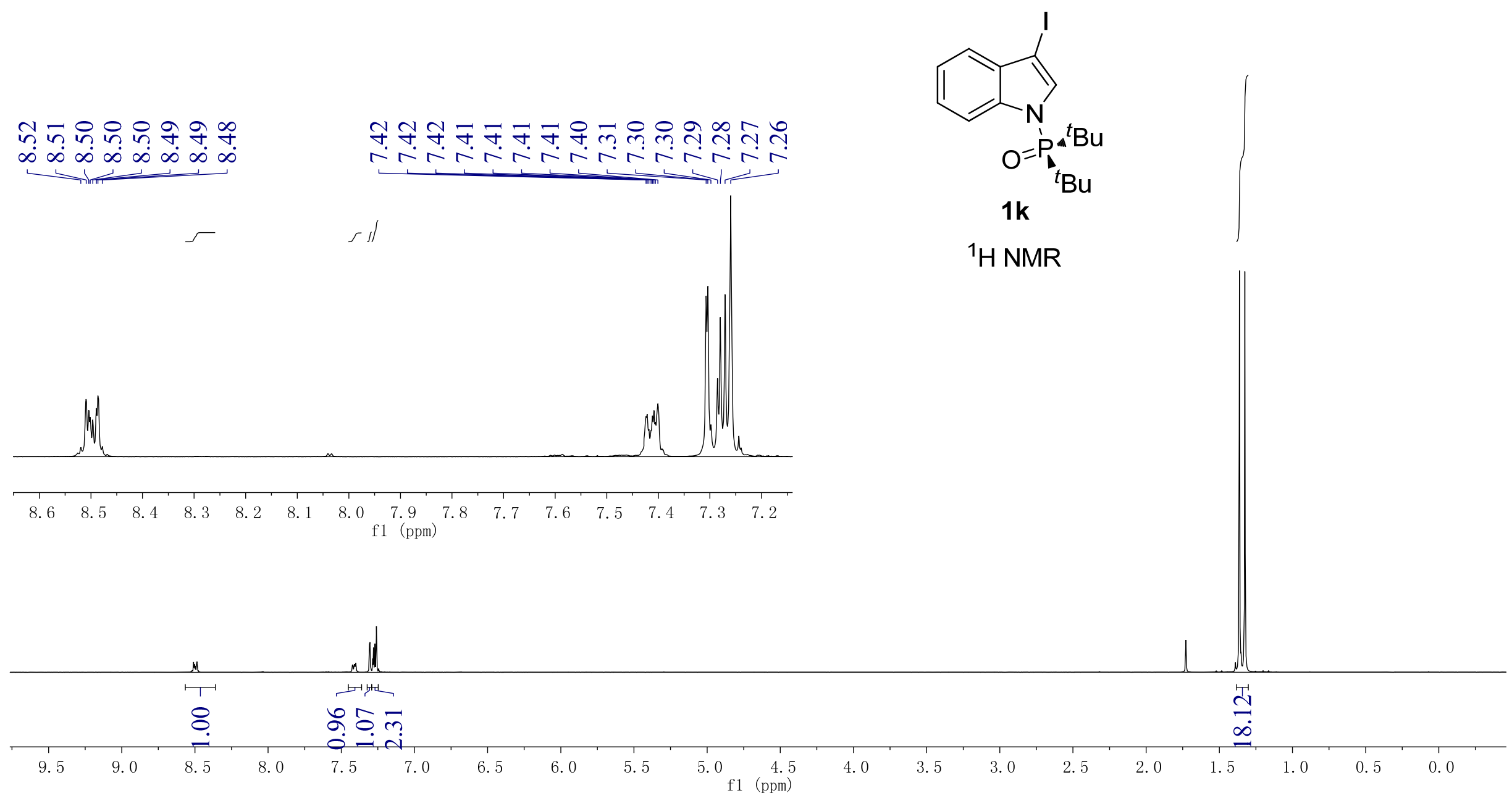

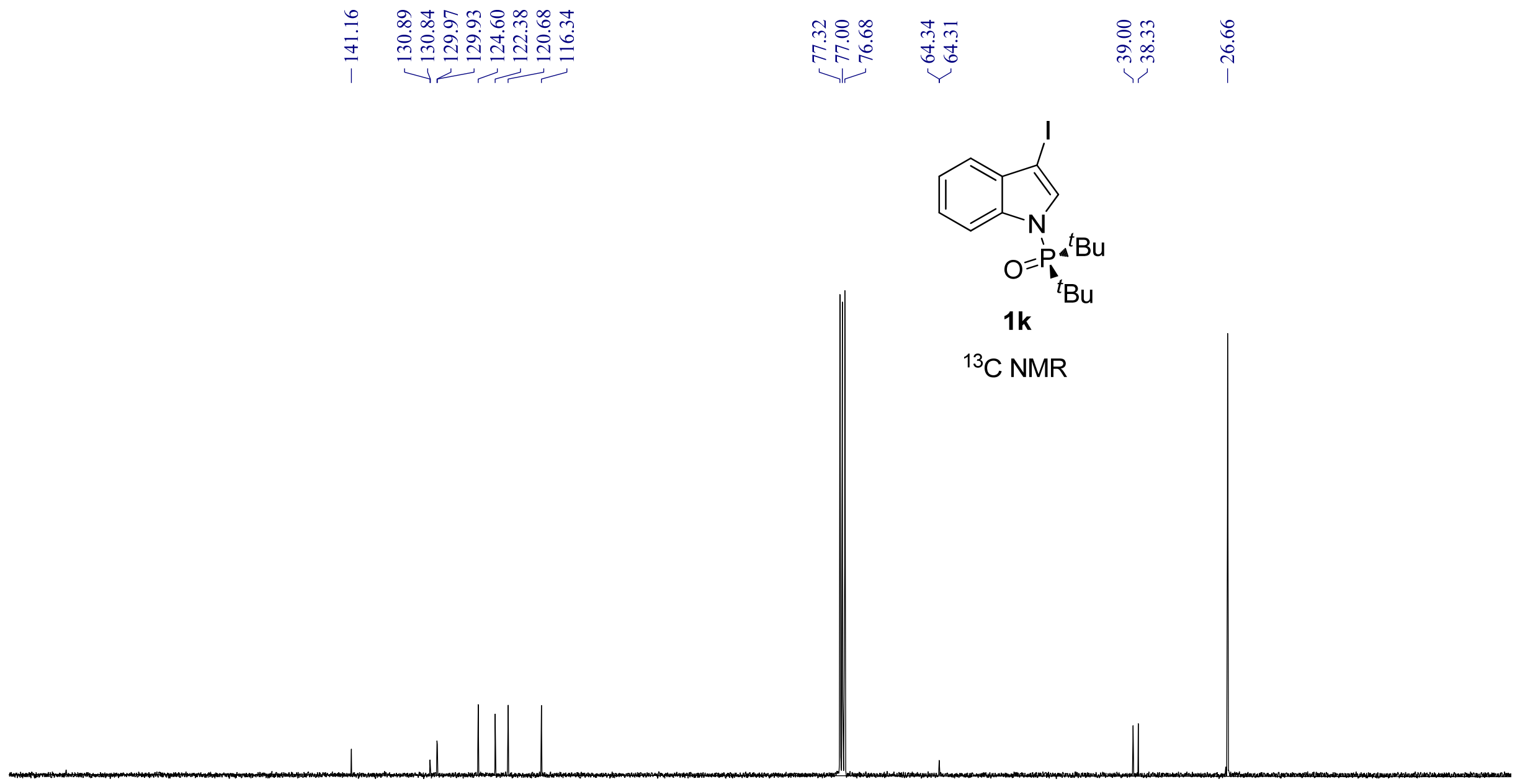

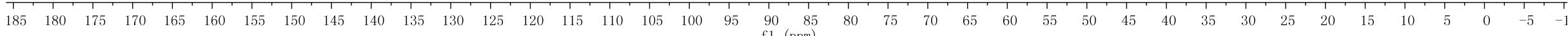




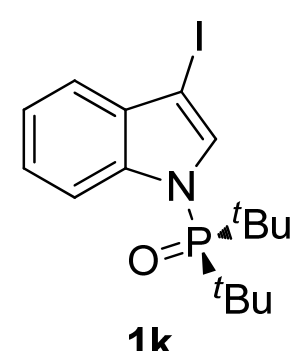

${ }^{31} \mathrm{P}$ NMR

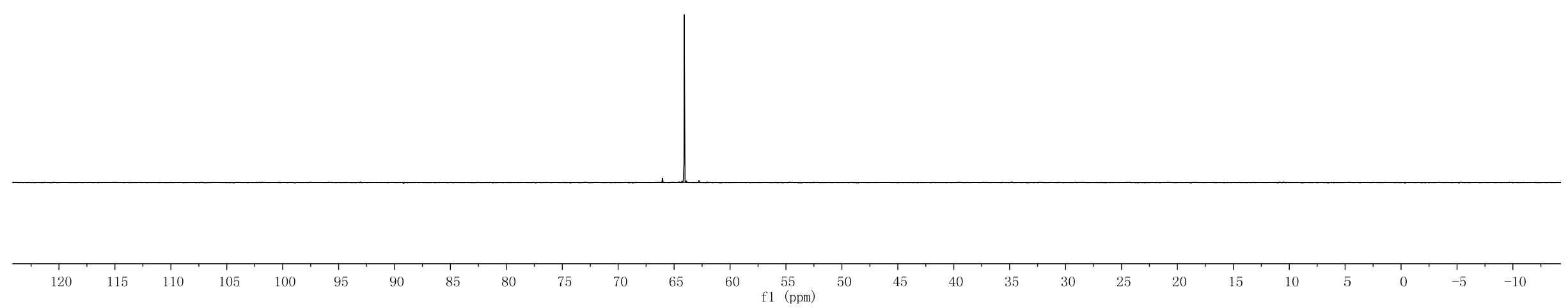




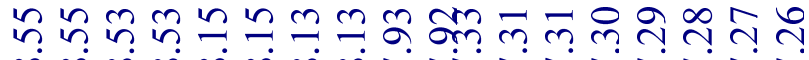

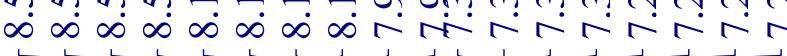

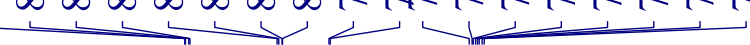

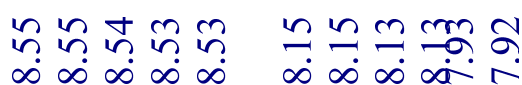

$\infty \infty \infty \infty \infty \infty) \infty \infty \infty \infty)$

, r 2
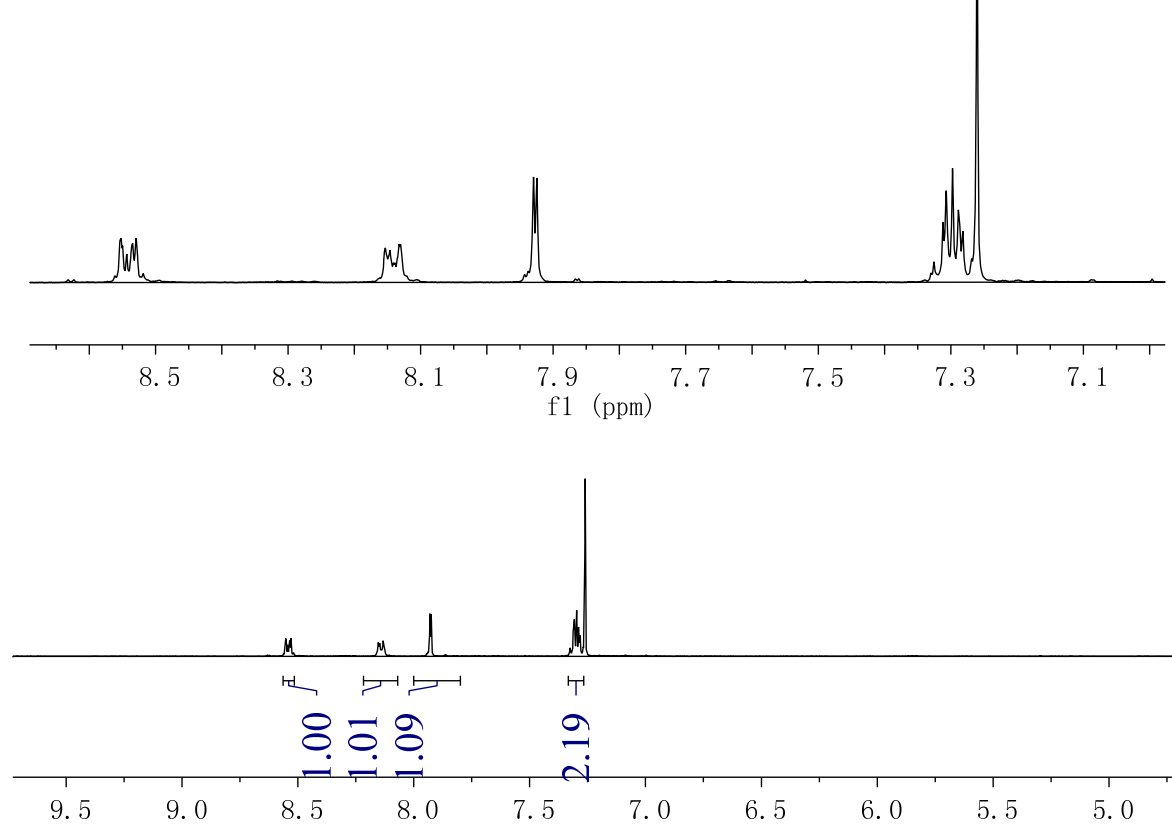

$\stackrel{a}{a}$

ตेต

i

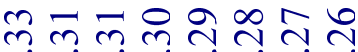

nhan han

(1)

5

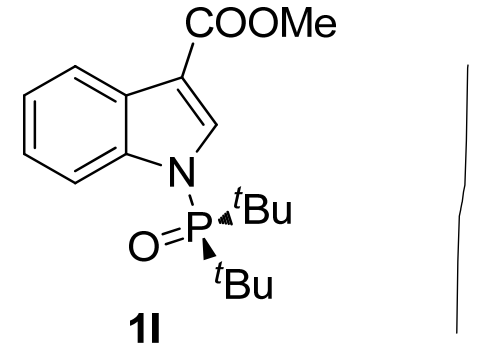

${ }^{1} \mathrm{H} N M R$

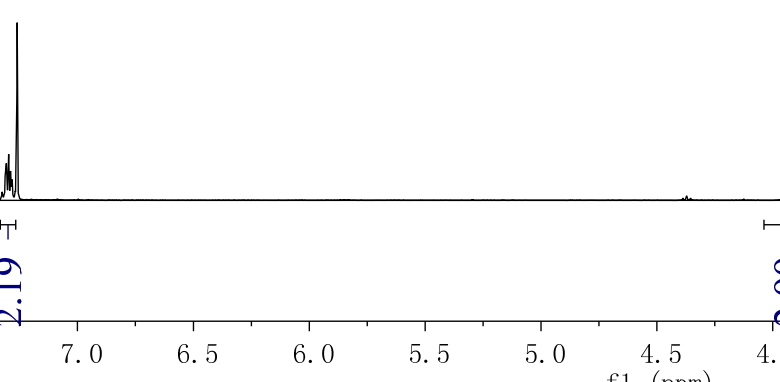



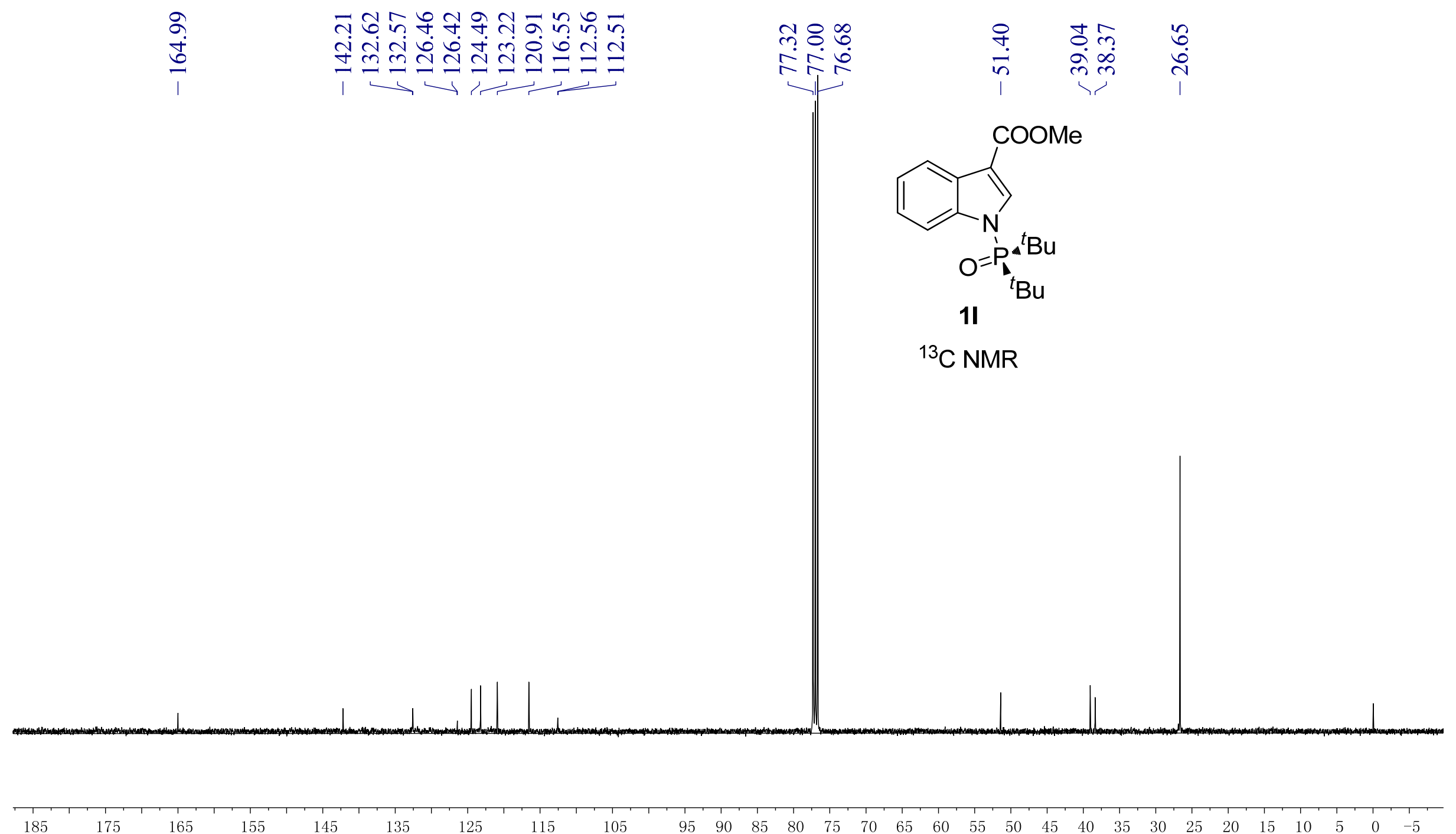
f1 (ppm) 


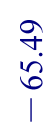

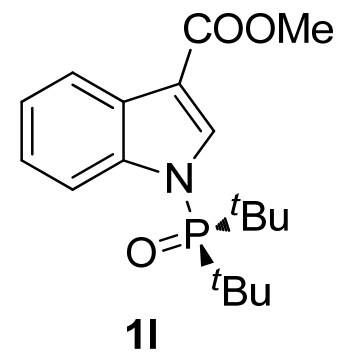

${ }^{31} \mathrm{P}$ NMR

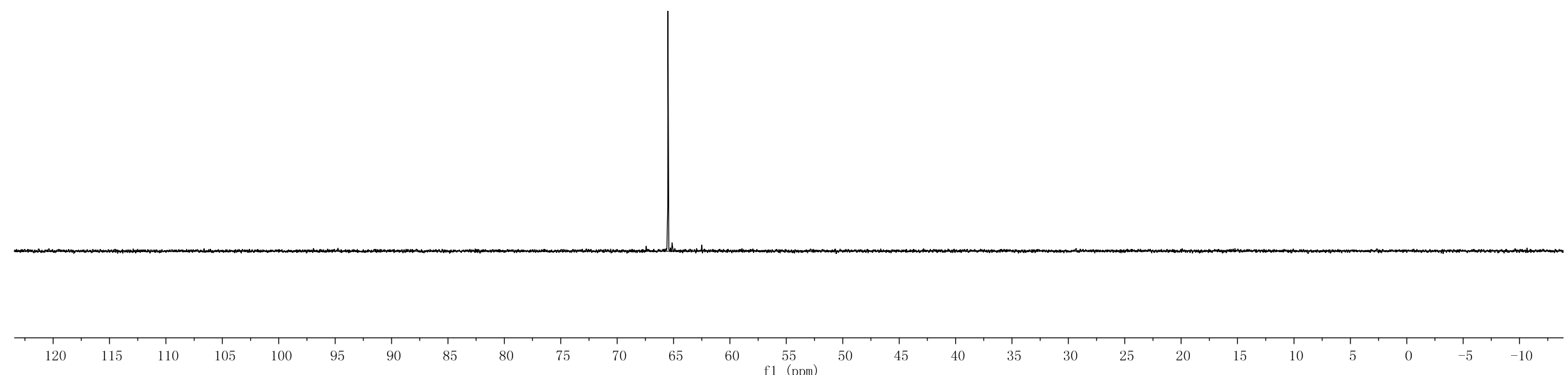




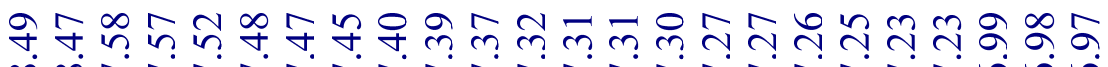

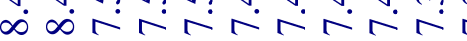
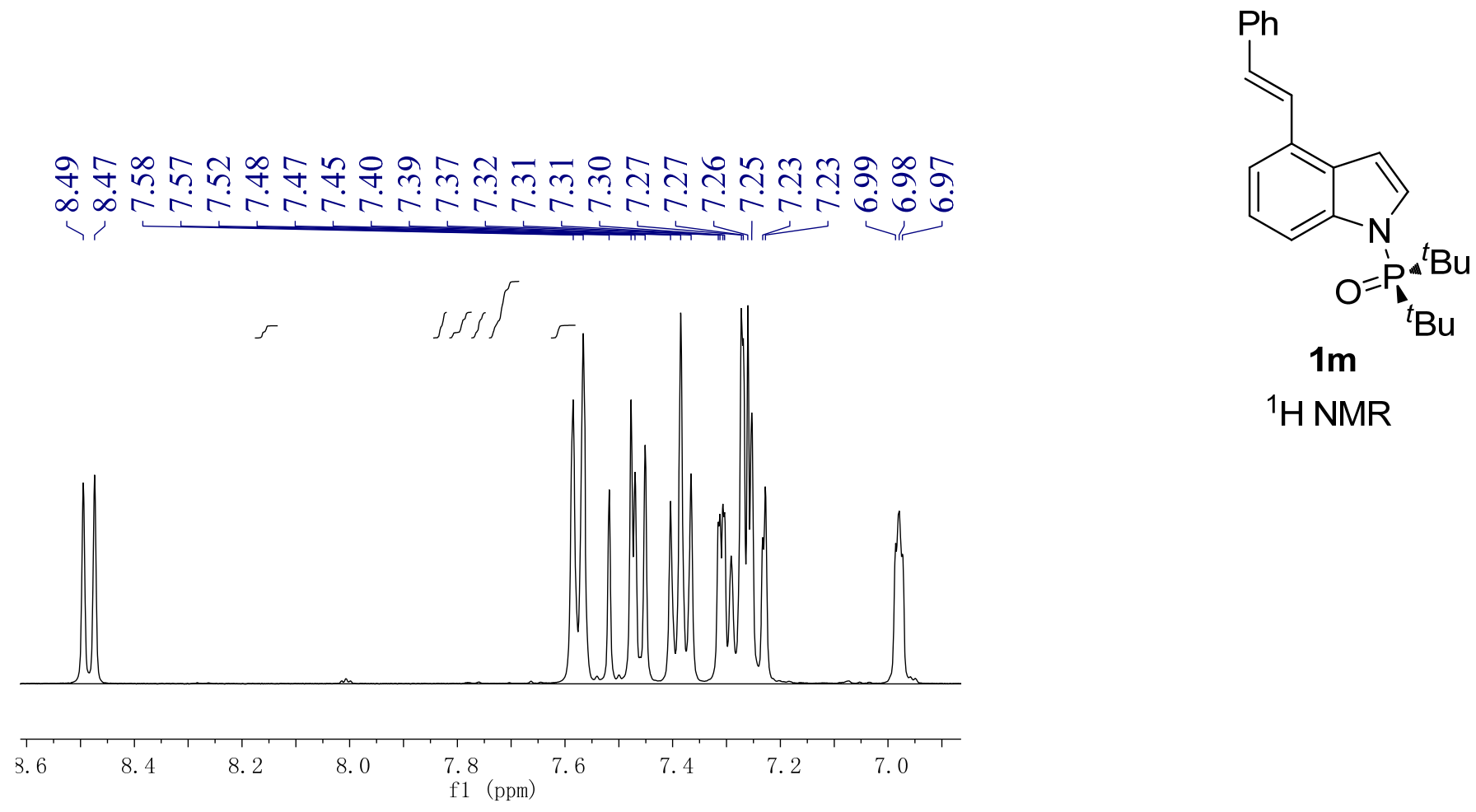

$$
1 \mathrm{~m}
$$

${ }^{1} \mathrm{H}$ NMR

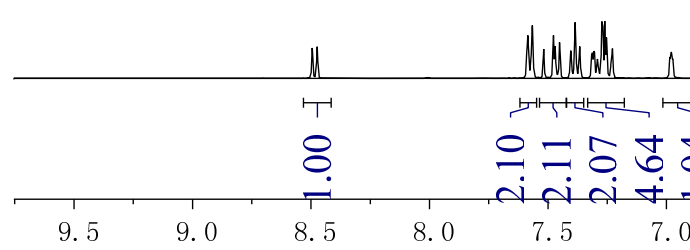

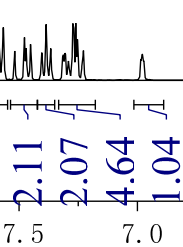

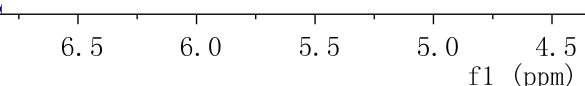

f1 ( 


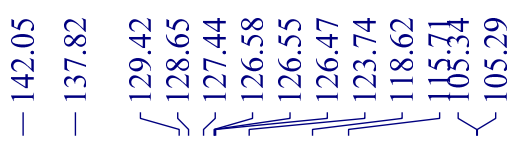

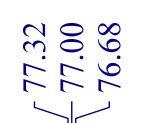

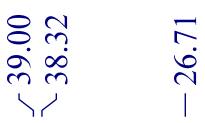

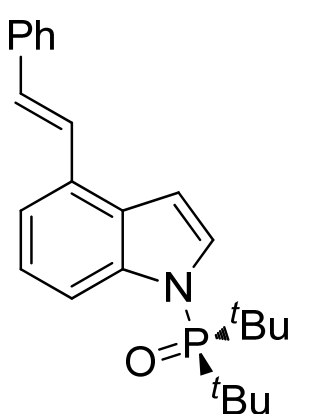

$1 \mathrm{~m}$

${ }^{13} \mathrm{C}$ NMR

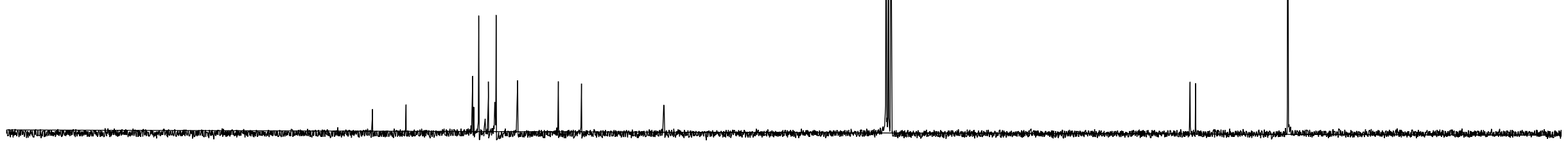

$\begin{array}{lllllllllllllllllllllllllllllllllllllllllllllllllllllllll}185 & 180 & 175 & 170 & 165 & 160 & 155 & 150 & 145 & 140 & 135 & 130 & 125 & 120 & 115 & 110 & 105 & 100 & 95 & 90 & 85 & 80 & 75 & 70 & 65 & 60 & 55 & 50 & 45 & 40 & 35 & 30 & 25 & 20 & 15 & 10 & 5 & 0 & -5\end{array}$ 
8
6
1
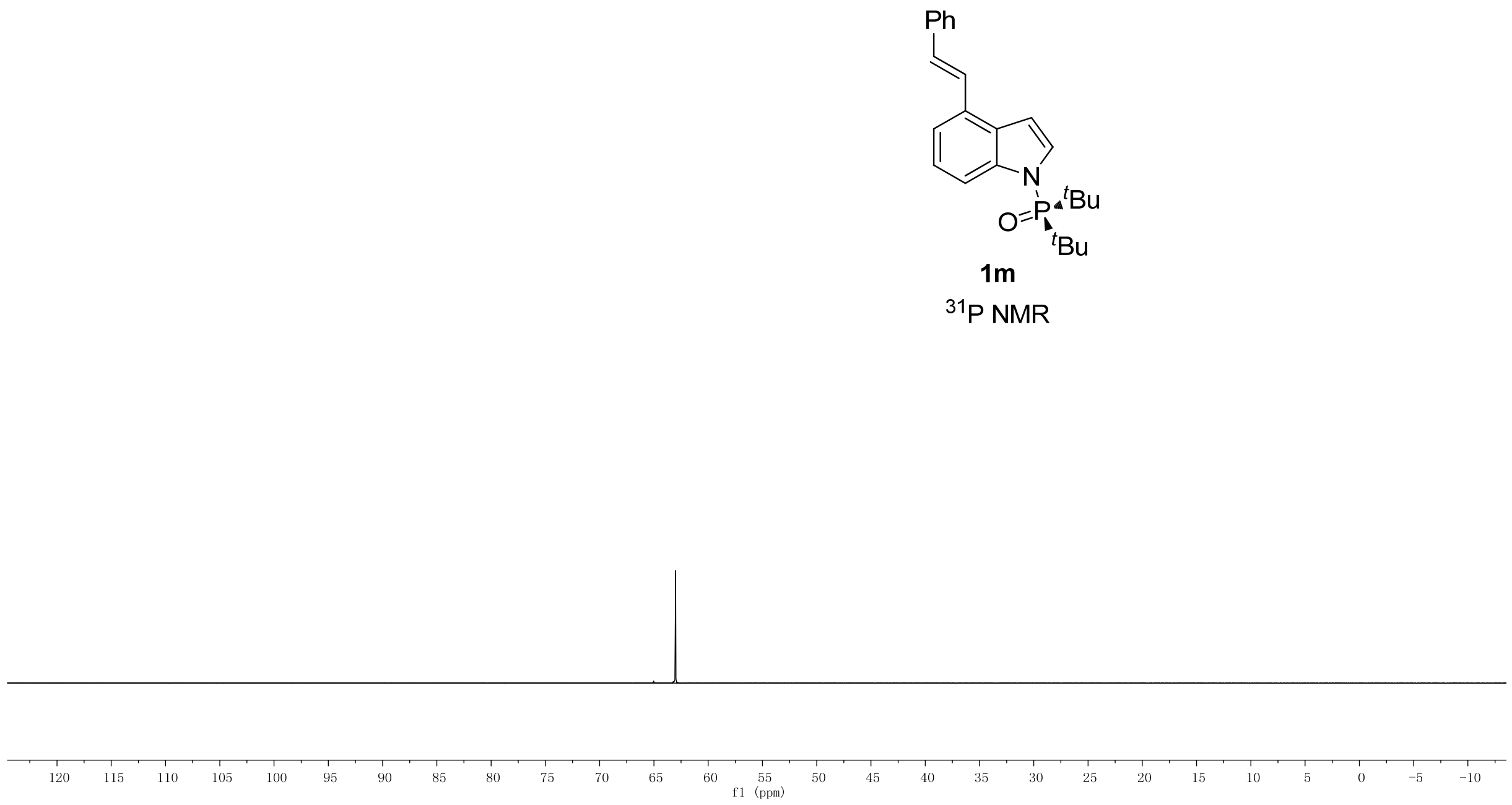

S63 
ำ่

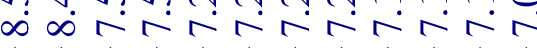
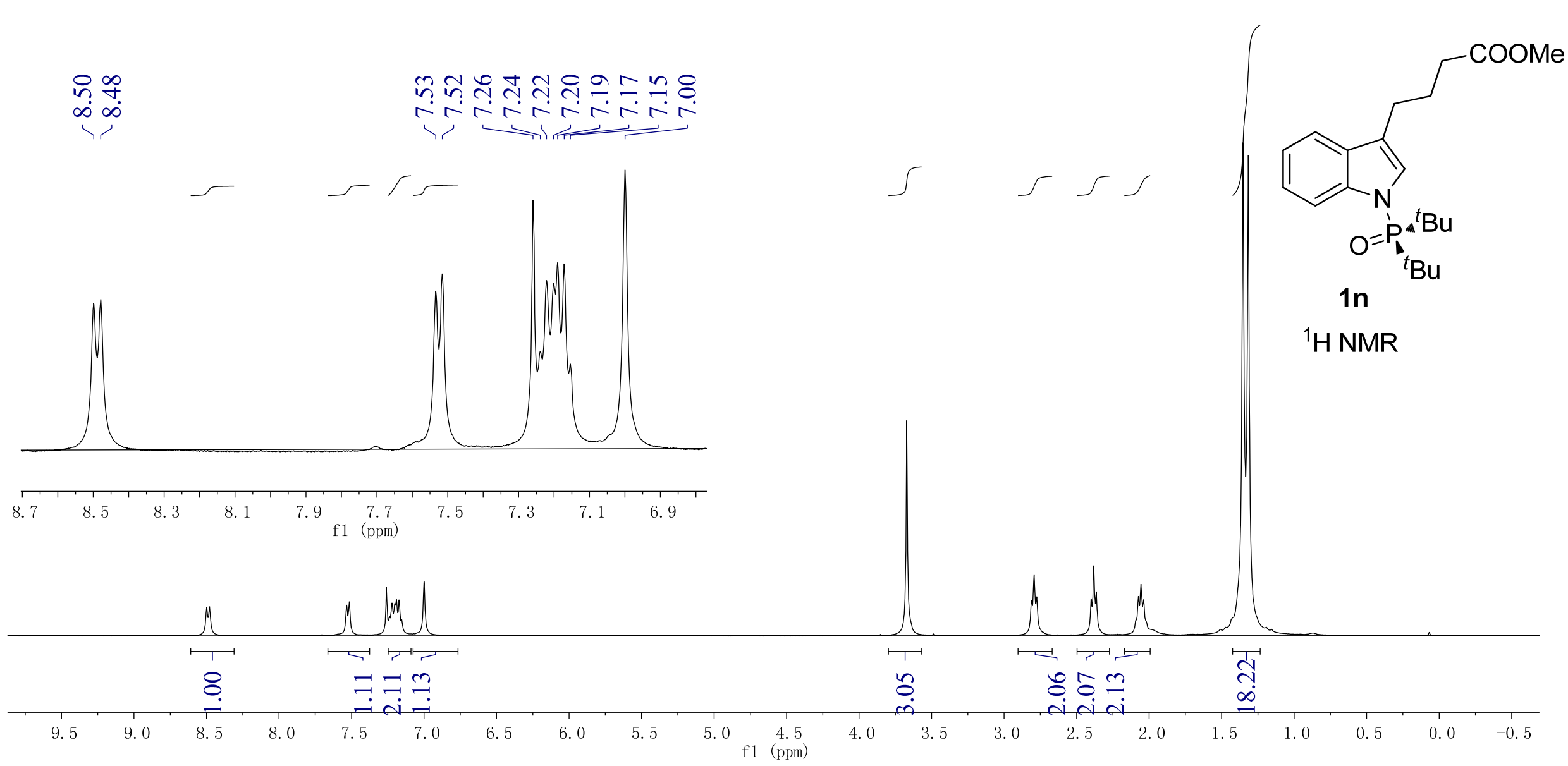

б.

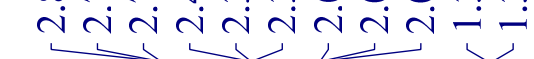



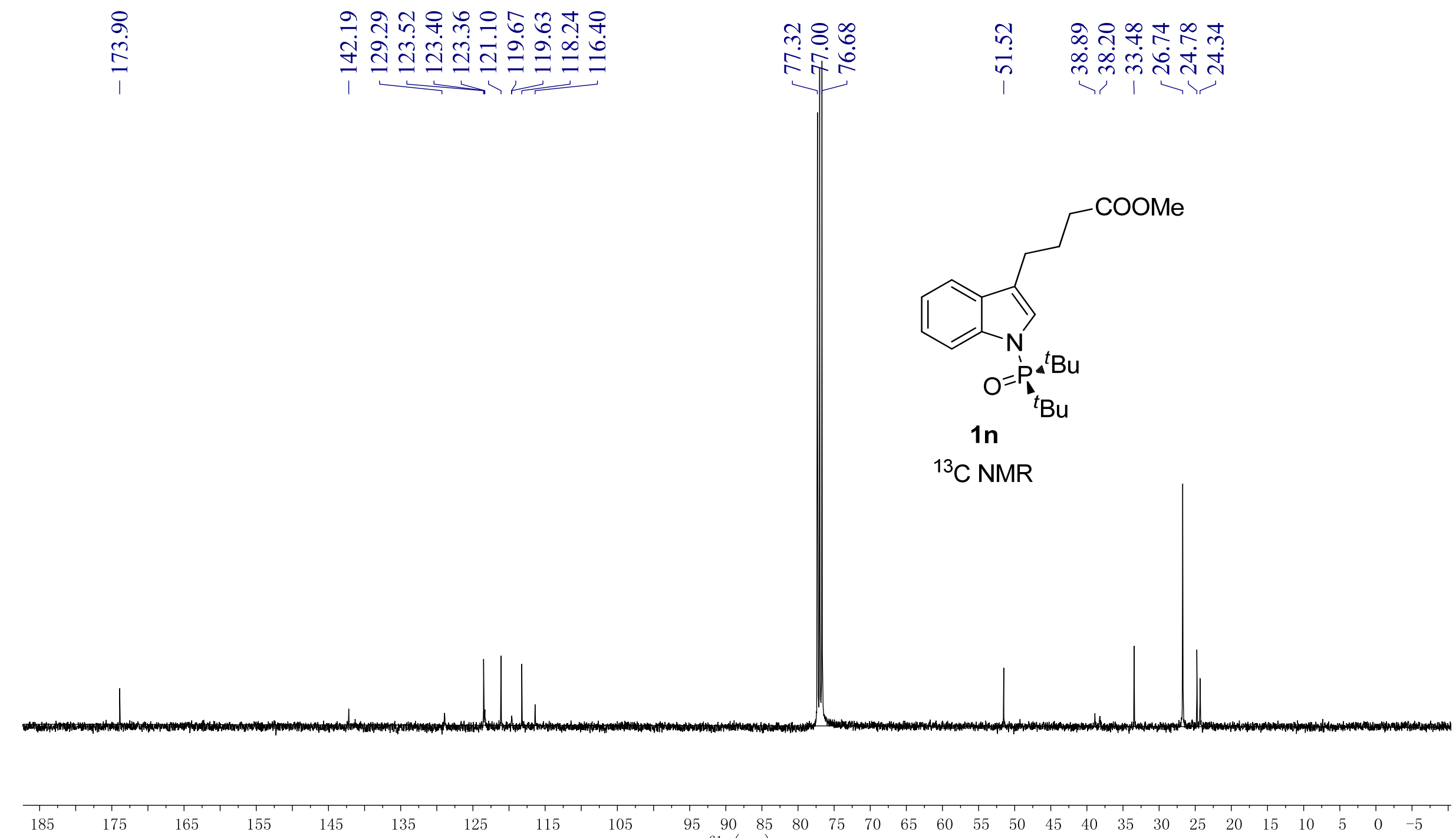


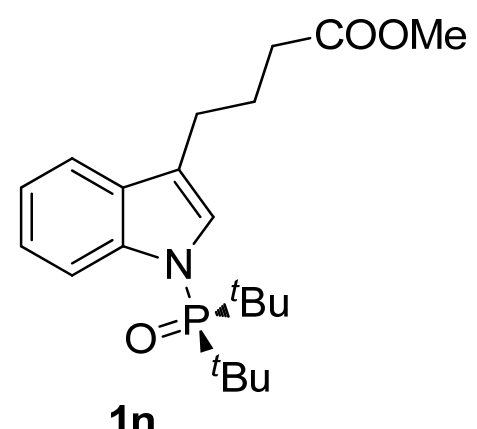

${ }^{31} \mathrm{P}$ NMR

mom 


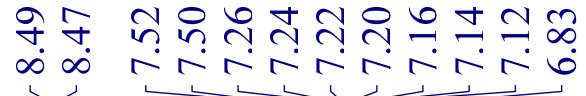

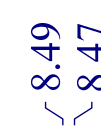

กำ กำำ

i)
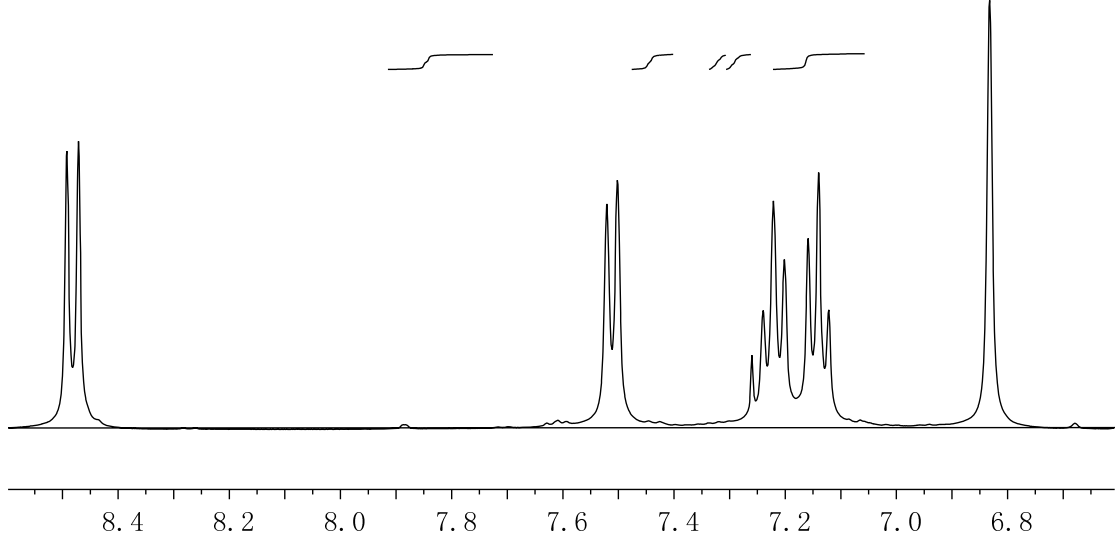

$\stackrel{7.6}{(\mathrm{ppm})}$

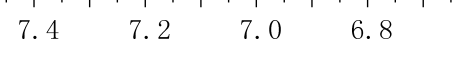

$\stackrel{\text { ঙ̊ }}{+}$

กฺุิ

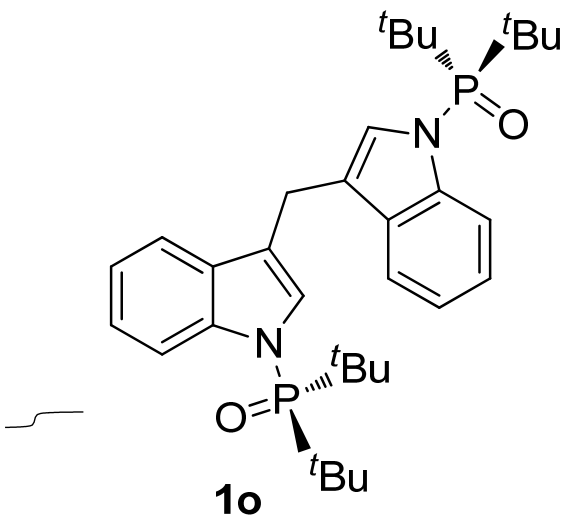

${ }^{1} \mathrm{H}$ NMR

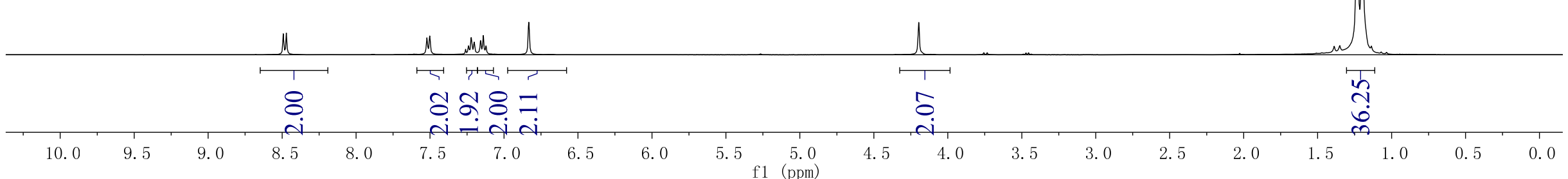




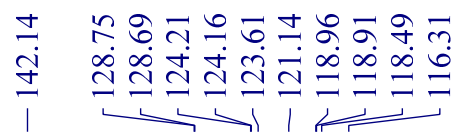

뇨용

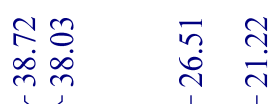

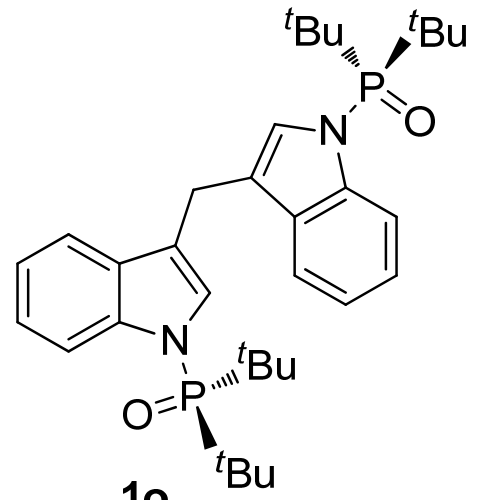

${ }^{13} \mathrm{C}$ NMR

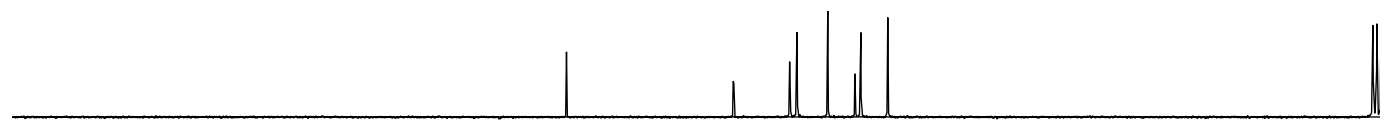

$\begin{array}{llllllllllllllllllllllll}185 & 180 & 175 & 170 & 165 & 160 & 155 & 150 & 145 & 140 & 135 & 130 & 125 & 120 & 115 & 110 & 105 & 100 & 95 & 90 & 85\end{array}$ 
$\overrightarrow{0}$
i
1

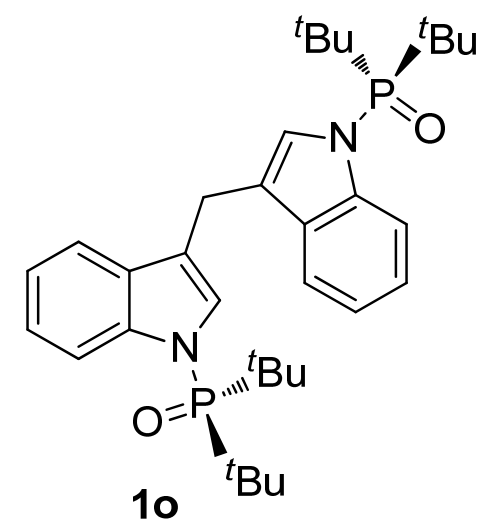

${ }^{31} \mathrm{P} N \mathrm{NMR}$ 
9. ${ }^{1} \mathrm{H},{ }^{13} \mathrm{C},{ }^{31} \mathrm{P}$ and ${ }^{19} \mathrm{~F}$ NMR Spectra of Products

कo
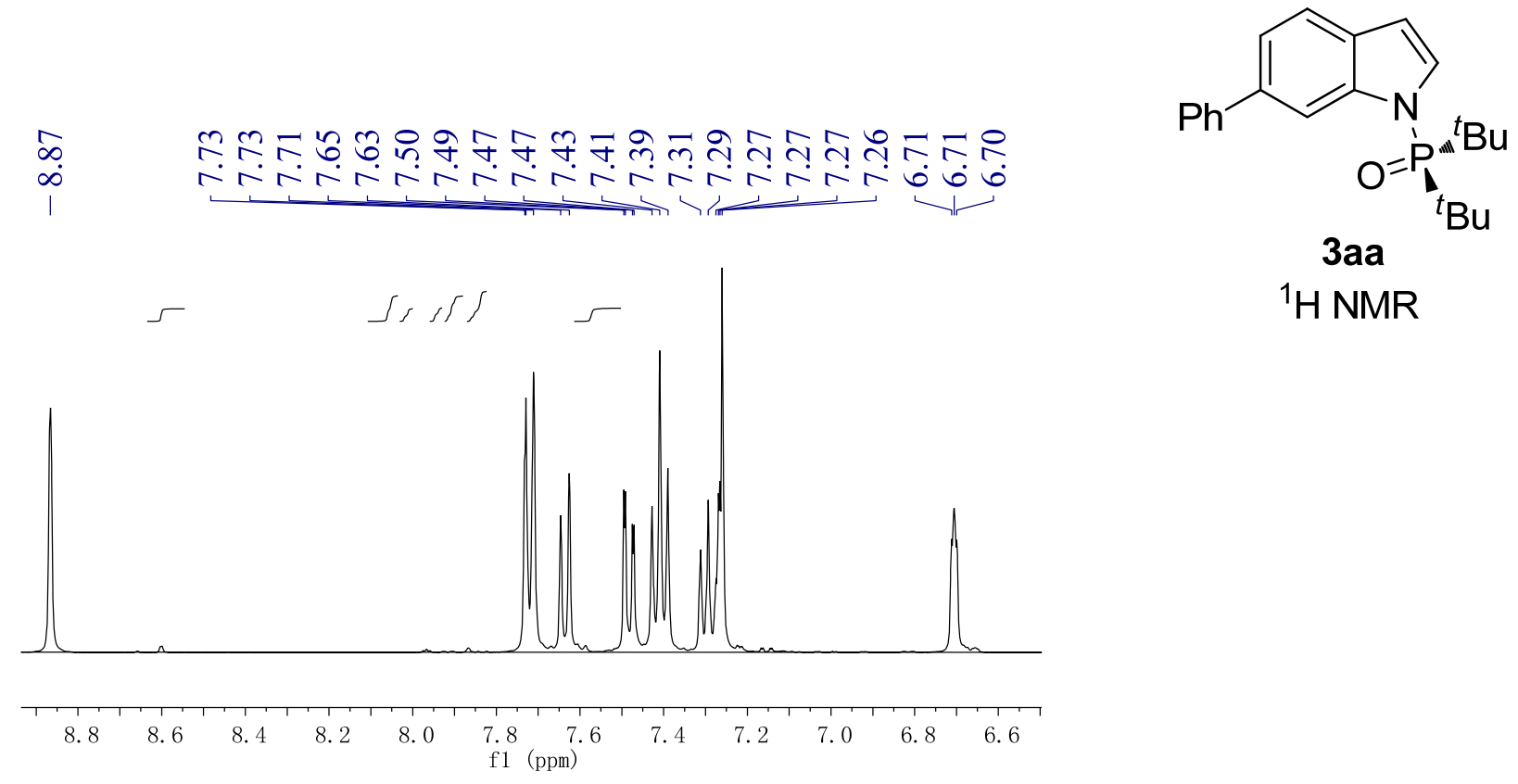

3aa

${ }^{1} \mathrm{H}$ NMR

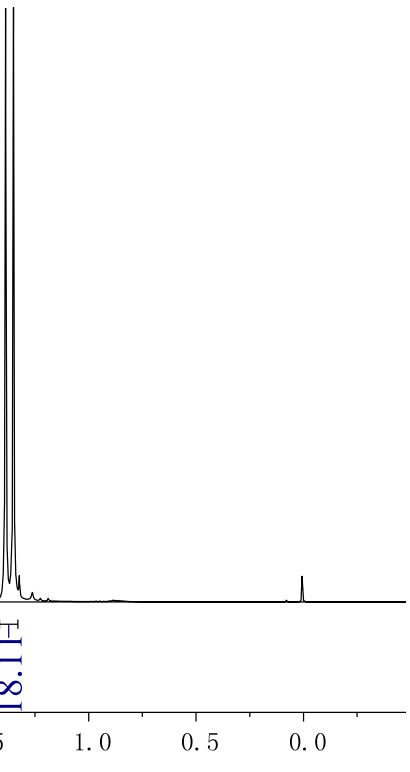




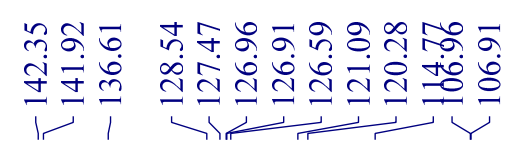

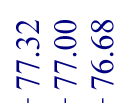

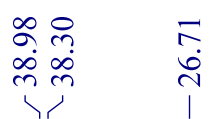

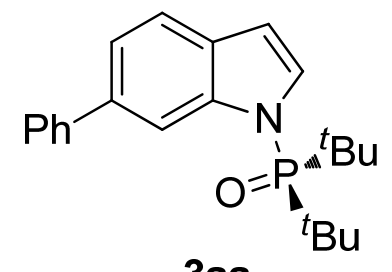

3aa

${ }^{13} \mathrm{C}$ NMR

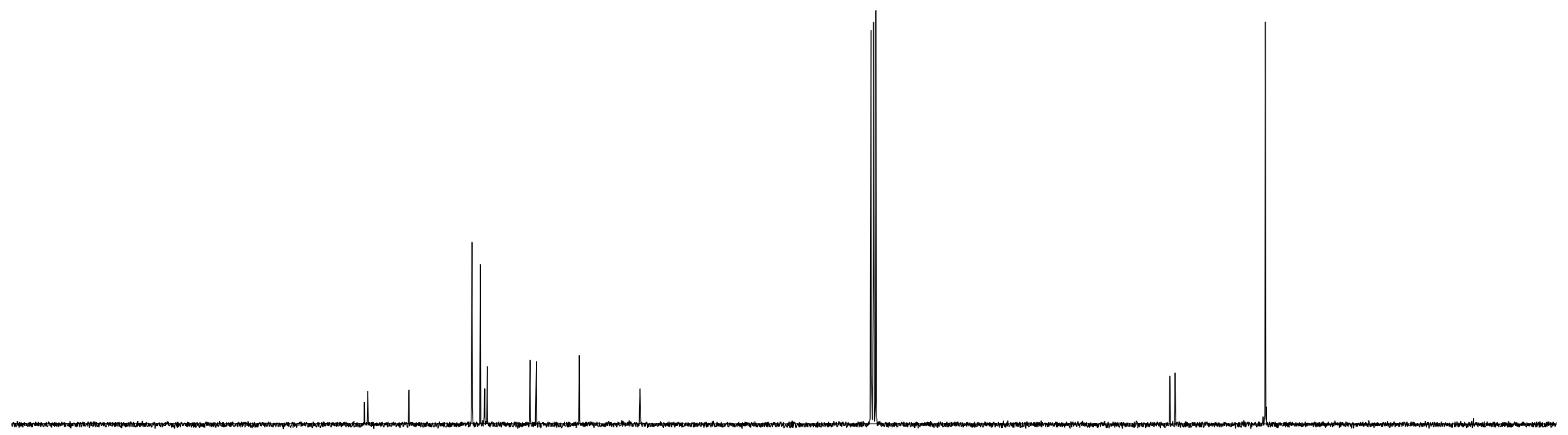

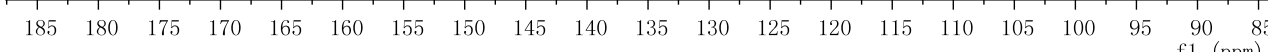


$\frac{0}{30}$
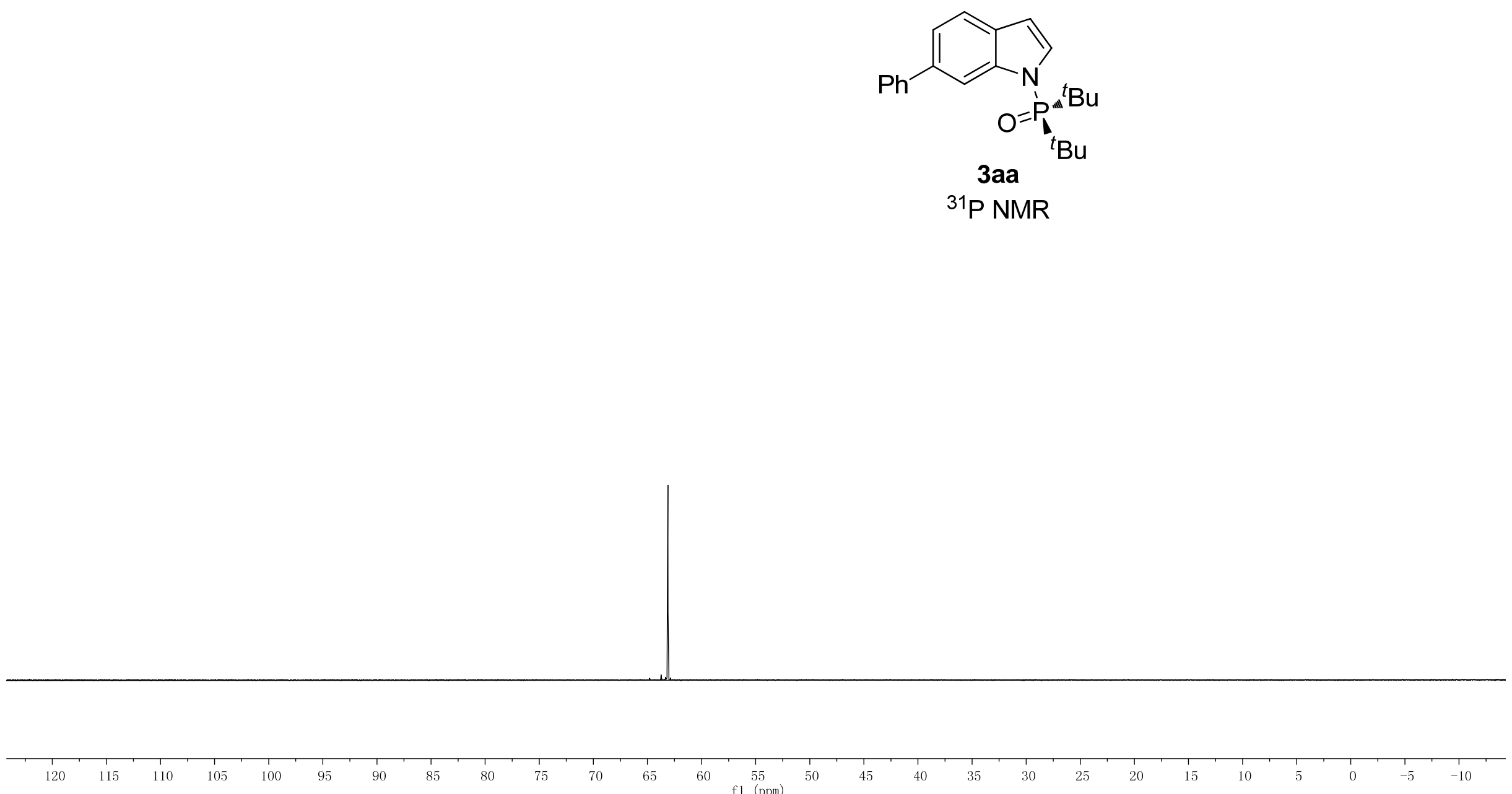


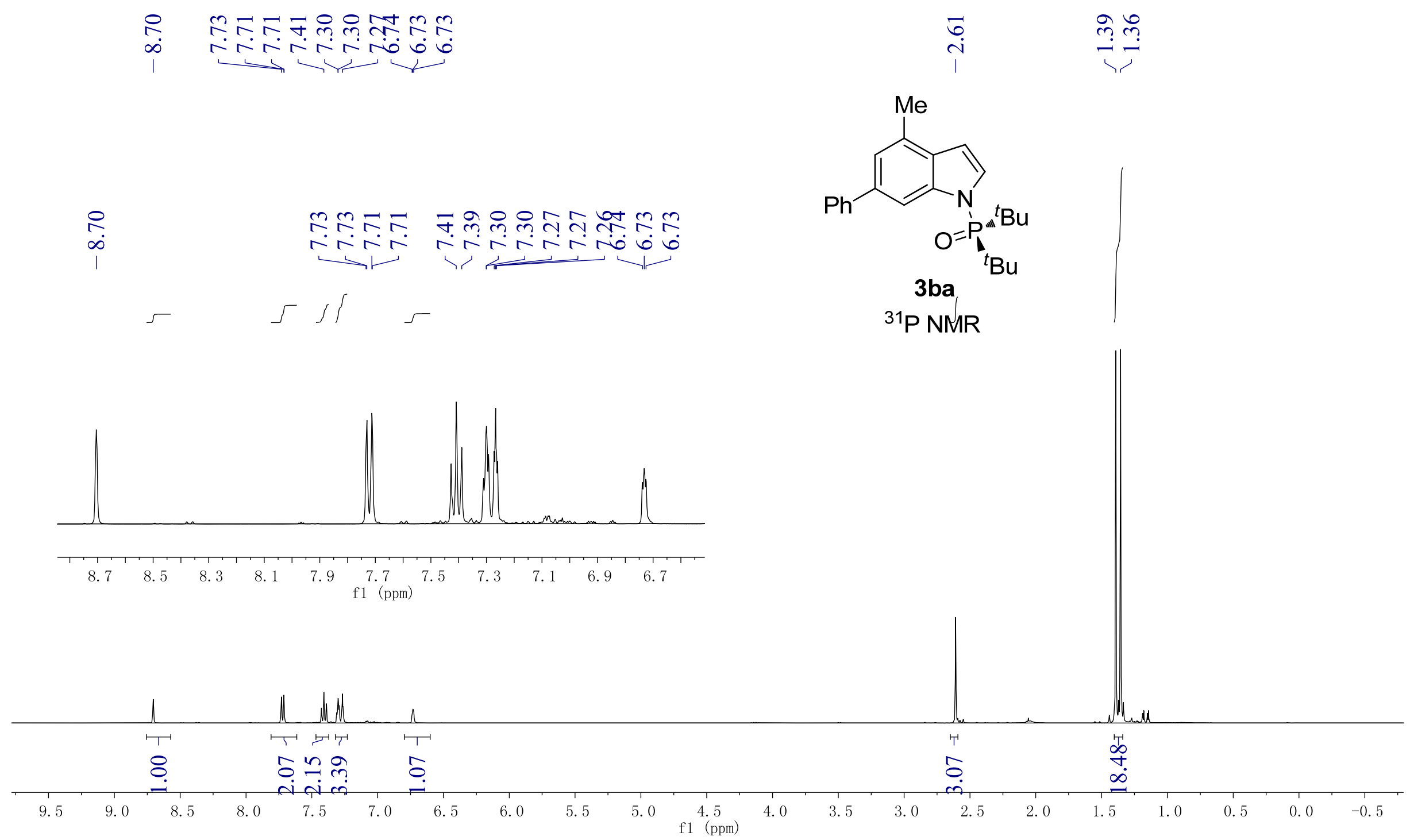




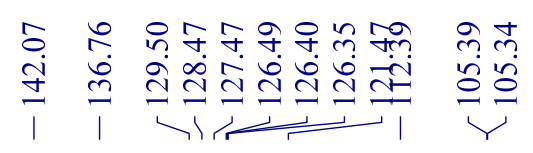

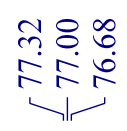

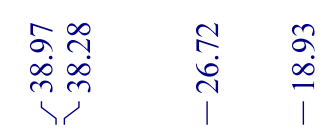

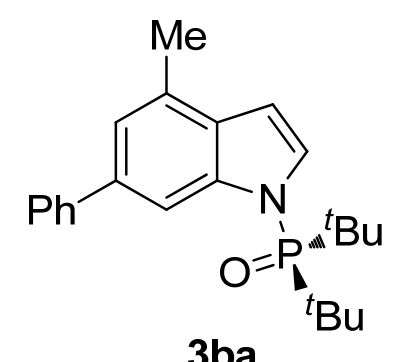

${ }^{13} \mathrm{C}$ NMR

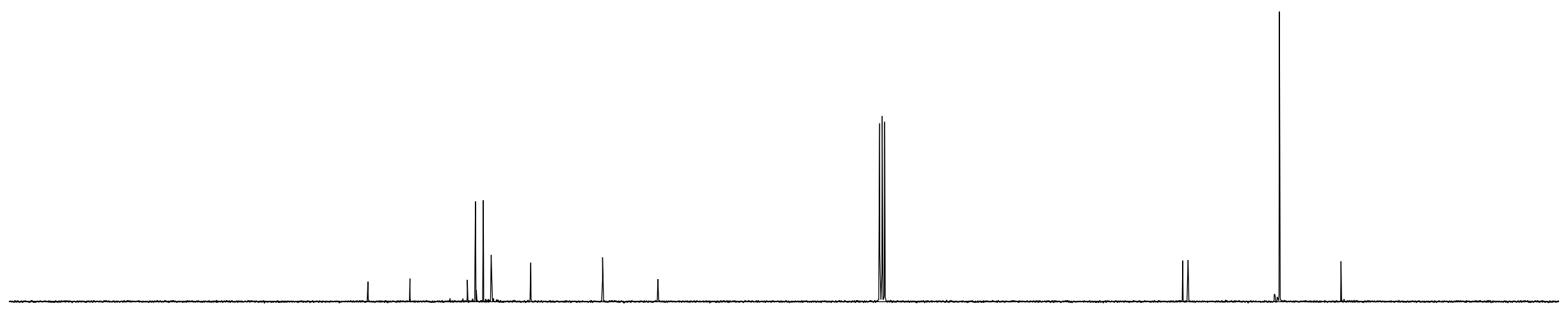

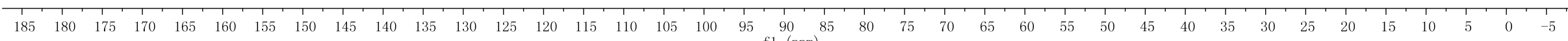


के

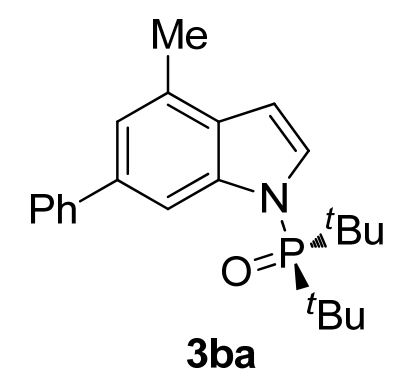

${ }^{31} \mathrm{P}$ NMR

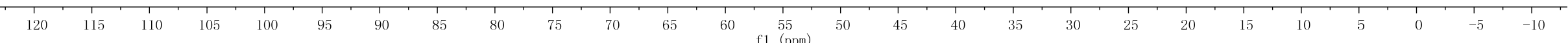




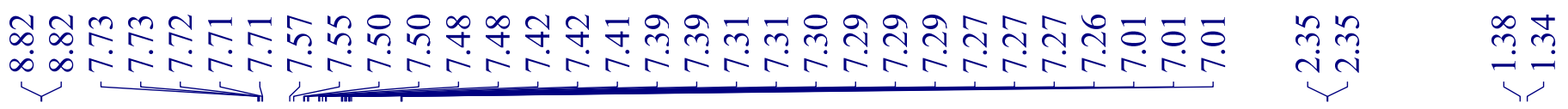
o on

Jifles
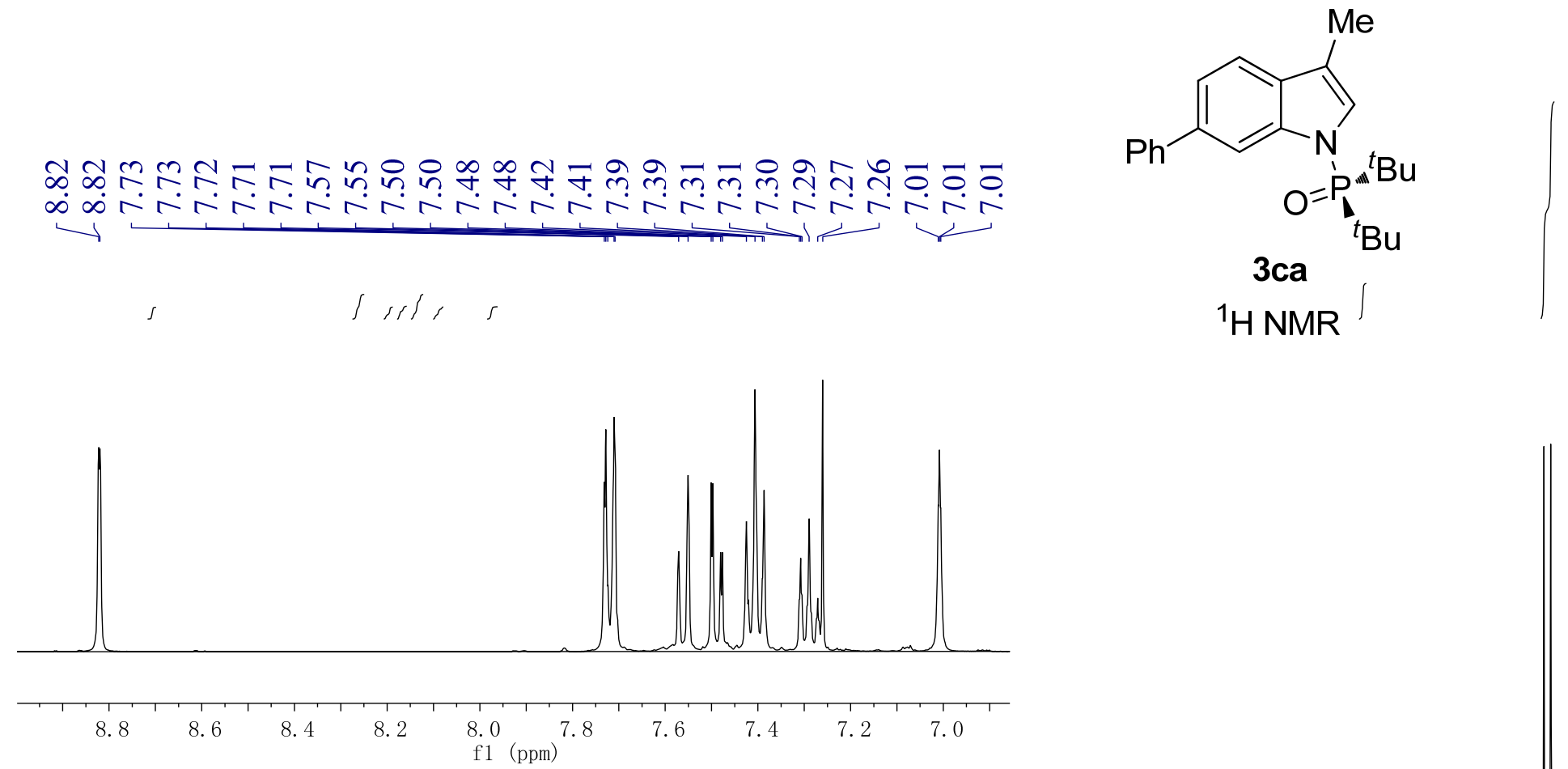

H NMR

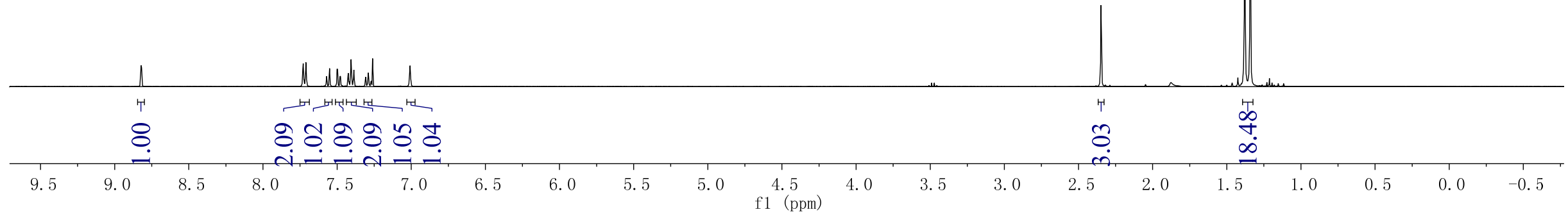




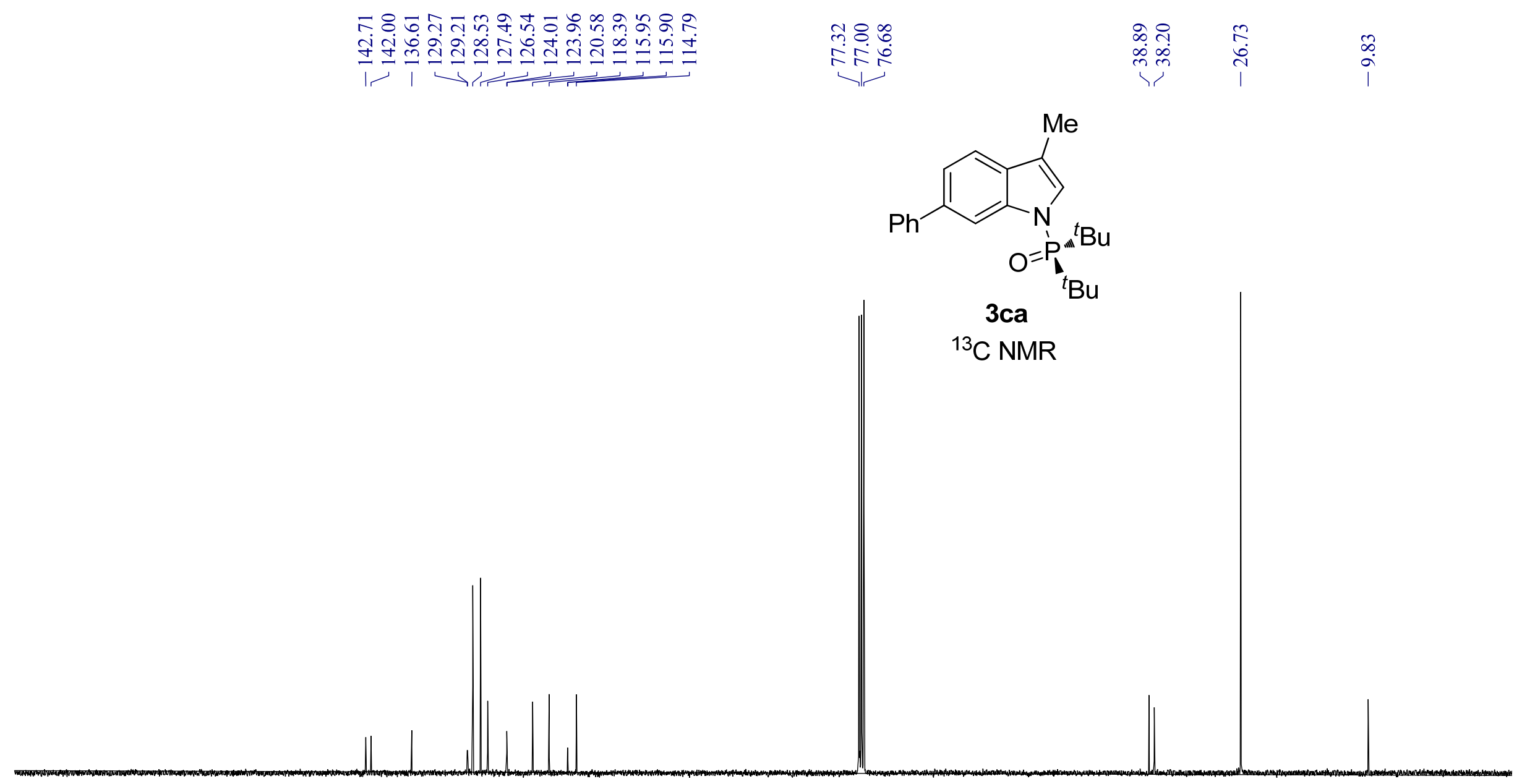

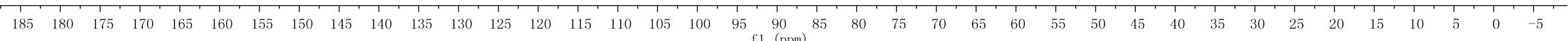




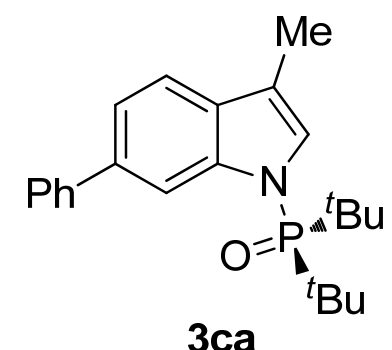

${ }^{31} \mathrm{P}$ NMR

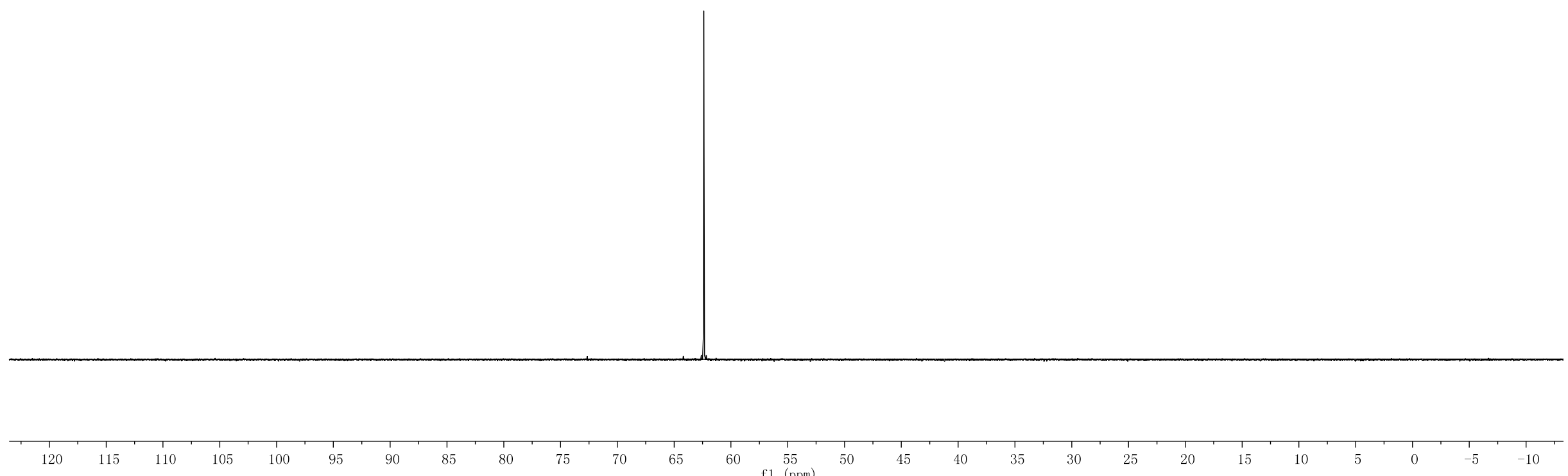




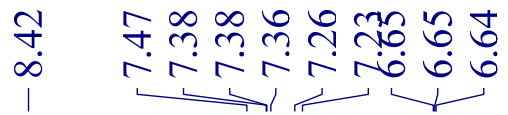

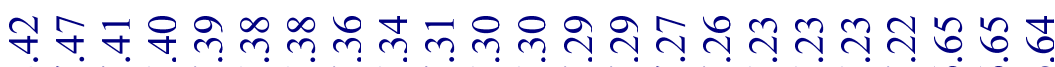

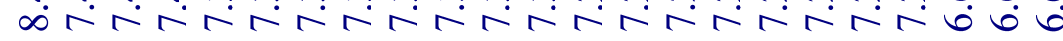
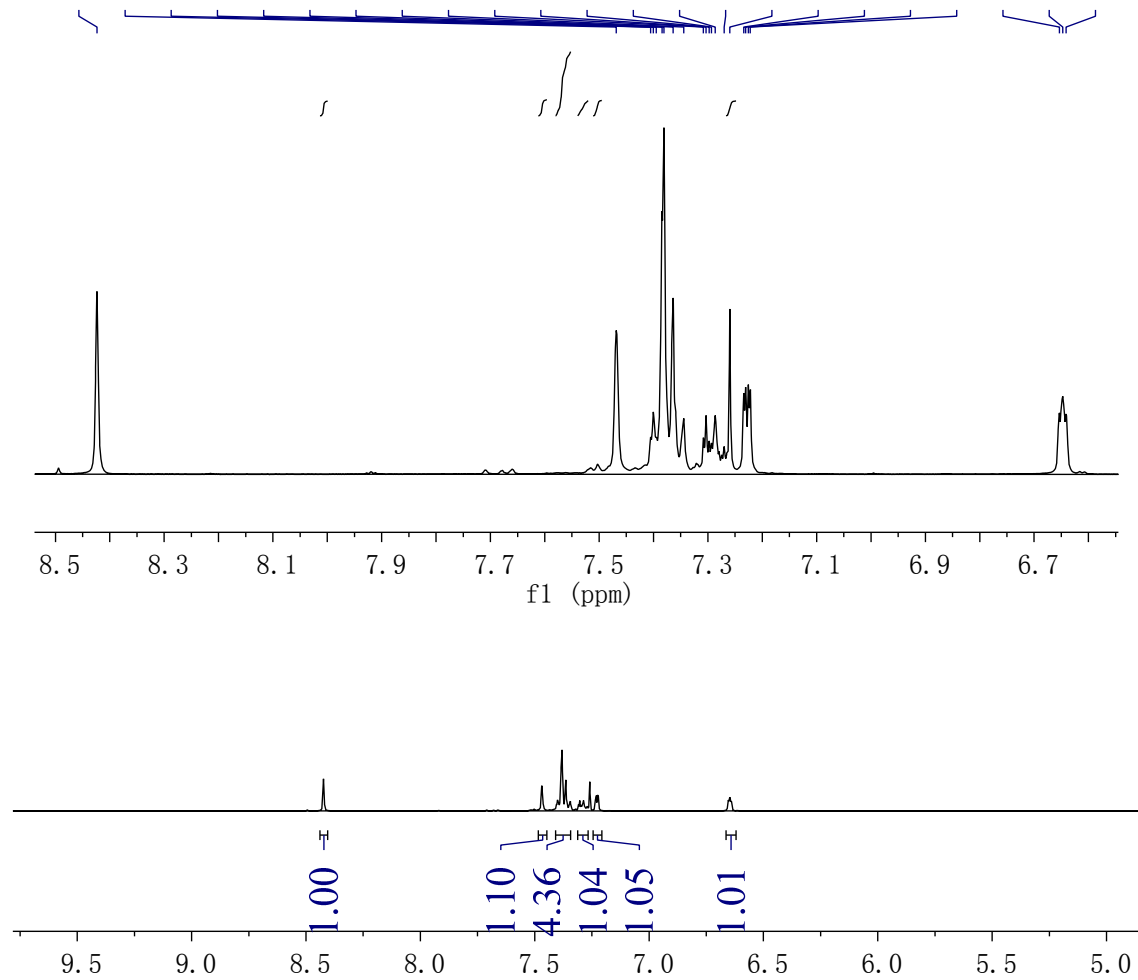
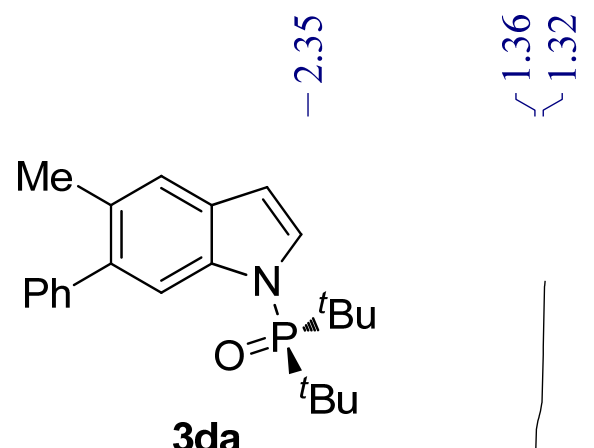

${ }^{1} \mathrm{H}$ NMR
กุ?

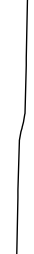

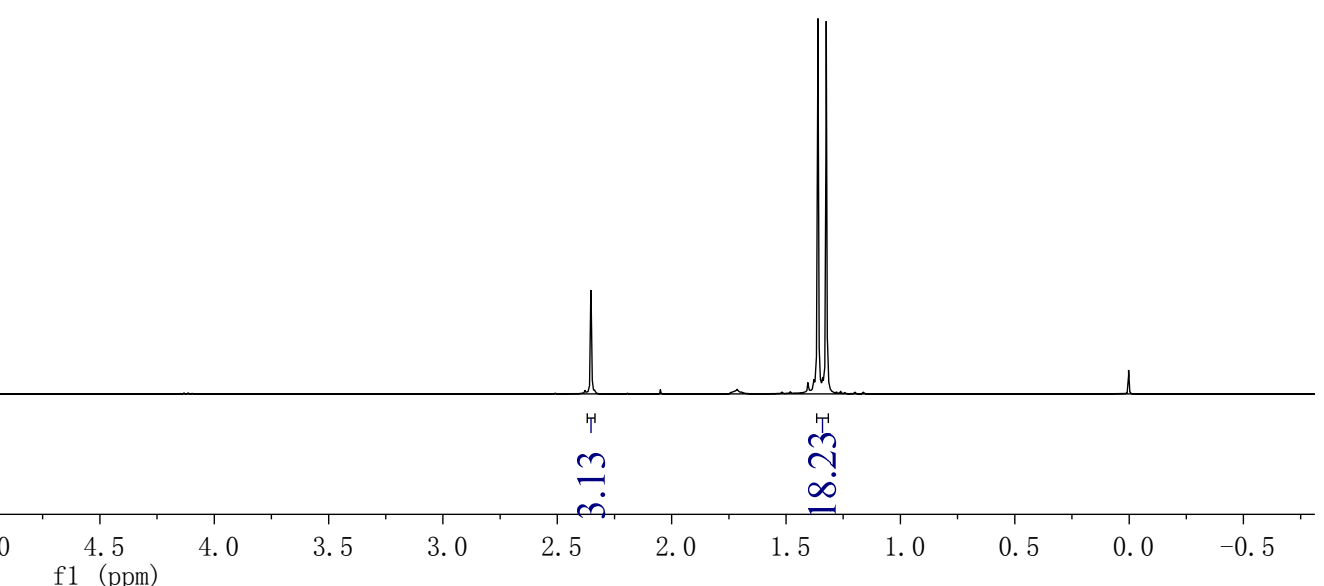




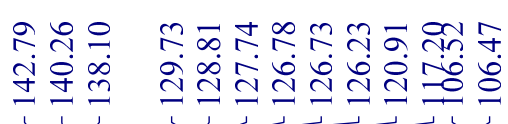

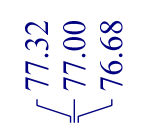

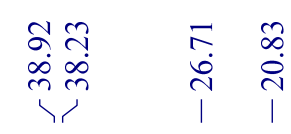

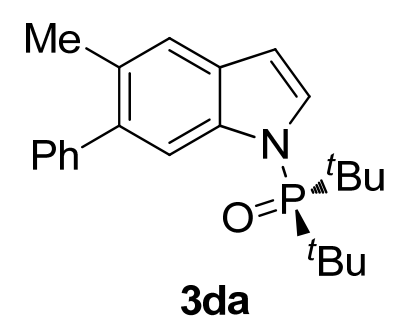

${ }^{13} \mathrm{C}$ NMR

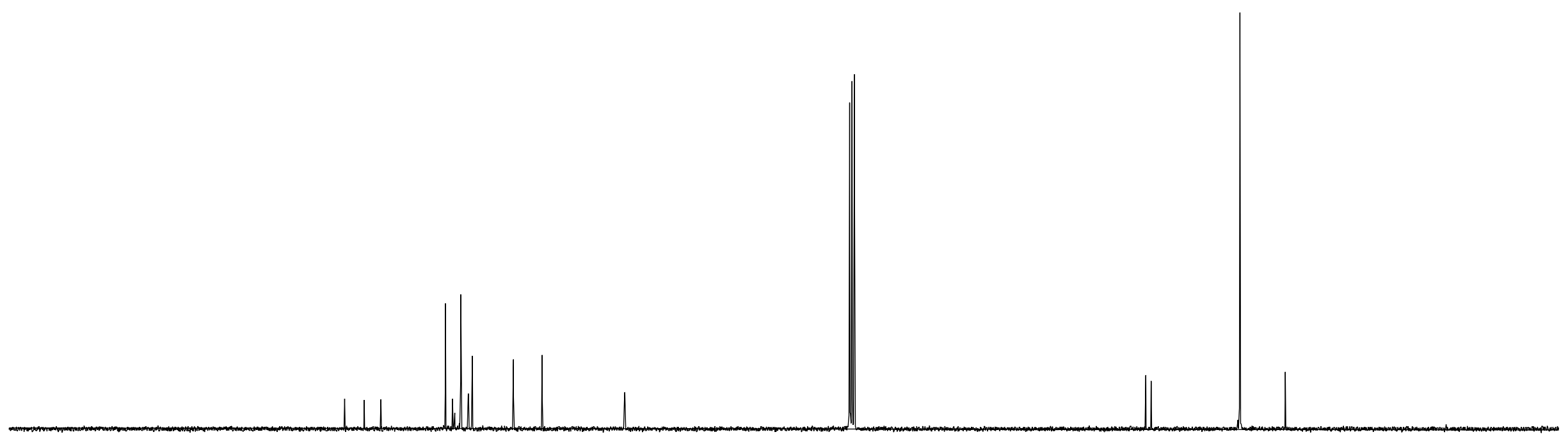

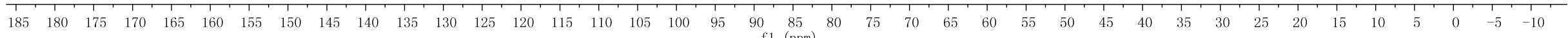


i্
i
1

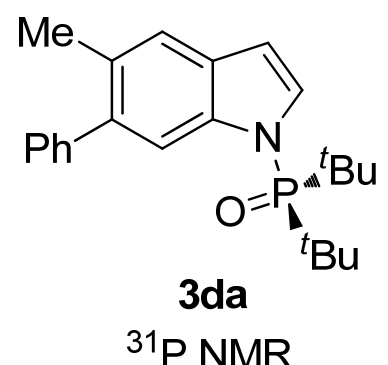

${ }^{31} \mathrm{P}$ NMR

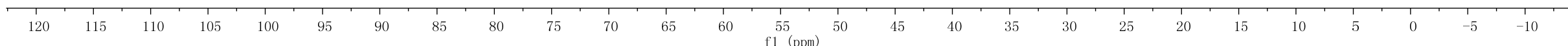




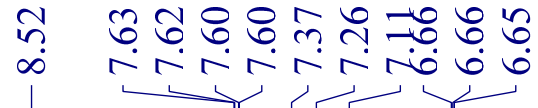
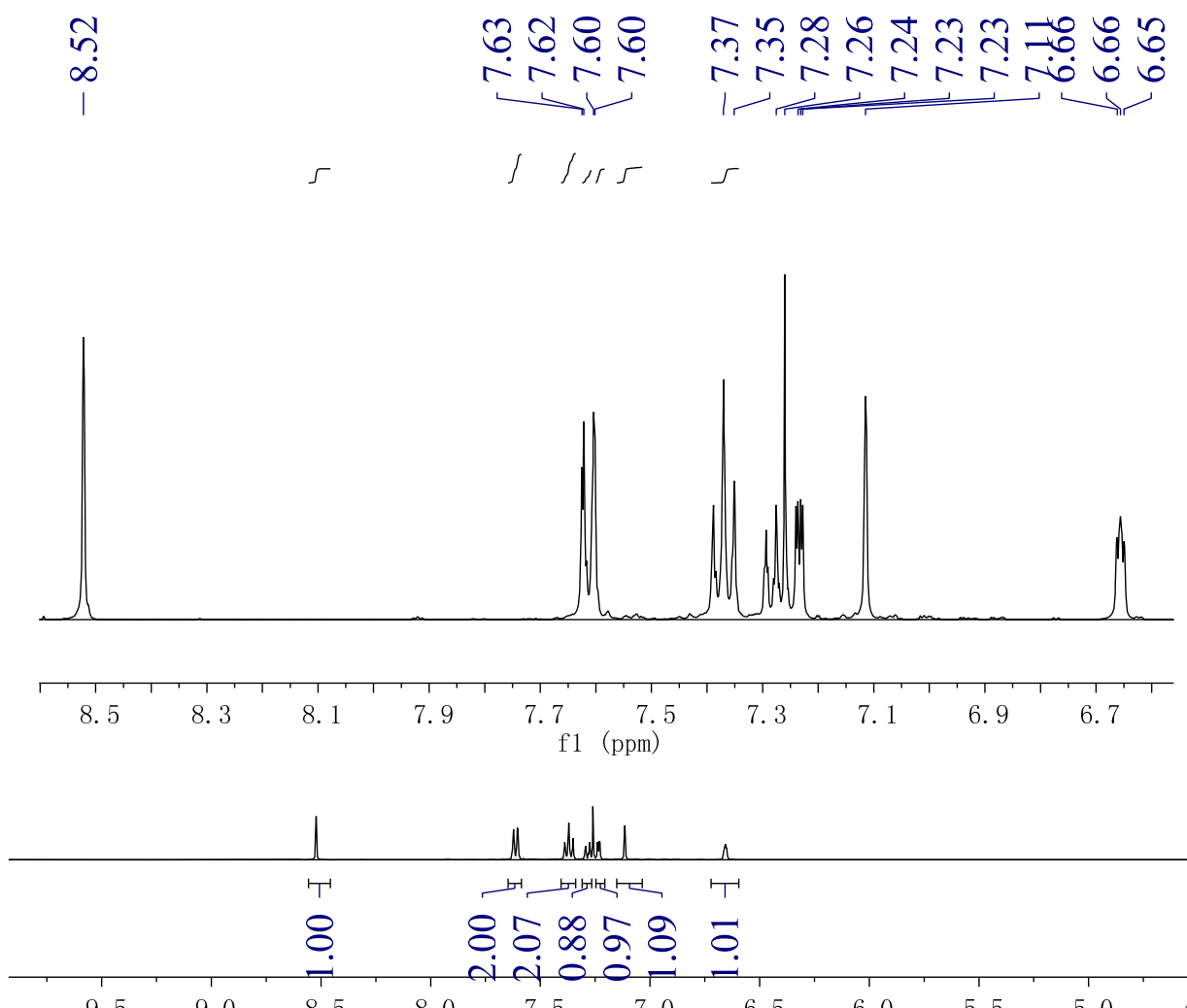

9.5 $\infty$
$\infty$

ํํㄹำำ

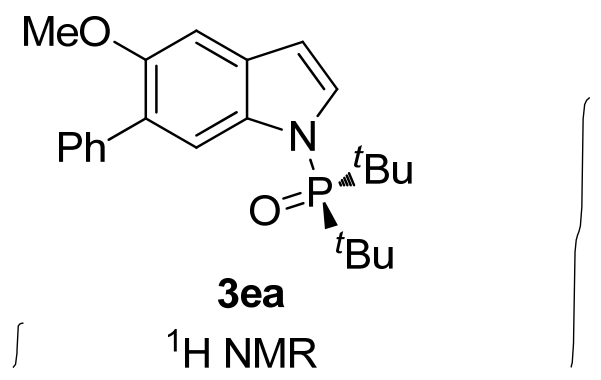




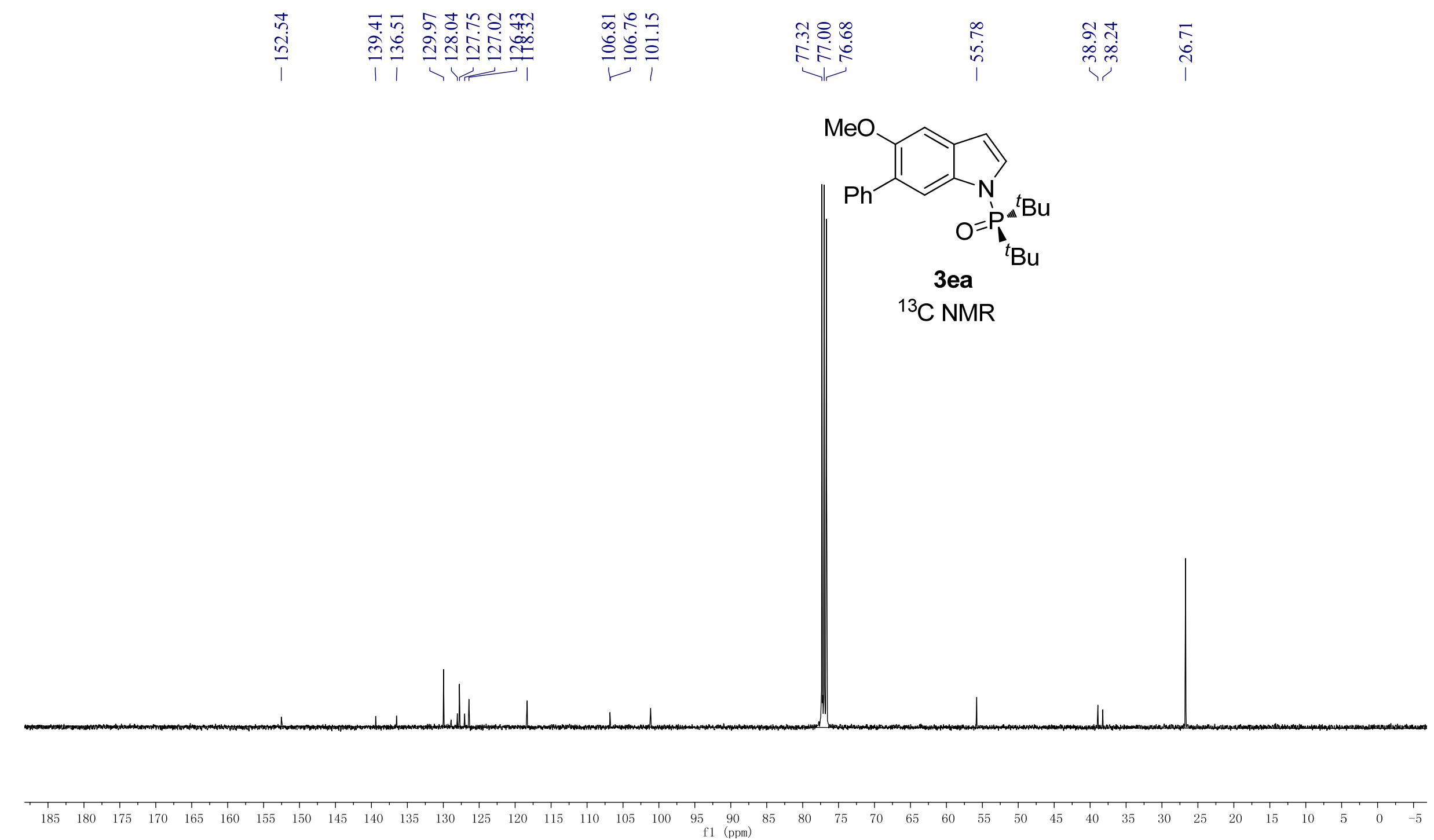




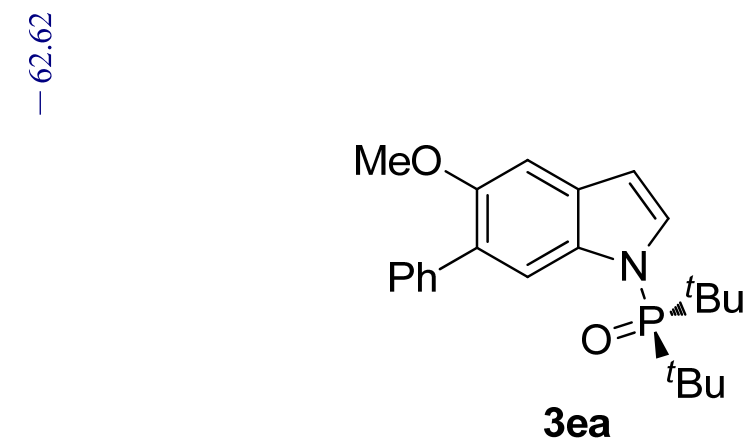

${ }^{31} \mathrm{P}$ NMR

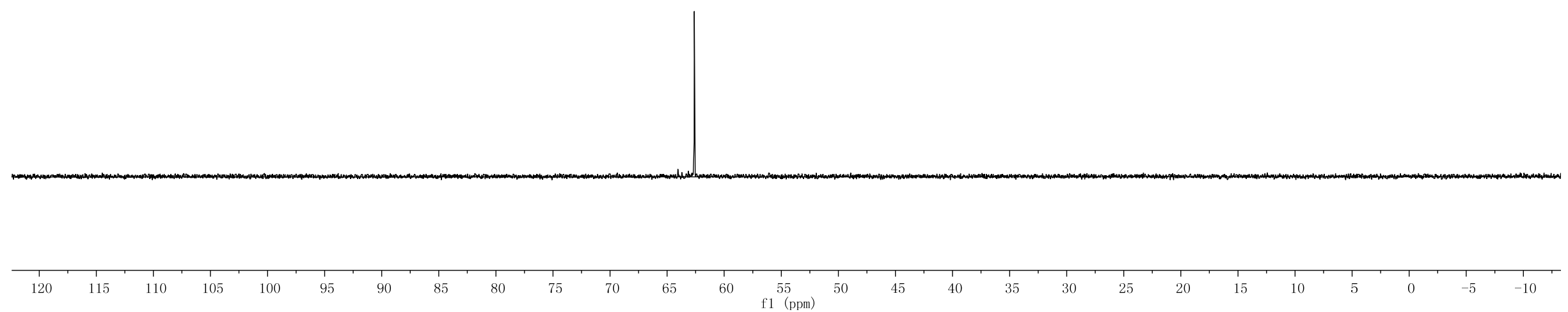




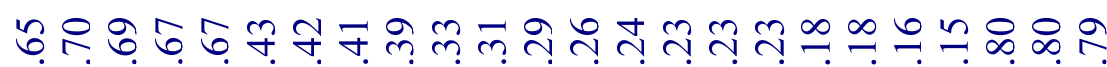

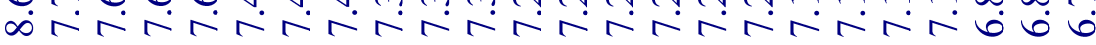

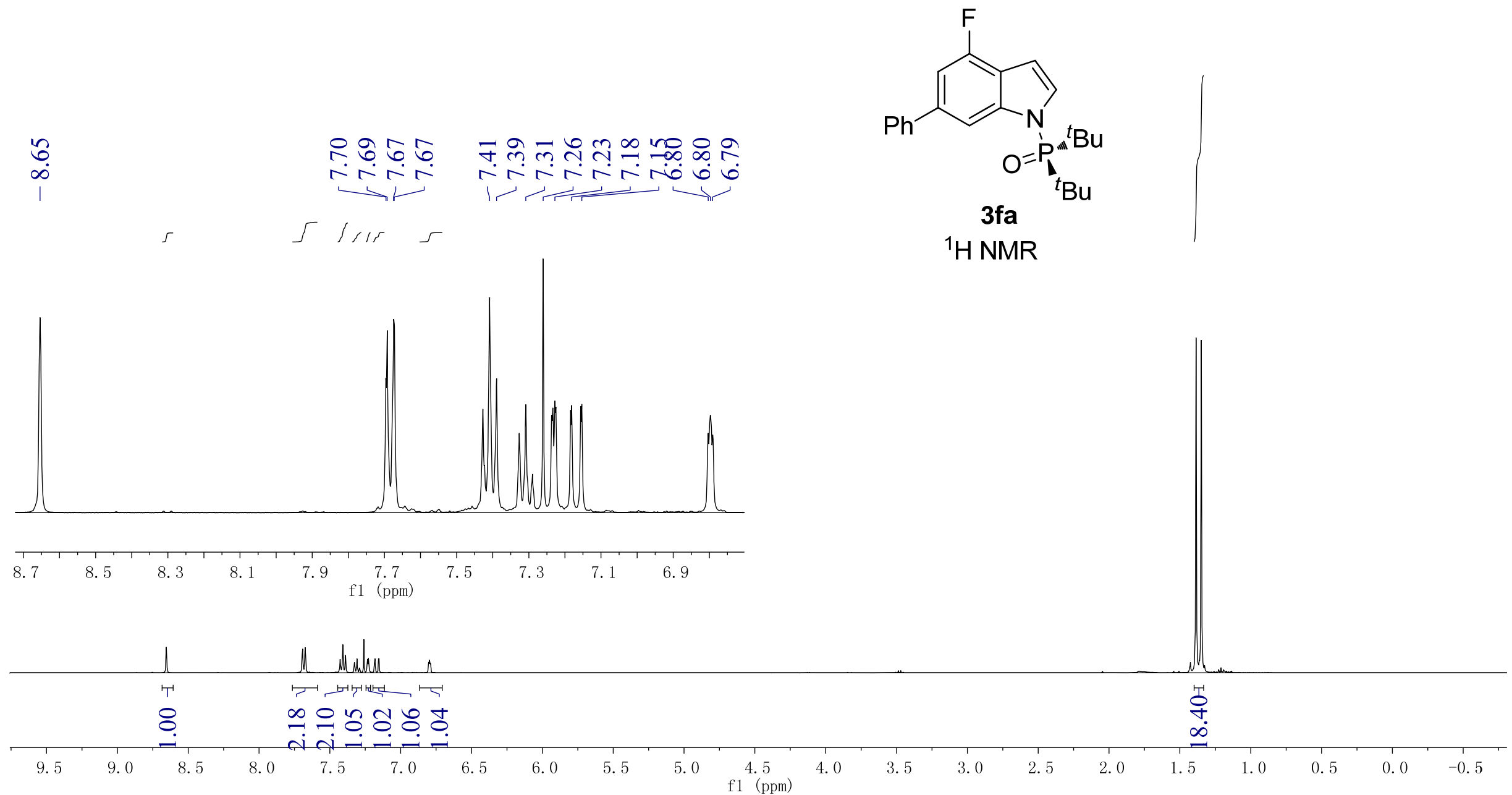



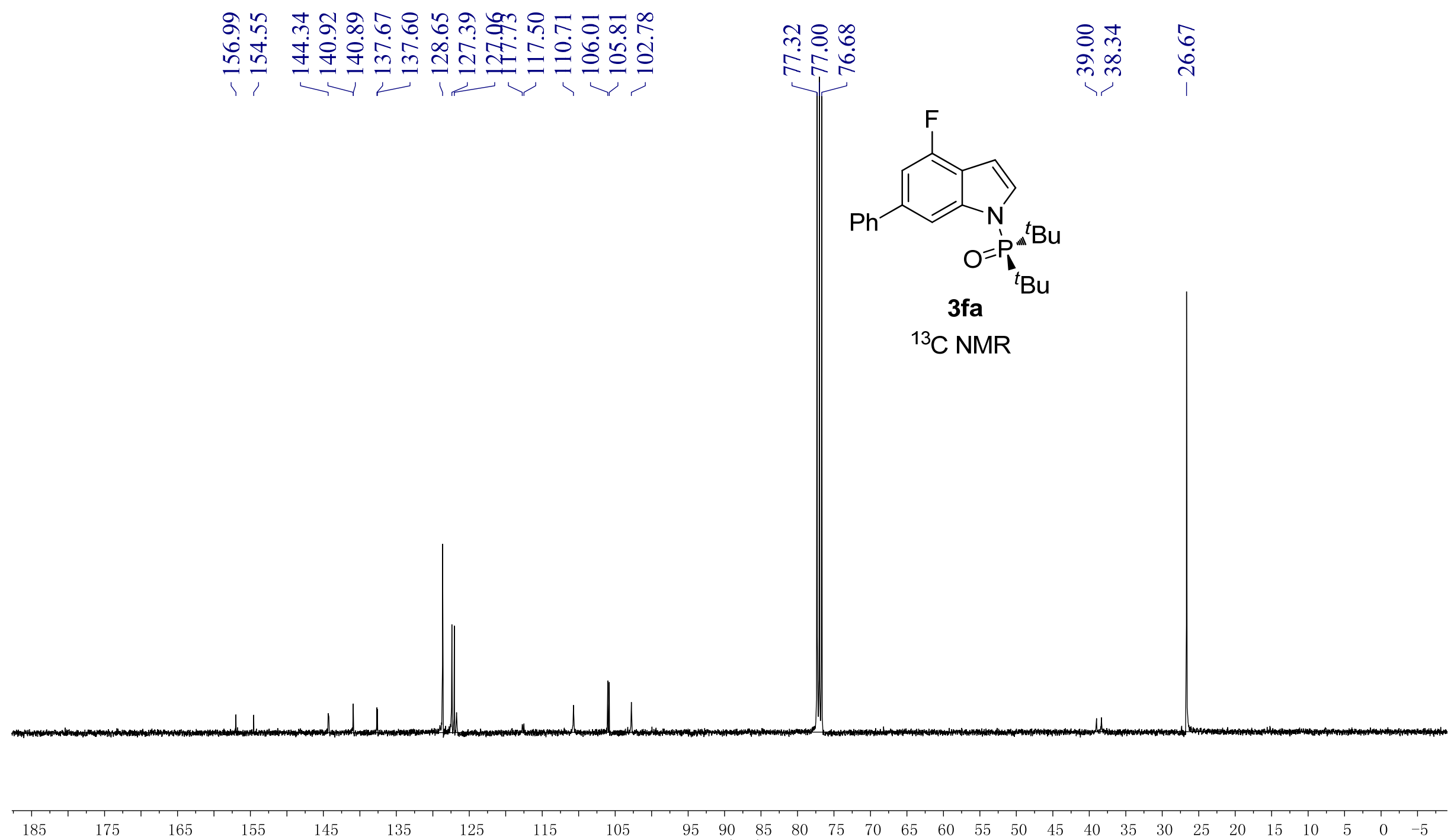
वे
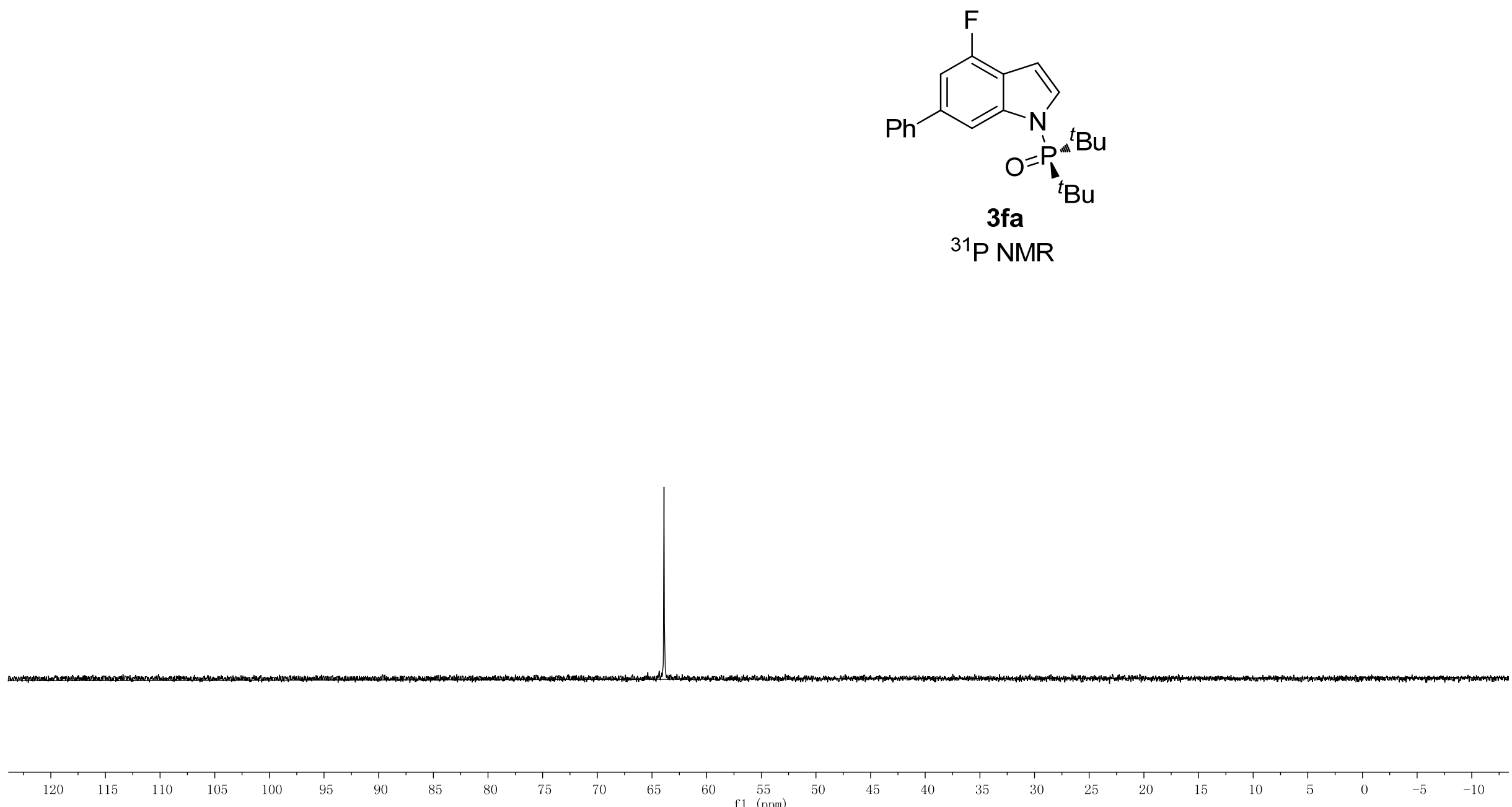


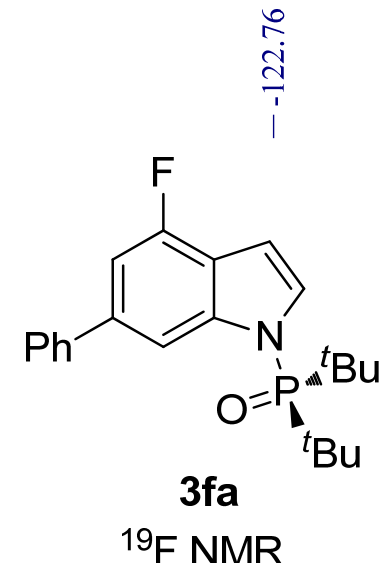

${ }^{19} \mathrm{~F}$ NMR 


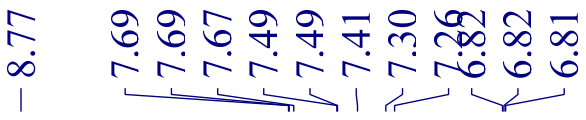

कृ m

र

$$
\underset{\substack{n \\ \infty \\ 1}}{\substack{0 \\ 0}}
$$$$
\text { बे }
$$

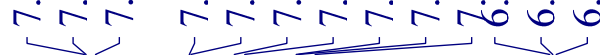
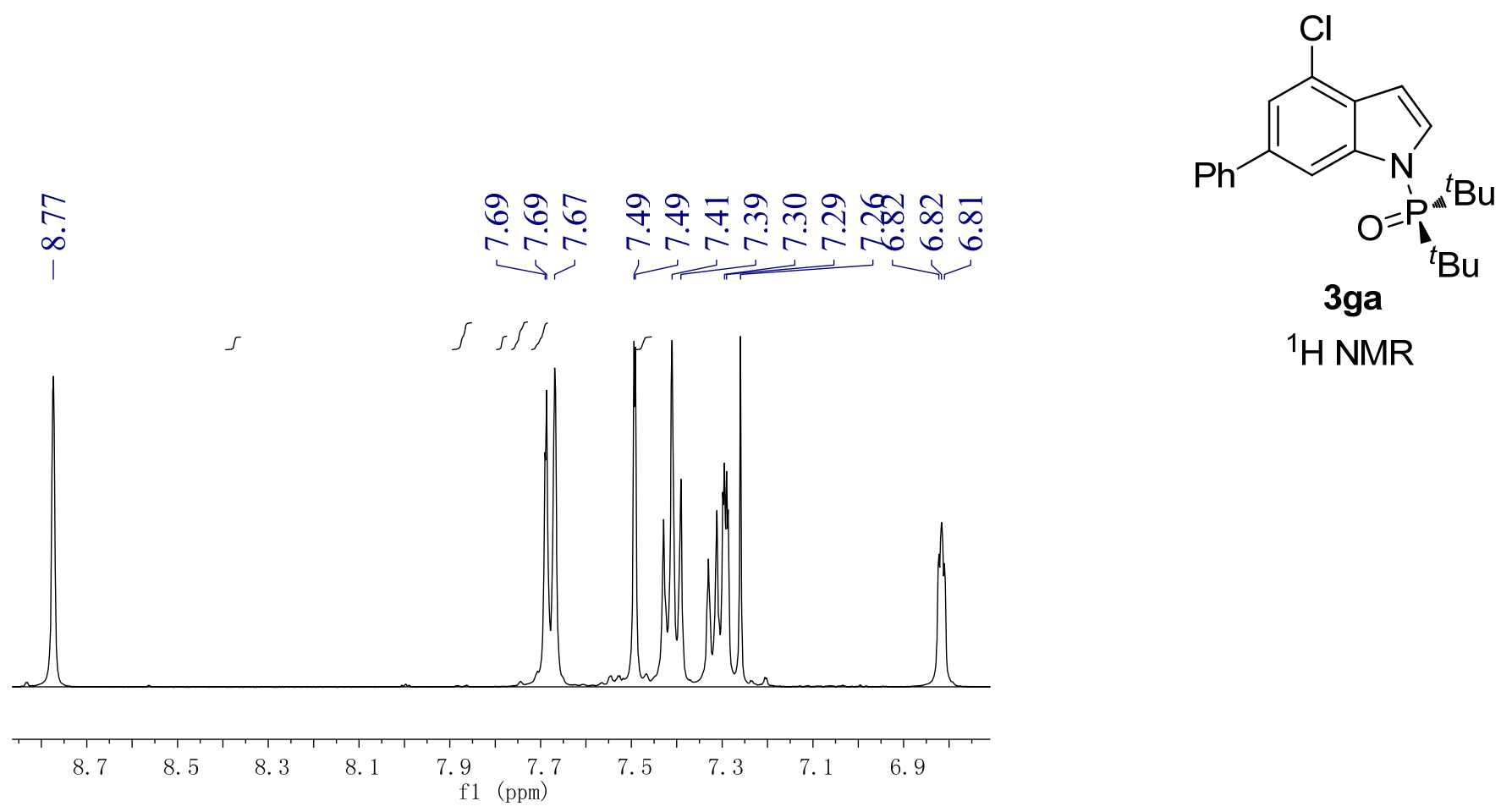

$3 g a$

${ }^{1} \mathrm{H}$ NMR

\section{$1|M|$}

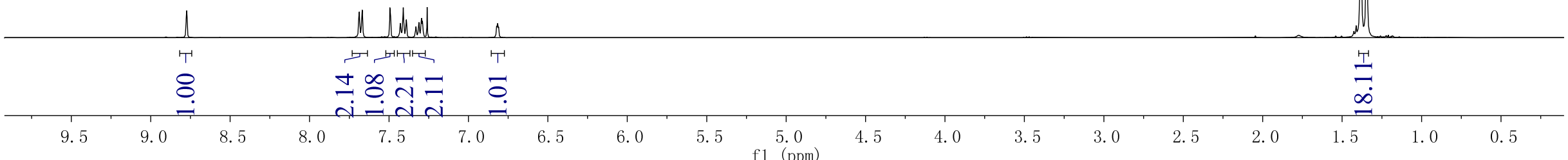




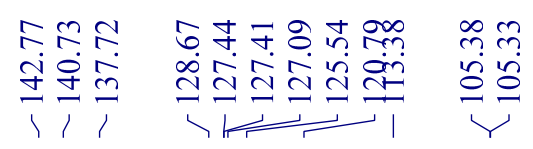

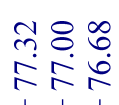

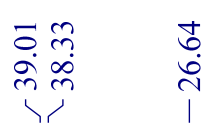

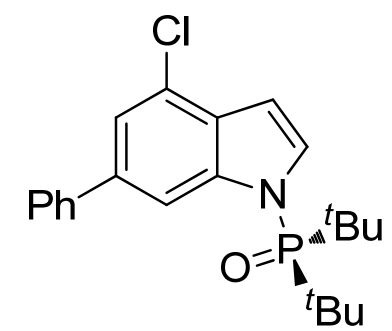

3ga

${ }^{13} \mathrm{C}$ NMR

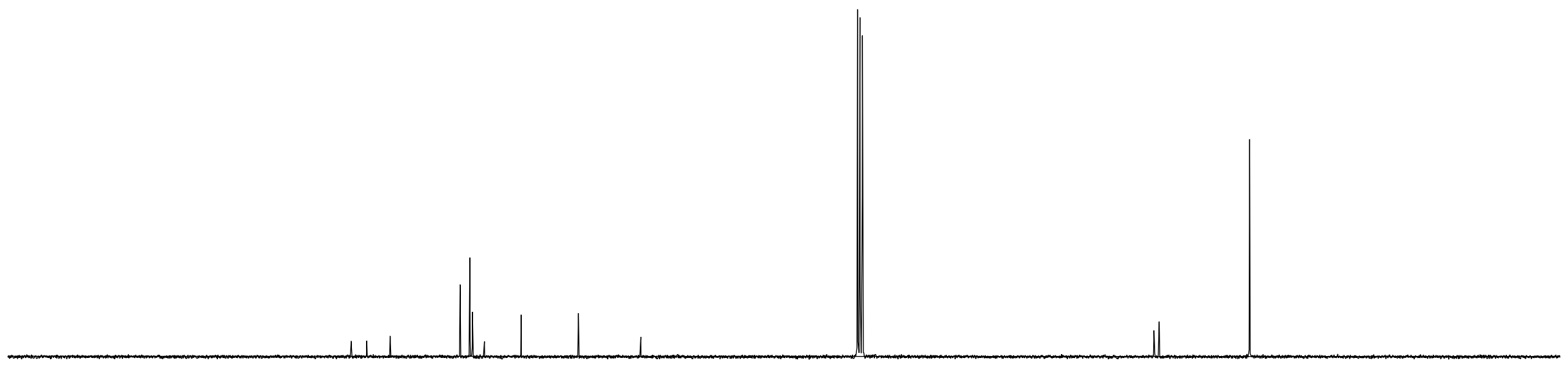

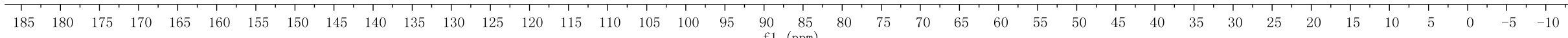




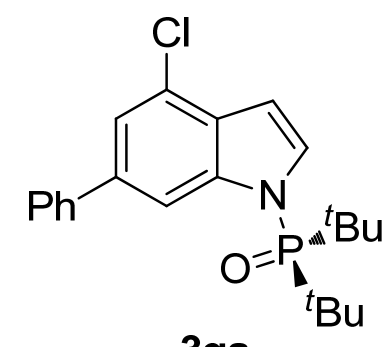

$3 g a$

${ }^{31} \mathrm{P}$ NMR

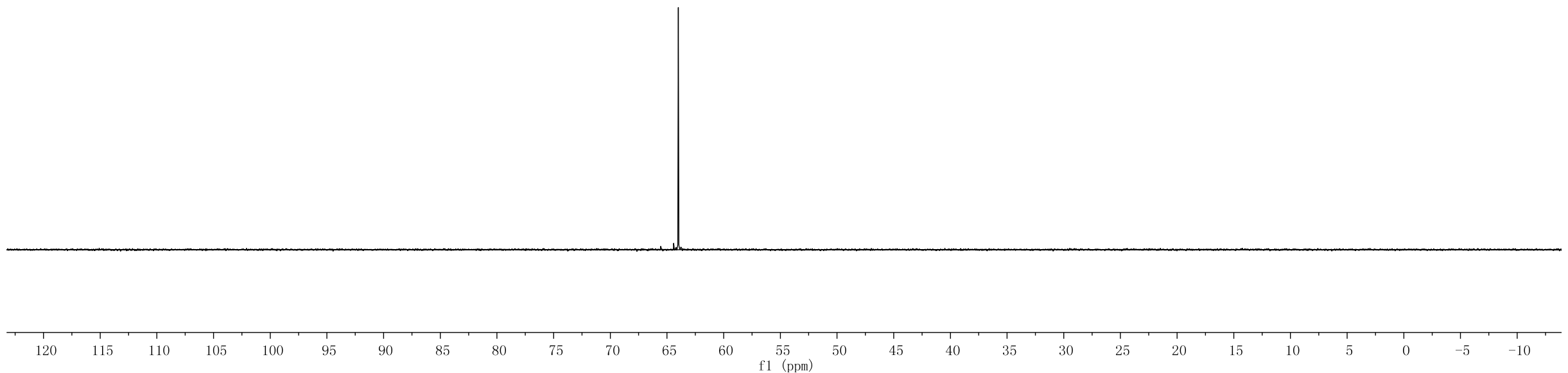


ڤ<smiles>CC(C)(C)CP(=O)(n1cc(Cl)c2ccc(-c3ccccc3)cc21)C(C)(C)C</smiles>

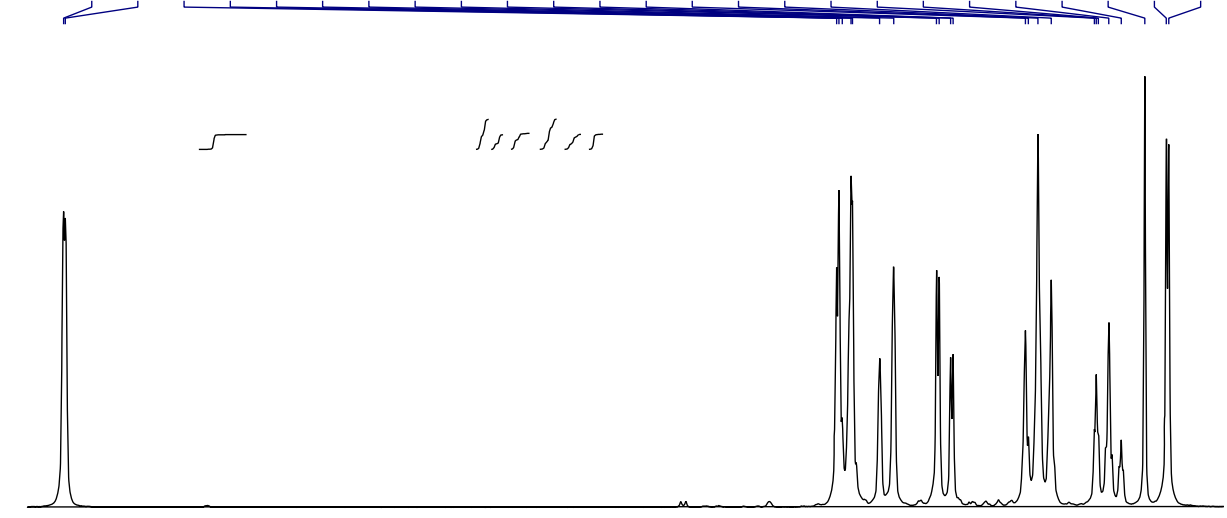

3ha

${ }^{1} \mathrm{H}$ NMR
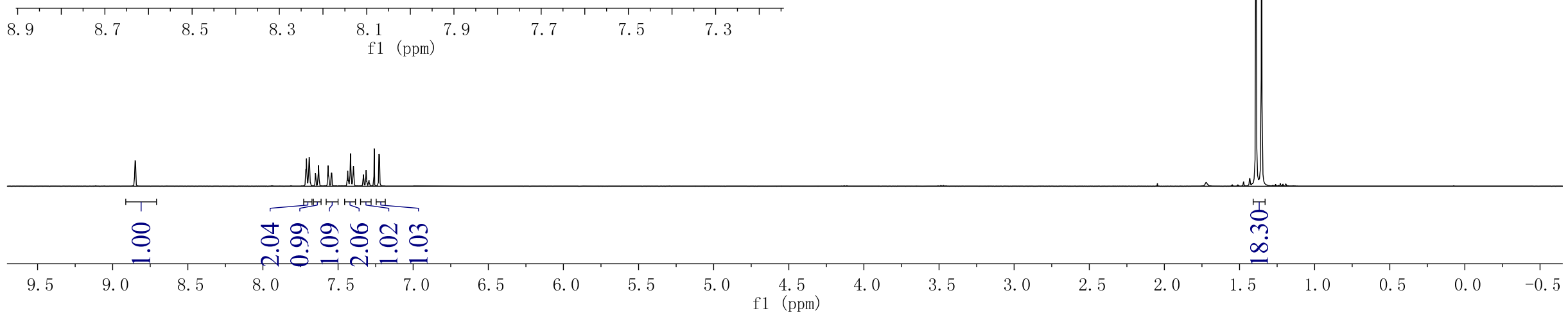

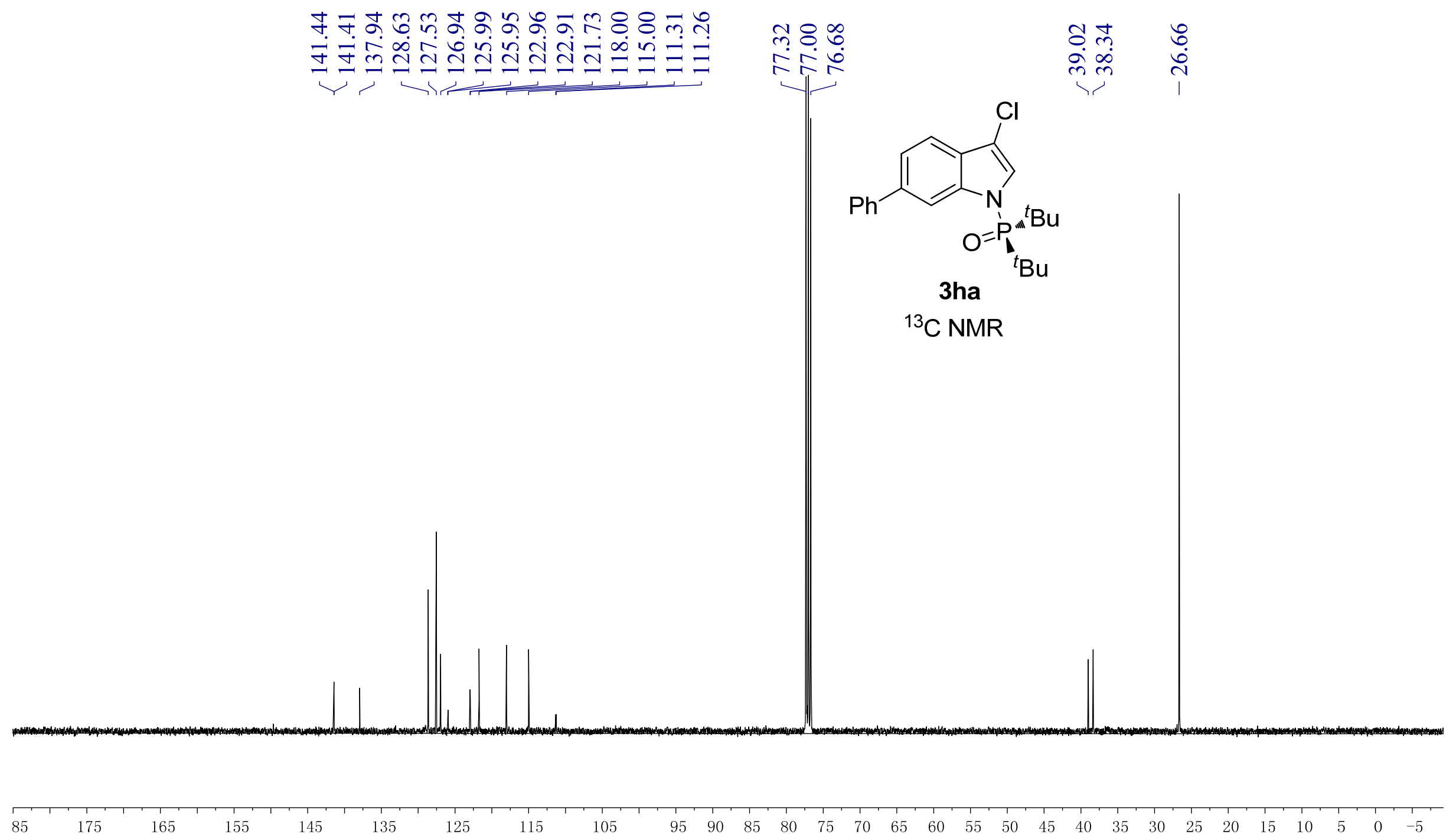

$\mathrm{f1}$ (ppm) 


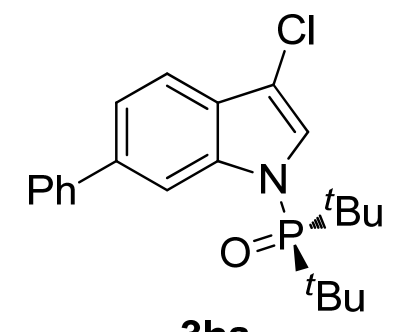

3ha

${ }^{31} \mathrm{P}$ NMR

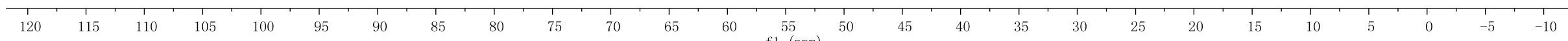




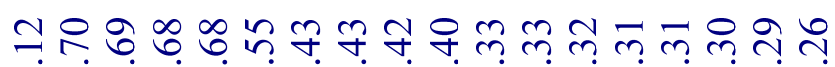

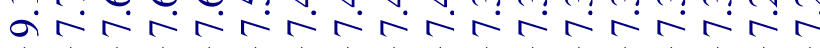

守 宅

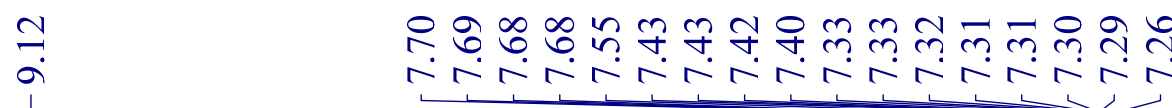

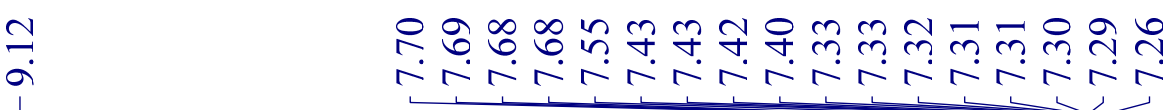

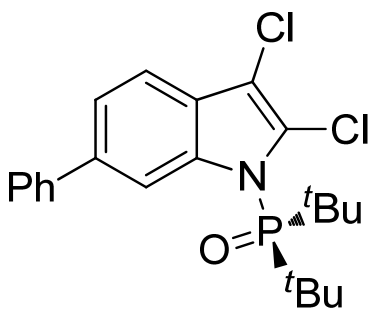

$\iiint S$

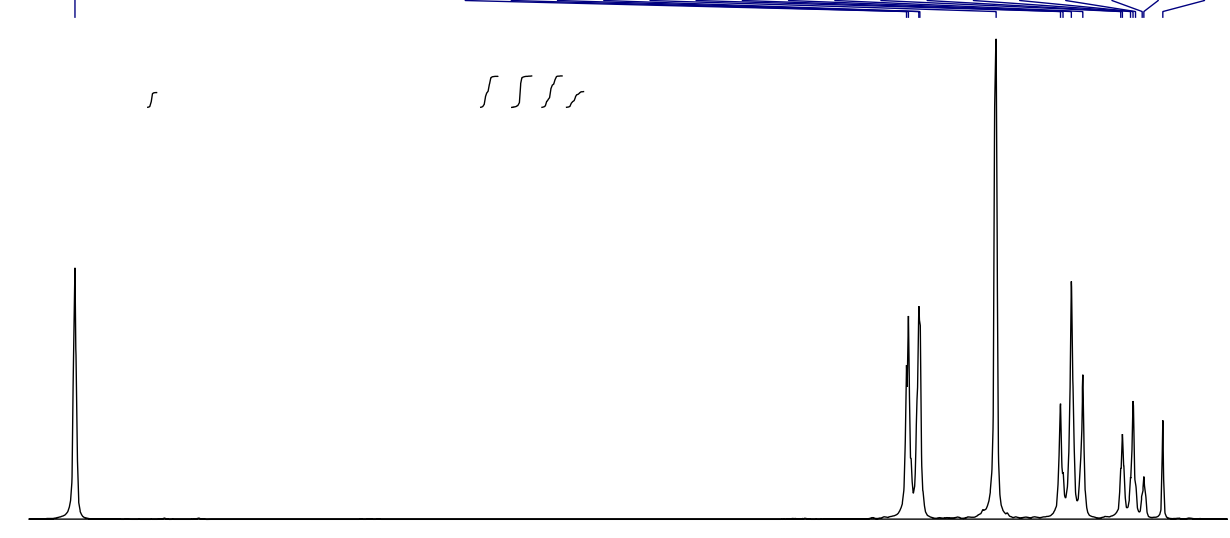

3ia

${ }^{1} \mathrm{H}$ NMR
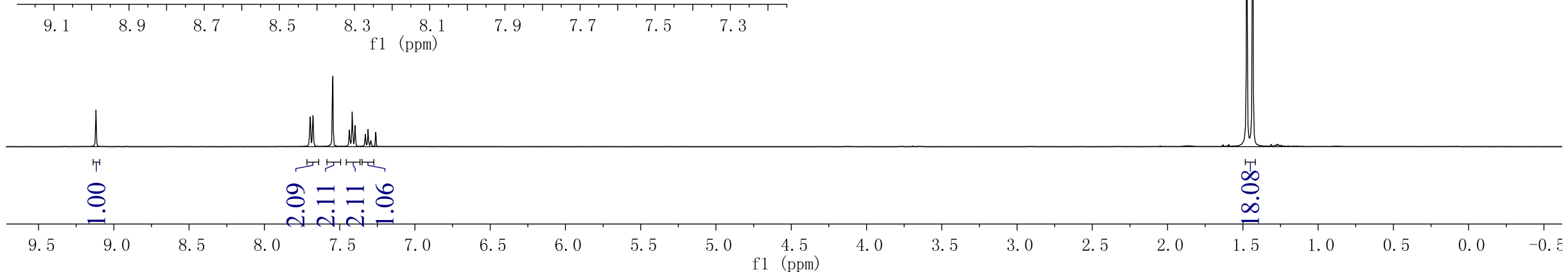


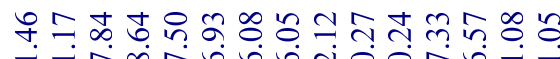

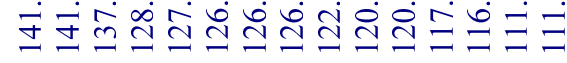

, ?

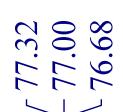

站尔

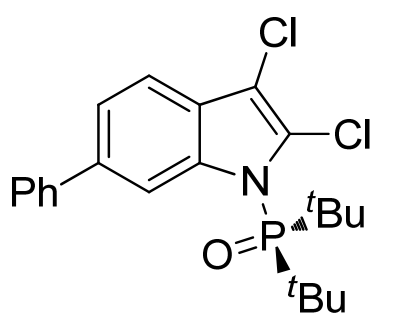

$3 i a$

${ }^{13} \mathrm{C}$ NMR

$\begin{array}{lllllllllllllllllllllllllll}185 & 180 & 175 & 170 & 165 & 160 & 155 & 150 & 145 & 140 & 135 & 130 & 125 & 120 & 115 & 110 & 105 & 100 & 95 & 90 & 85\end{array}$ 
梁

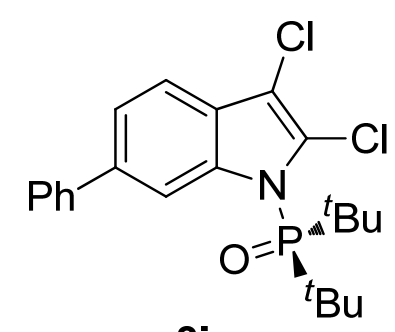

3 ia

${ }^{31} \mathrm{P}$ NMR

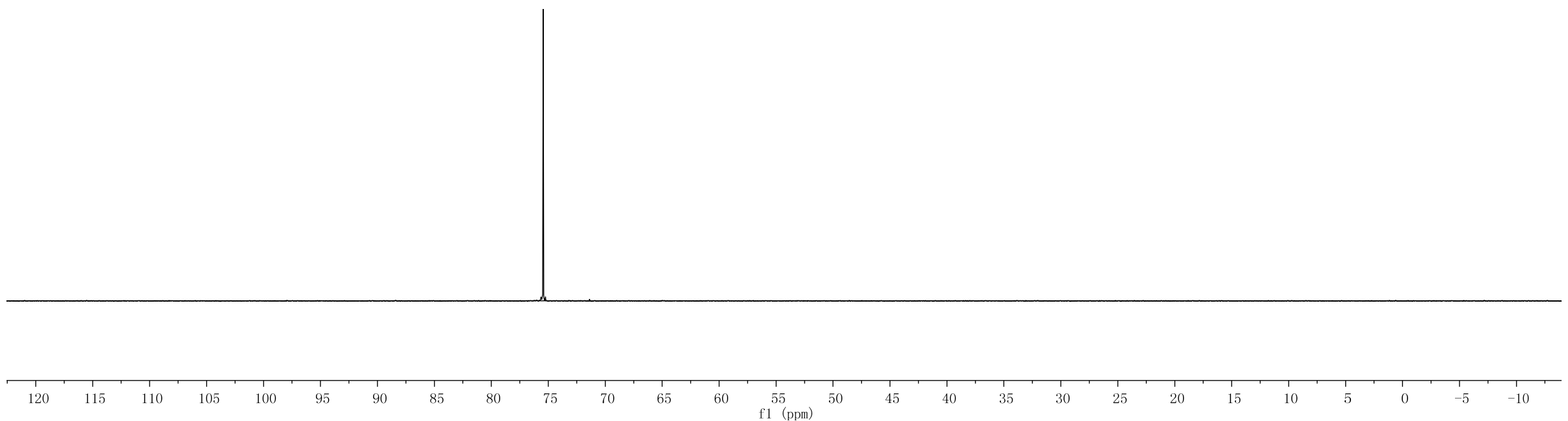


m

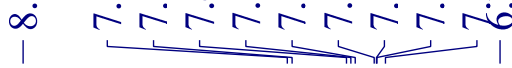
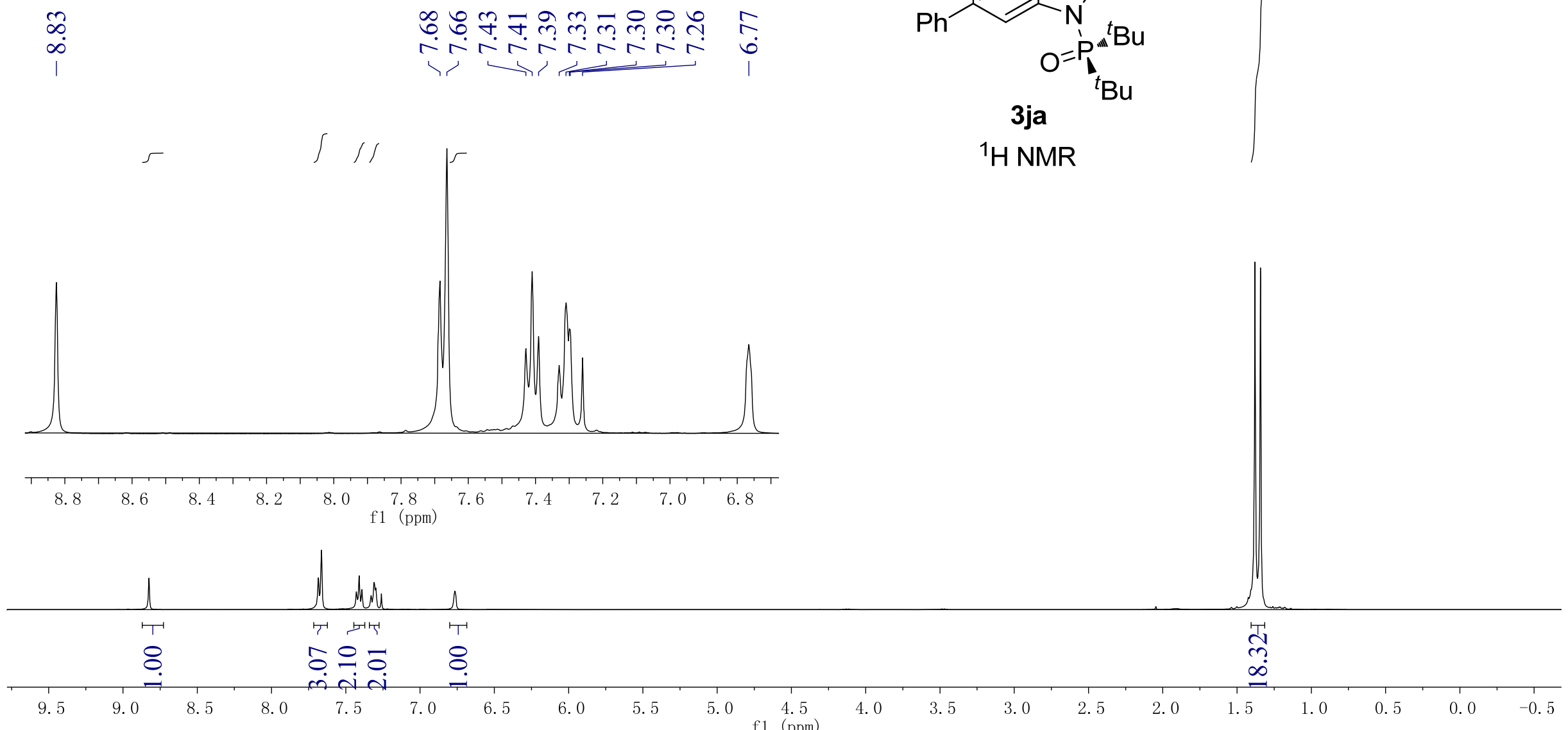

क् से

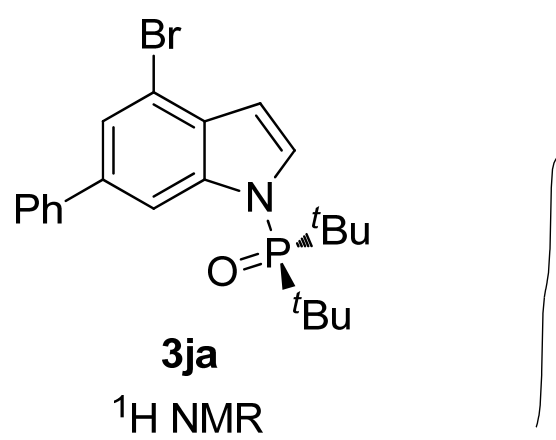




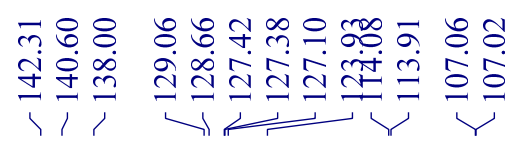

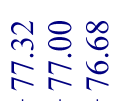

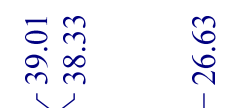

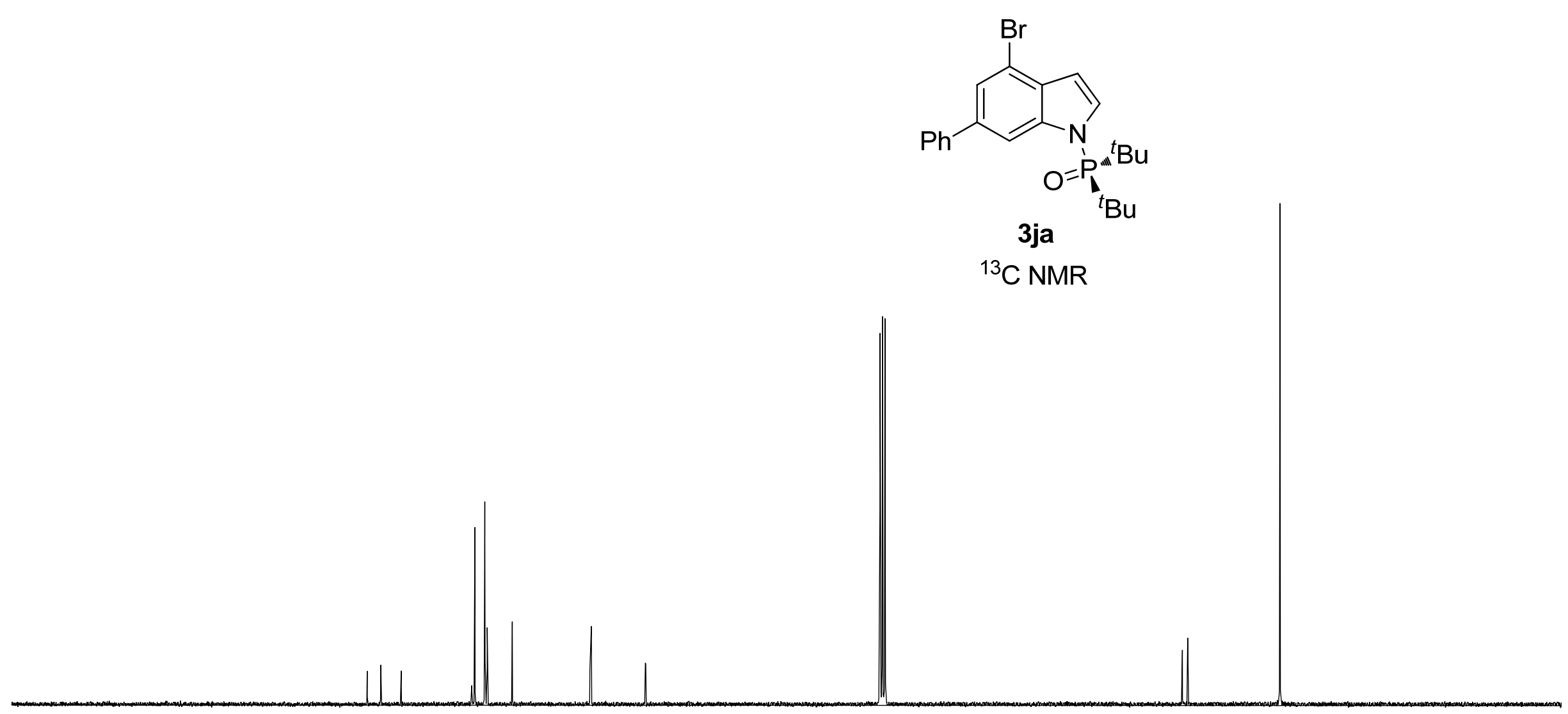

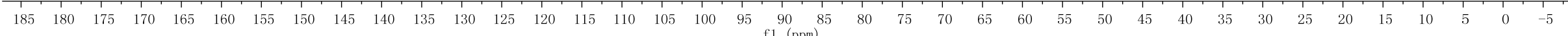


$\stackrel{+}{\dot{f}}$

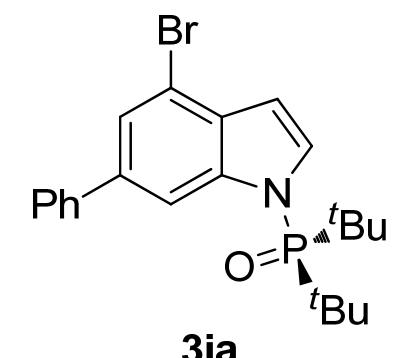

3ja

${ }^{31} \mathrm{P}$ NMR

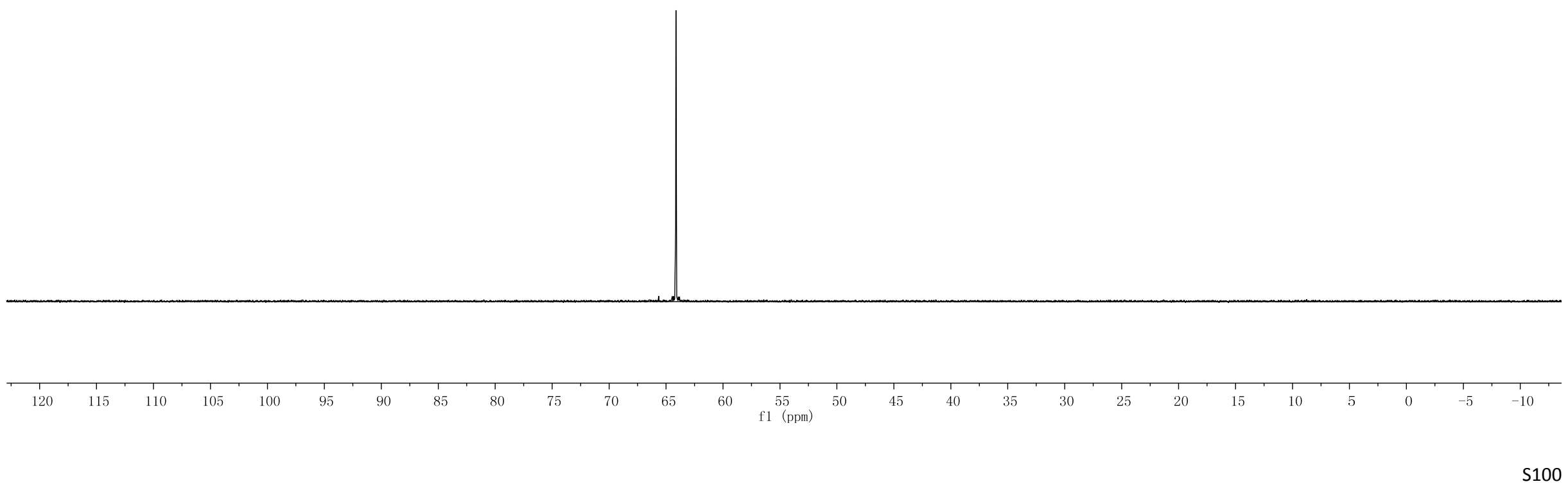




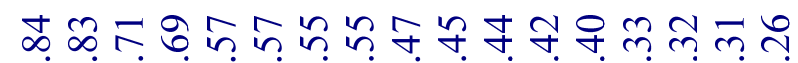

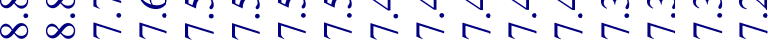

$4+$

Ð $\infty$

$\infty \infty$

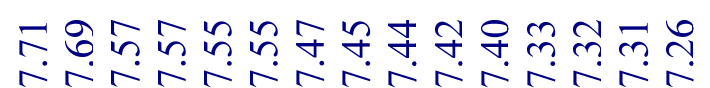

1,1

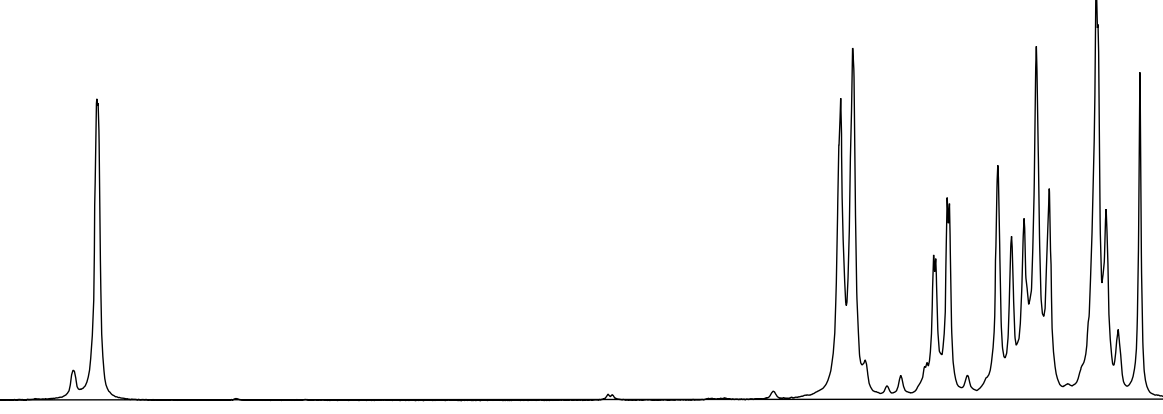

8.9

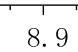

8.7

8.5

8.3

8. 1 (

7.9

7. 7

3

\section{lunth}

Tा 8. i.,-inid 10.5

$\begin{array}{lllllllllll}9.5 & 9.0 & 8.5 & 8.0 & 7.5 & 7.0 & 6.5 & 6.0 & 5.5 & 5.0 & 4.5\end{array}$

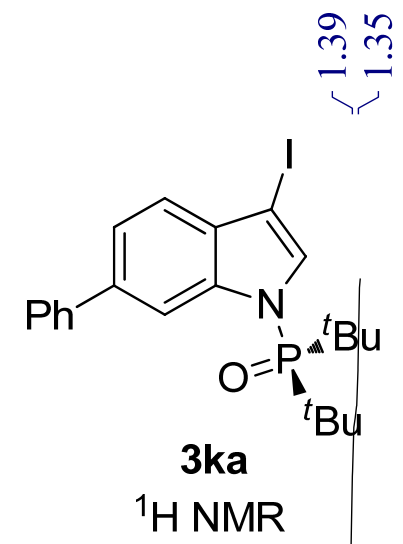

H NMR

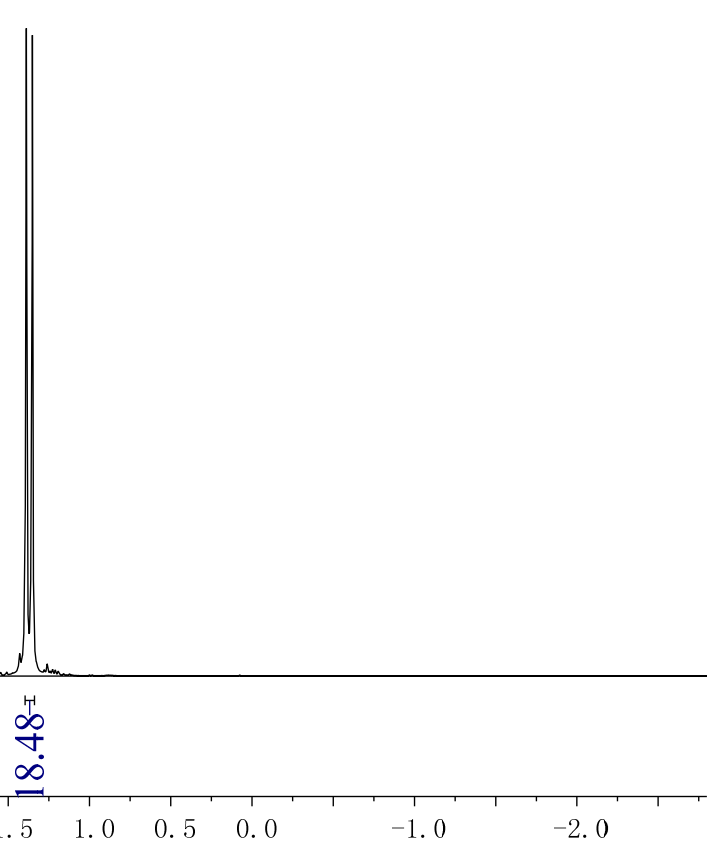



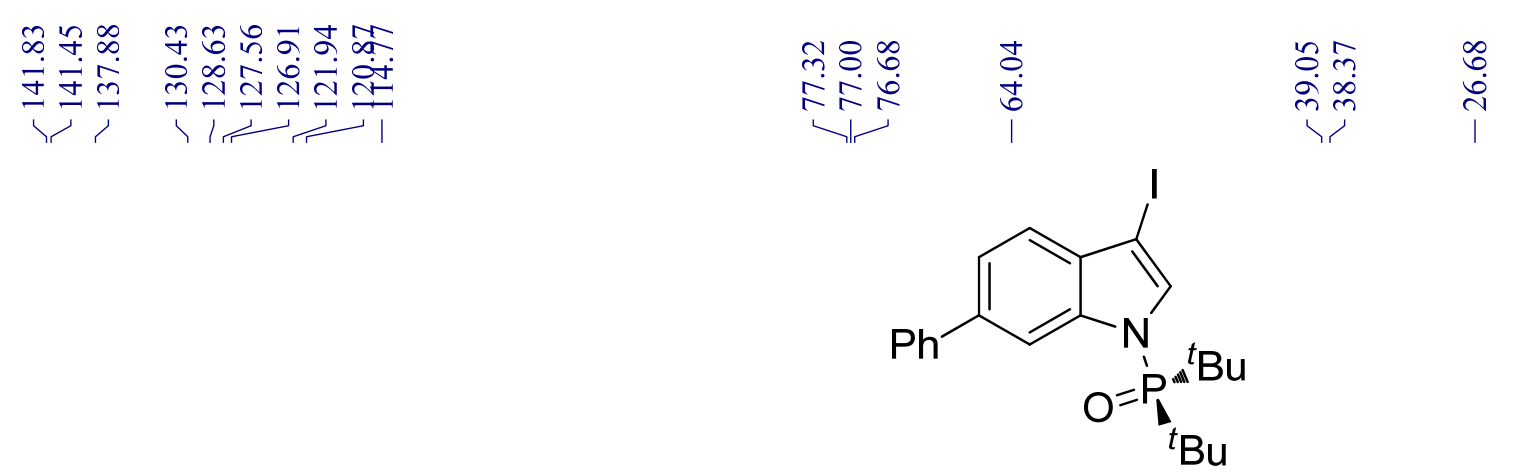

3ka

${ }^{13} \mathrm{C}$ NMR

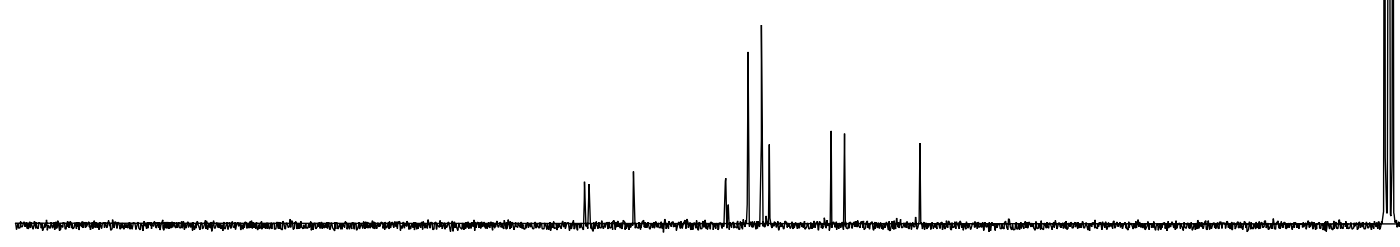


ํํㅁ
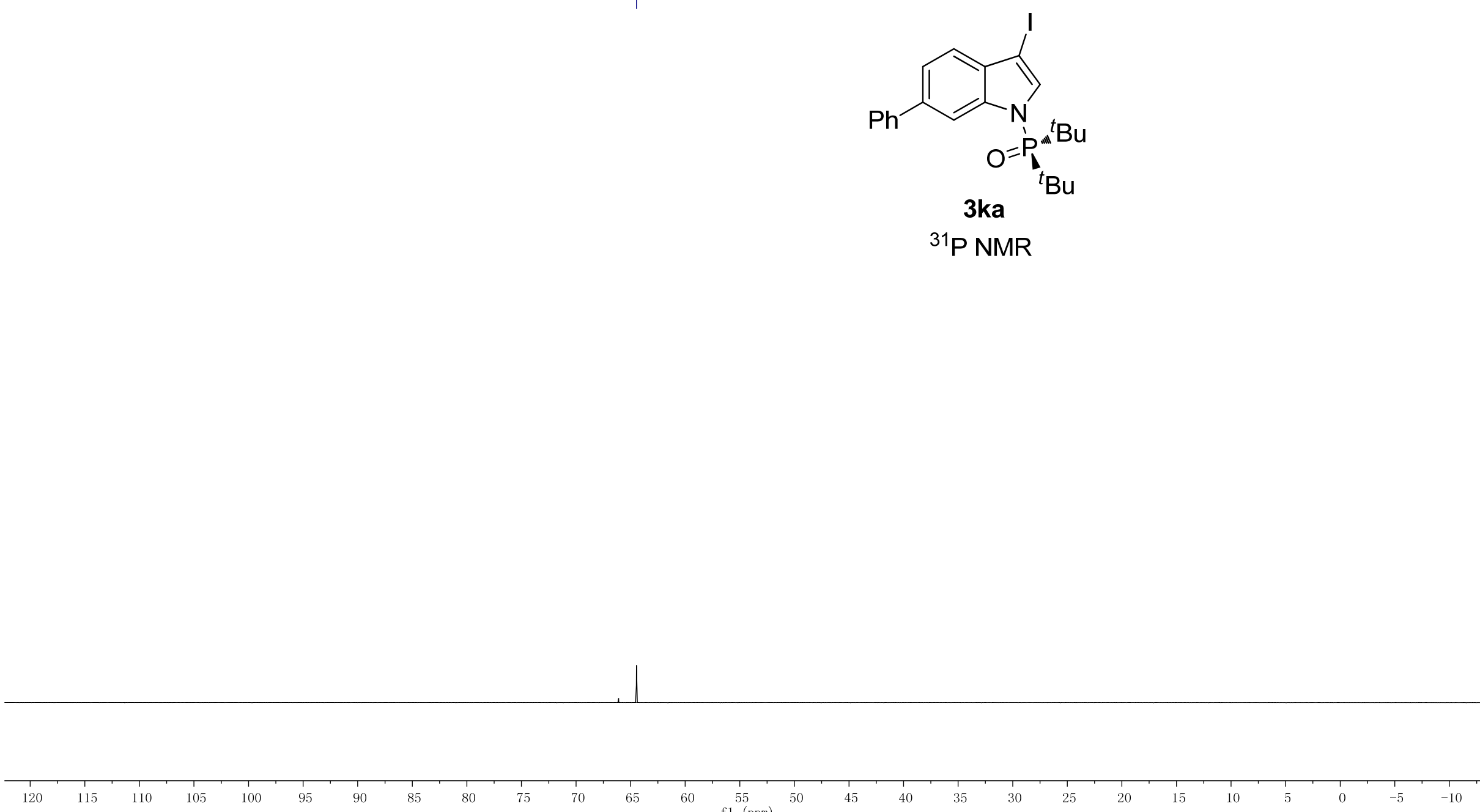


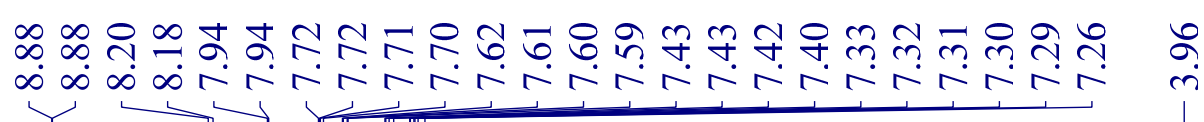

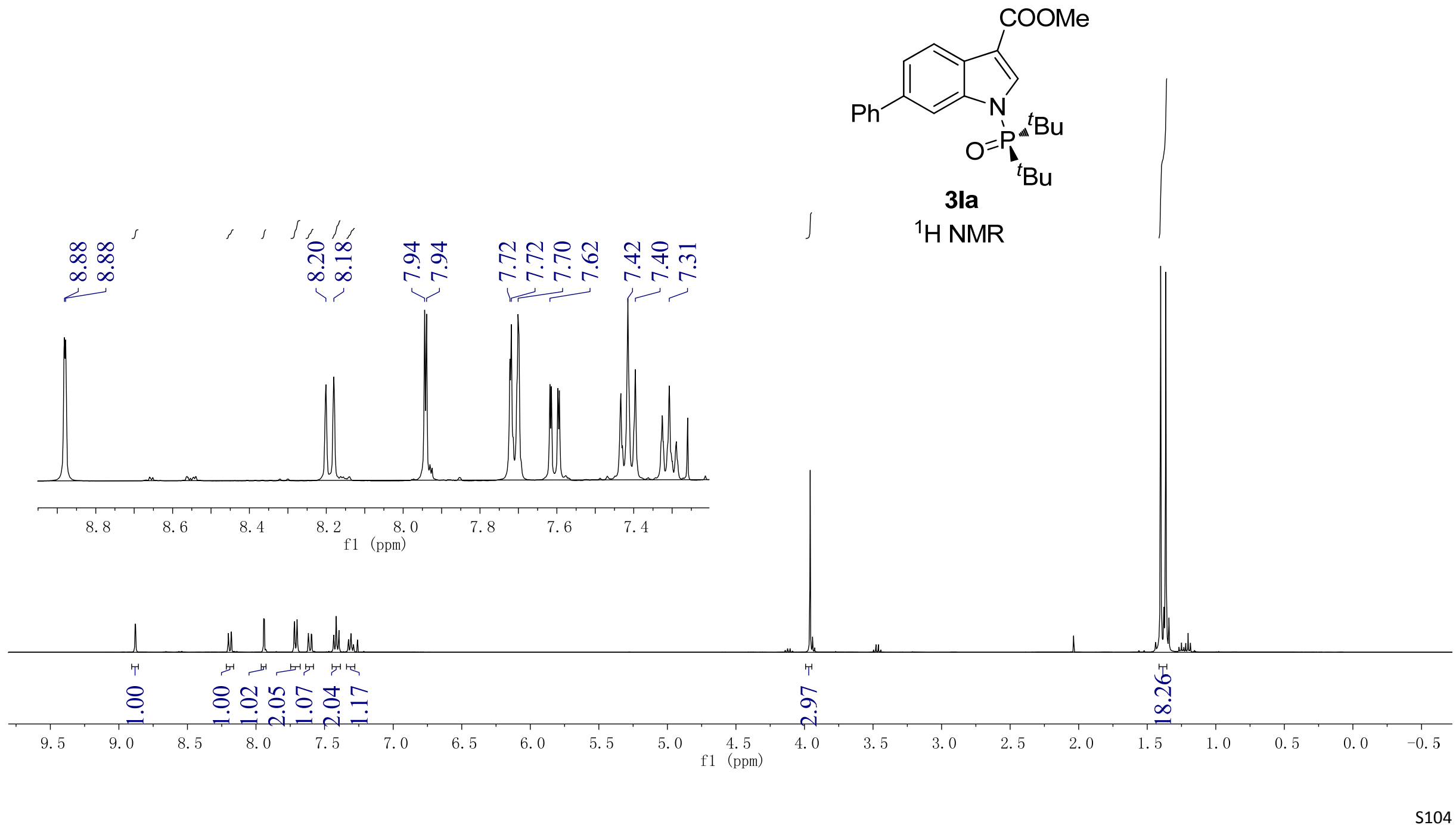




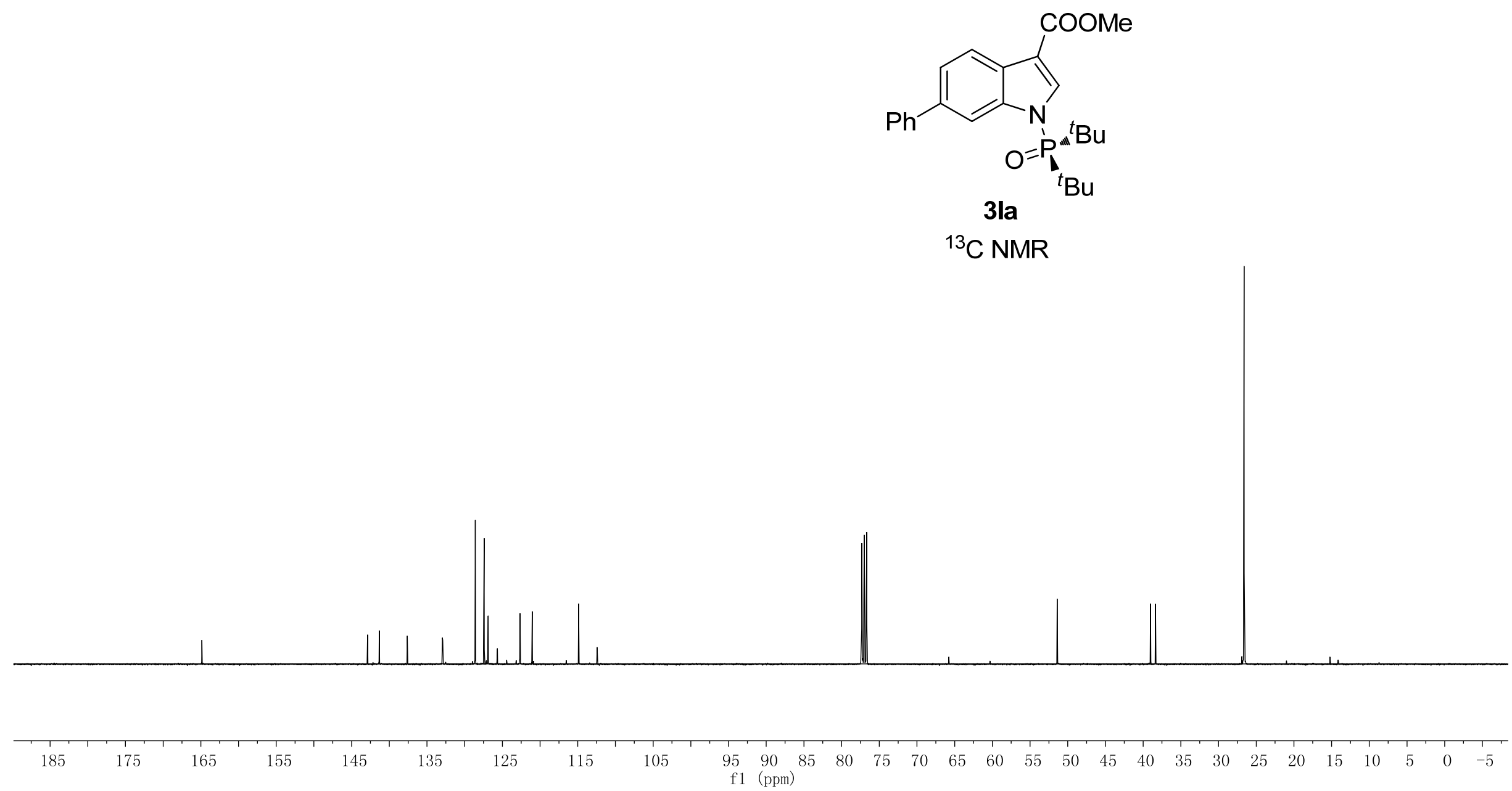




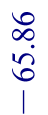

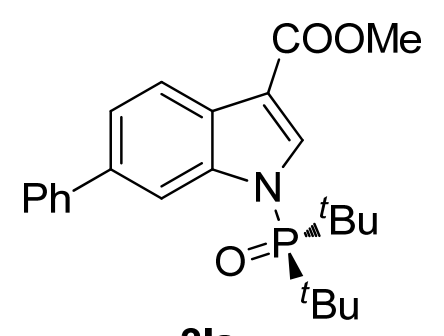

3la

${ }^{31} \mathrm{P}$ NMR

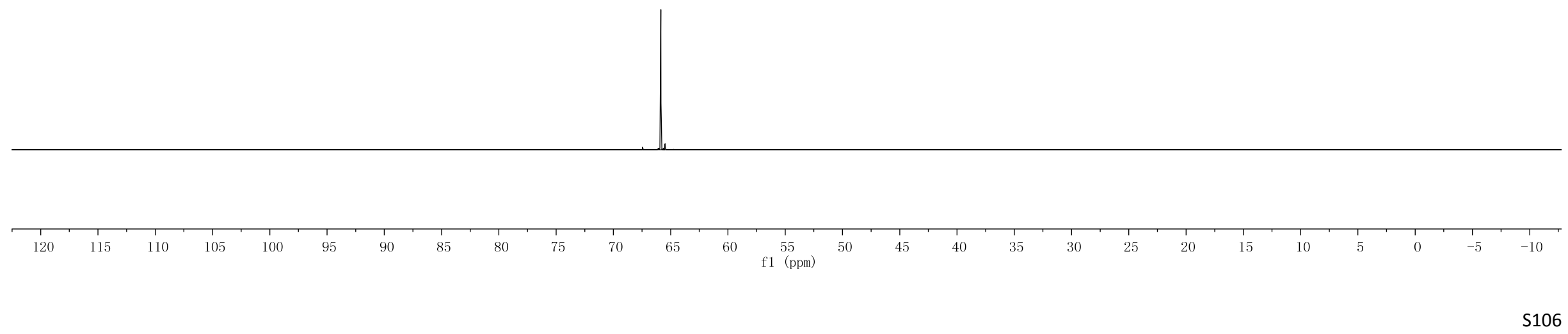




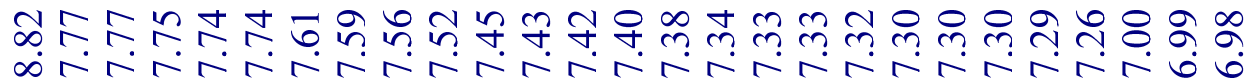

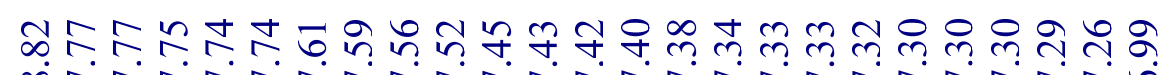

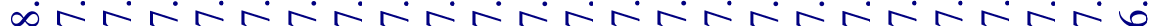
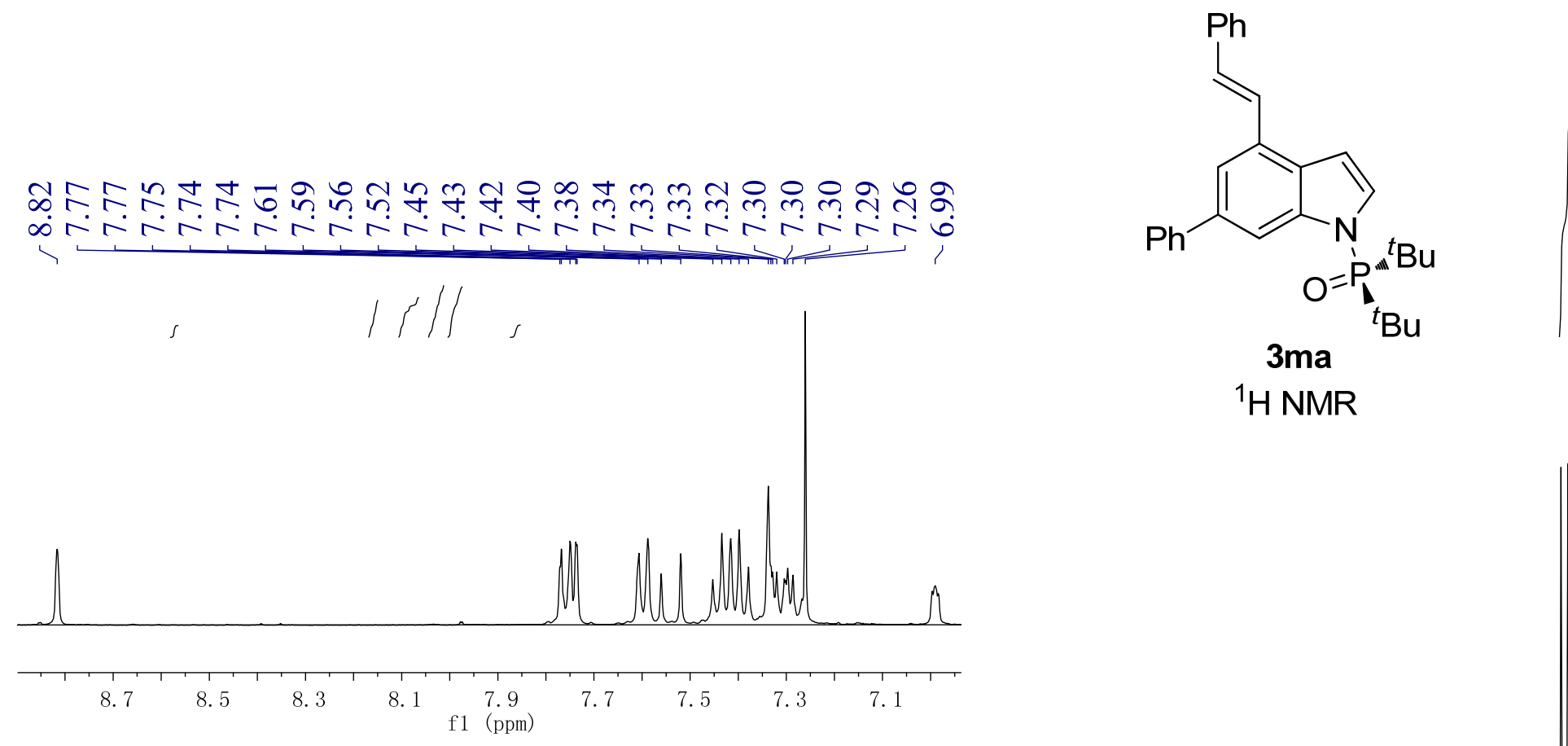

3ma

${ }^{1} \mathrm{H}$ NMR

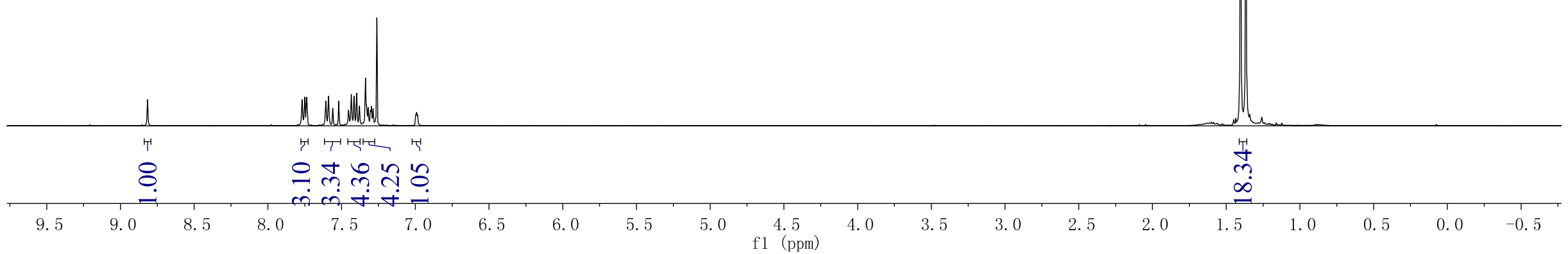




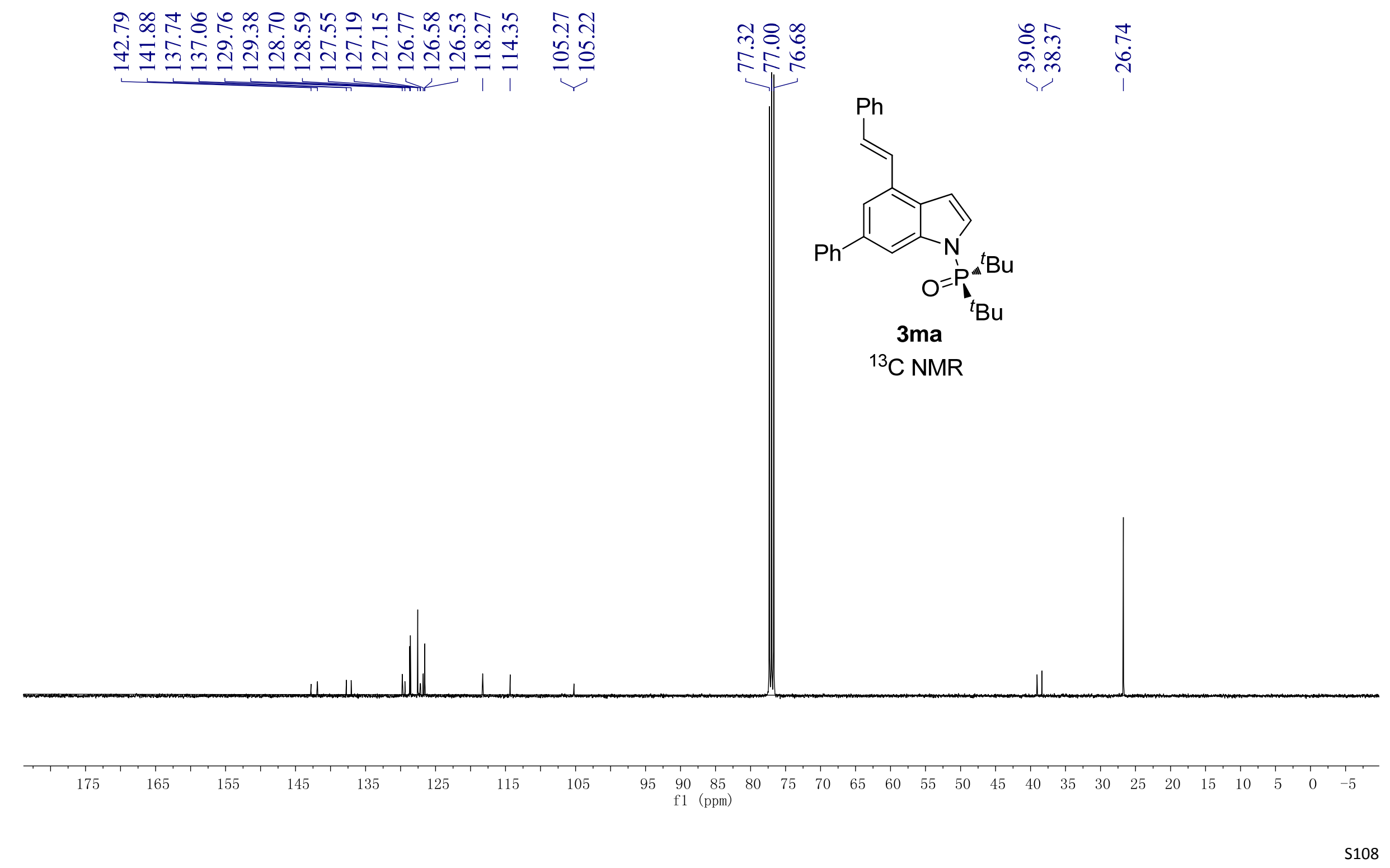




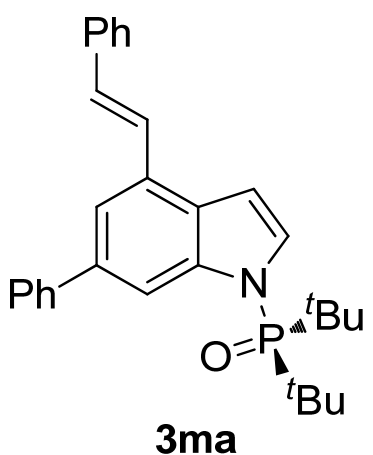

${ }^{31} \mathrm{P}$ NMR

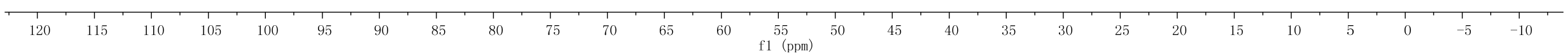




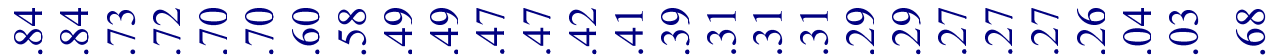

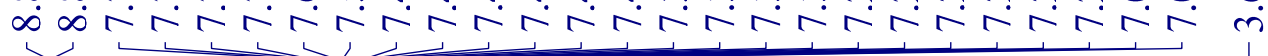

চ

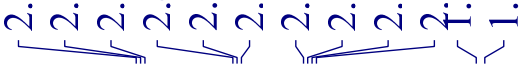

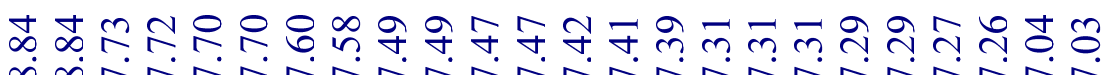
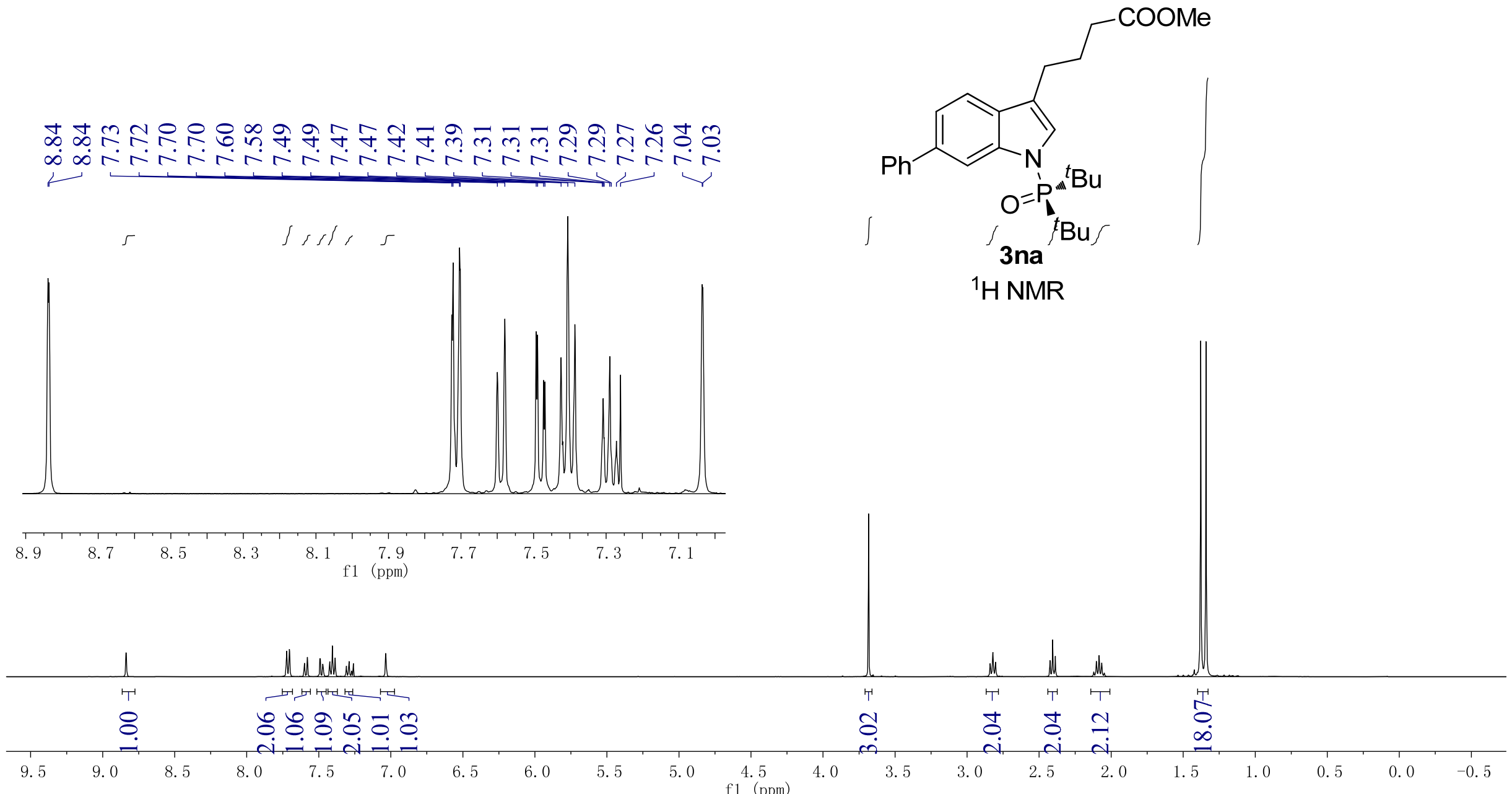


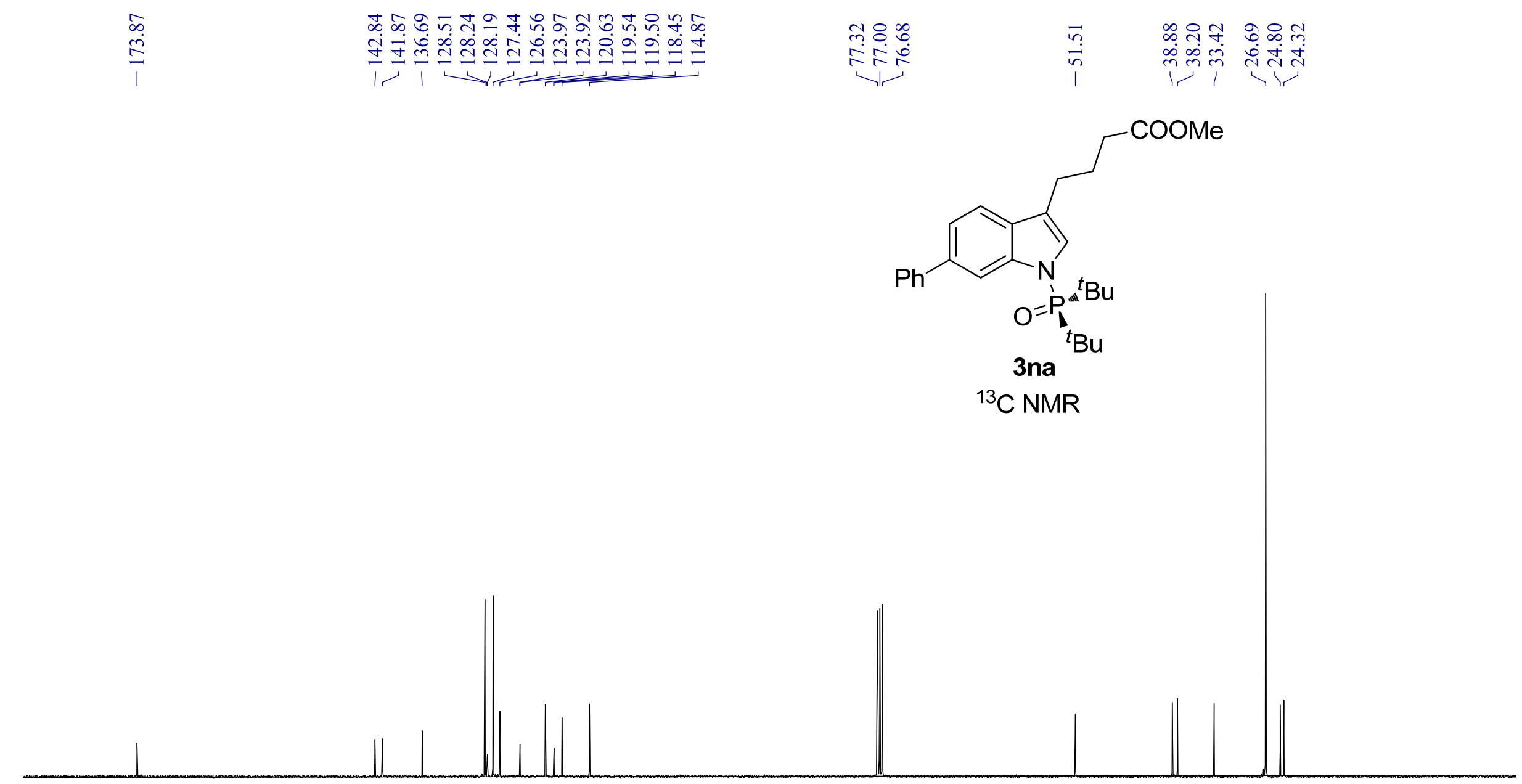

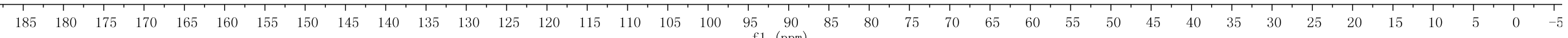


0
0
0
$i$

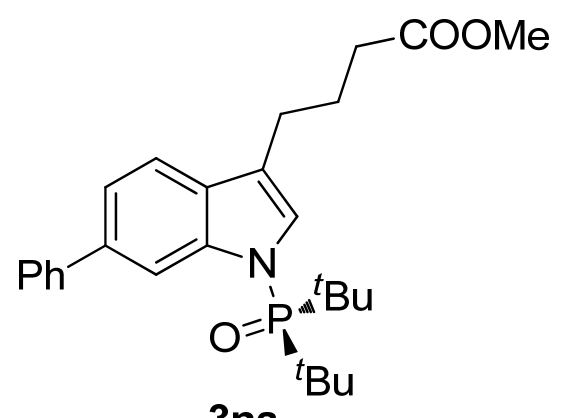

${ }^{31} \mathrm{P}$ NMR

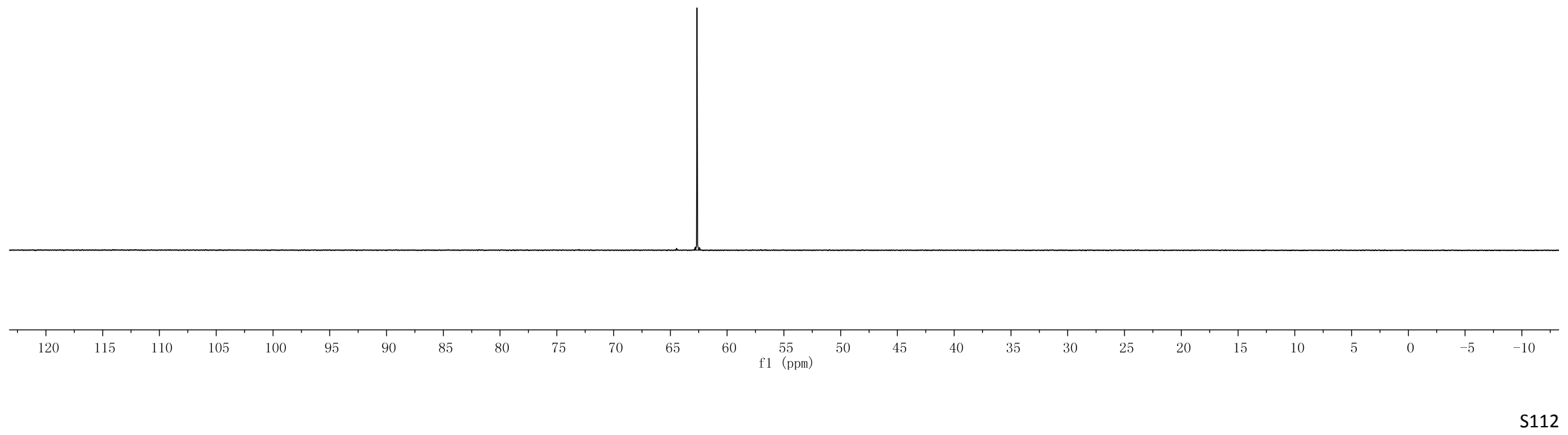




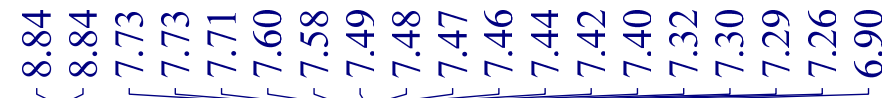

ナ

$\infty$
$\infty$
$\infty$

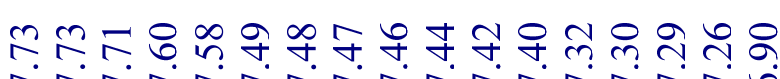

$<$
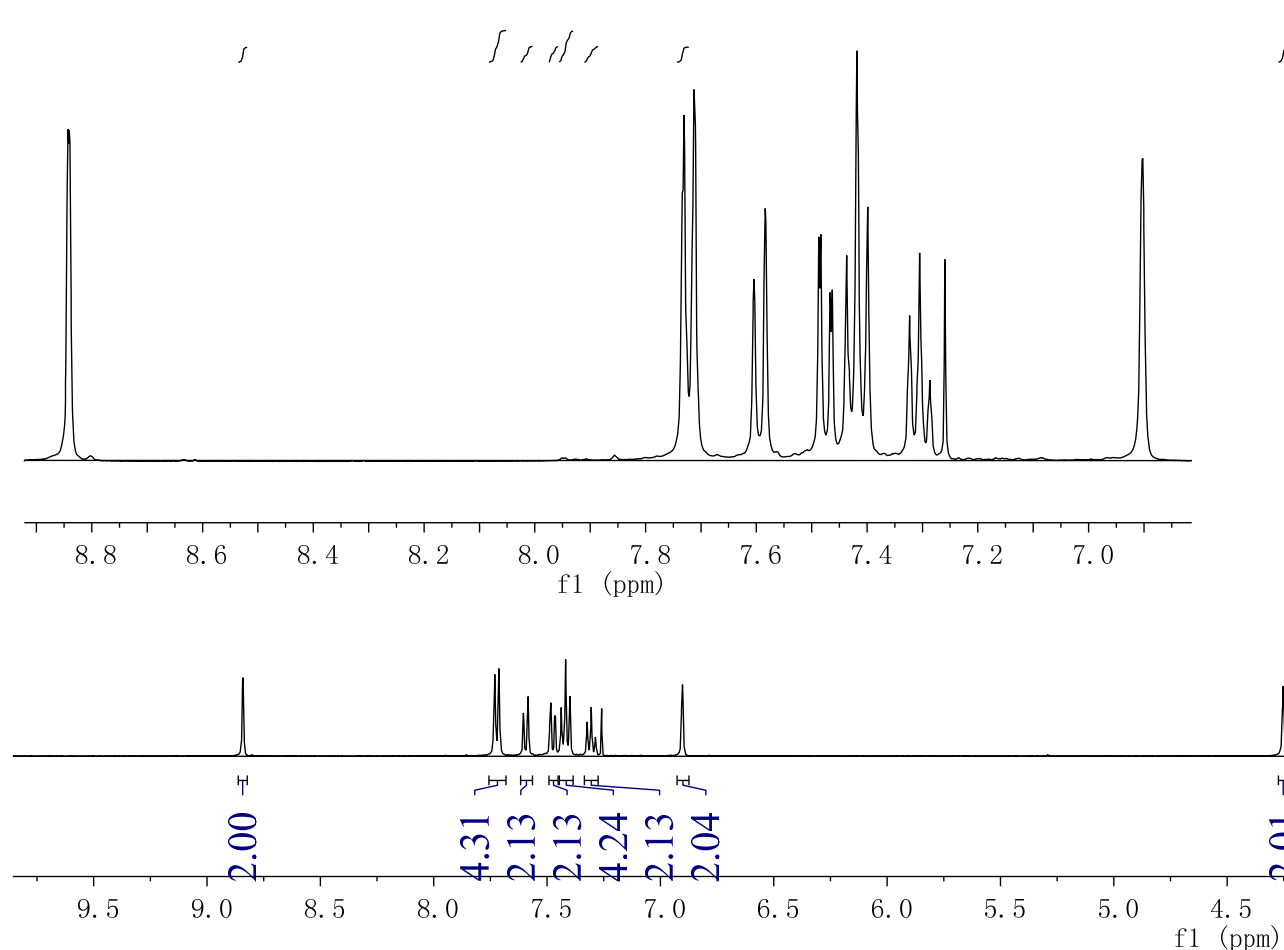

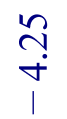
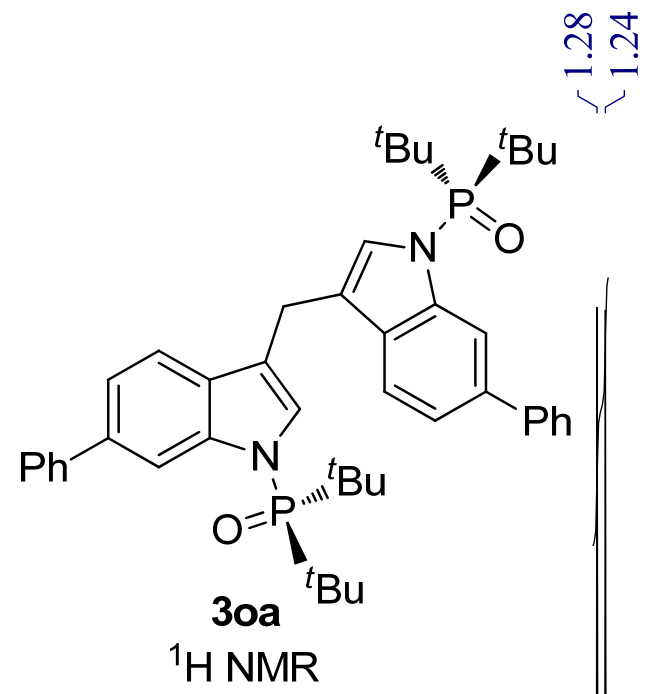


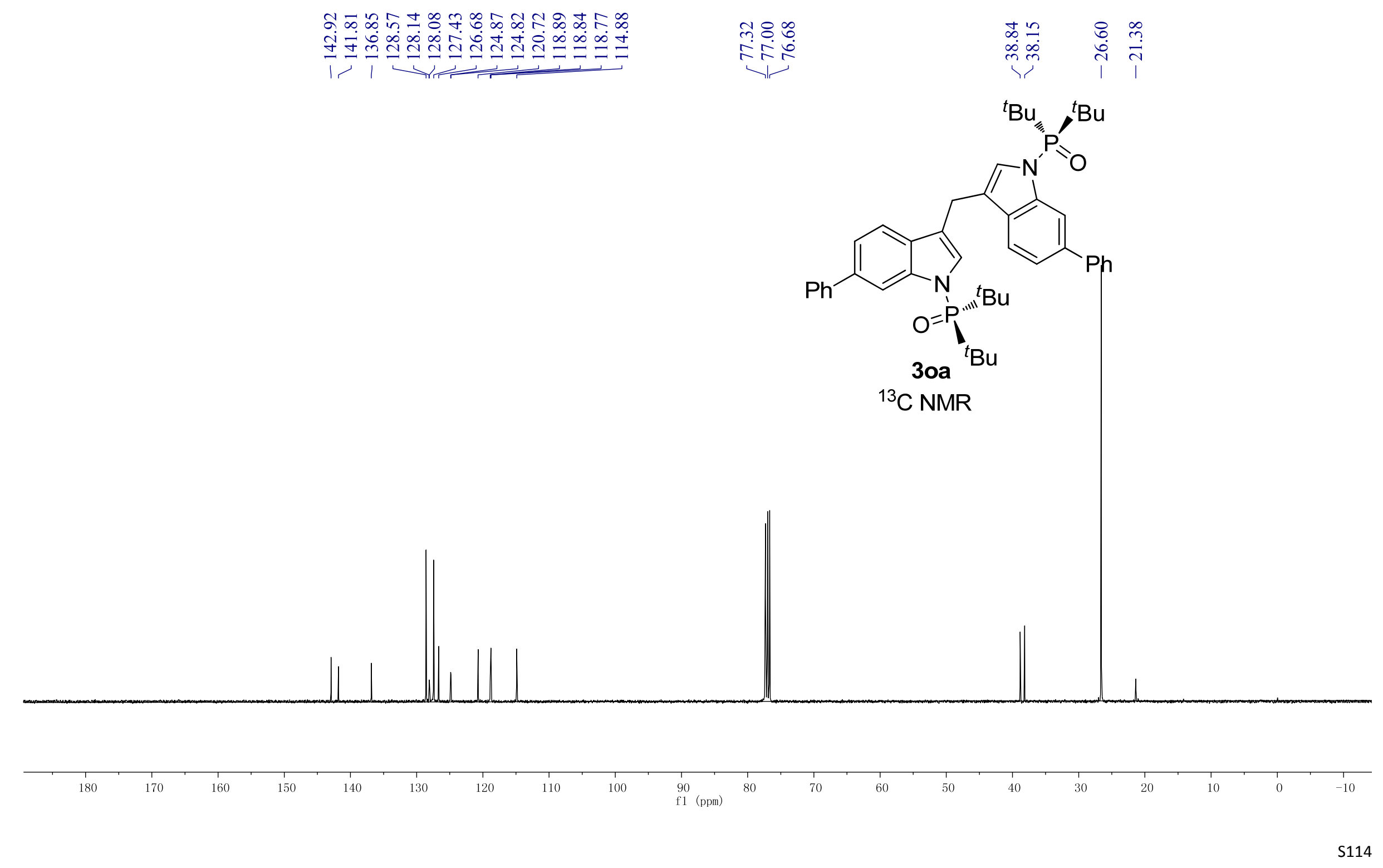


$\stackrel{0}{\mathrm{i}}$
i
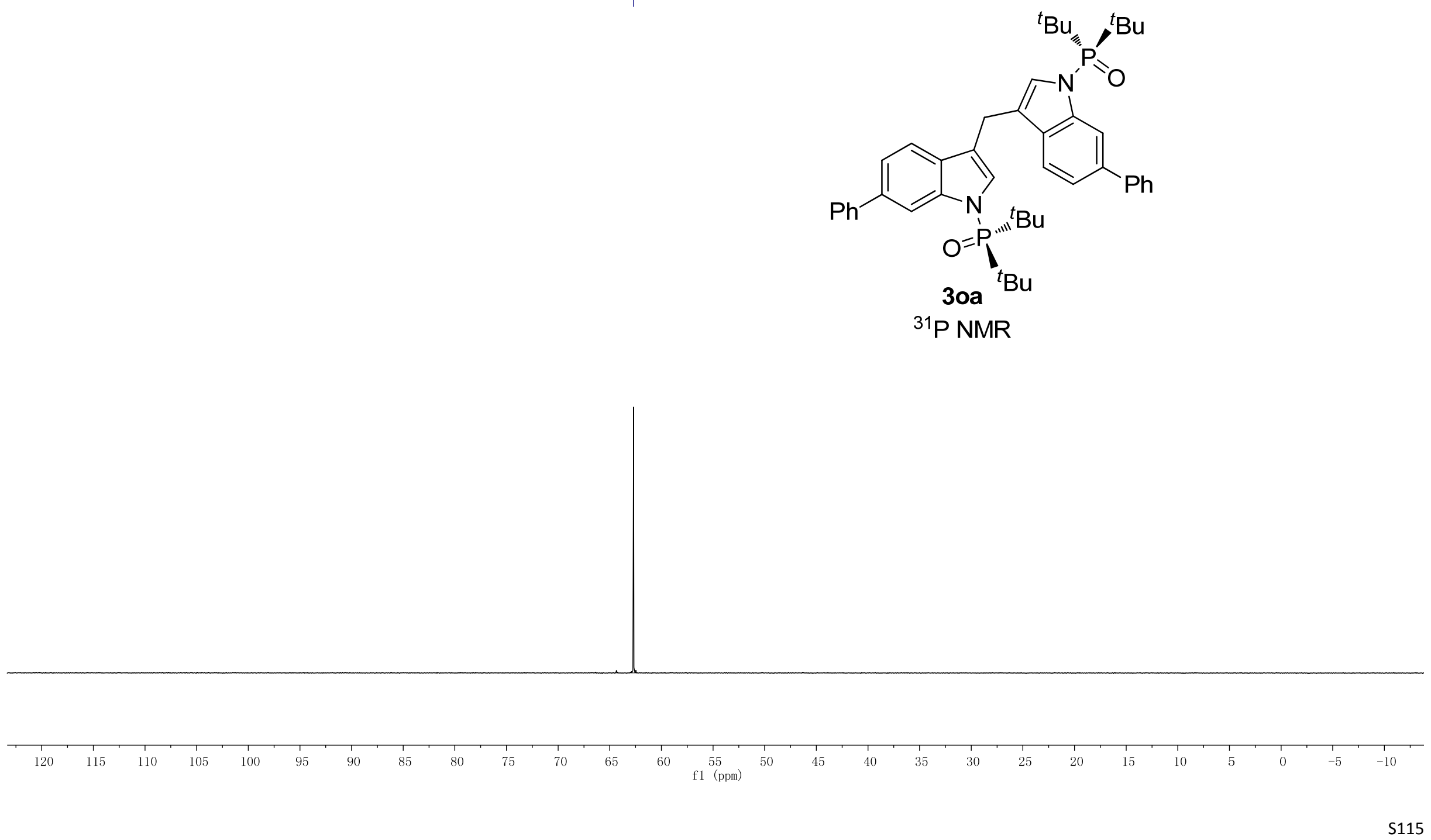


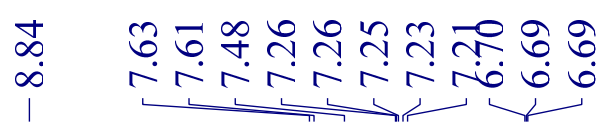
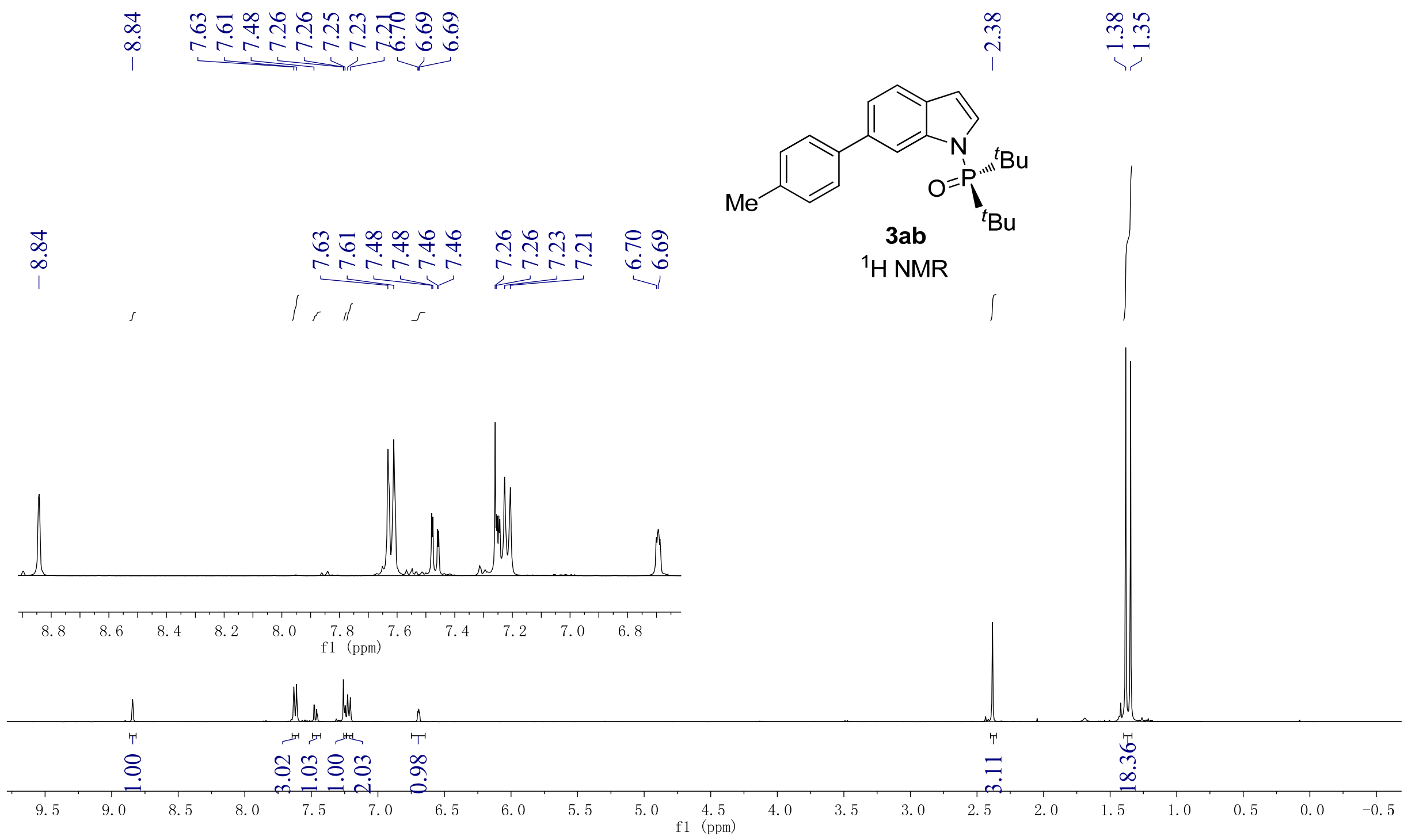


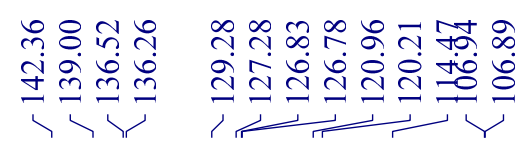

$$
\begin{aligned}
& \text { กิ } \\
& \text { กำ }
\end{aligned}
$$

$$
\text { a. ते }
$$

in

$\underset{\substack{1\\}}{\stackrel{4}{+}}$

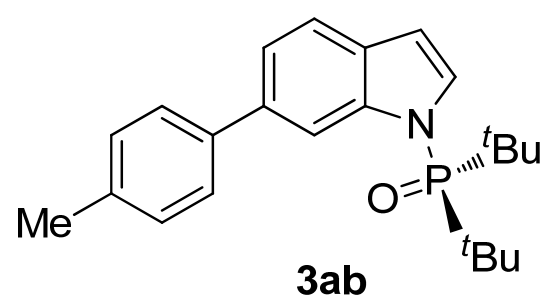

${ }^{13} \mathrm{C}$ NMR

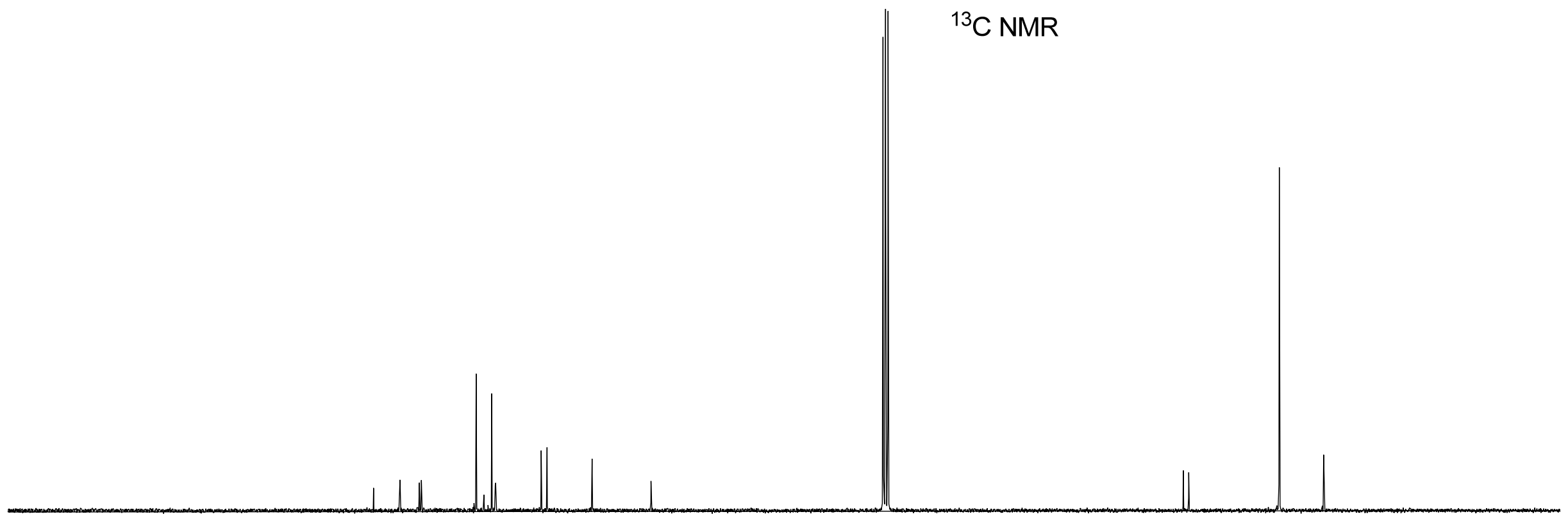

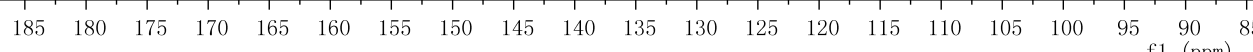


ลे

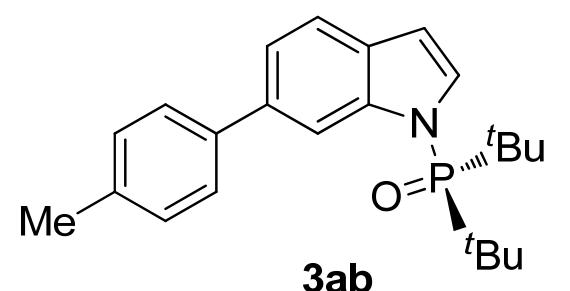

${ }^{31} \mathrm{P}$ NMR

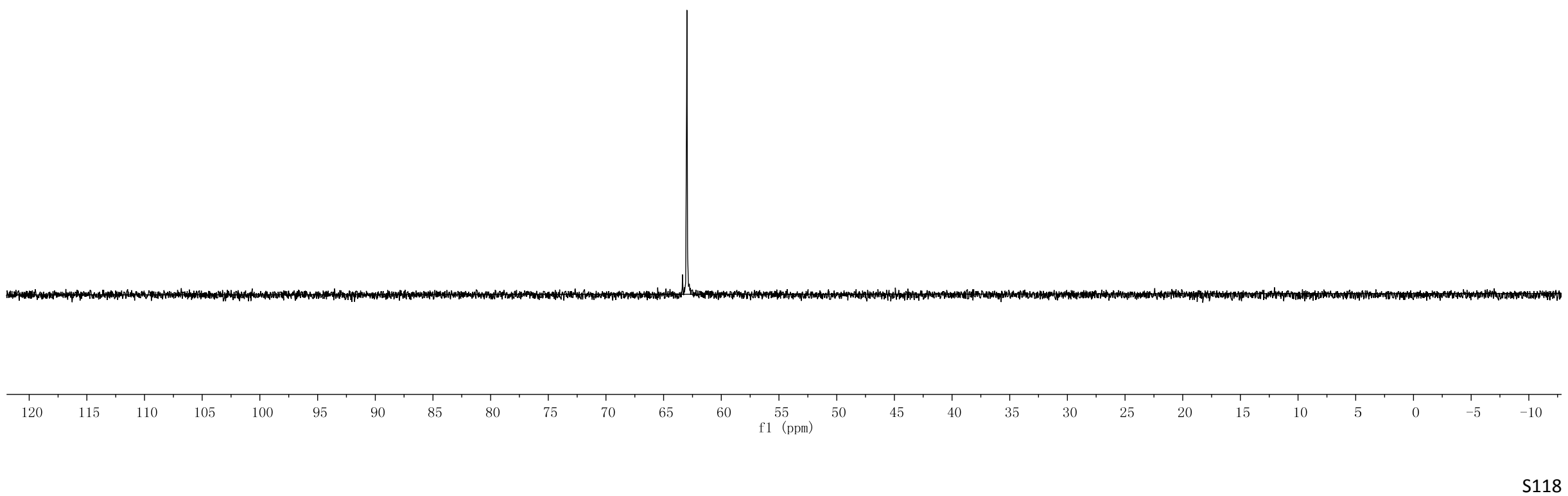


ฌ

क N N N N N No

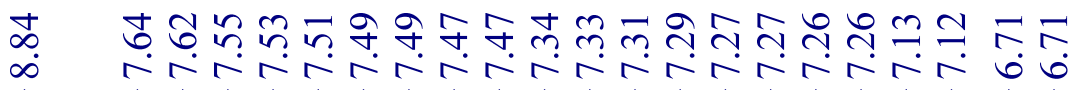

L्य

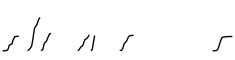

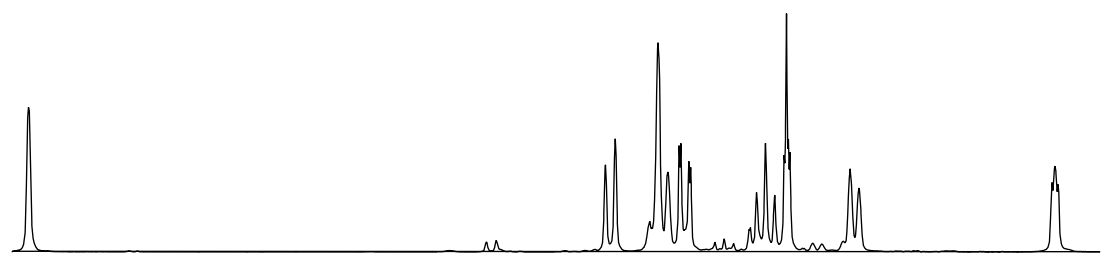

$\begin{array}{lllllllllll}8.8 & 8.6 & 8.4 & 8.2 & 8.0 & 7.8 & 7.6 & 7.4 & 7.2 & 7.0 & 6.8\end{array}$

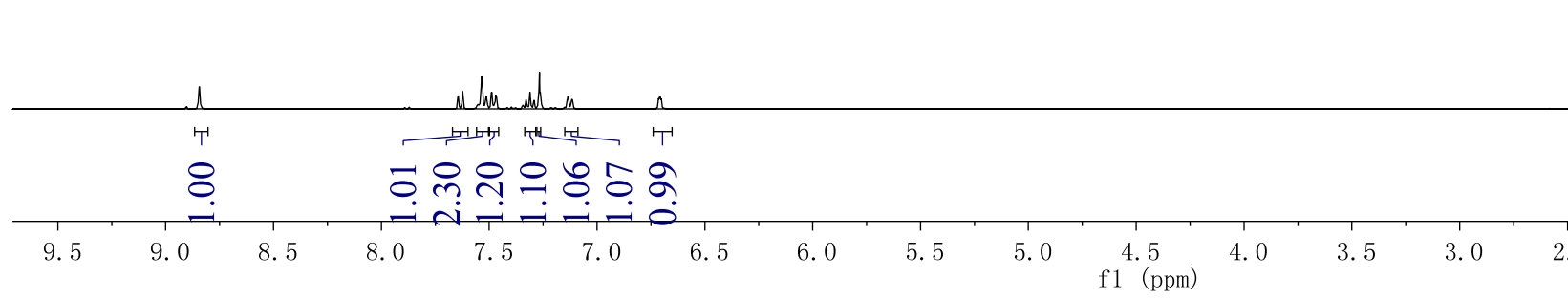

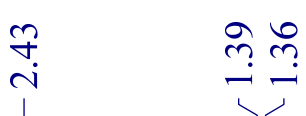

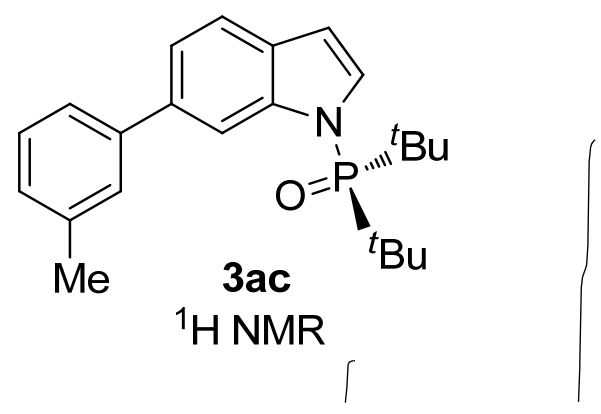



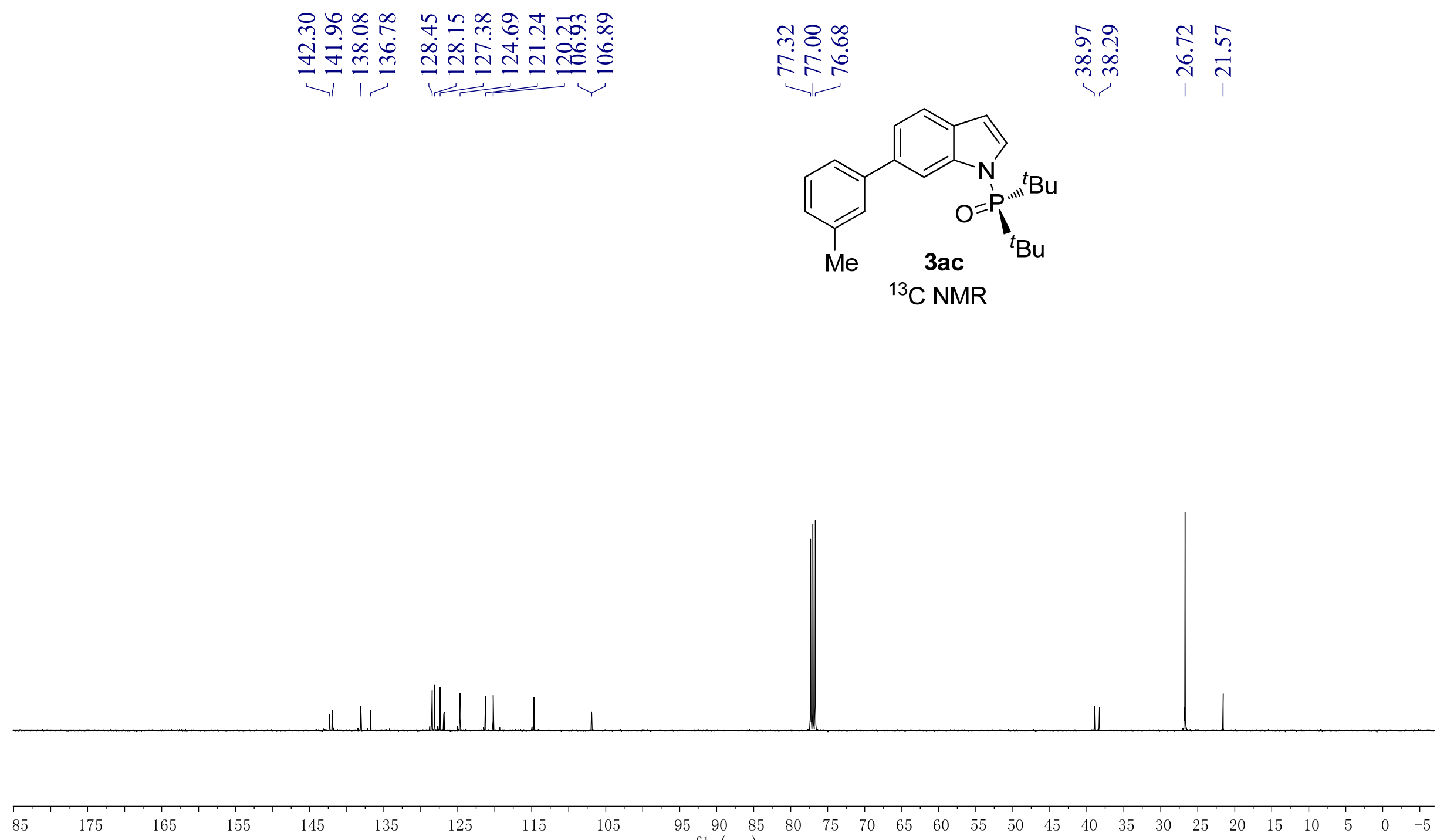

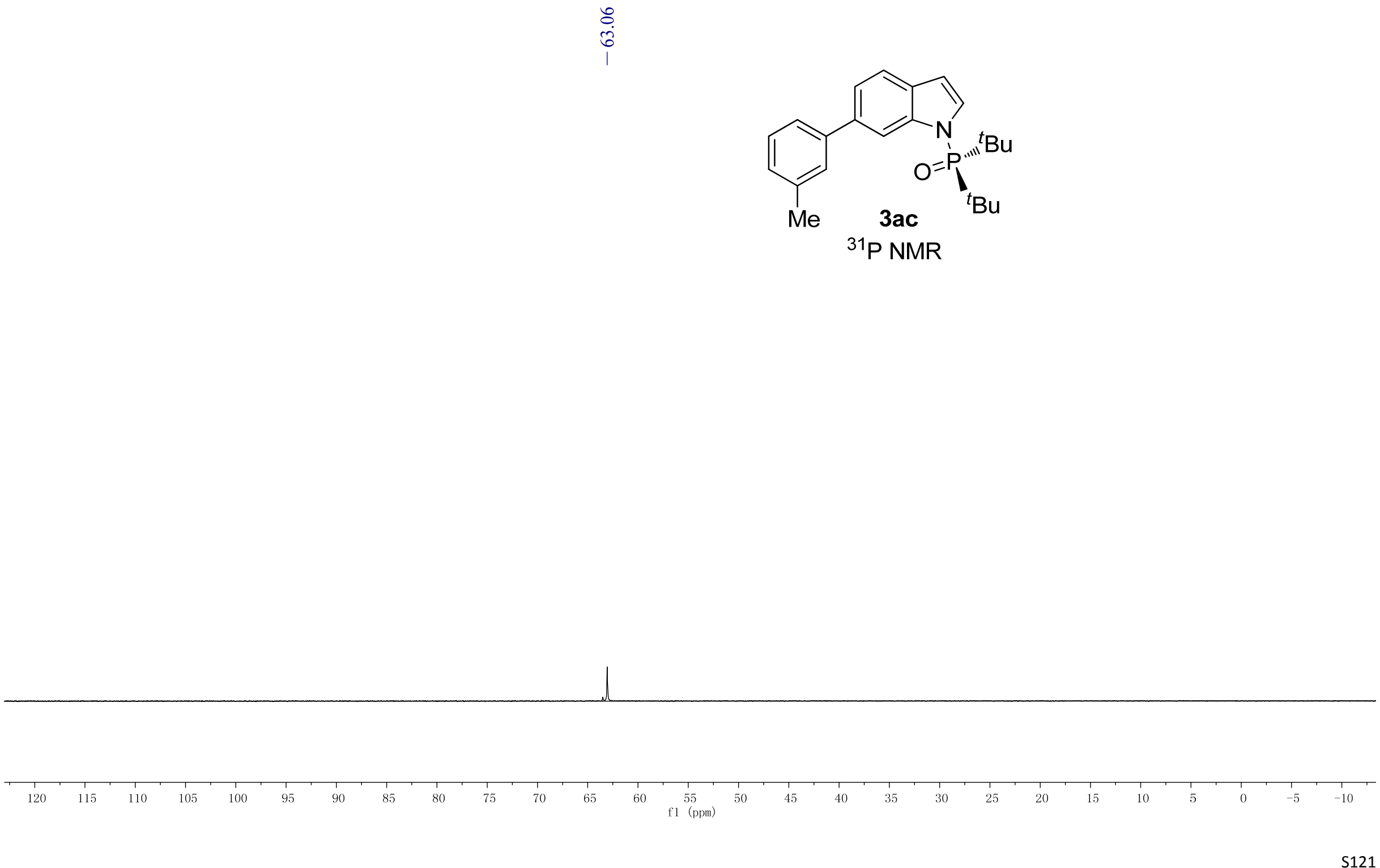

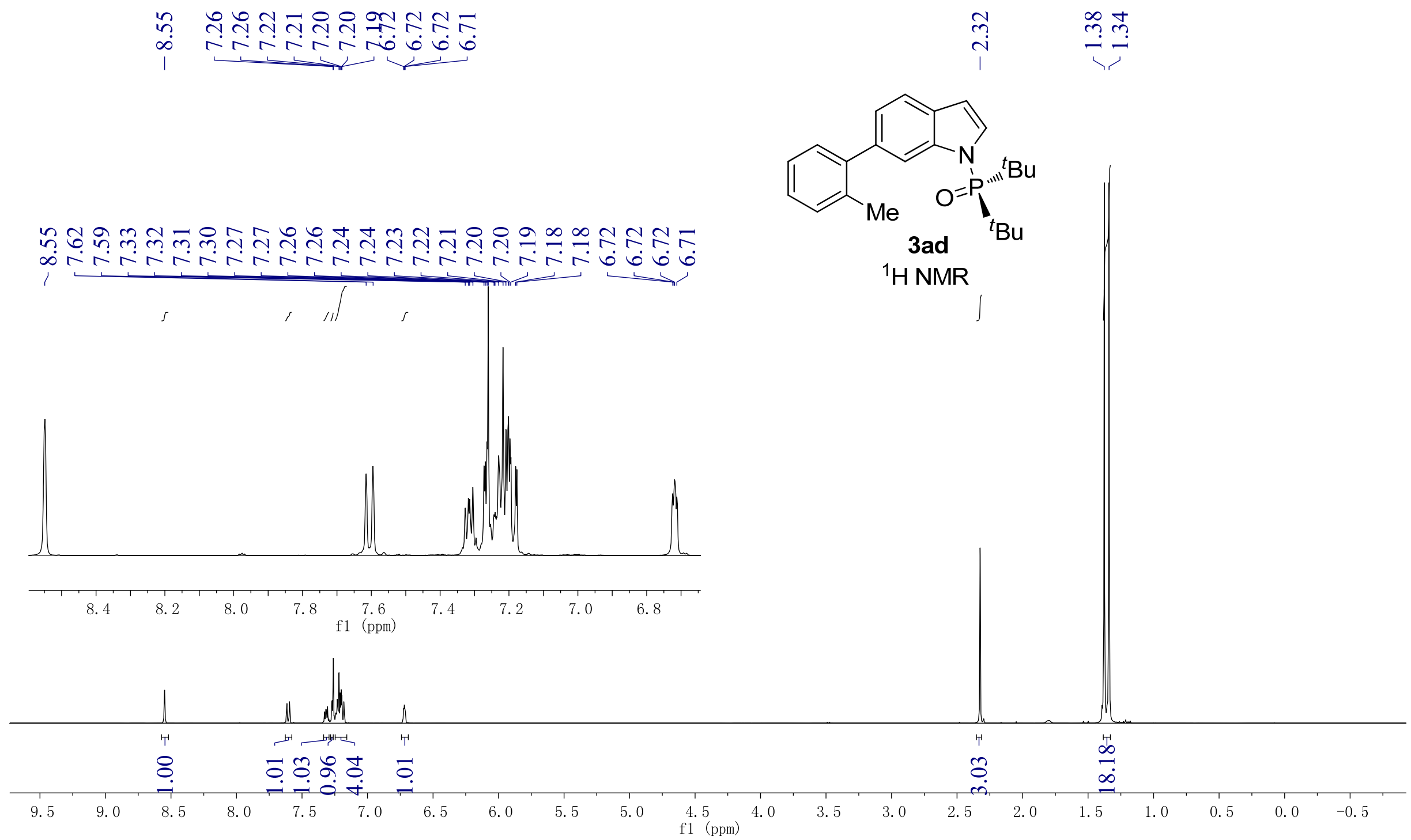


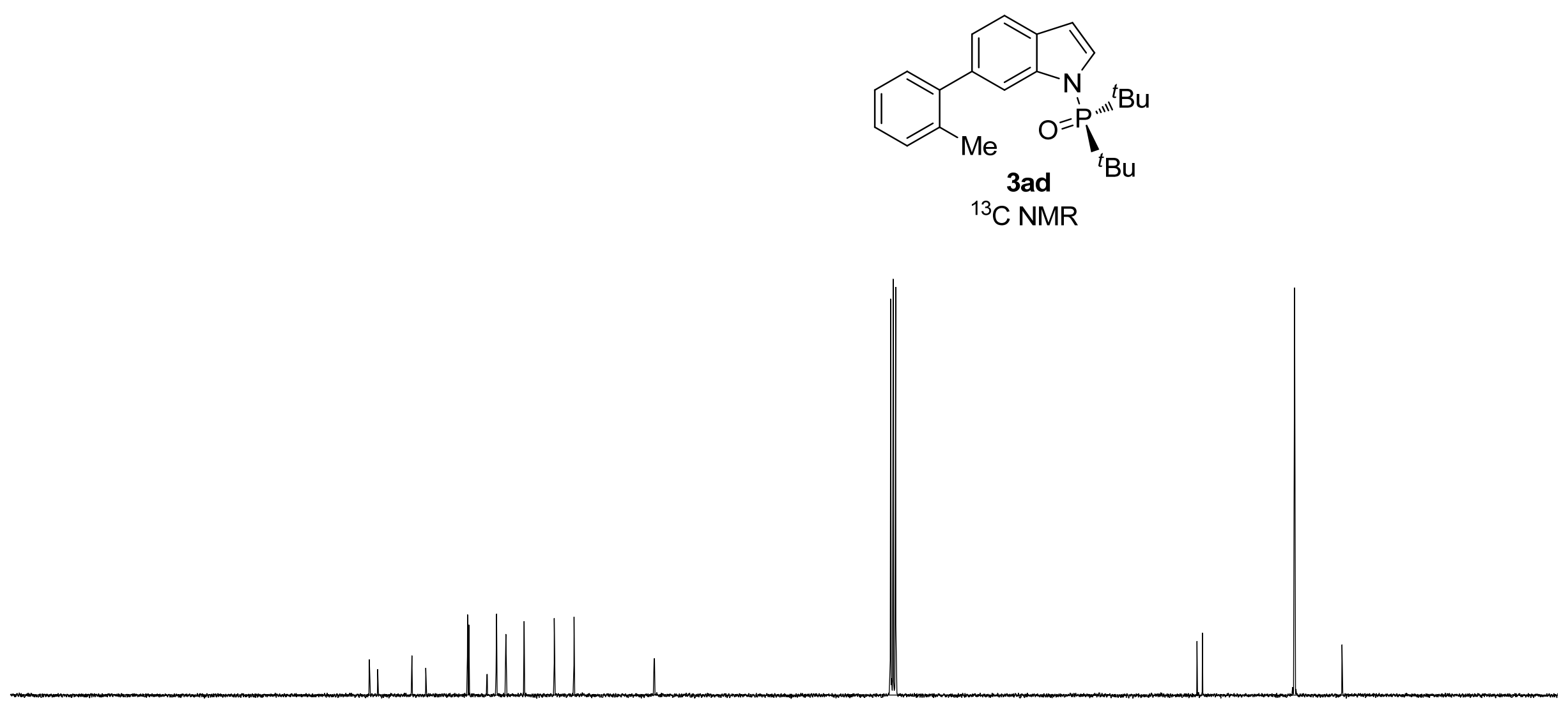

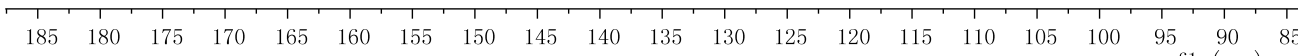


న్
రై
1

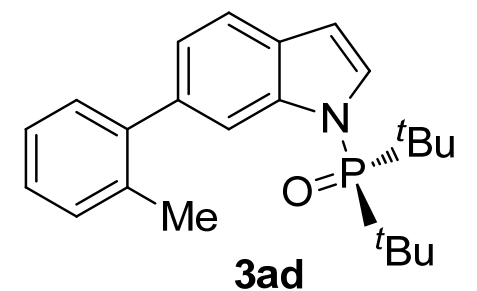

${ }^{31} \mathrm{P}$ NMR

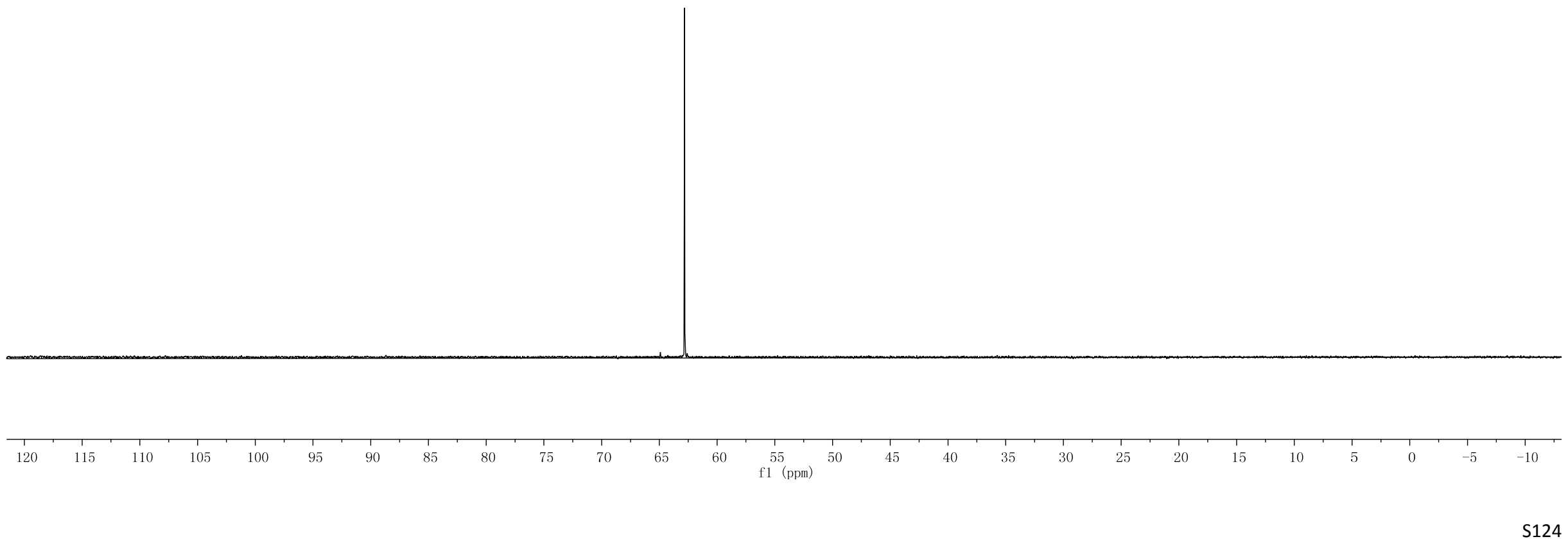




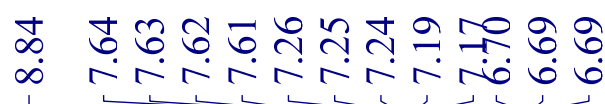

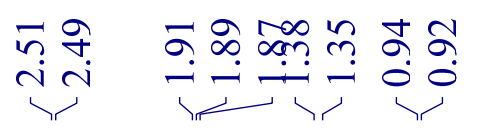

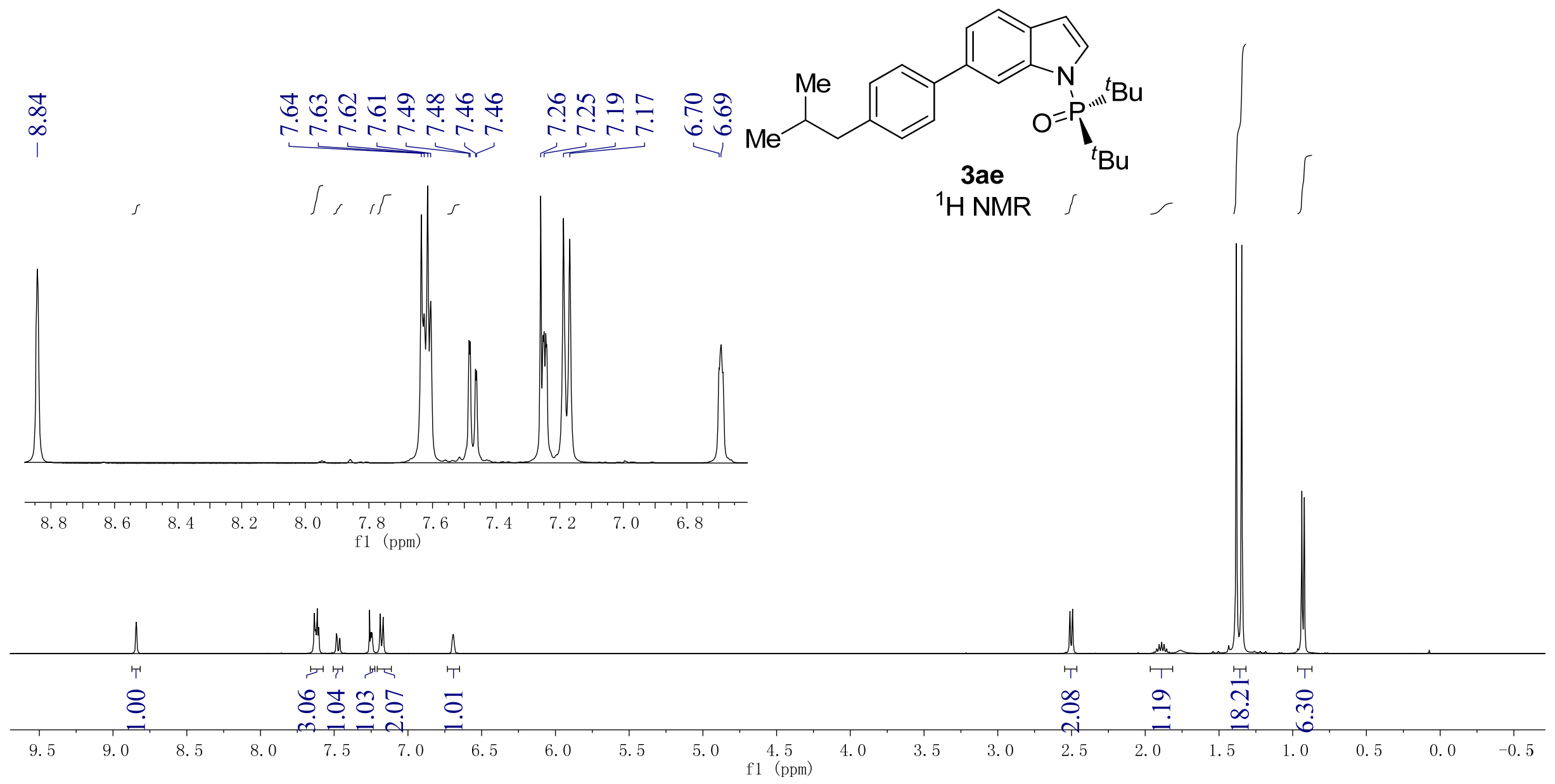




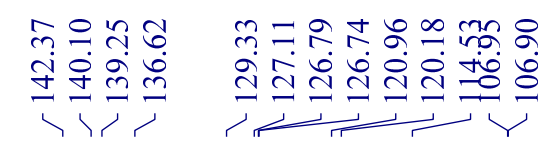

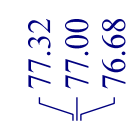

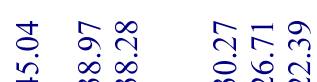

i m $m$ m

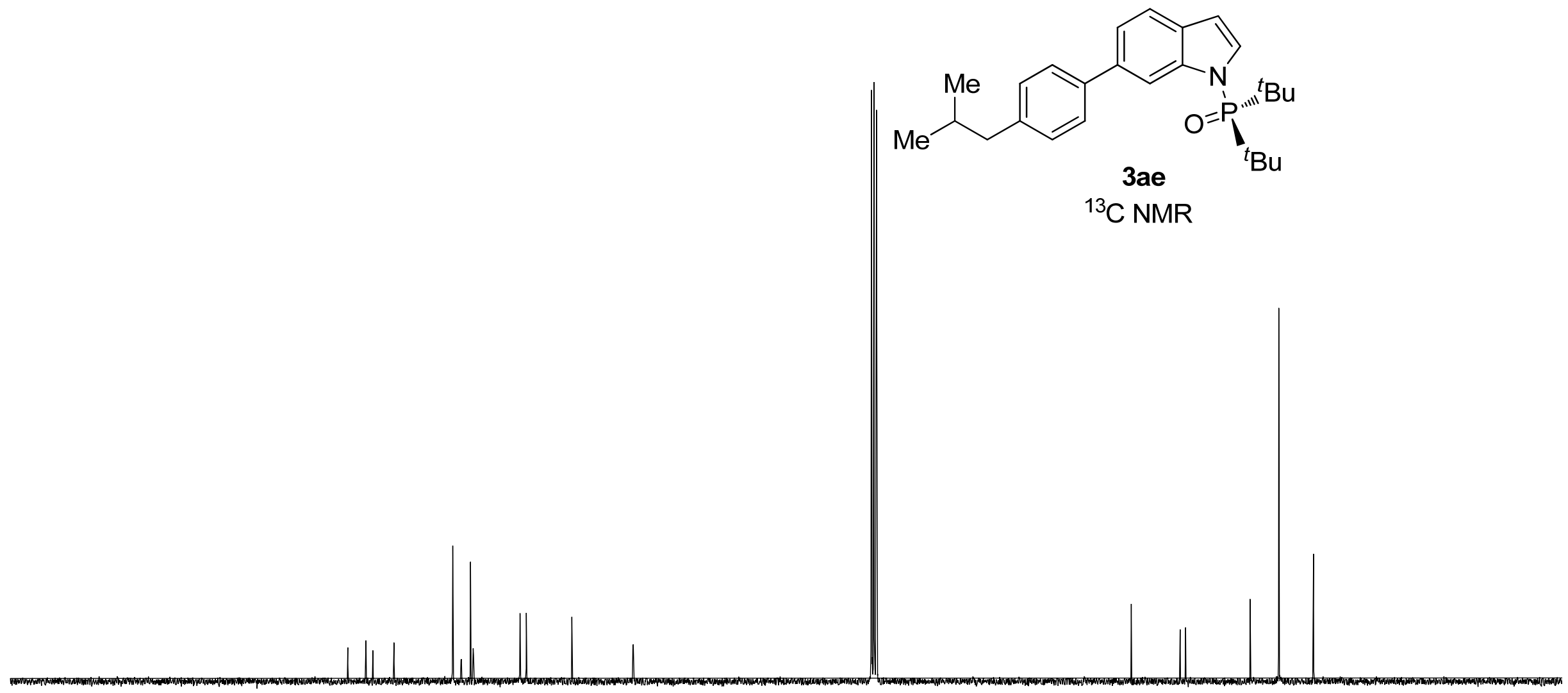

$\begin{array}{lllllllllllllllllllll}180 & 175 & 170 & 165 & 160 & 155 & 150 & 145 & 140 & 135 & 130 & 125 & 120 & 115 & 110 & 105 & 100 & 95 & 90 & 85\end{array}$ 
$\hat{a}$
i
1
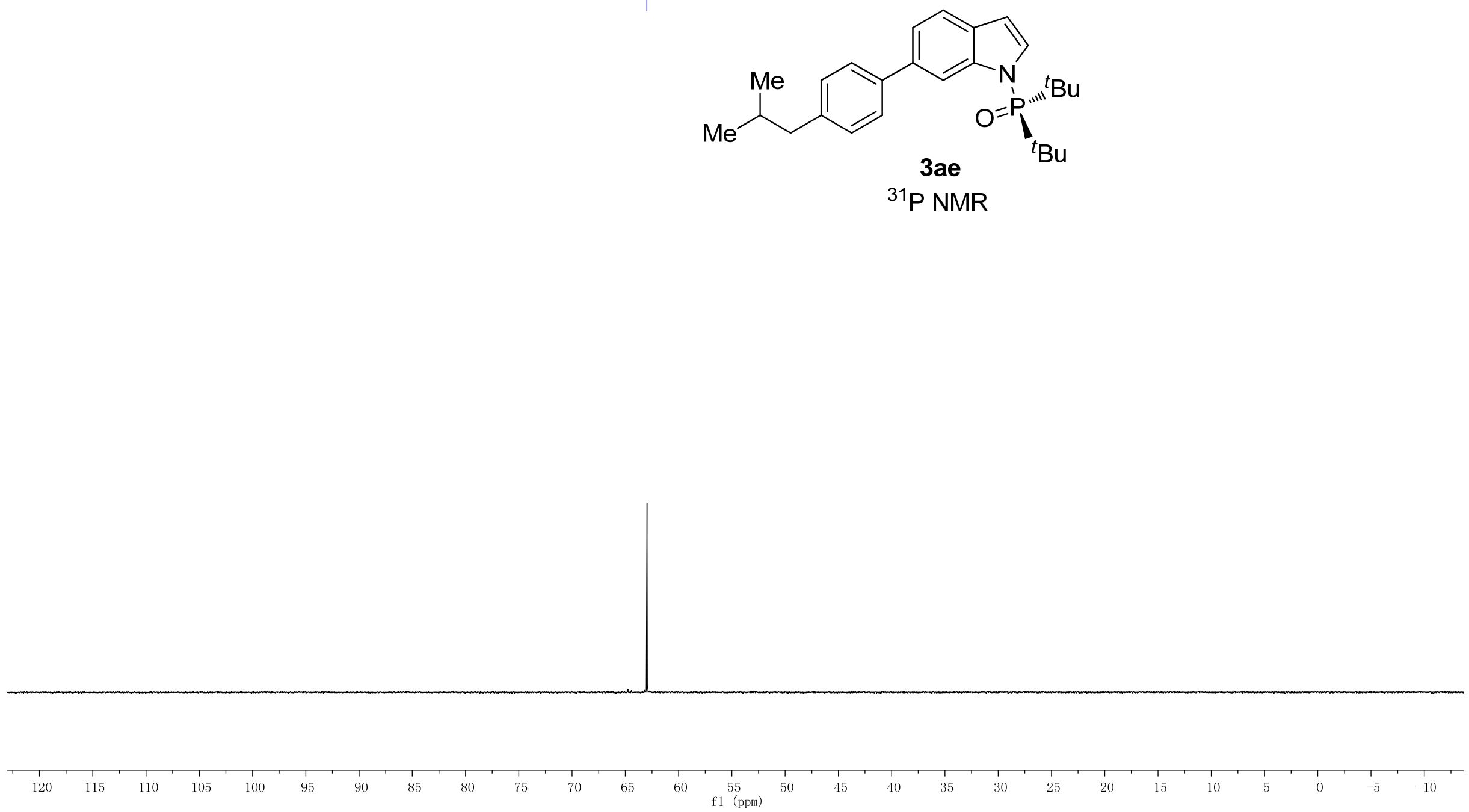

S127 


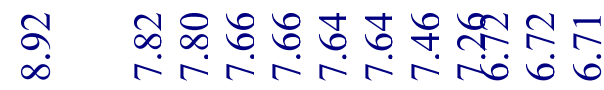

ำ ำ

rryarango

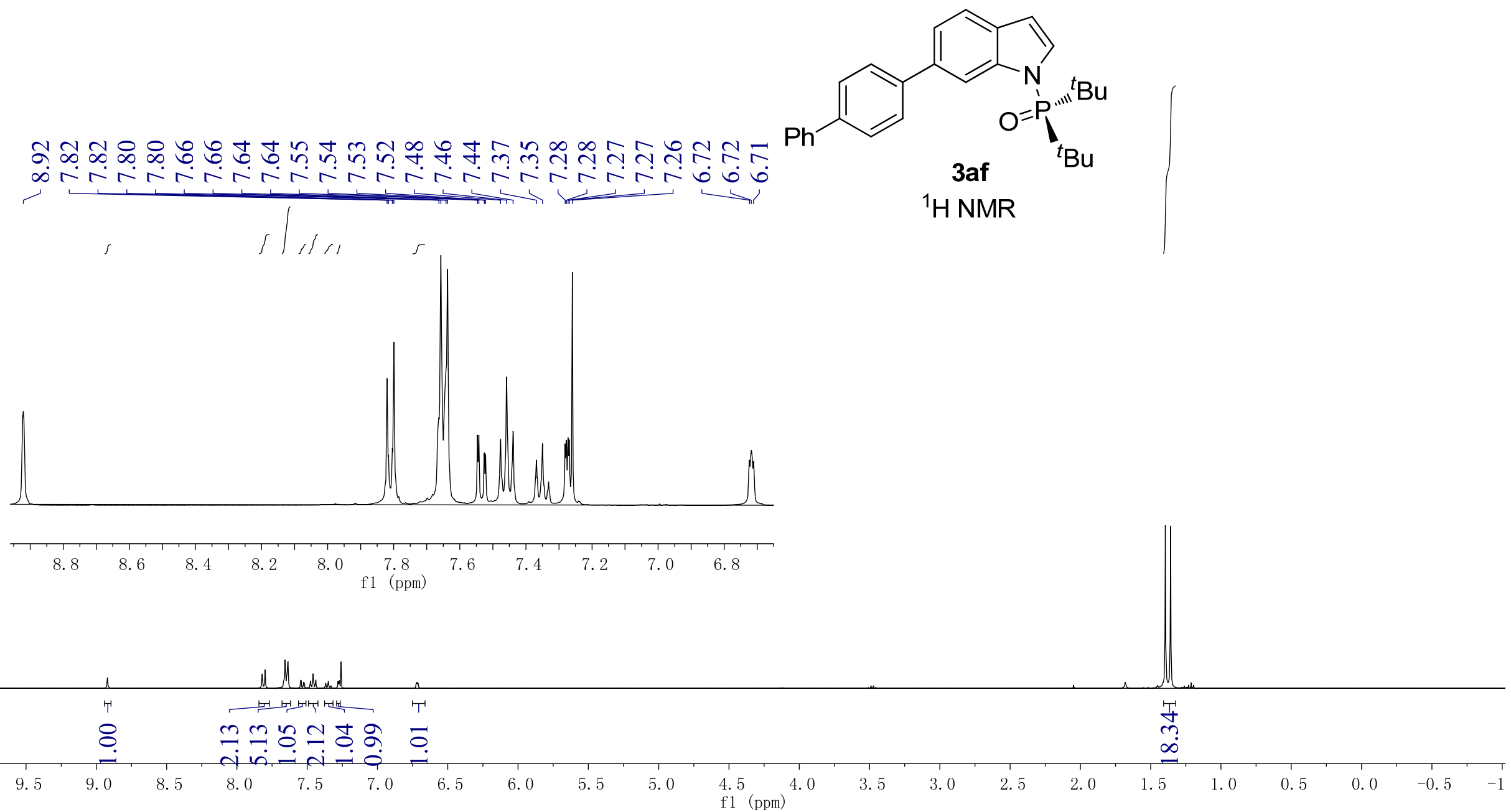




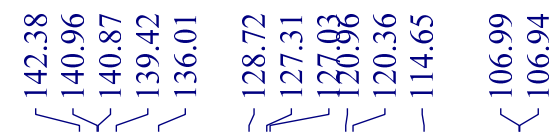

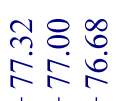

$$
\text { aें }
$$

$\infty$

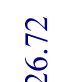

Tr

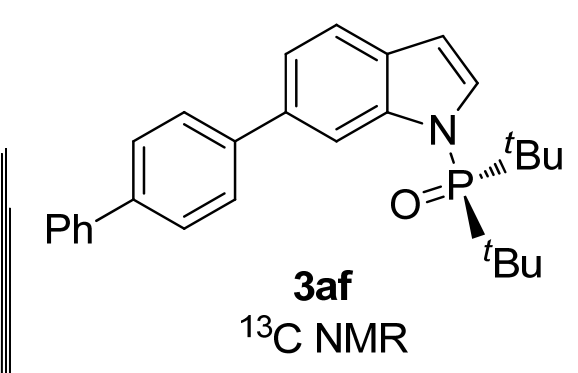

$\begin{array}{llllllllllllllllllllllll}185 & 180 & 175 & 170 & 165 & 160 & 155 & 150 & 145 & 140 & 135 & 130 & 125 & 120 & 115 & 110 & 105 & 100 & 95 & 90 & 8\end{array}$ 
तิ

1
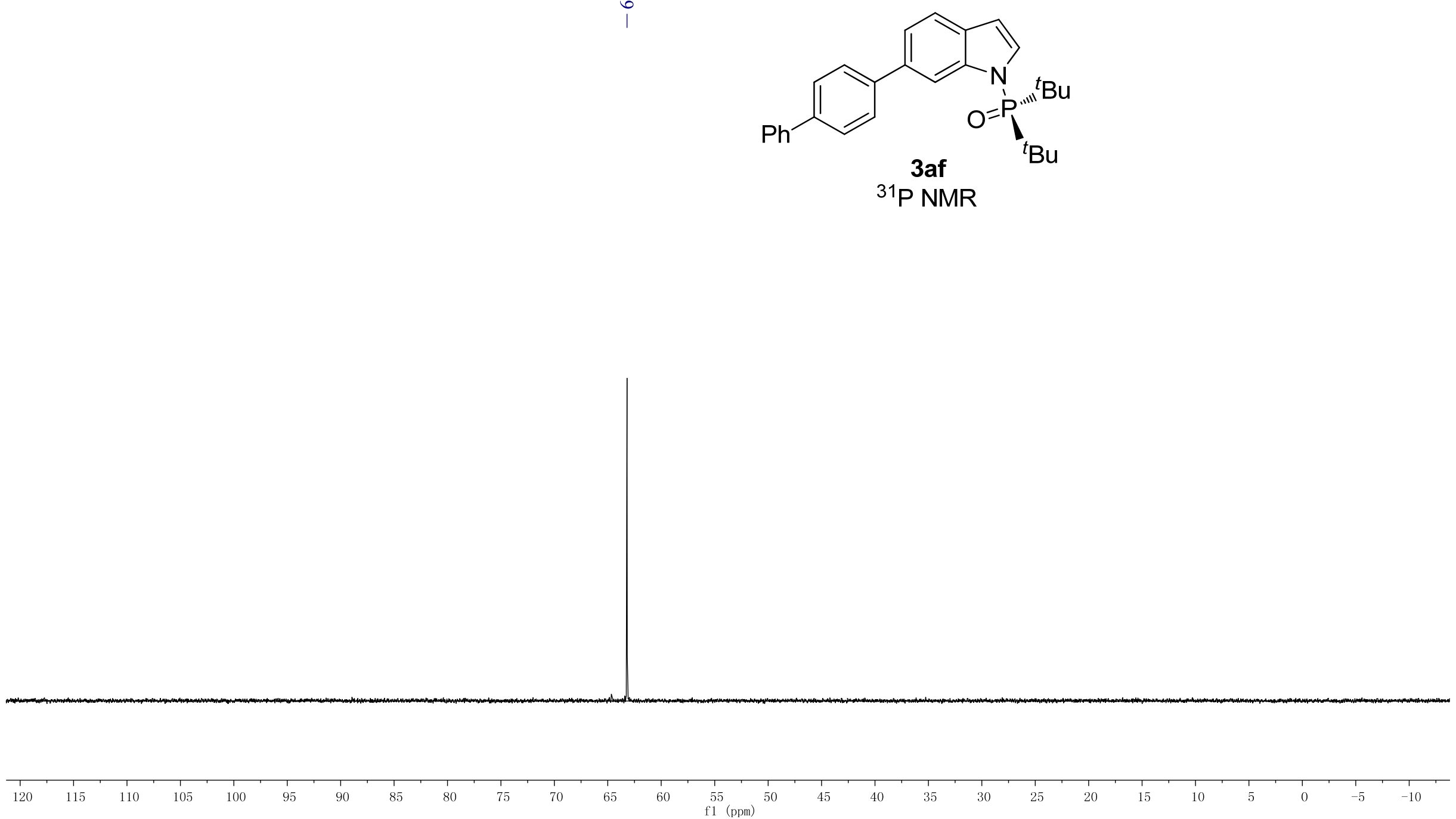


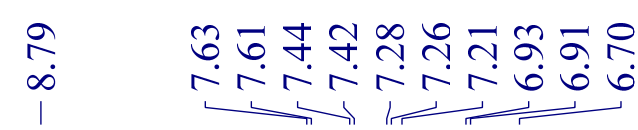

ลे گे

ㅇํำ ำ
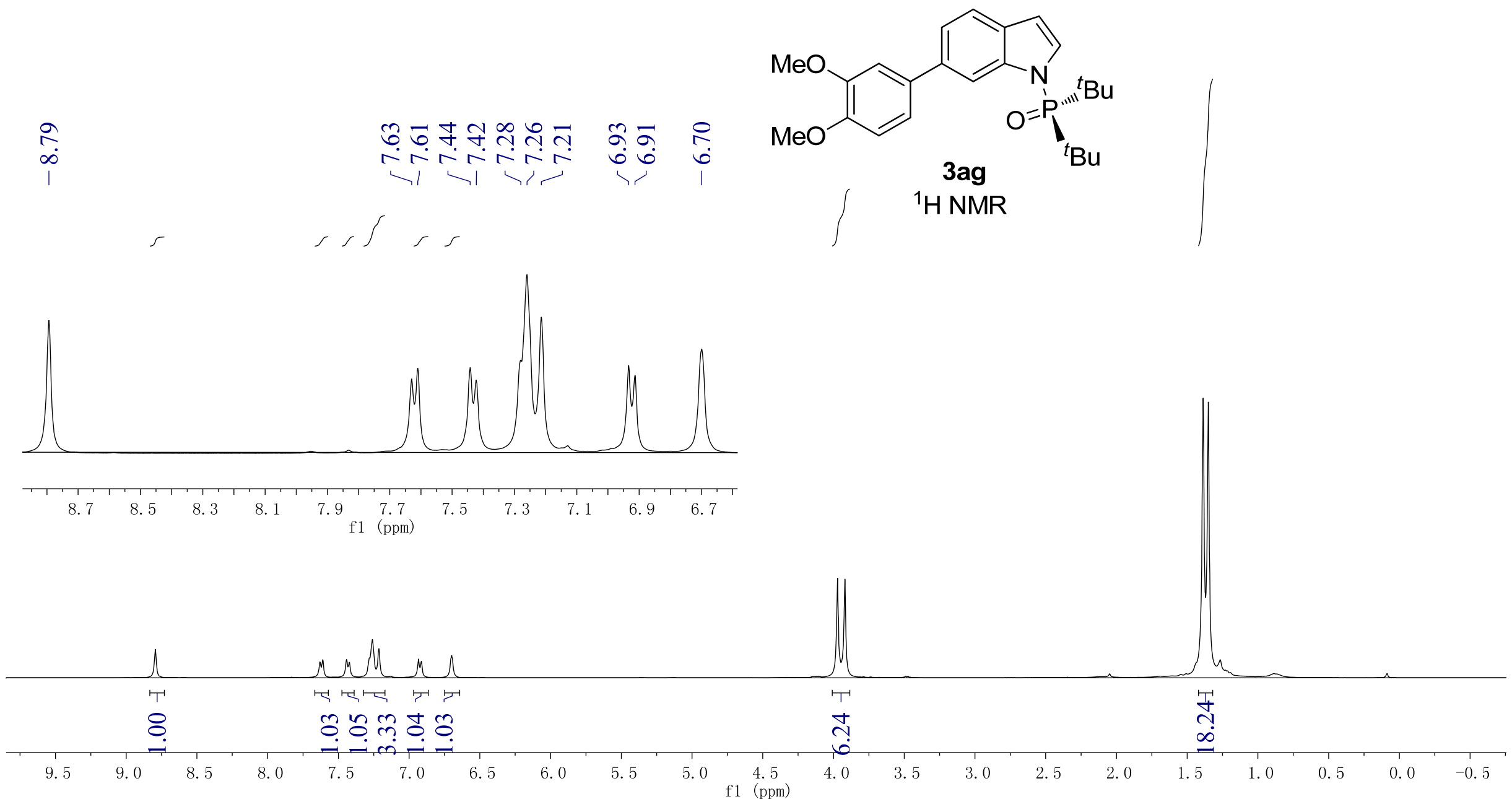


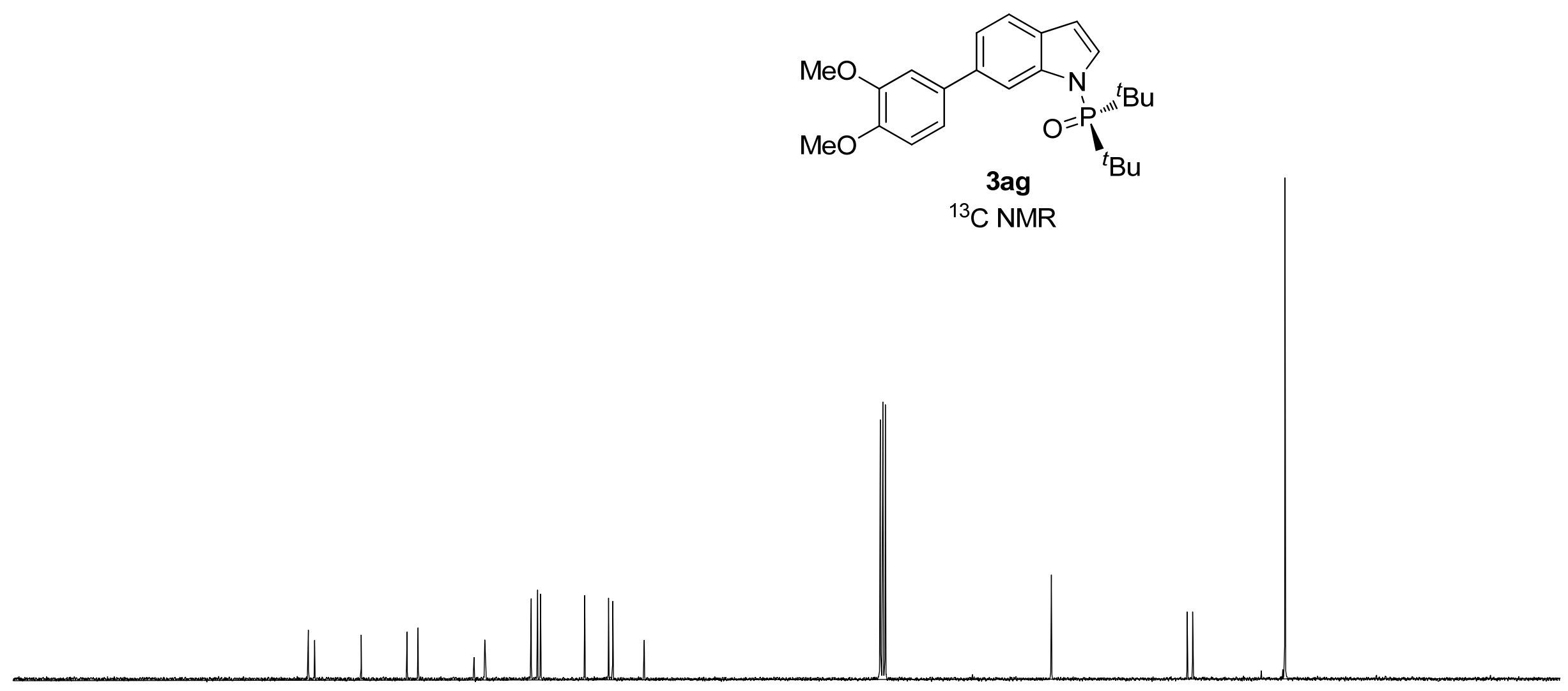

$\begin{array}{llllllllllllllllllllllllll}185 & 180 & 175 & 170 & 165 & 160 & 155 & 150 & 145 & 140 & 135 & 130 & 125 & 120 & 115 & 110 & 105 & 100 & 95 & 90 & 8\end{array}$ 
7
0
1
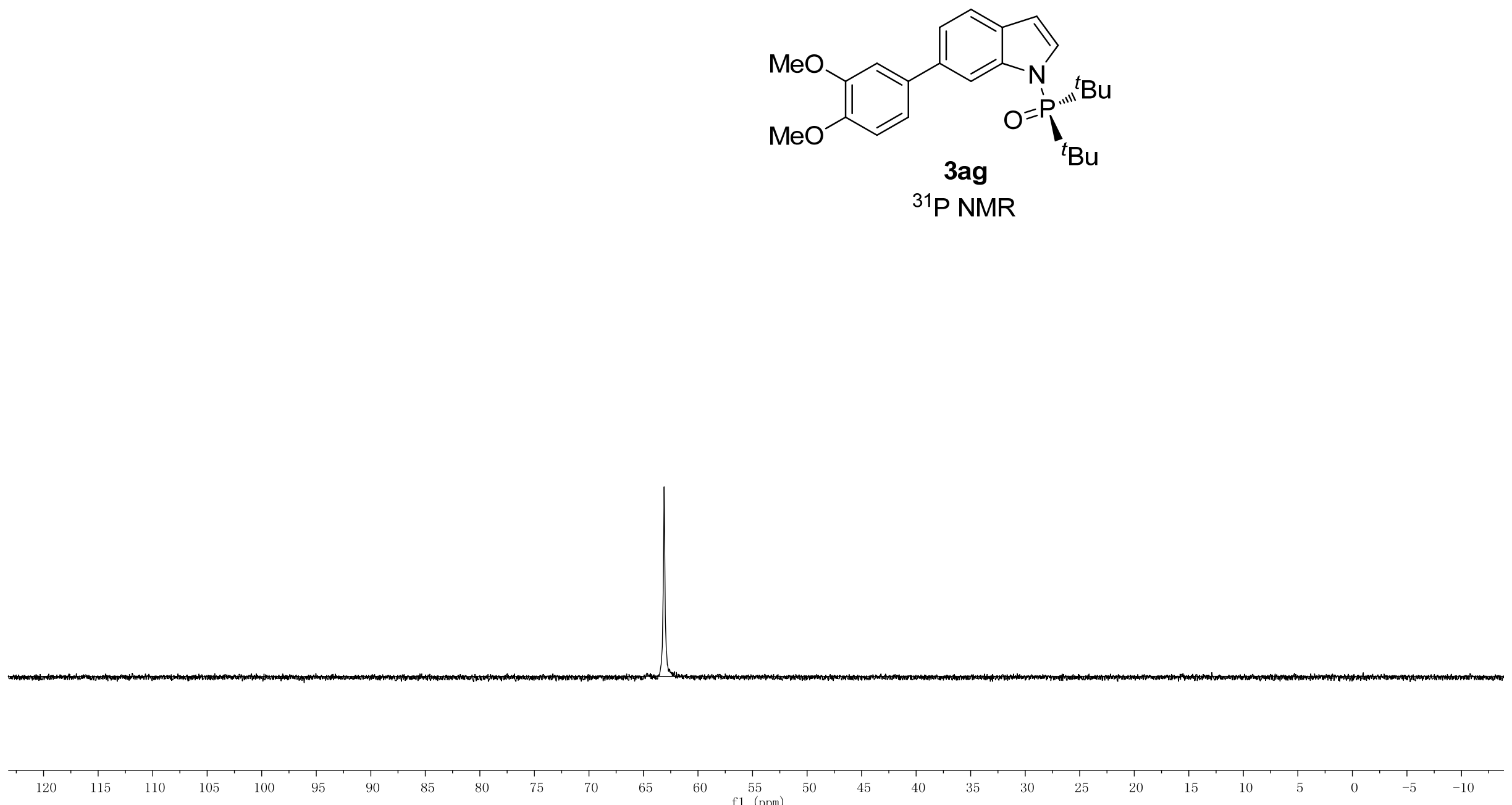
\&

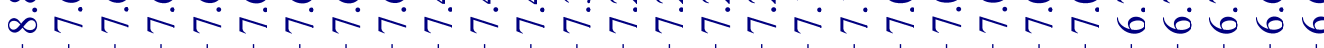

\&
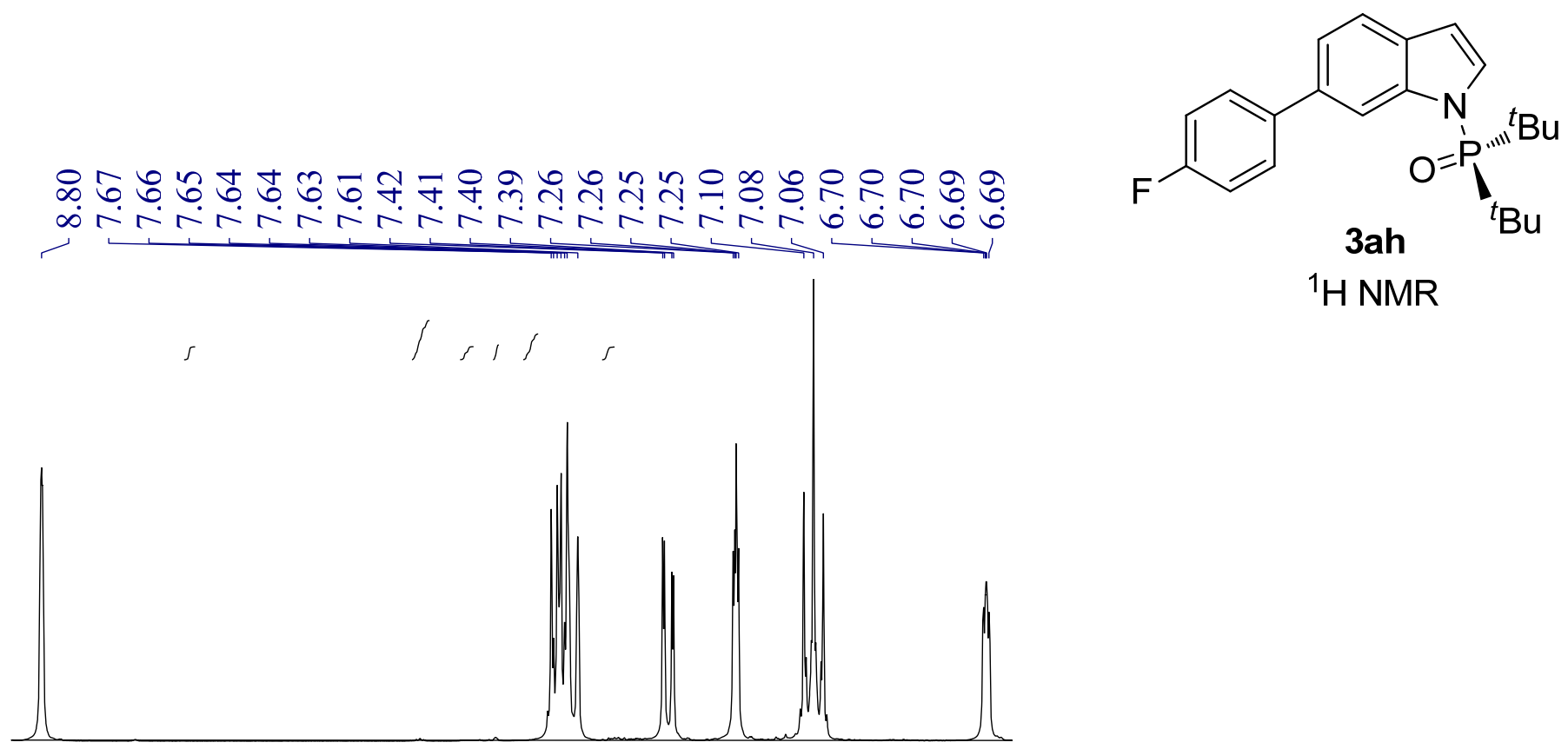

${ }^{1} \mathrm{H}$ NMR
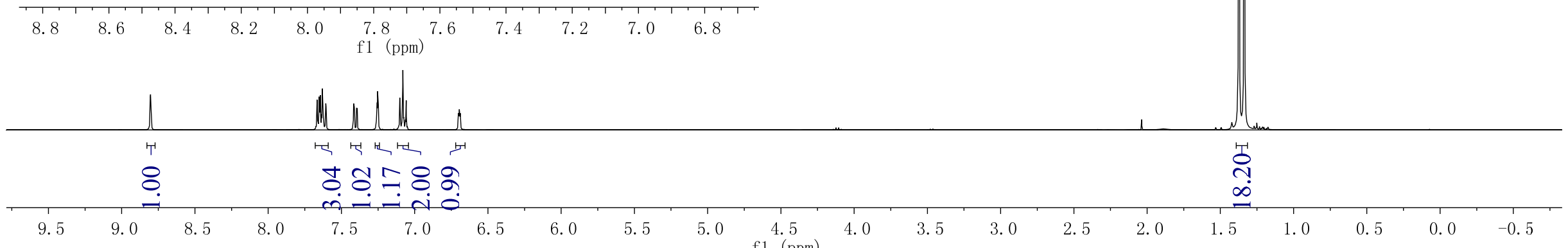


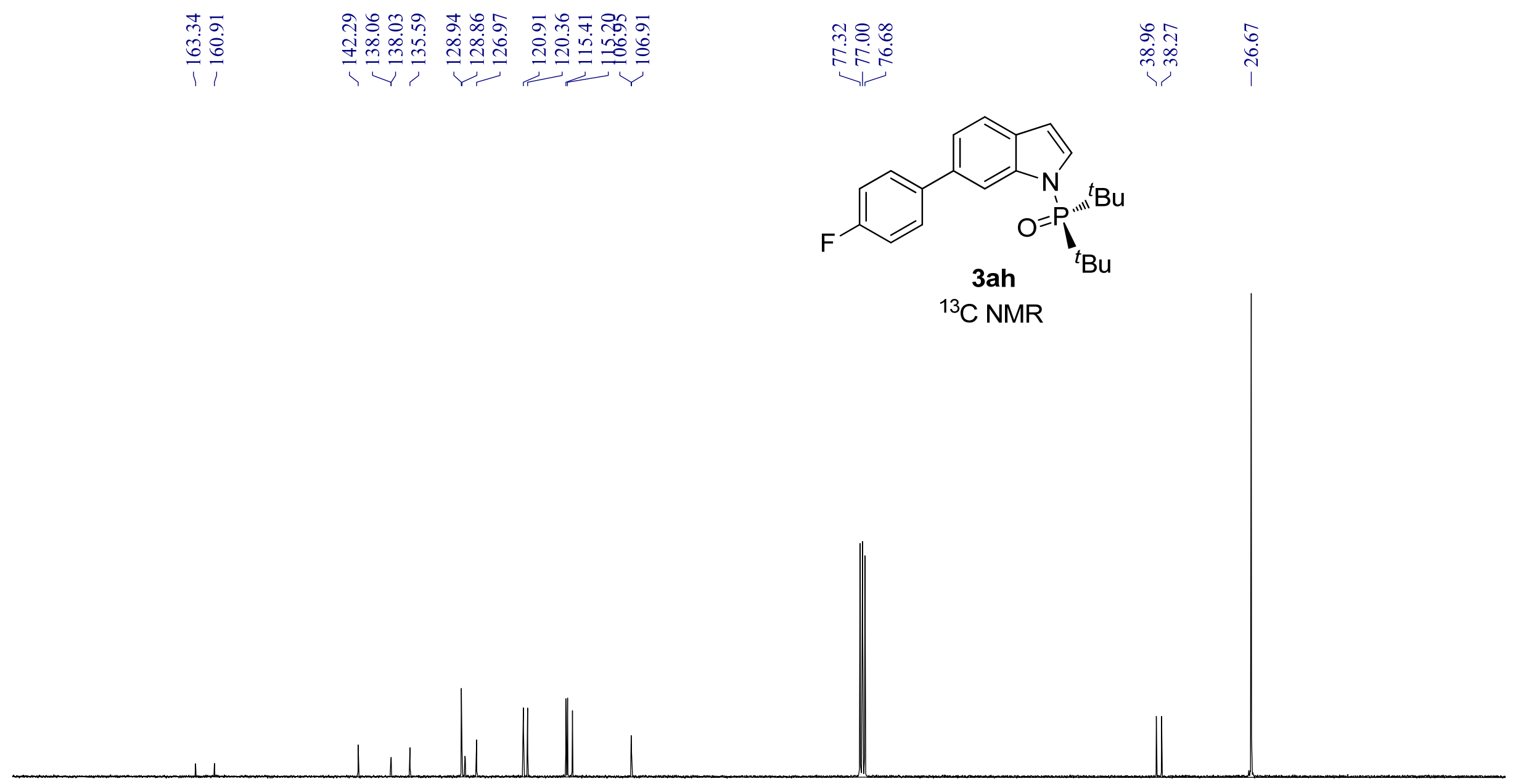

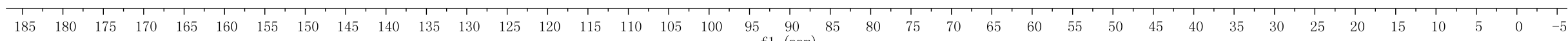


ले

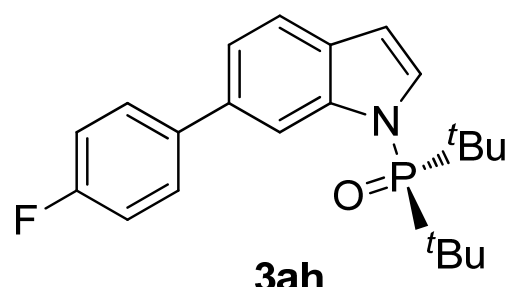

${ }^{31} \mathrm{P}$ NMR

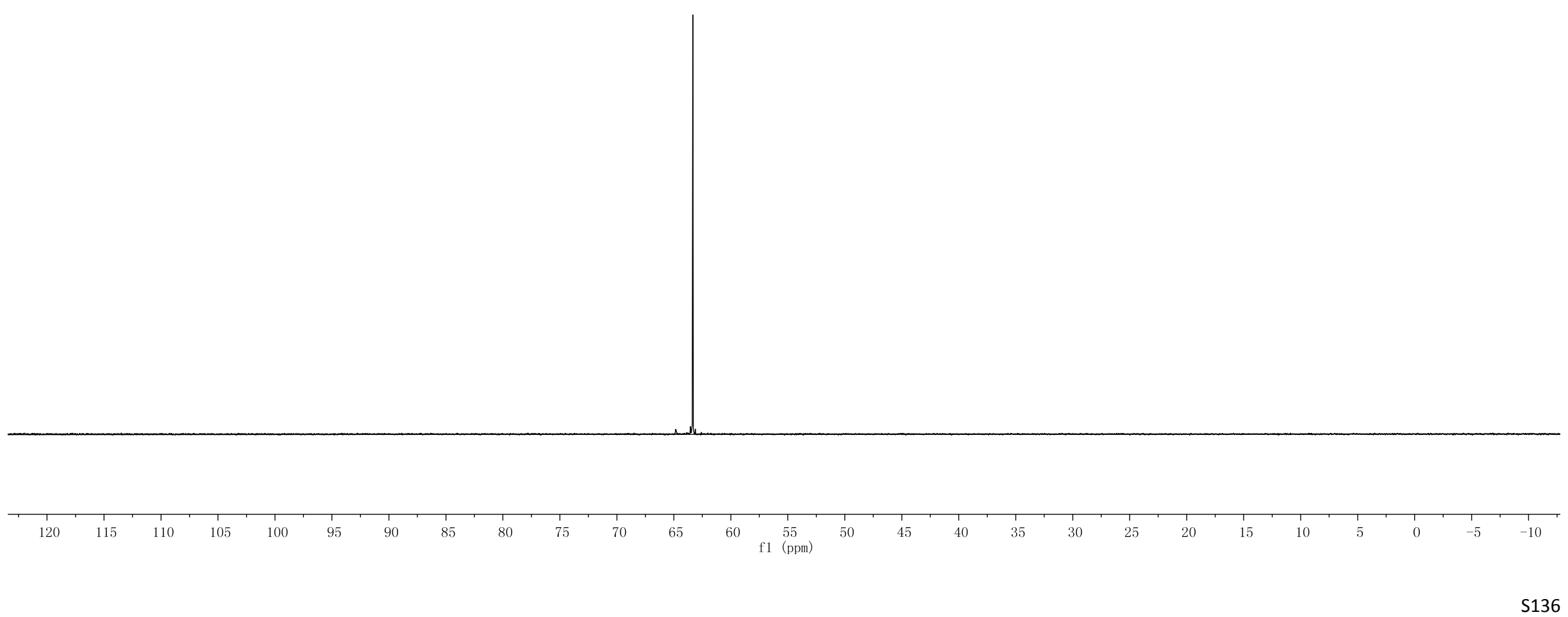



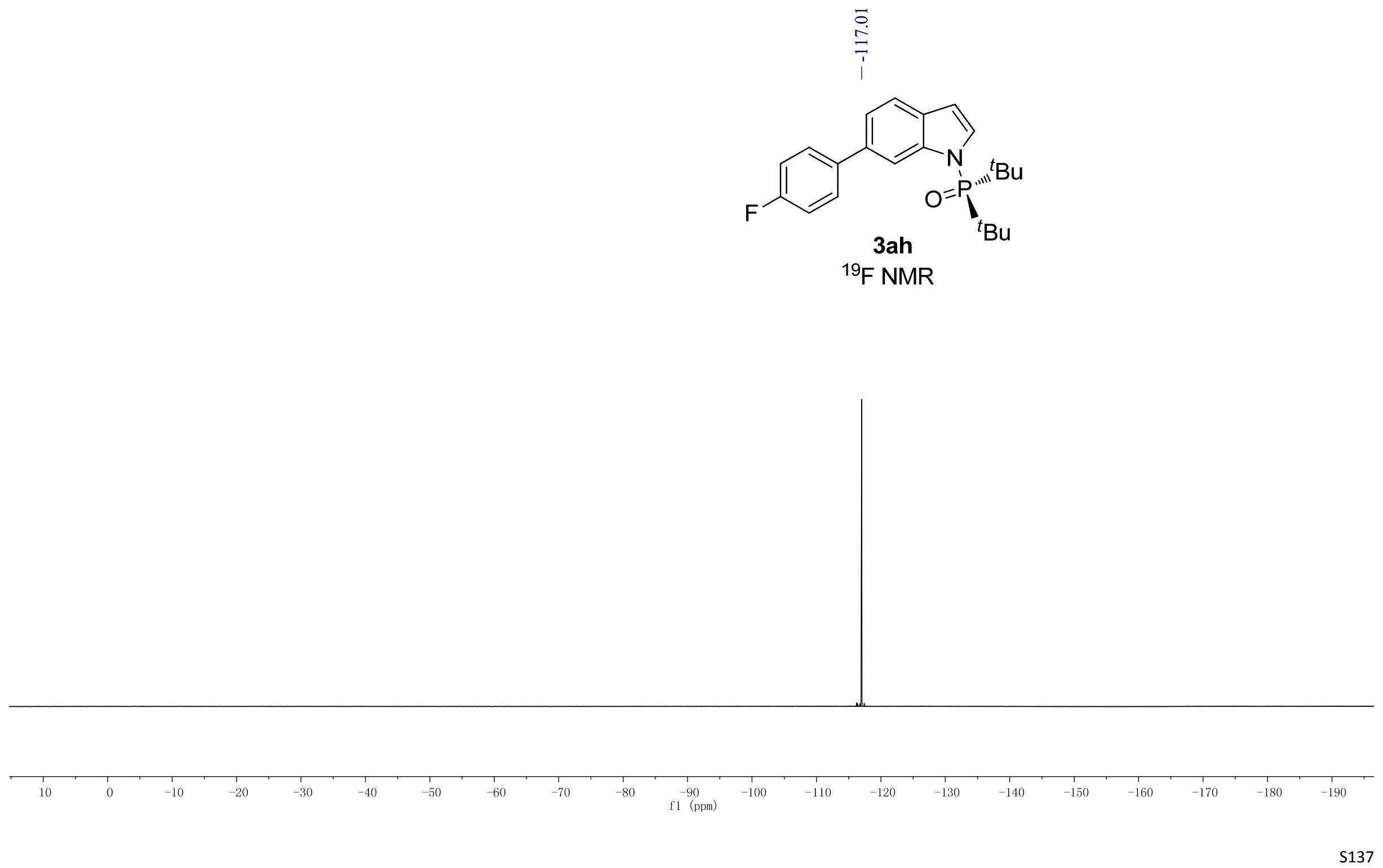


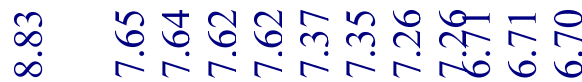

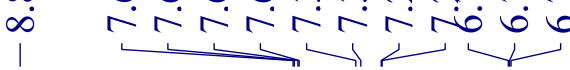

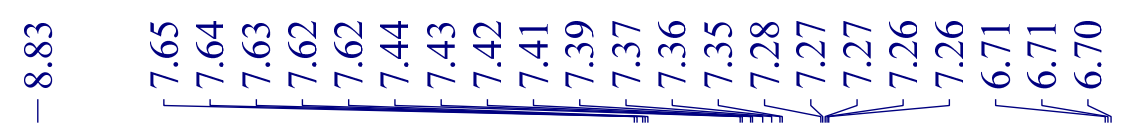
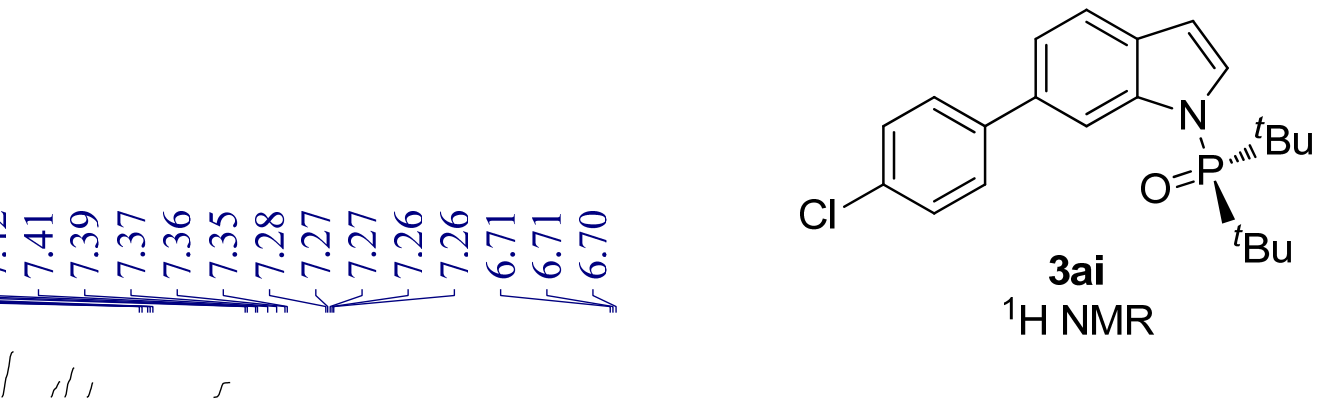

3 ai

${ }^{1} \mathrm{H} N M R$

$\stackrel{\infty}{\stackrel{4}{9}}$

\)
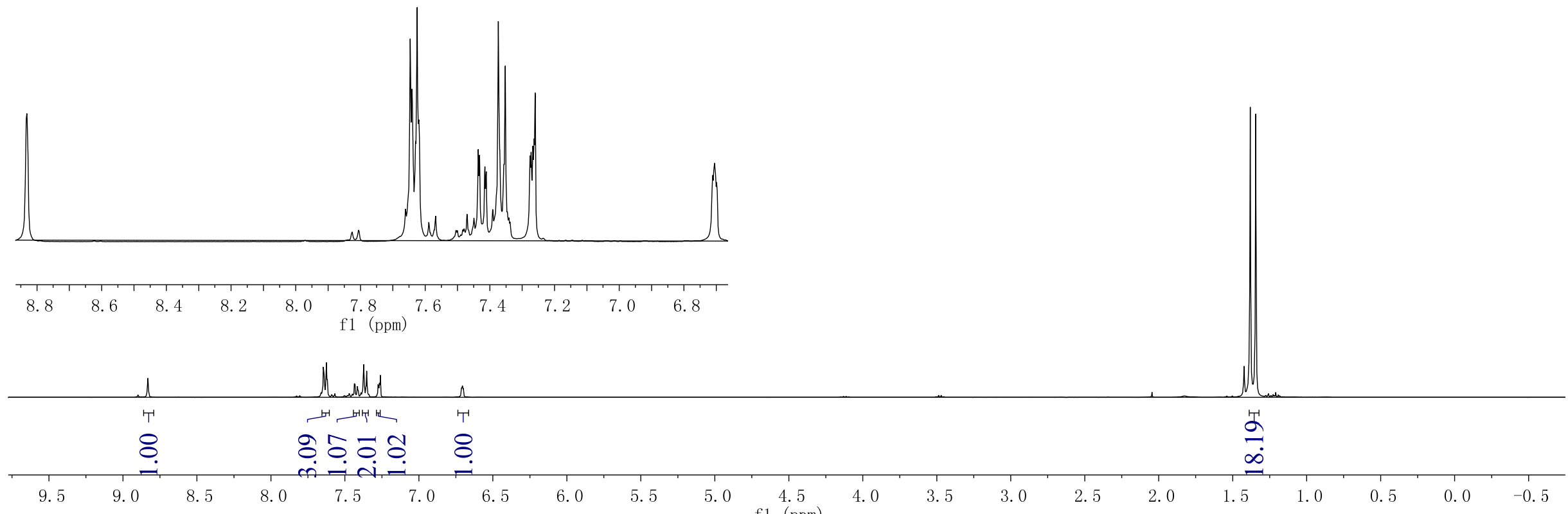

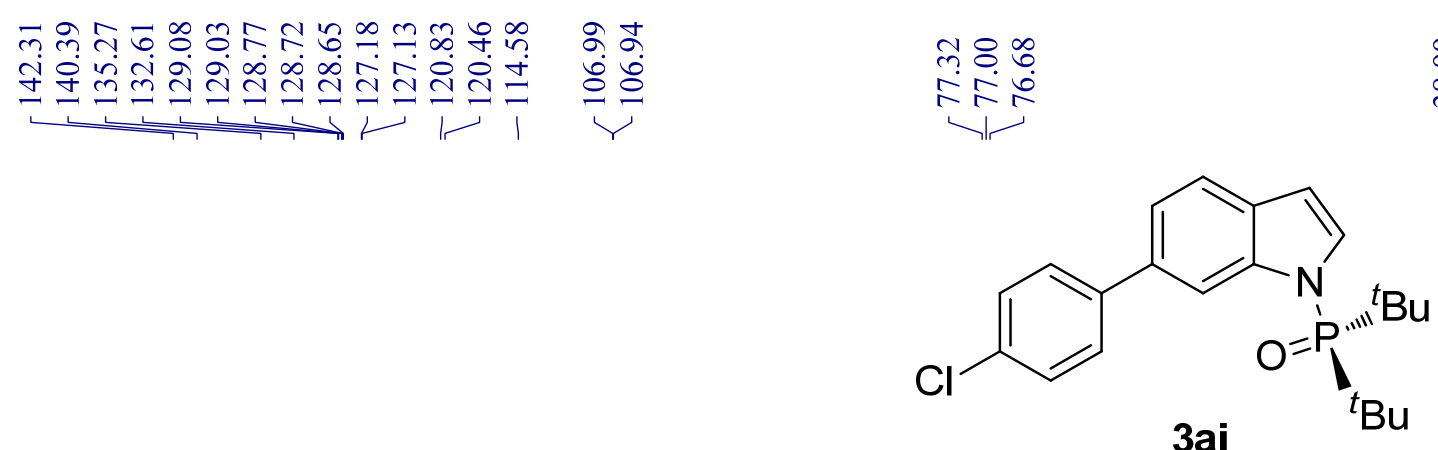

${ }^{13}$ C NMR

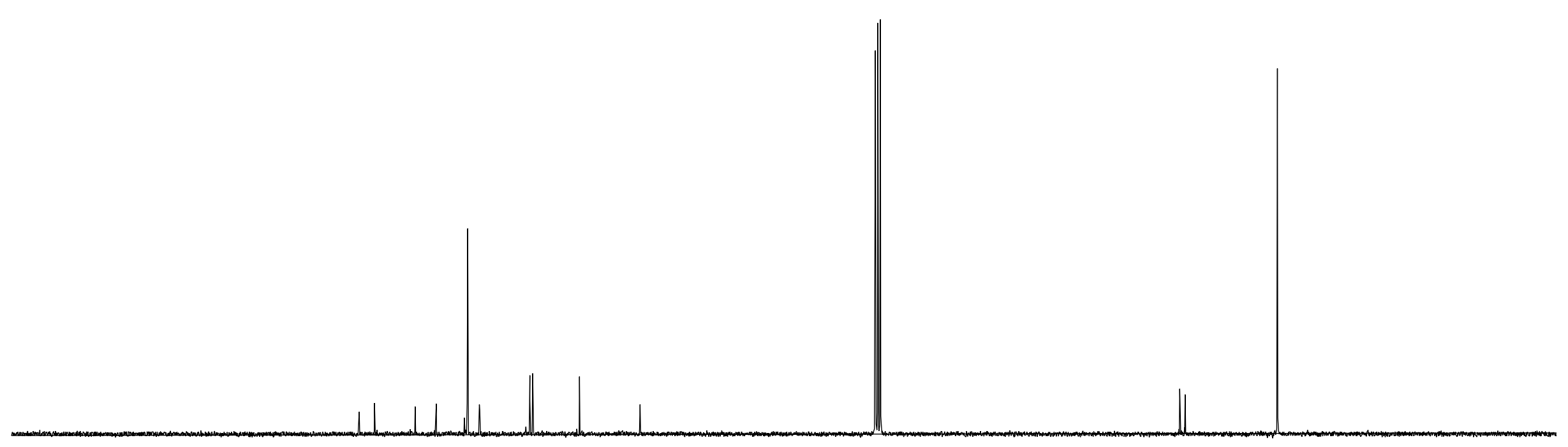

$\begin{array}{llllllllllllllllllllll}185 & 180 & 175 & 170 & 165 & 160 & 155 & 150 & 145 & 140 & 135 & 130 & 125 & 120 & 115 & 110 & 105 & 100 & 95 & 90 & 85\end{array}$ 
$\stackrel{y}{\vartheta}$
ชุ
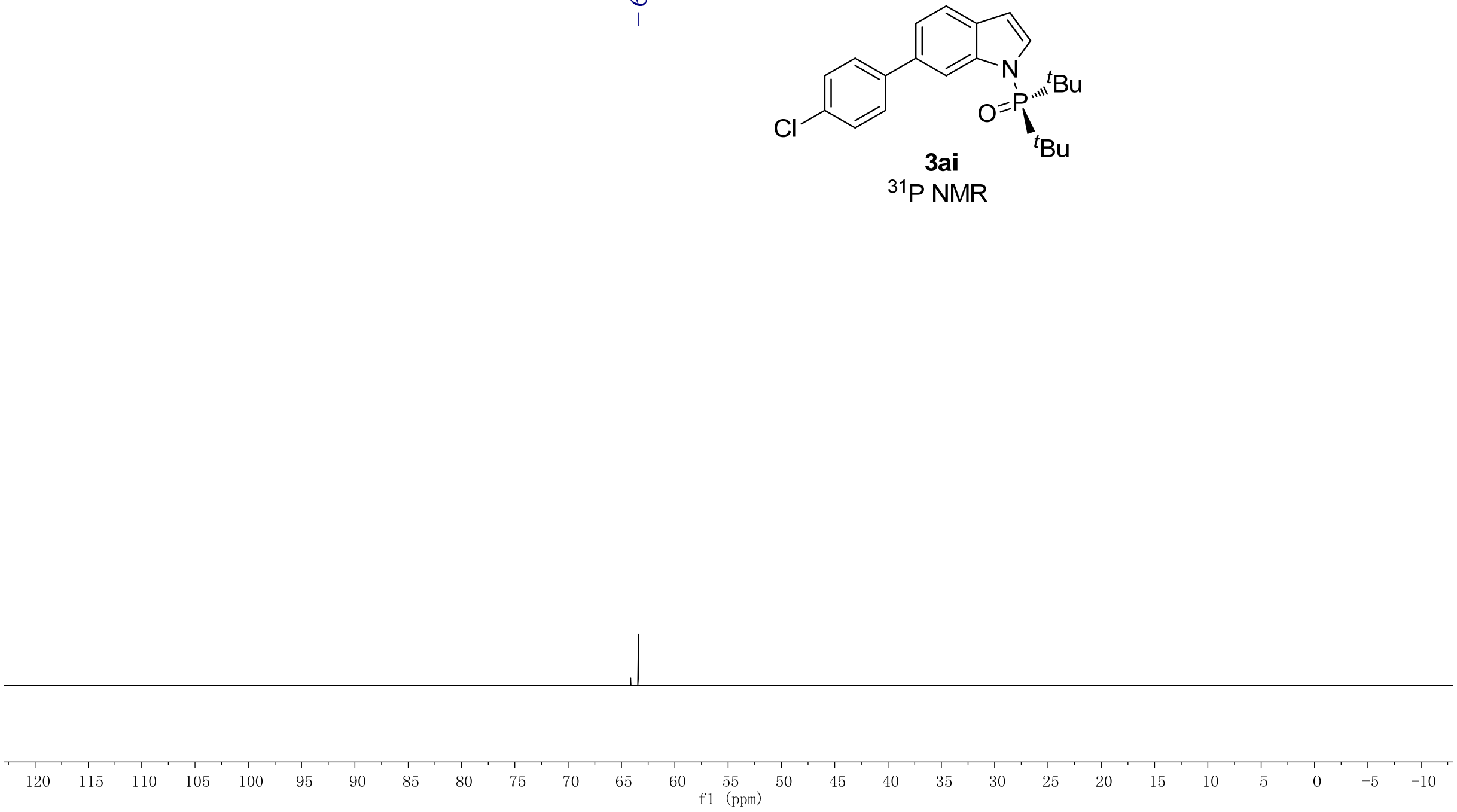
क.

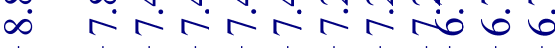

- ๙ o 0 .
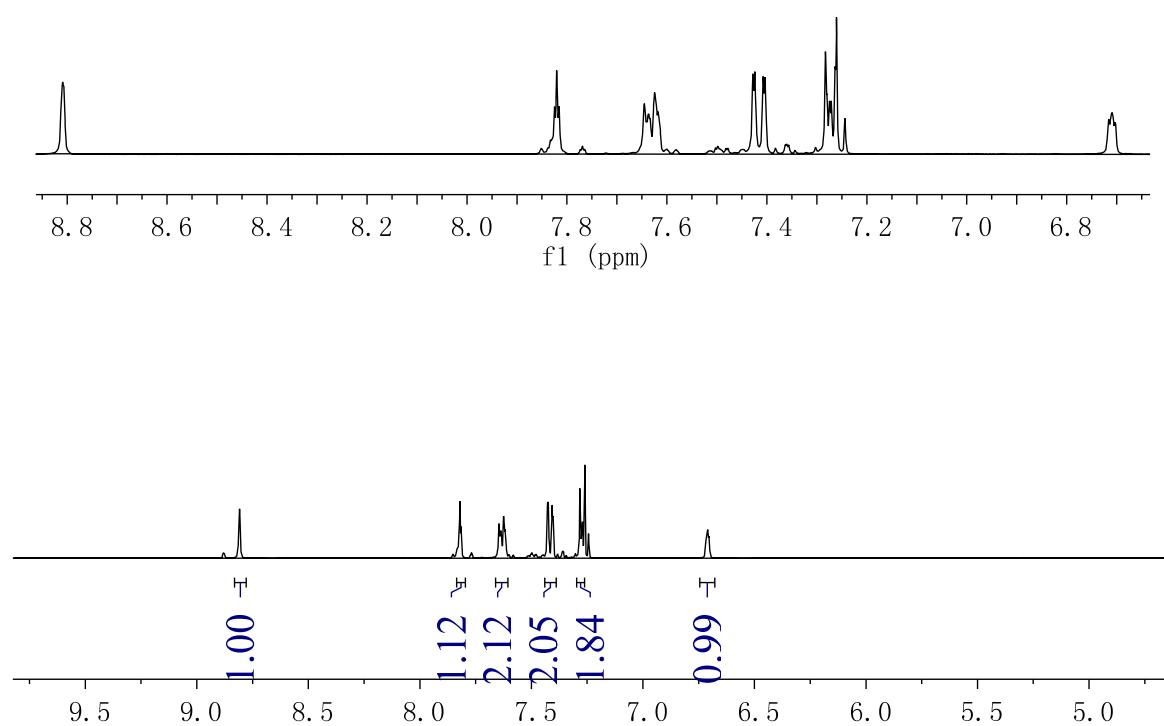

m?

T)

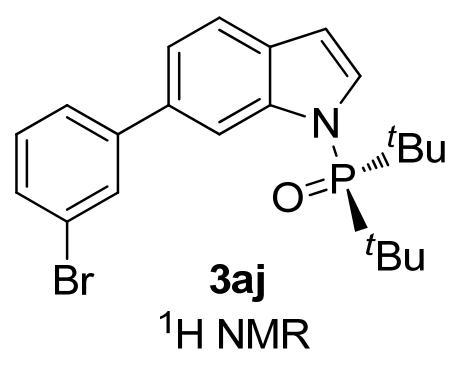




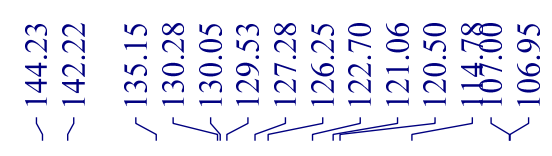

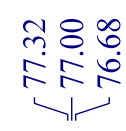

$\begin{array}{ll}2 \pi & \text { aे } \\ \infty & \infty \\ \infty & \text { i }\end{array}$
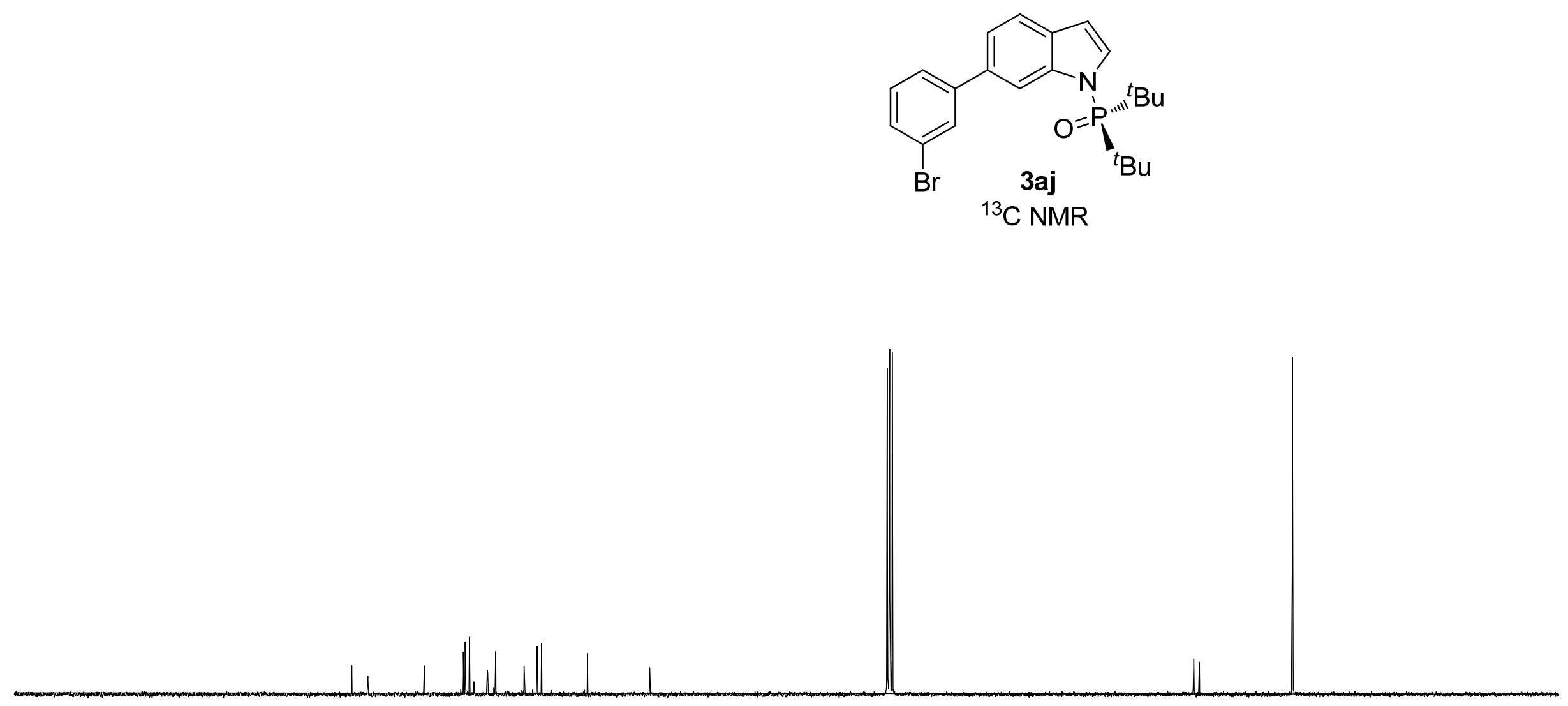

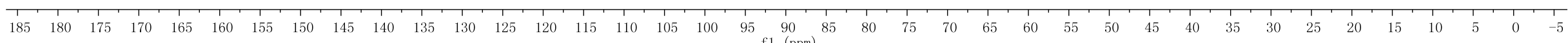


$n$
$n$
0
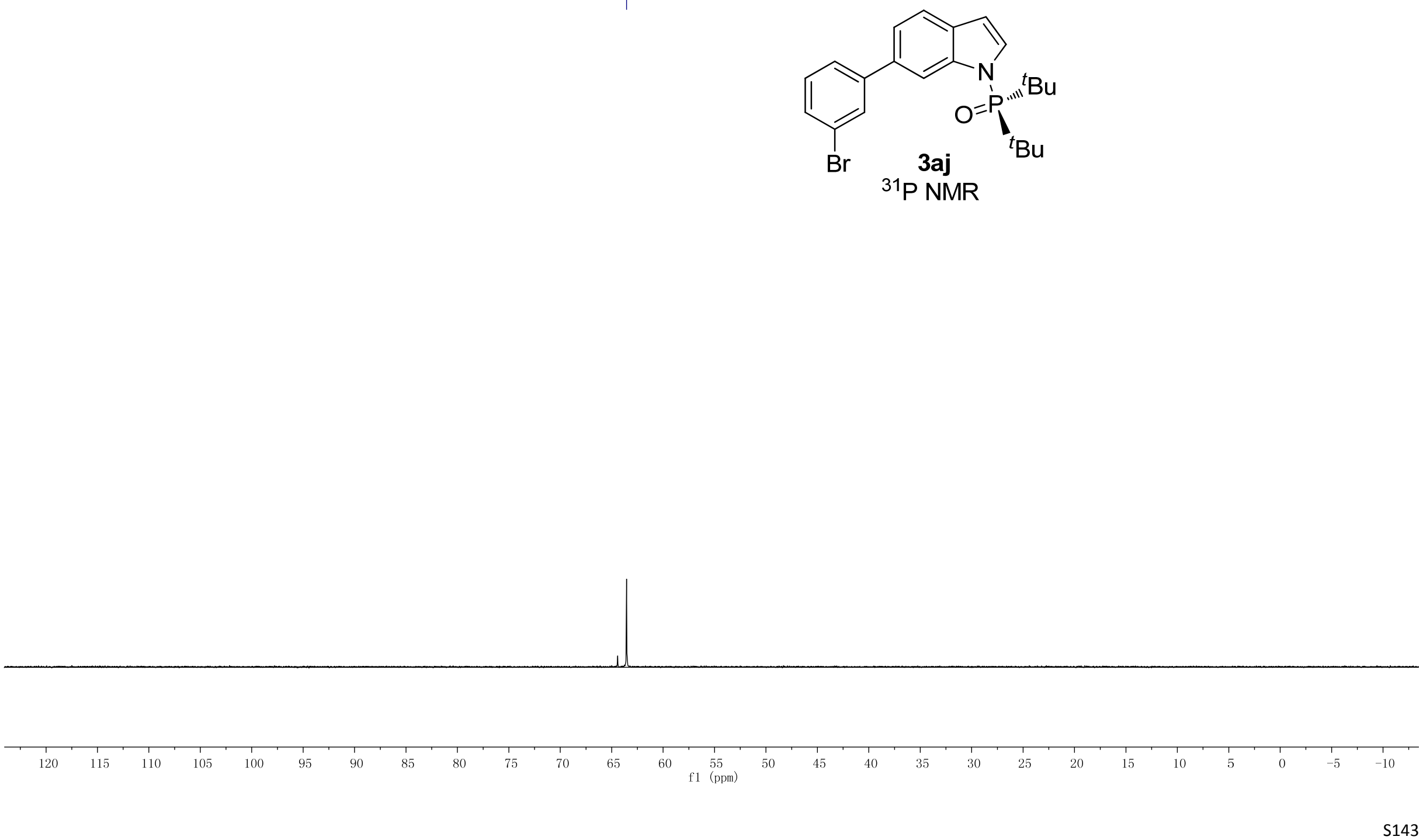


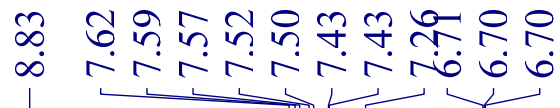

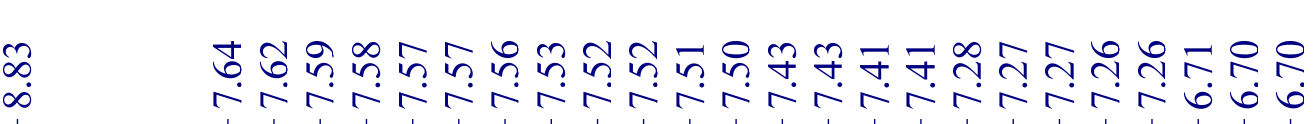
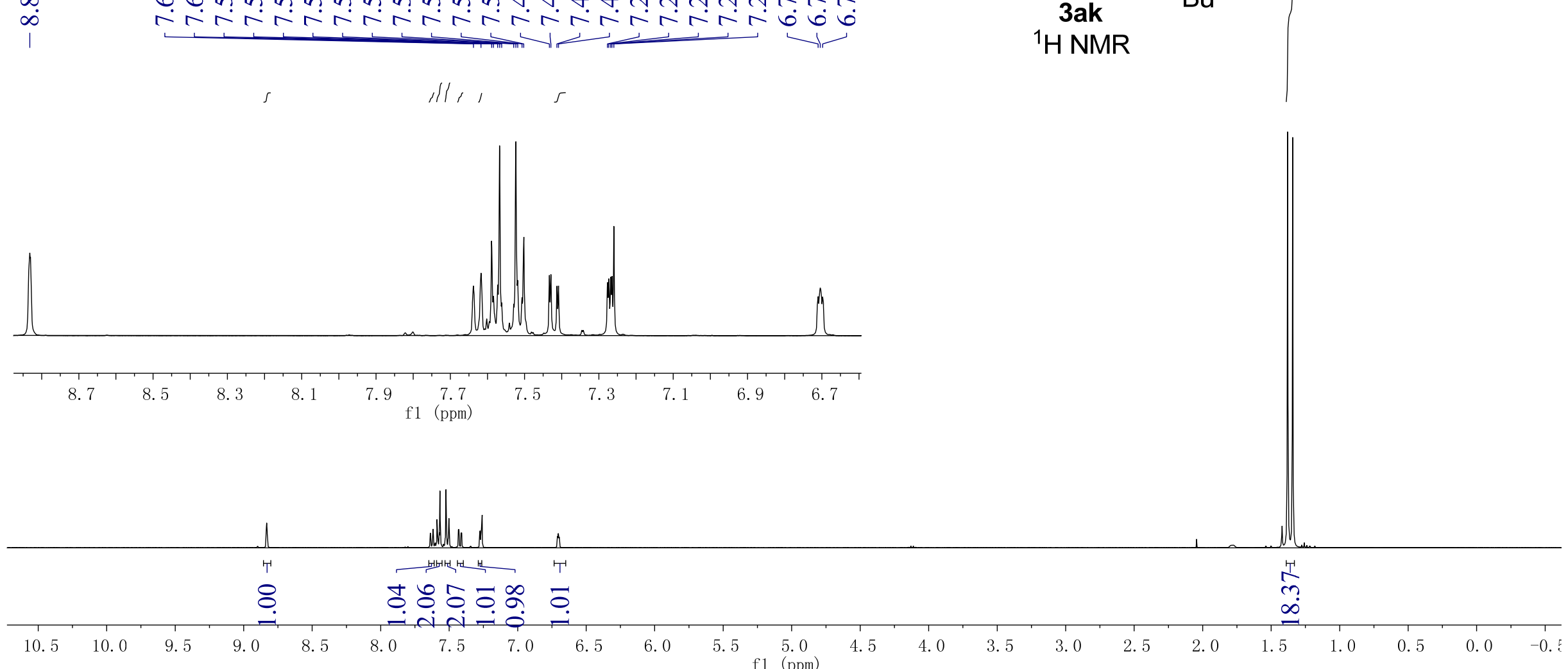


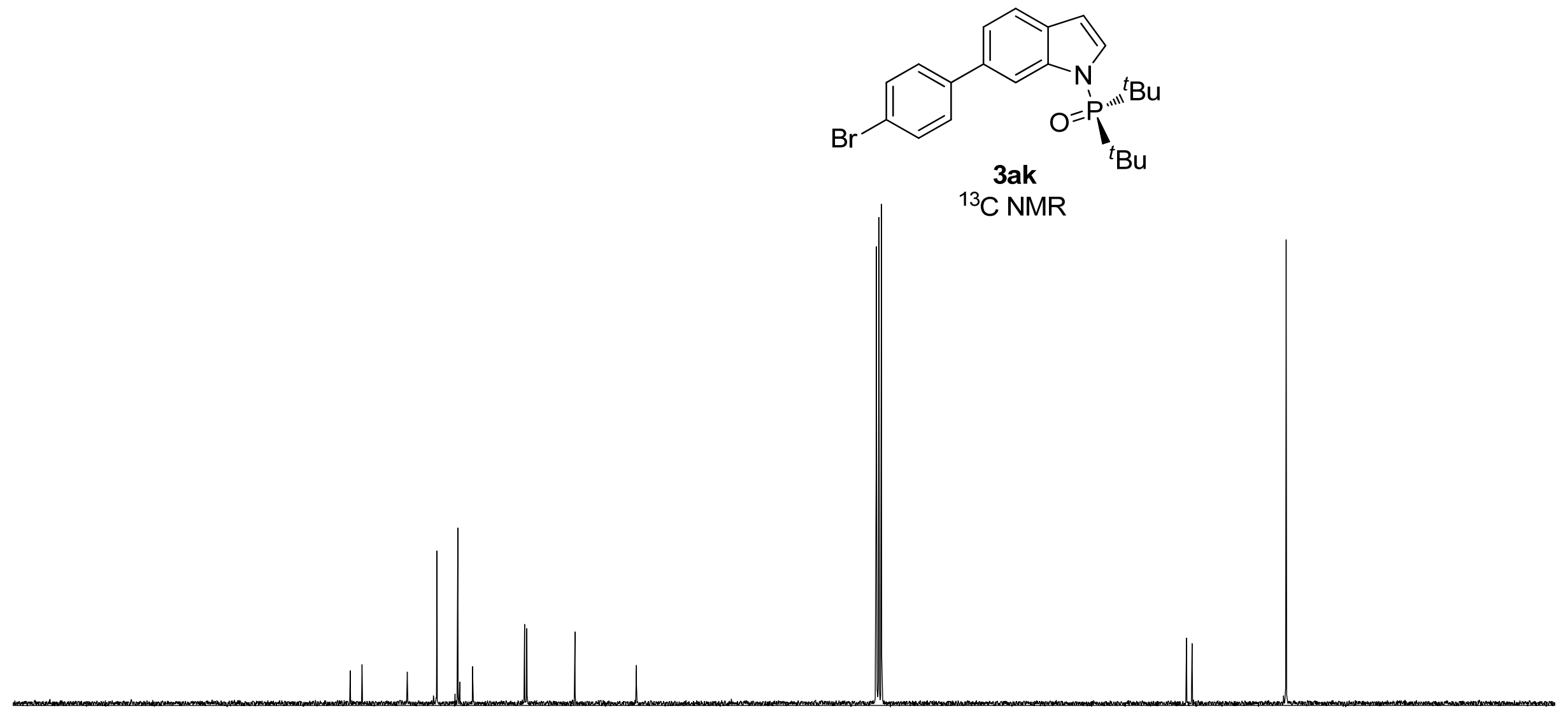

$\begin{array}{llllllllllllllllllll}180 & 175 & 170 & 165 & 160 & 155 & 150 & 145 & 140 & 135 & 130 & 125 & 120 & 115 & 110 & 105 & 100 & 95 & 90 & 8\end{array}$ 
\%)

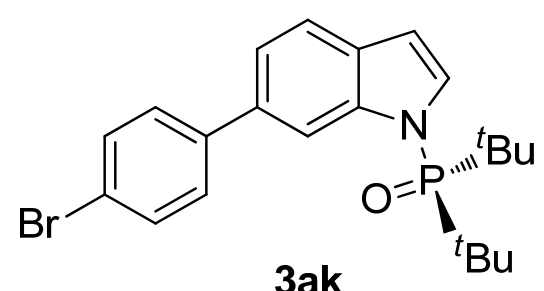

${ }^{31} \mathrm{P}$ NMR

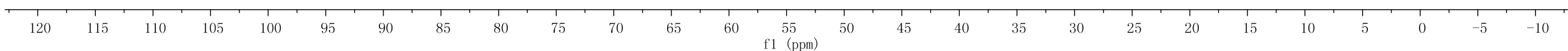


n.

i rinisivingo

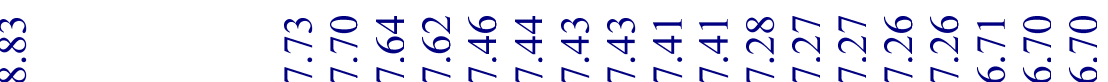

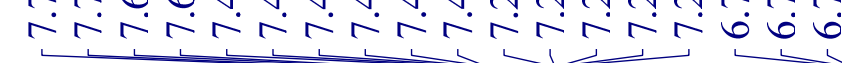

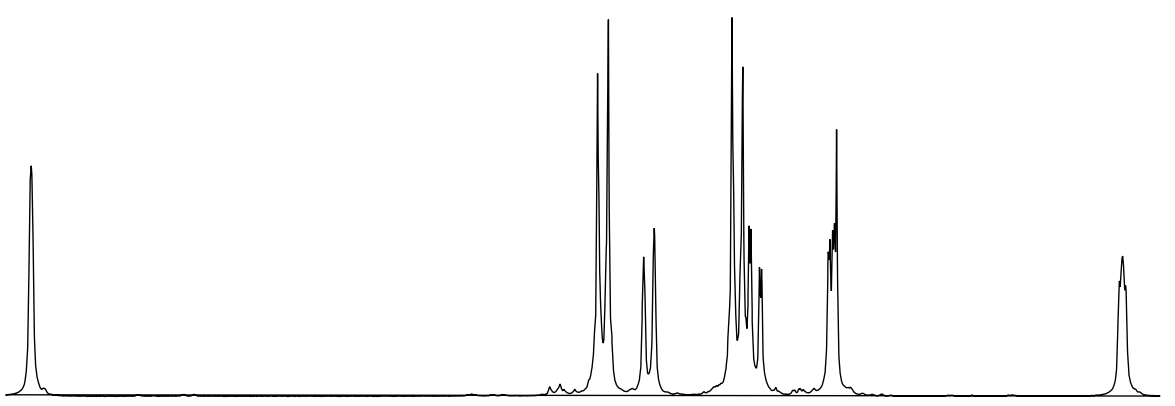

ले

ᄂ

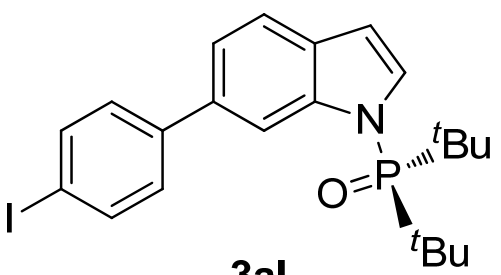

${ }^{1} \mathrm{H}$ NMR

${ }^{t} \mathrm{Bu}$

HNR
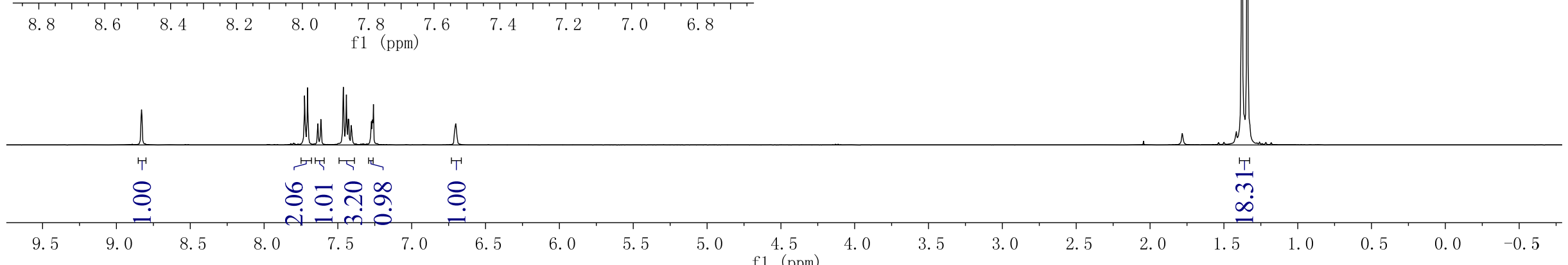

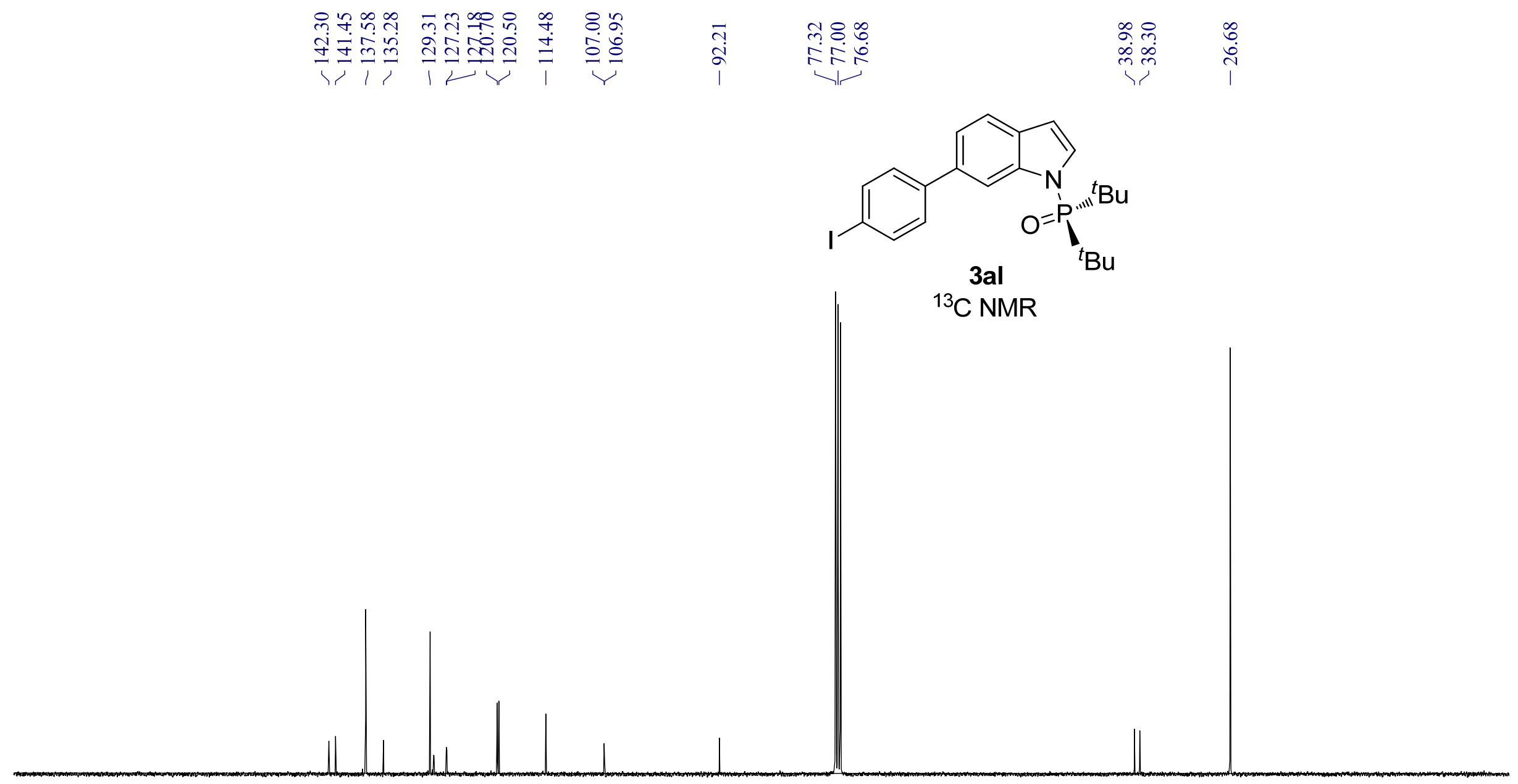

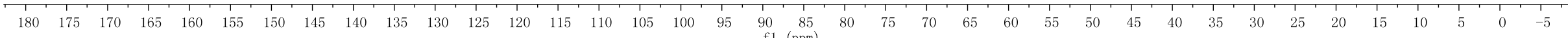


ๆ.
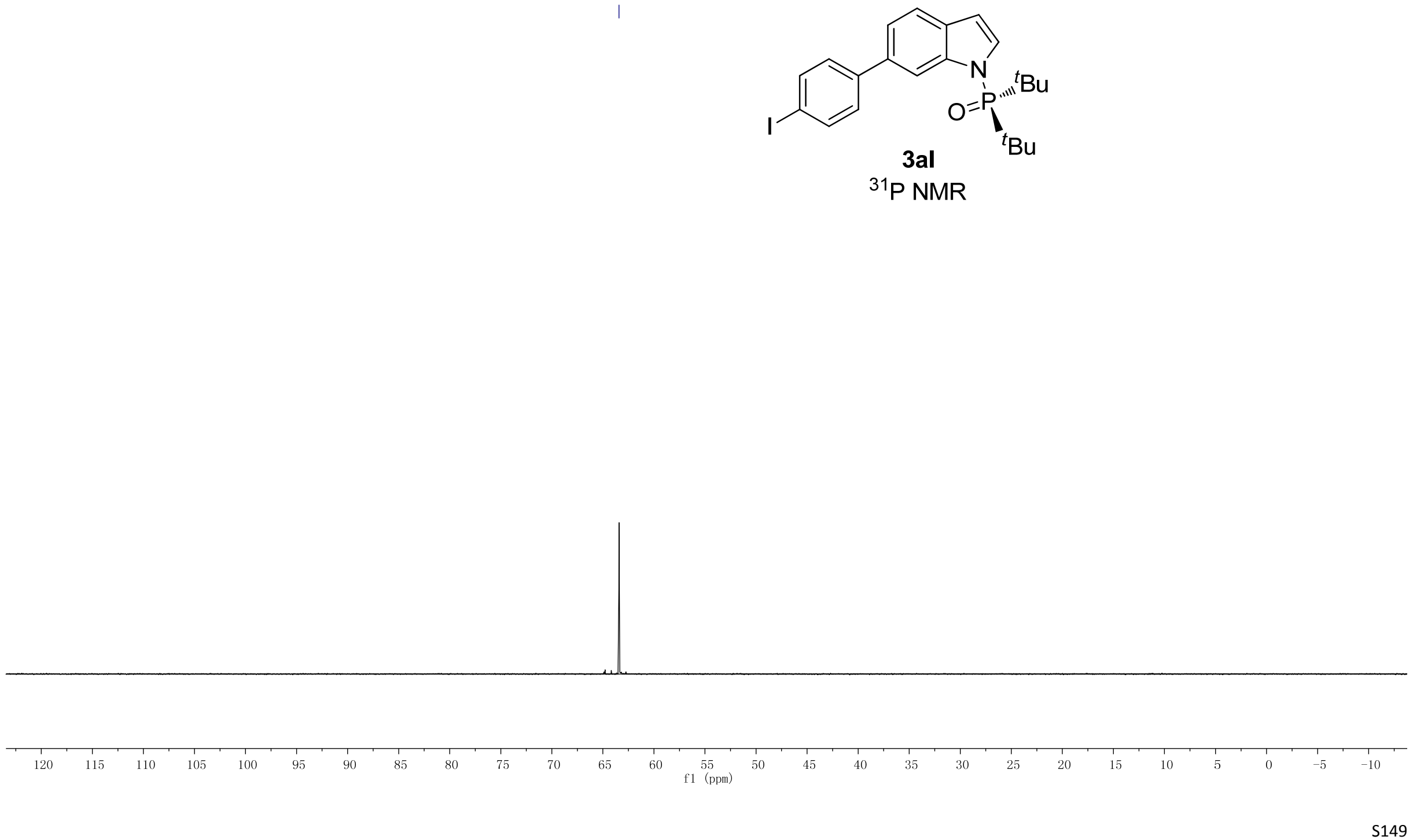


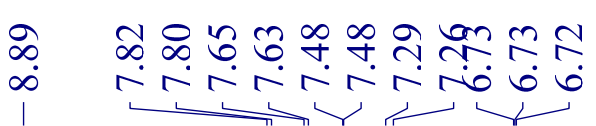
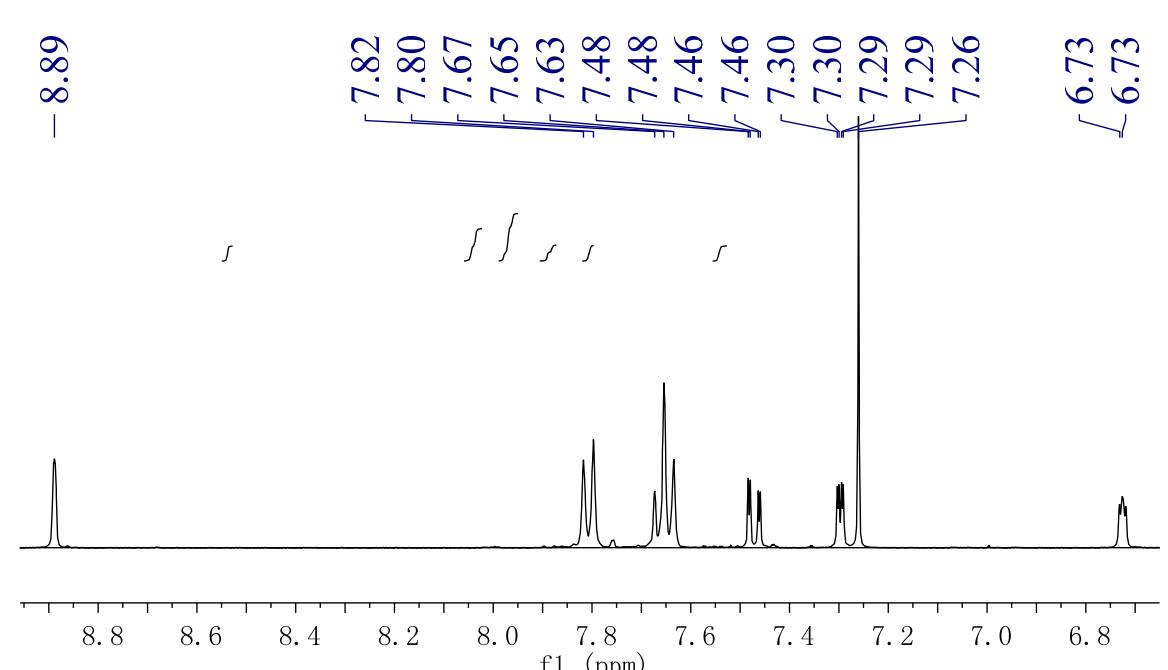

f1 (ppm)

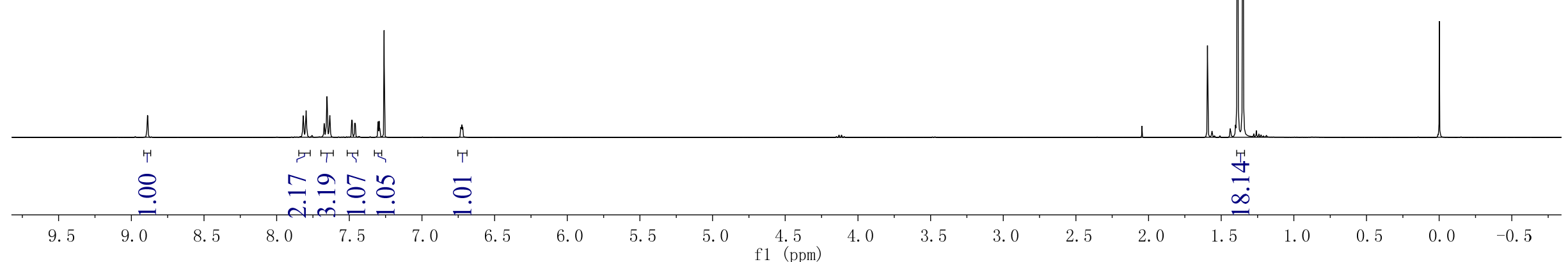

ํำำ

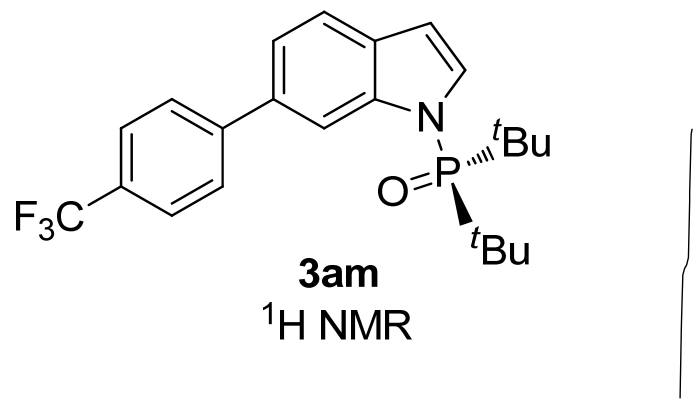




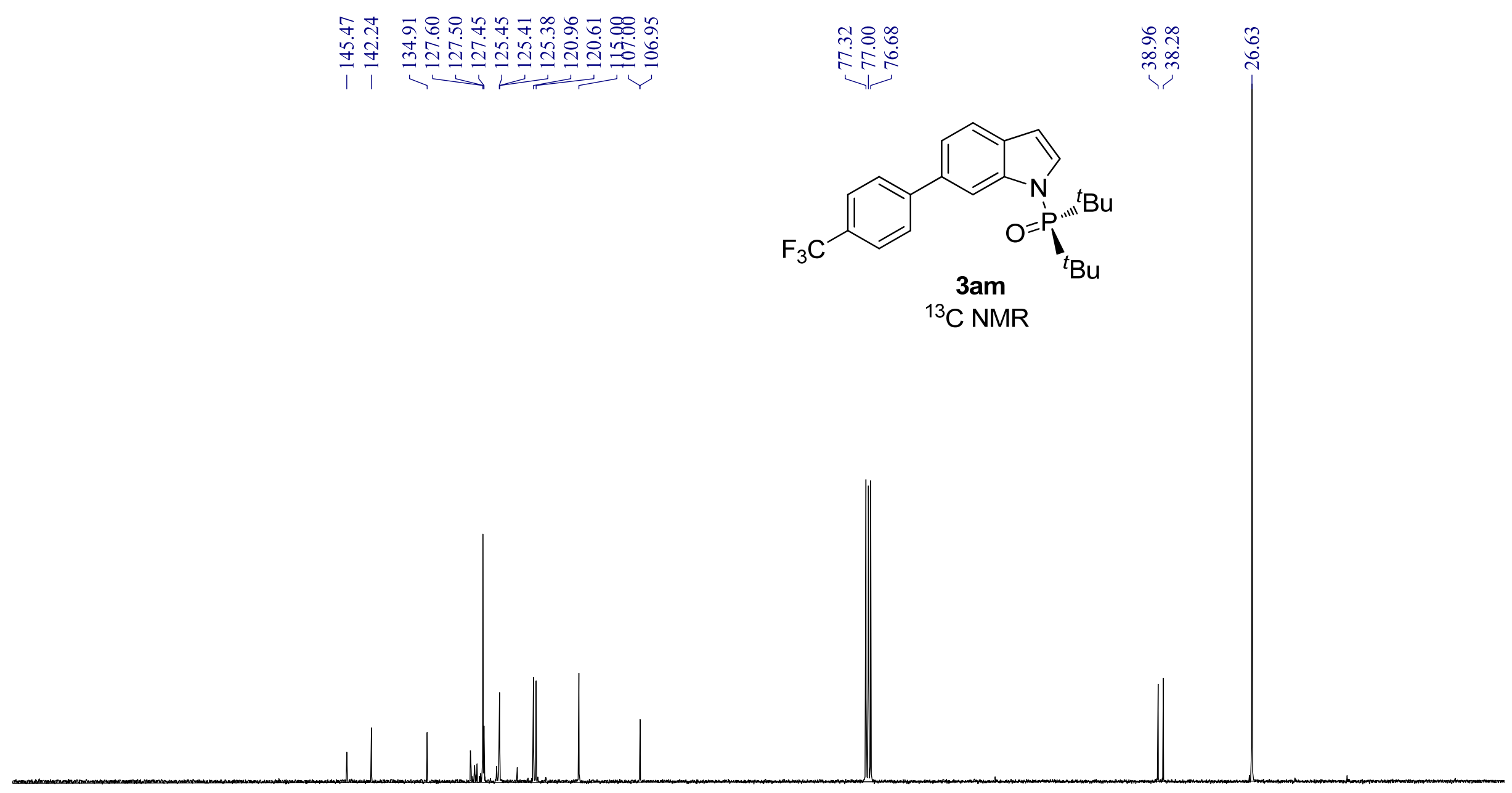

$\begin{array}{llllllllllllllllllllllllllllllllllllllllllllllllllllllll}185 & 180 & 175 & 170 & 165 & 160 & 155 & 150 & 145 & 140 & 135 & 130 & 125 & 120 & 115 & 110 & 105 & 100 & 95 & 90 & 85 & 80 & 75 & 70 & 65 & 60 & 55 & 50 & 45 & 40 & 35 & 30 & 25 & 20 & 15 & 10 & 5 & 0 & -5\end{array}$ 
$\overrightarrow{6}$
$\ddot{6}$

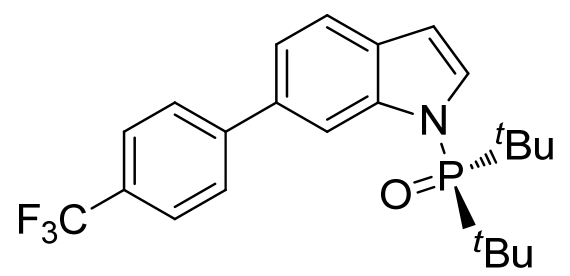

3am

${ }^{31} \mathrm{P}$ NMR

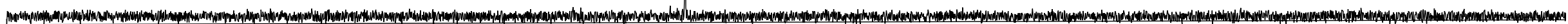

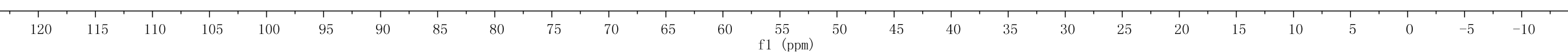



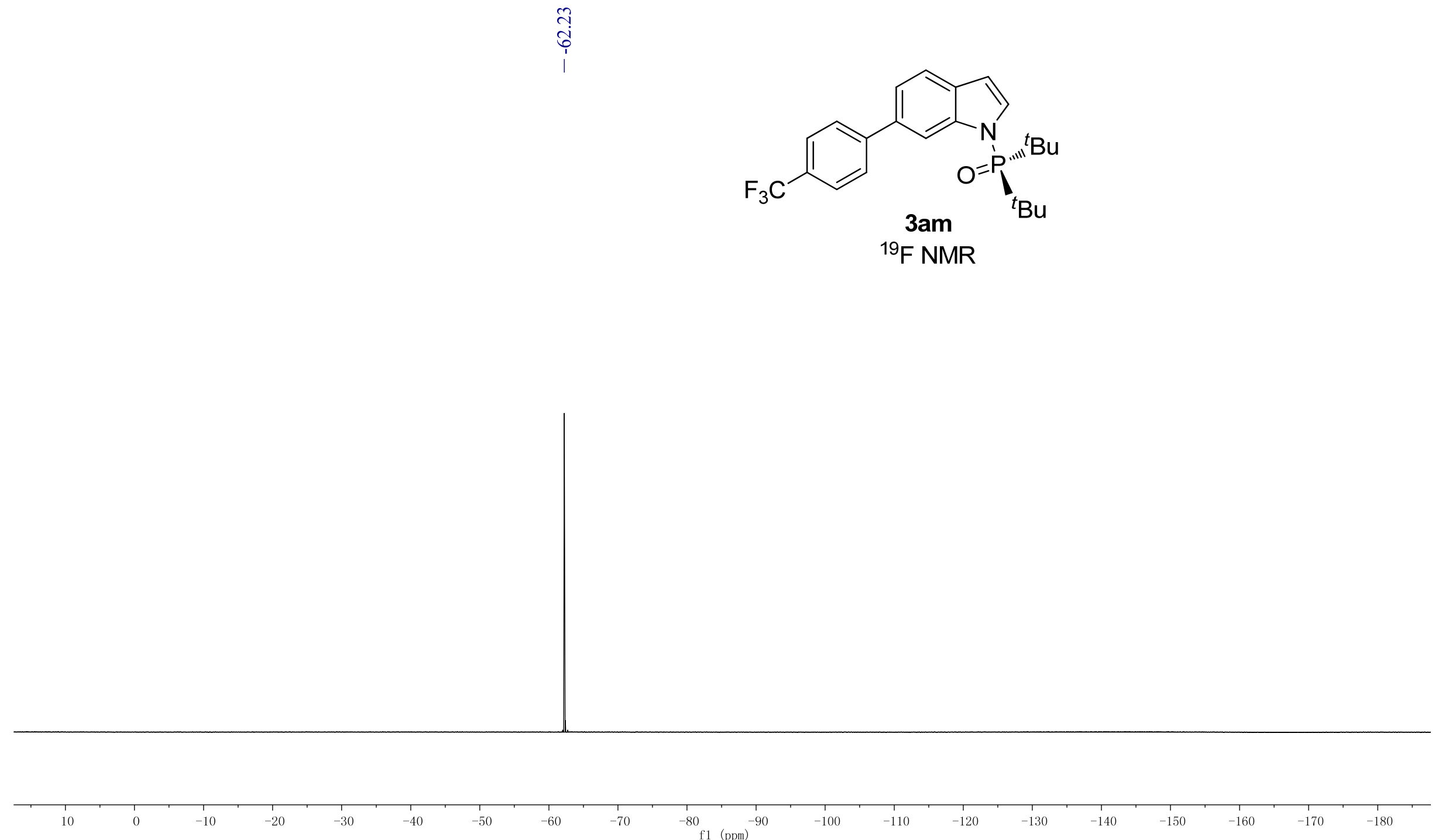
$\infty$
$\infty$
$\infty$

i.

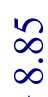

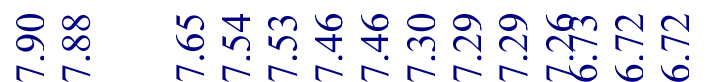

i,

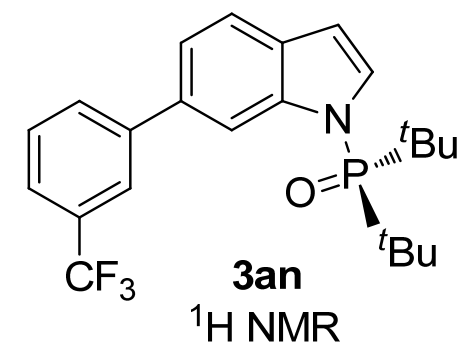

กิต
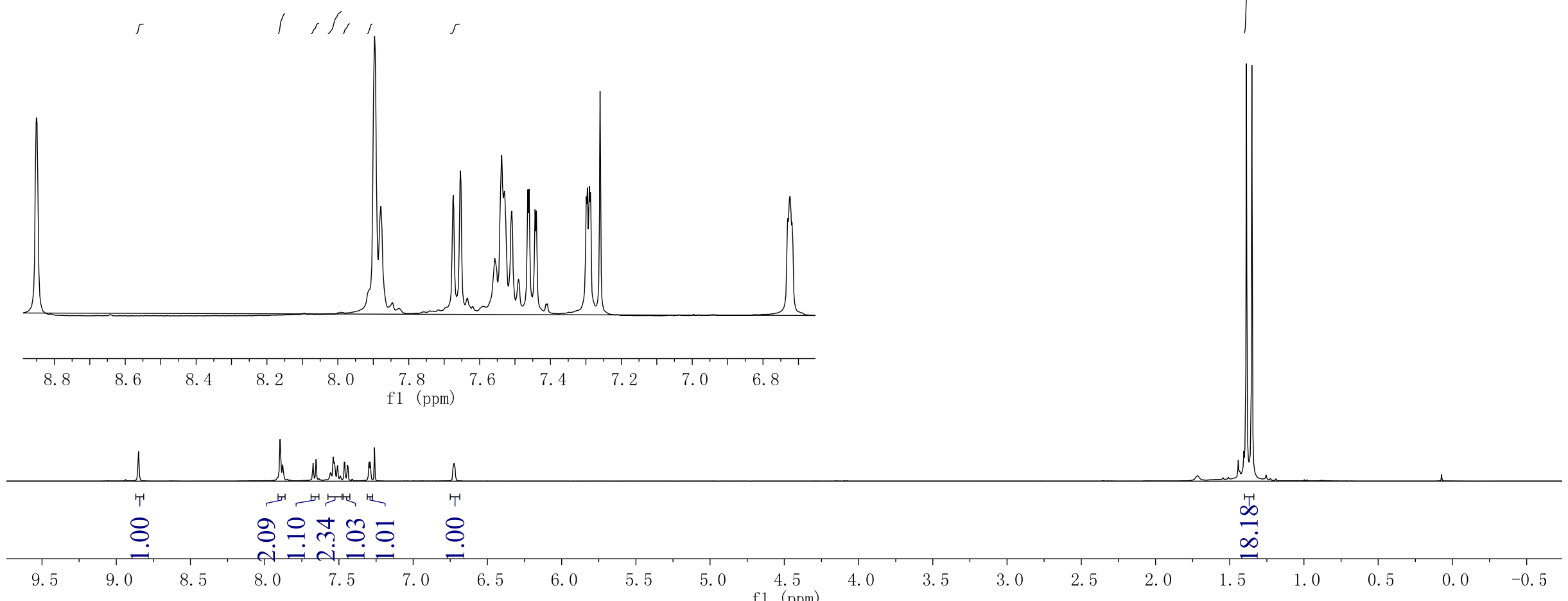


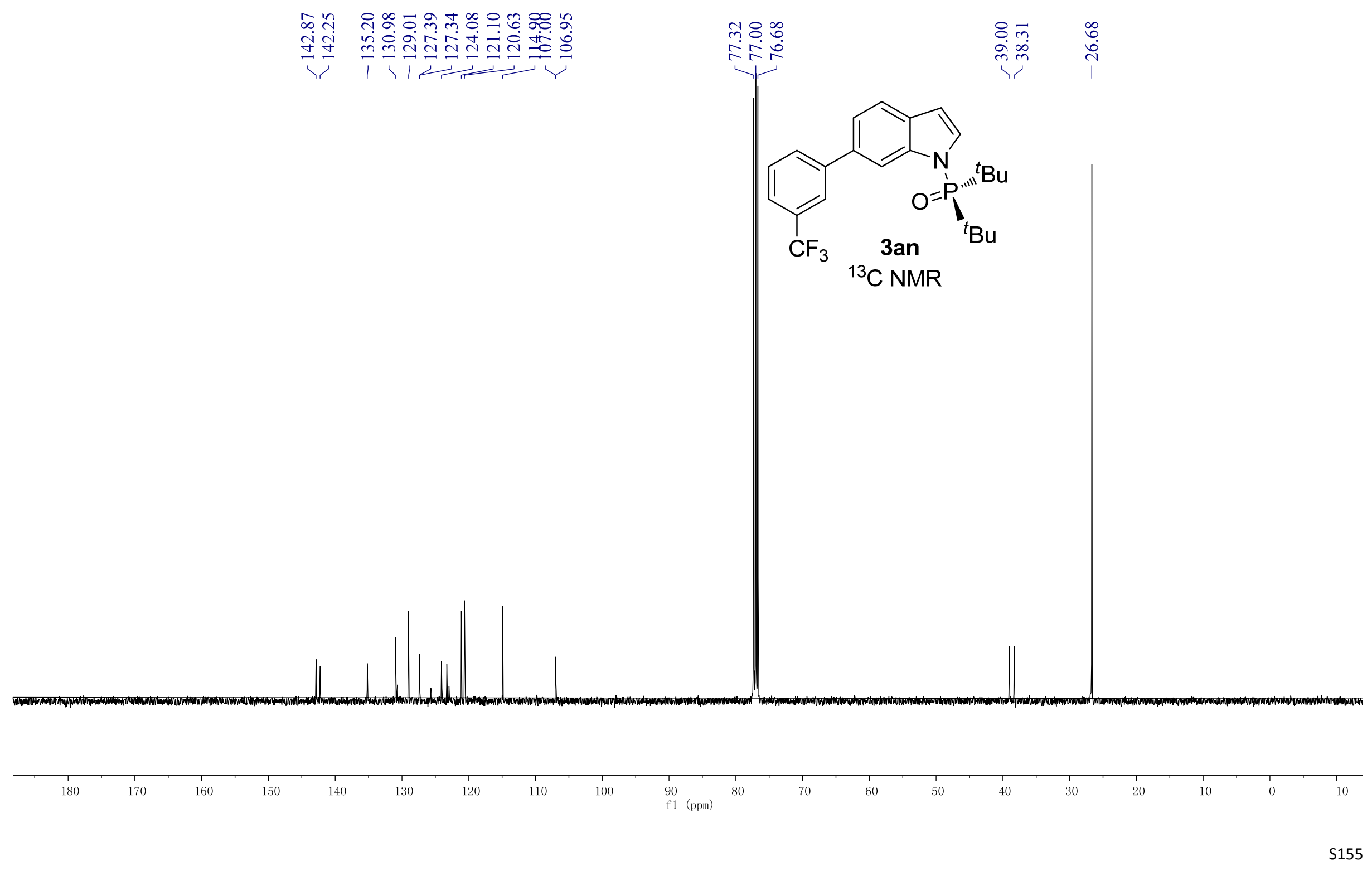



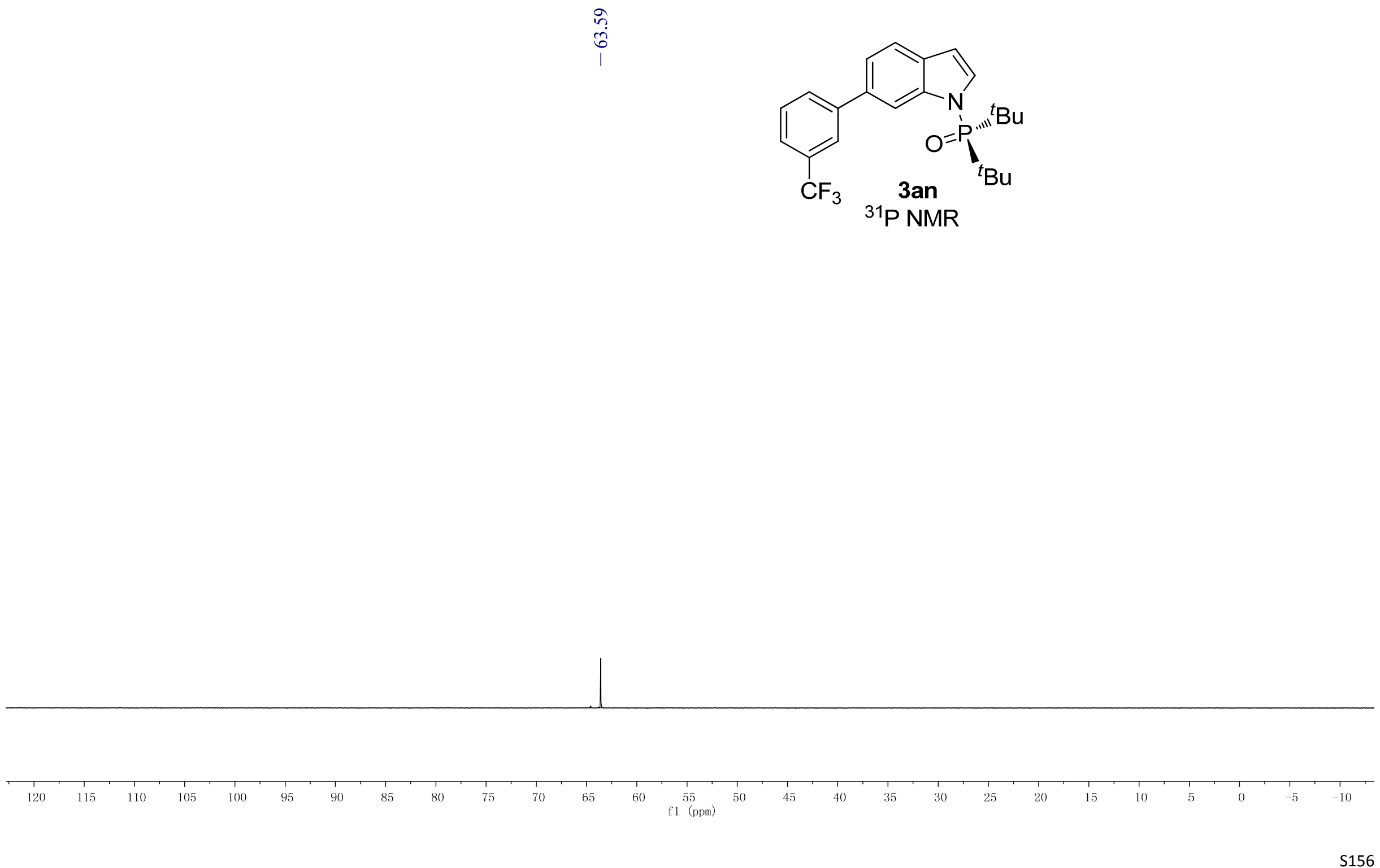

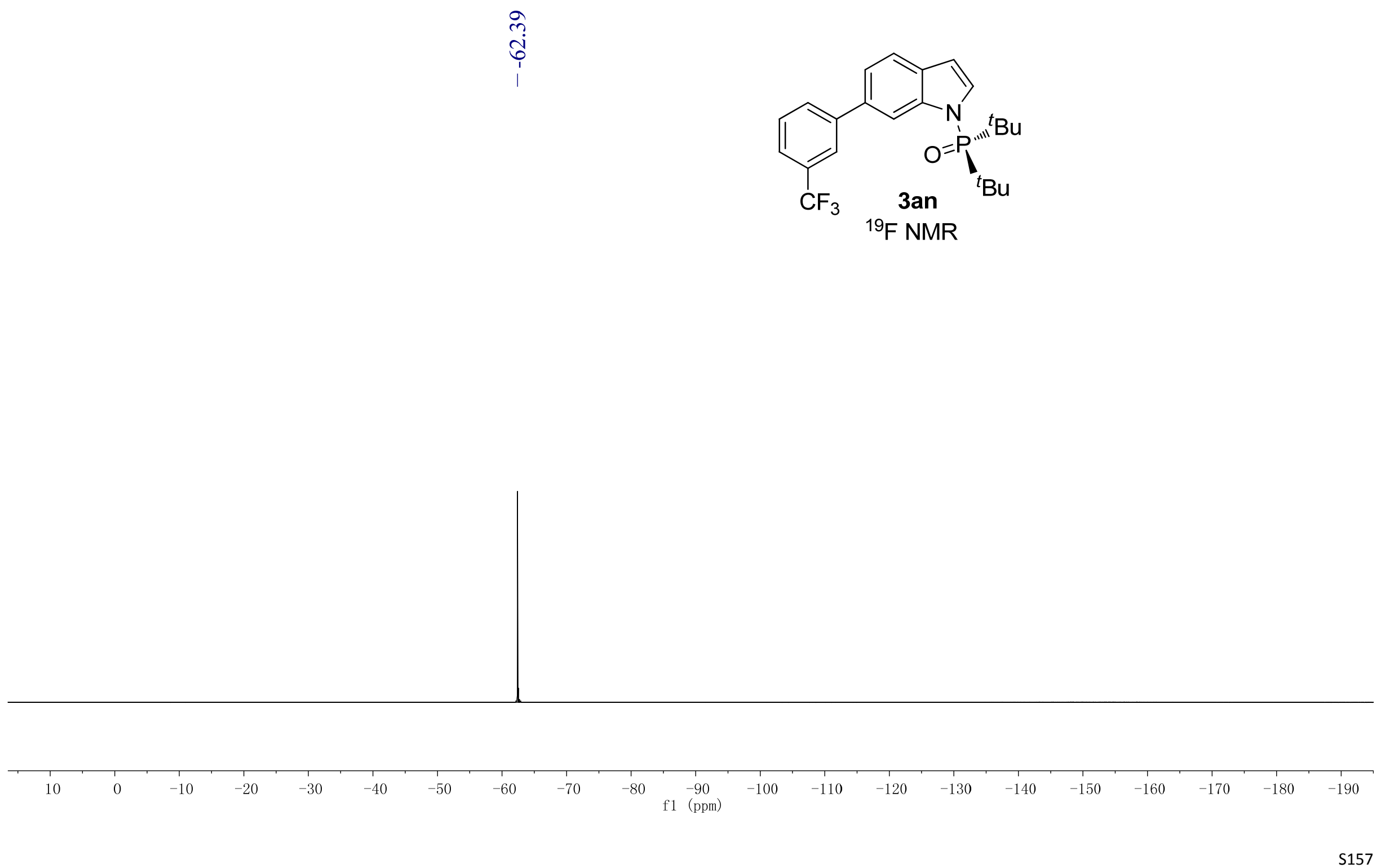


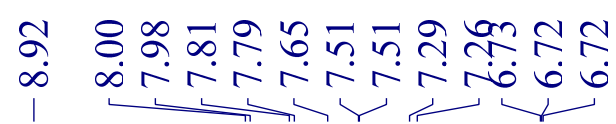

तु

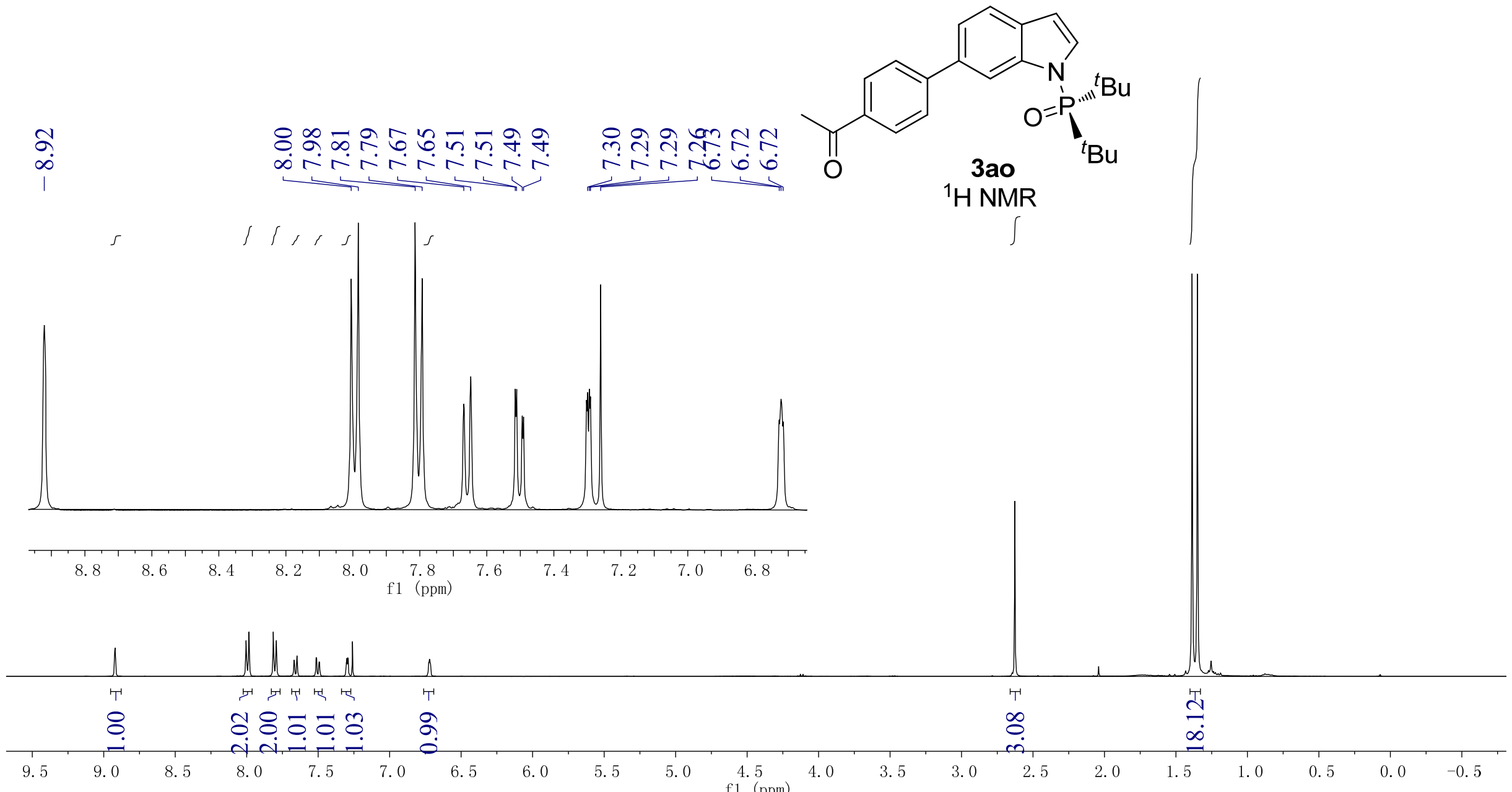




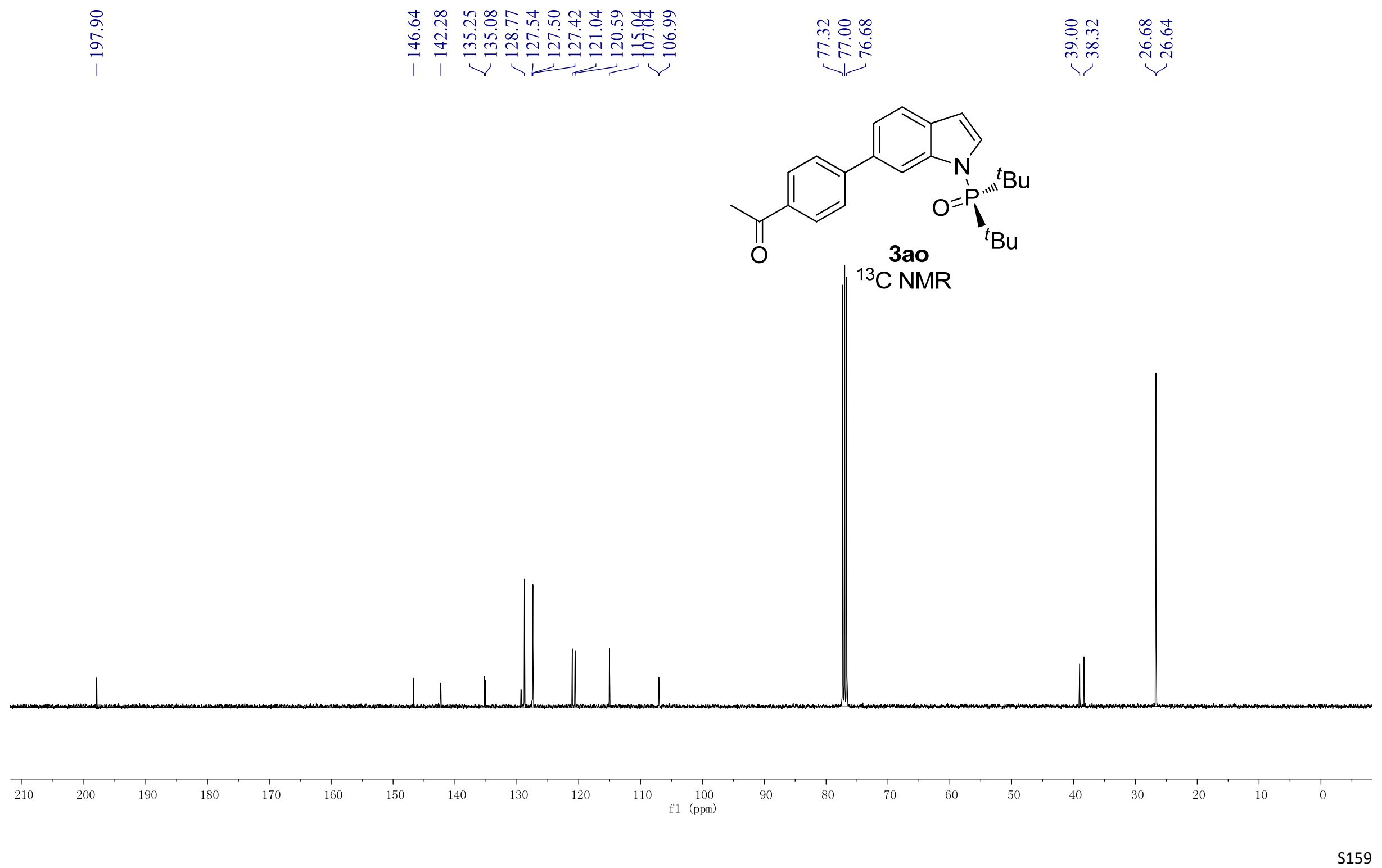


8
0
0
1
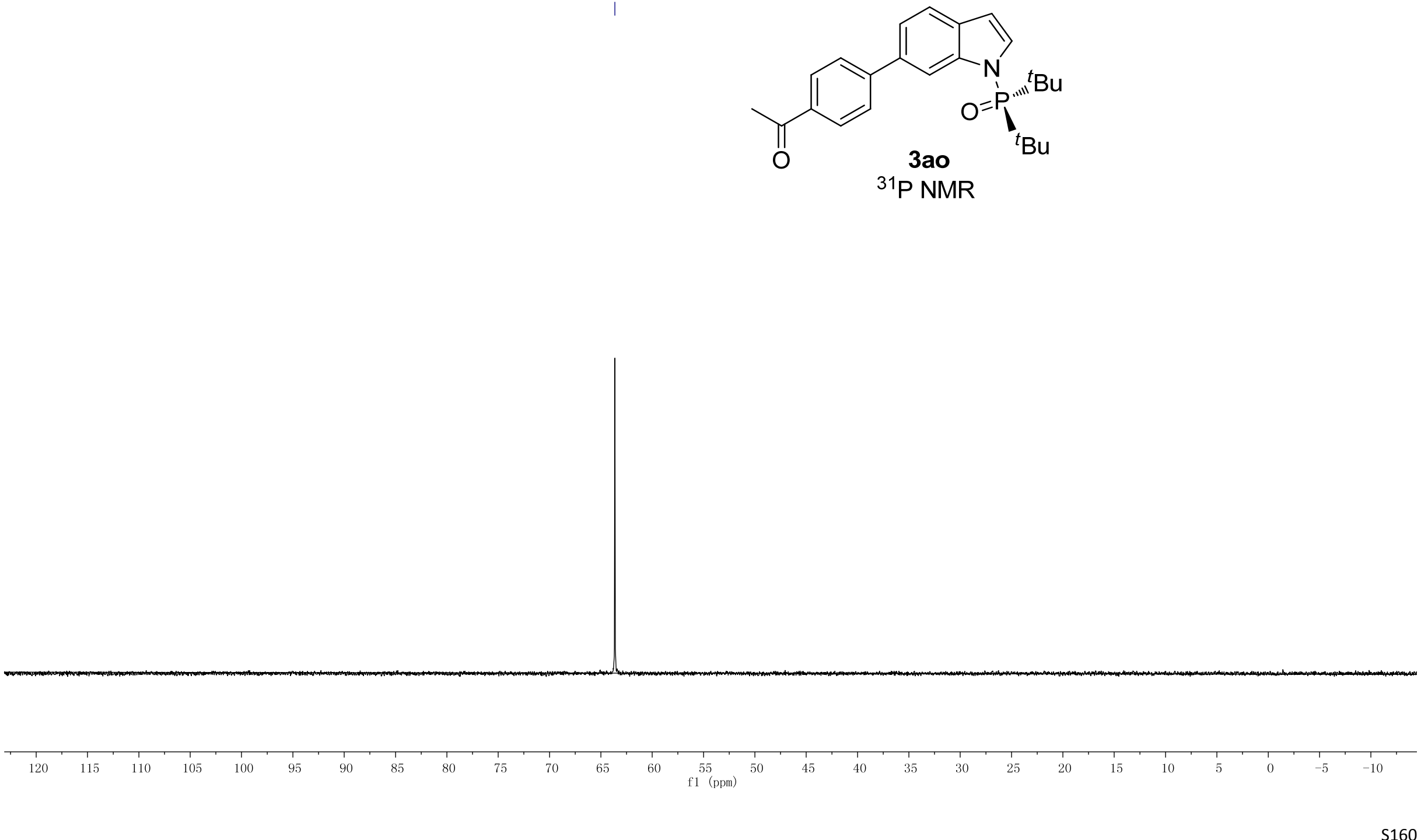


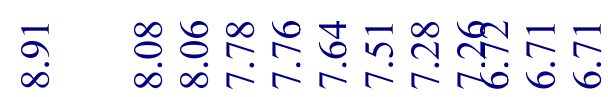

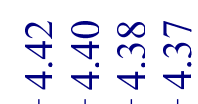

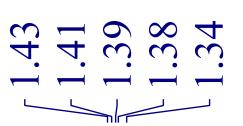

$r=1, r, j$
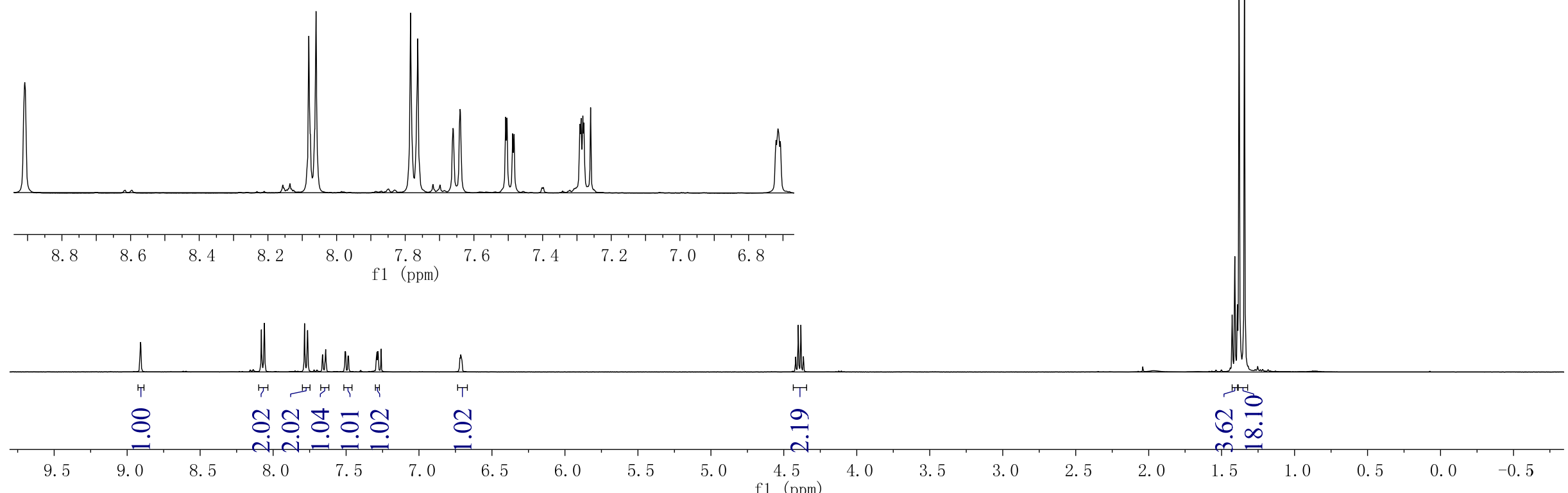


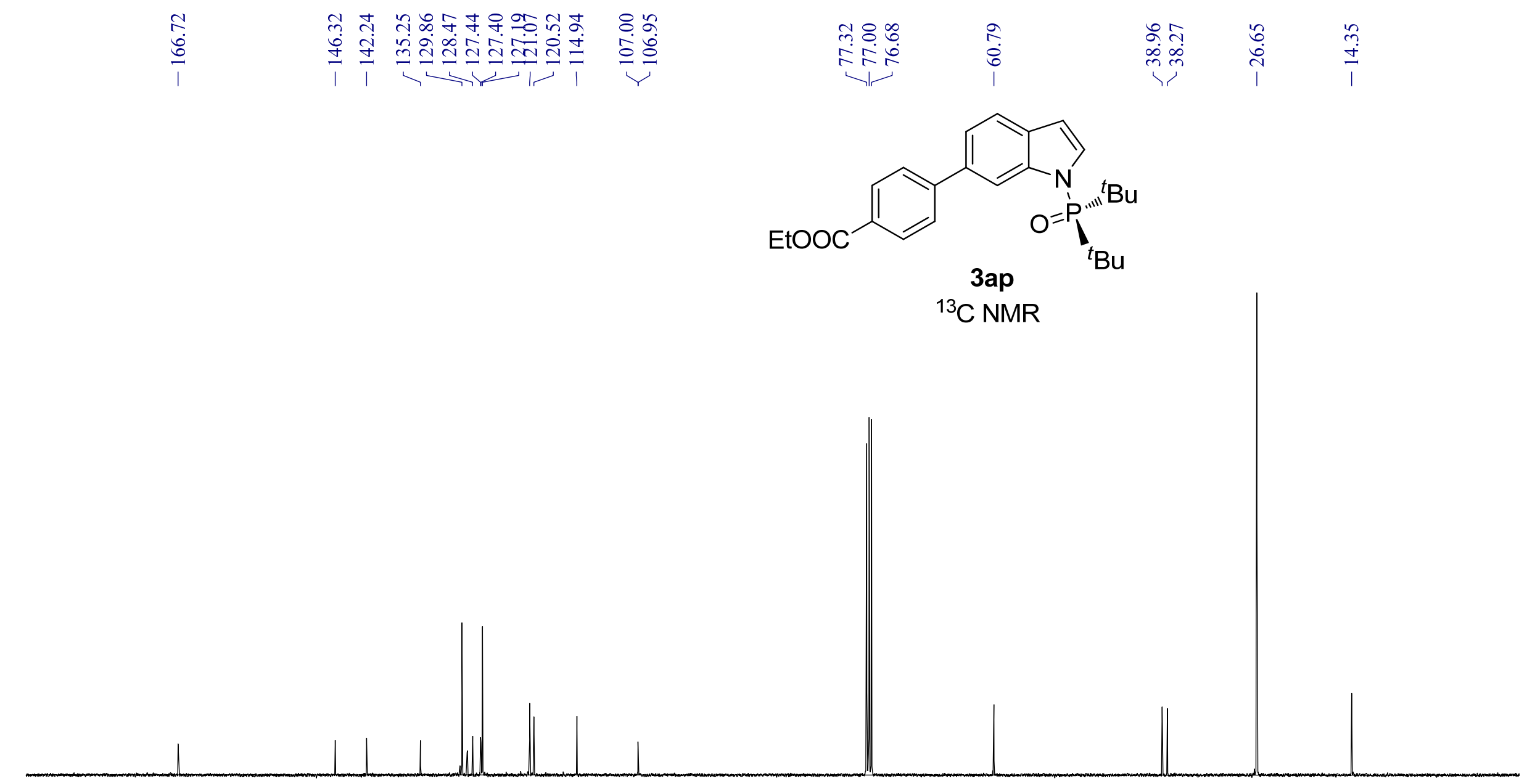

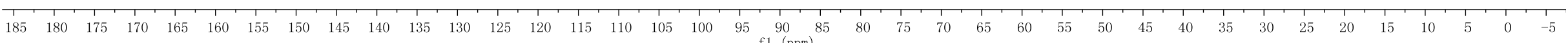




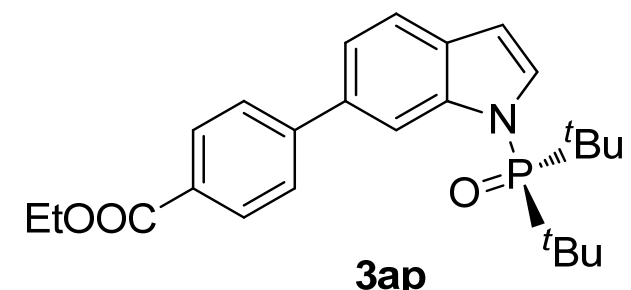

${ }^{31} \mathrm{P}$ NMR

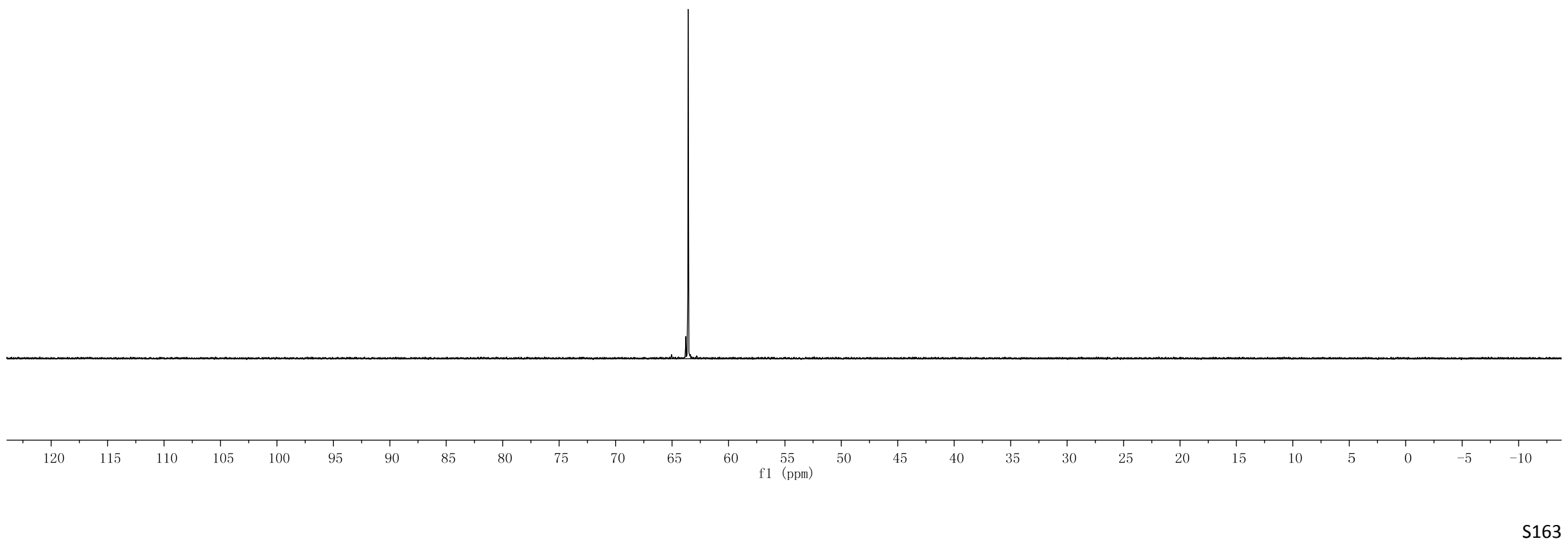




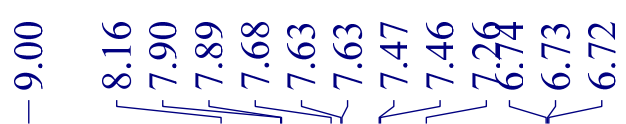

웅

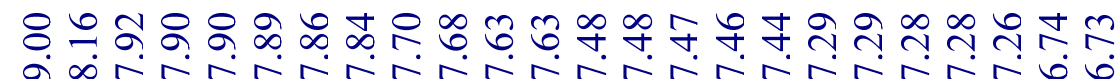

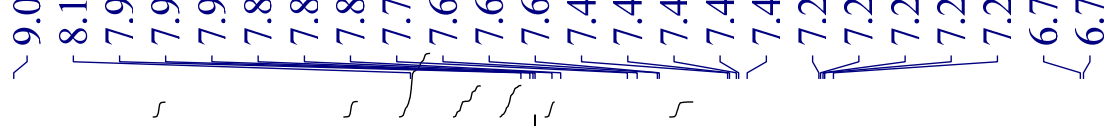
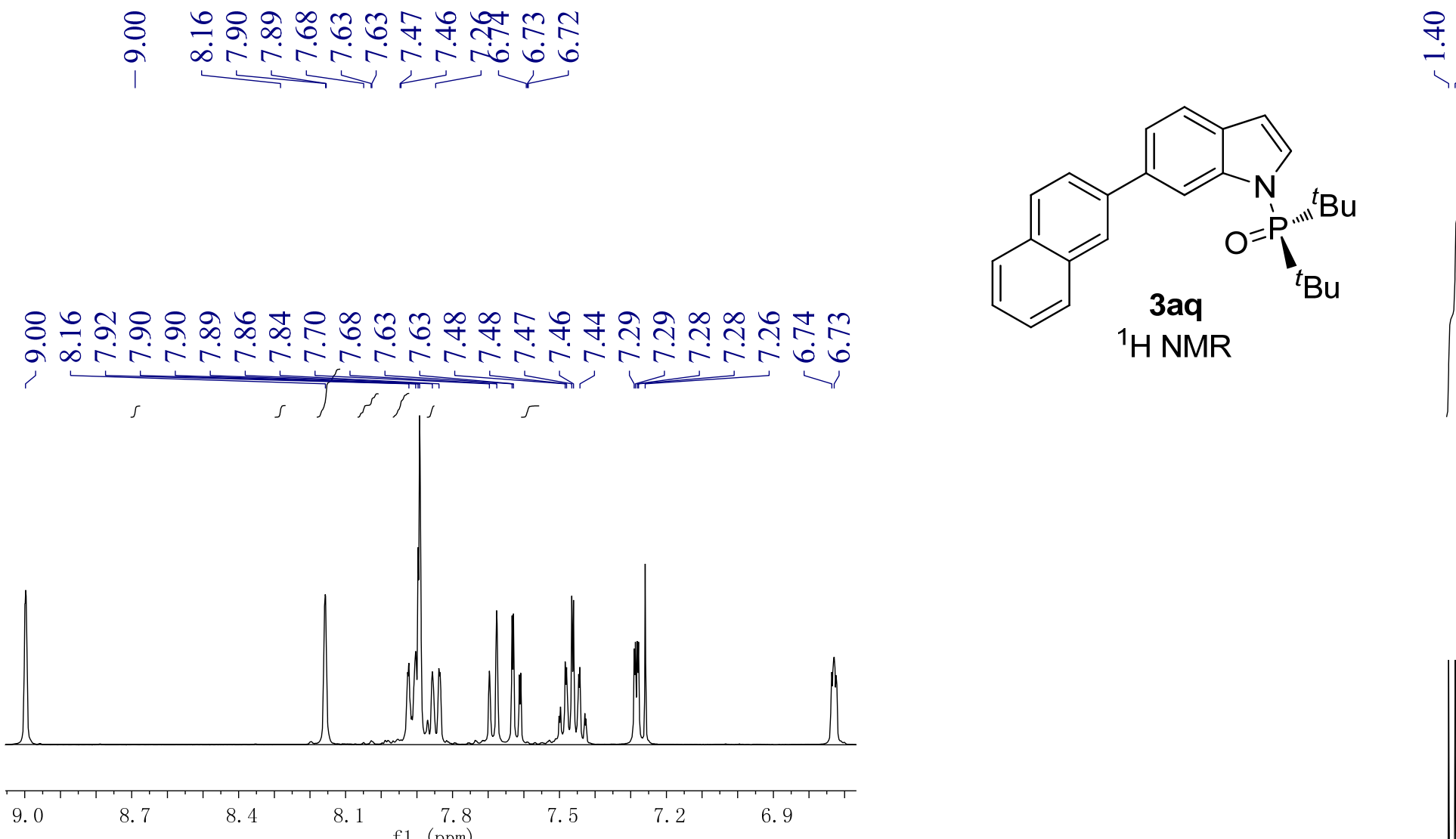

f1 (ppm)
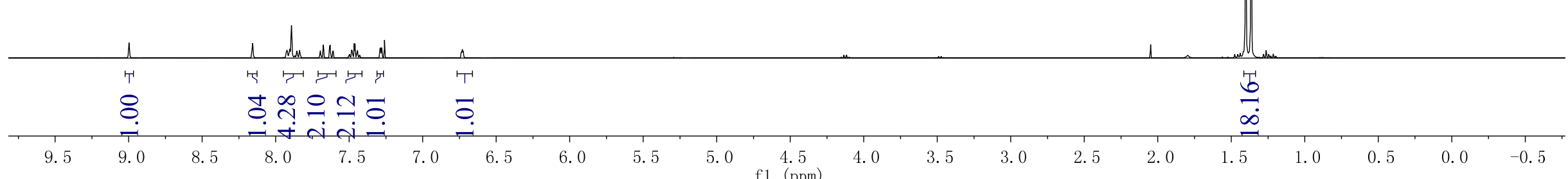


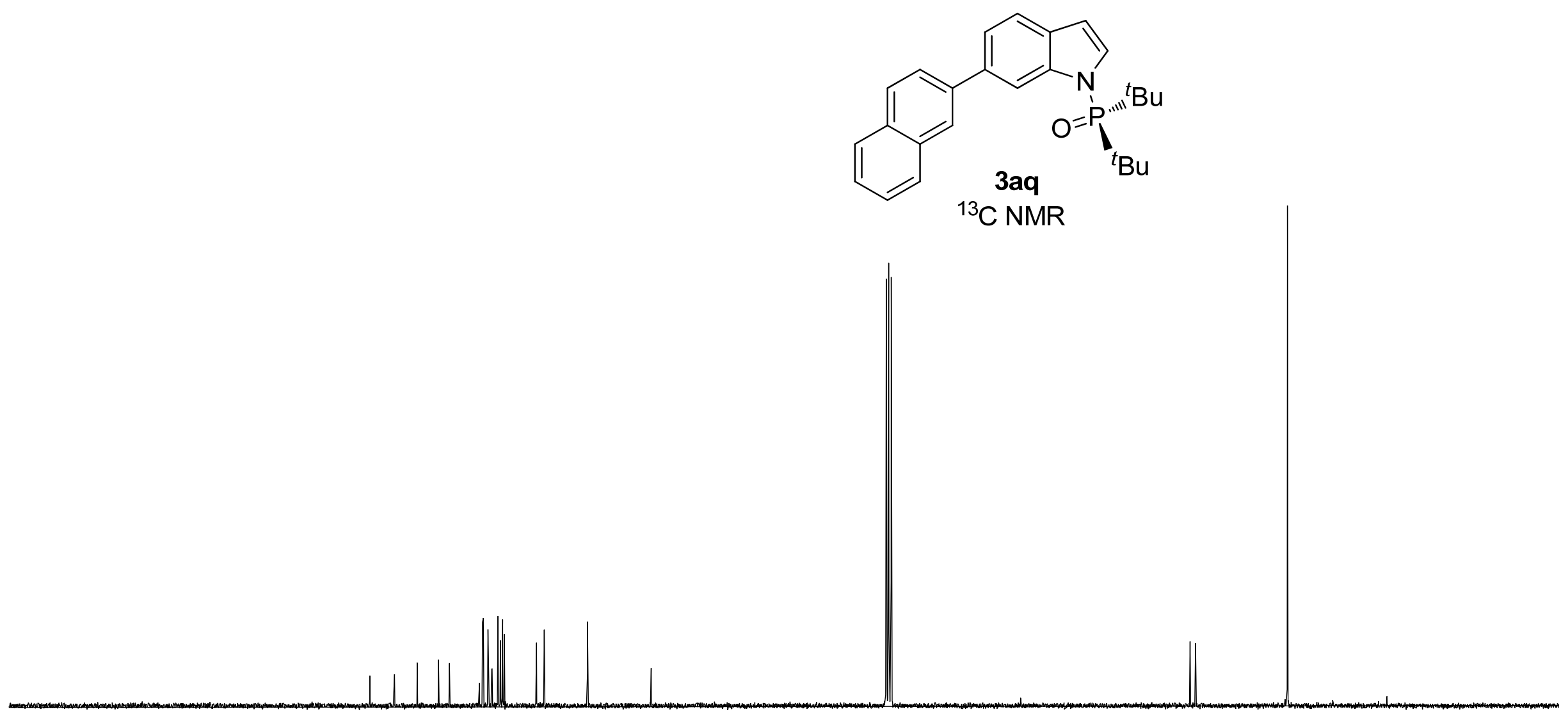

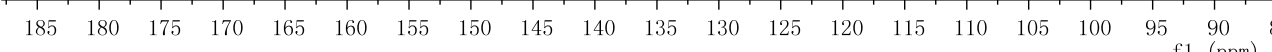



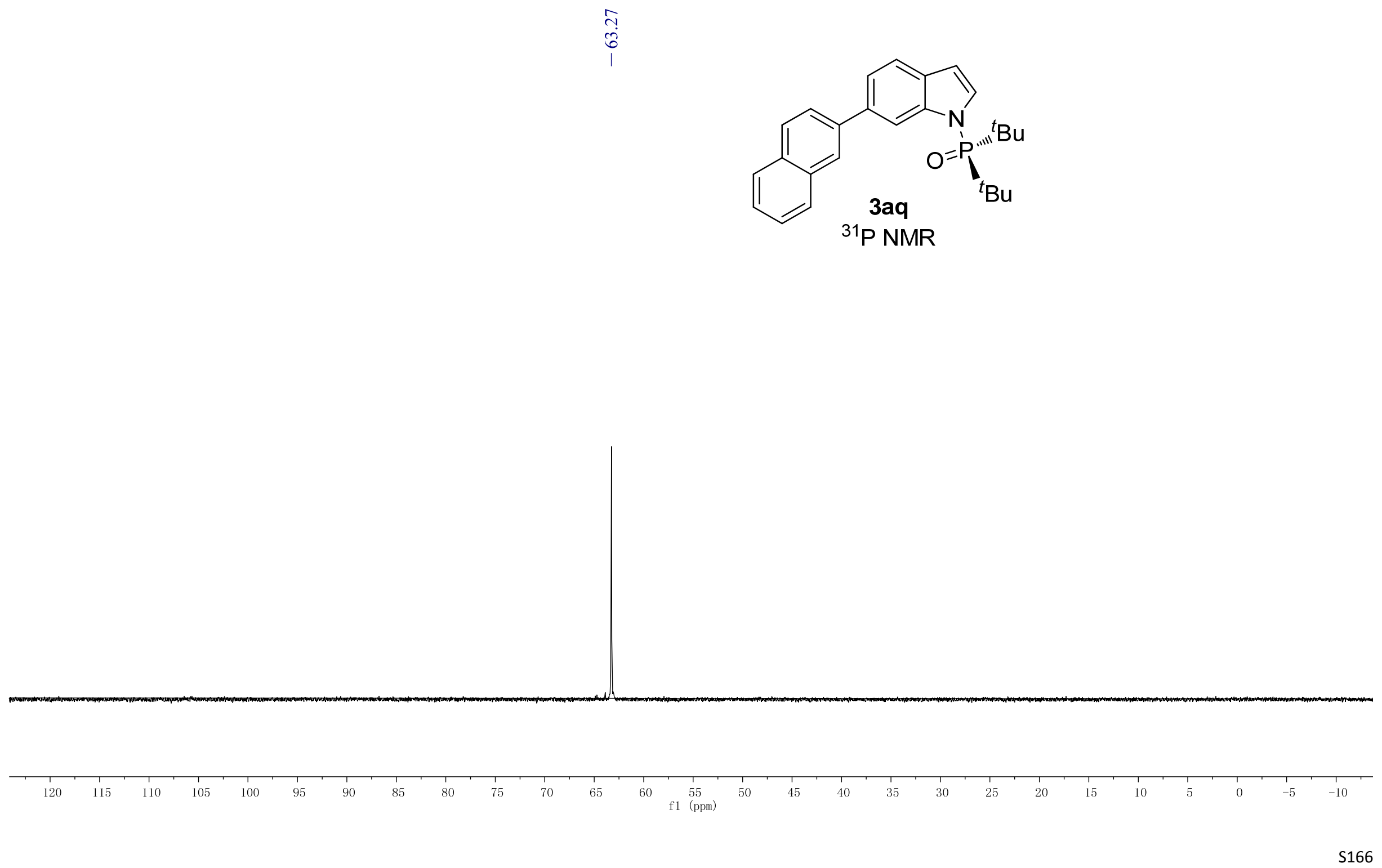


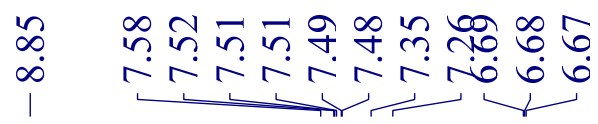

국

$n$
$\infty$
$\infty$

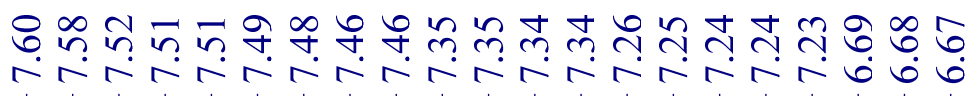

$, 1,1$
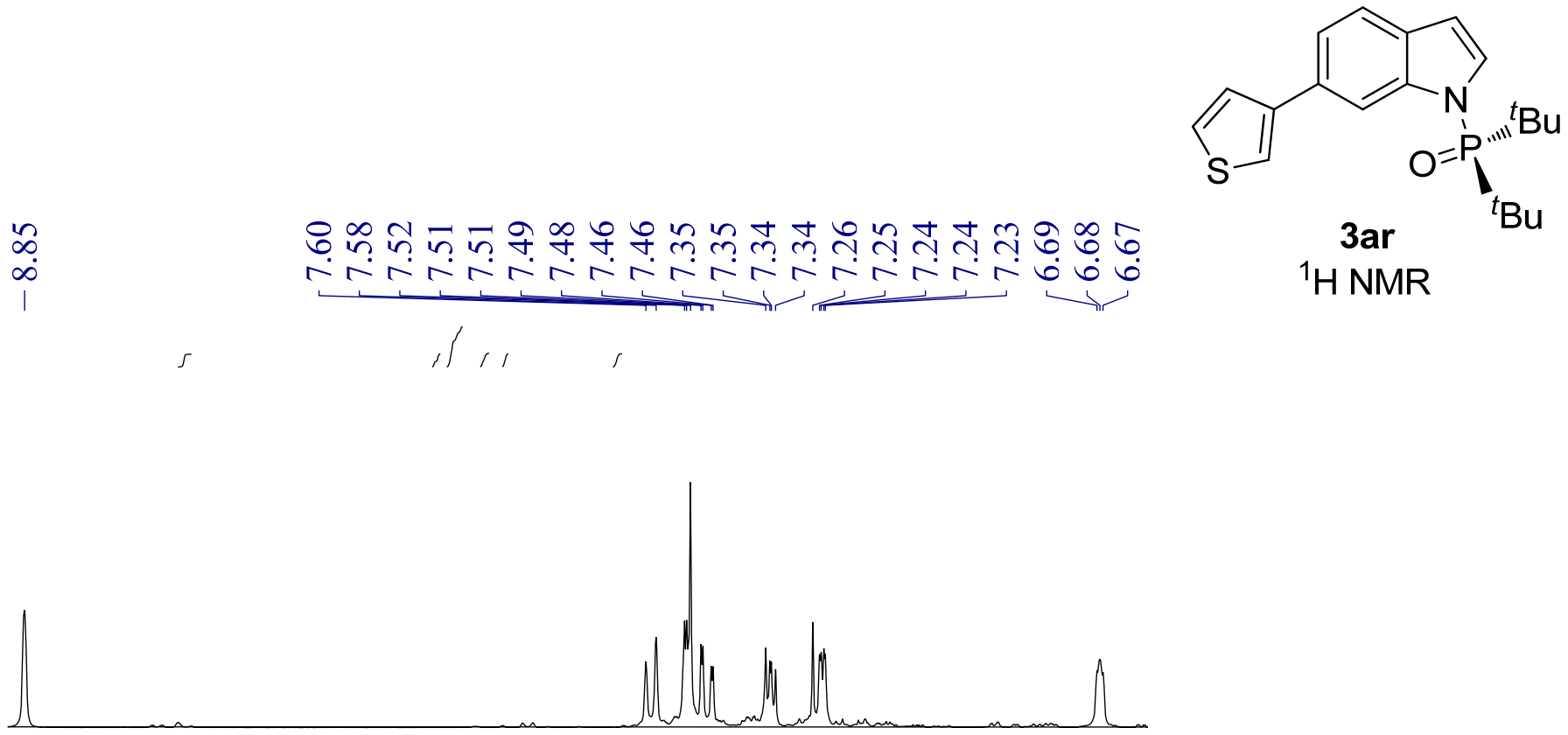

${ }^{1} \mathrm{H}$ NMR
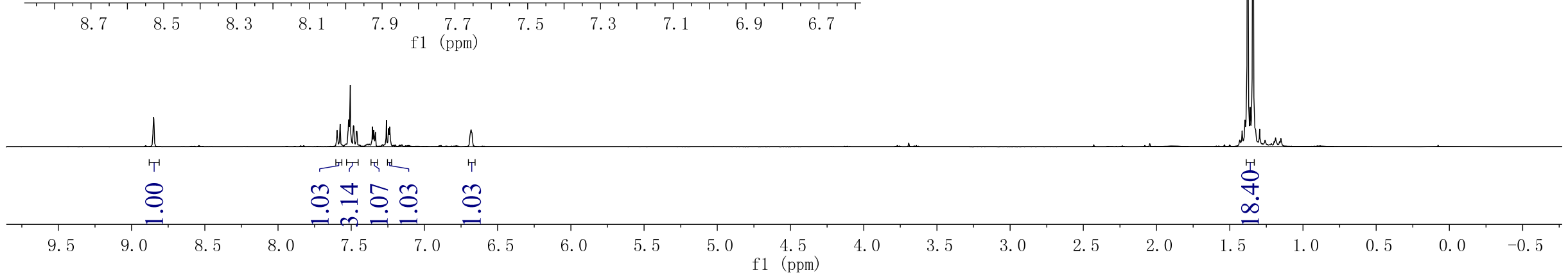


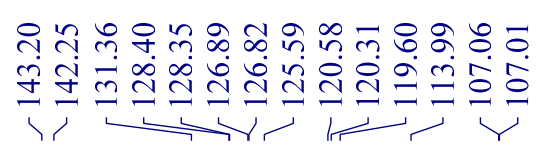

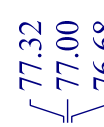

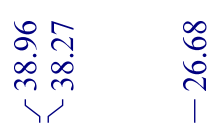

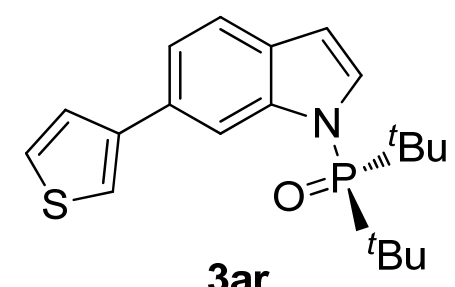

${ }^{13} \mathrm{C}$ NMR

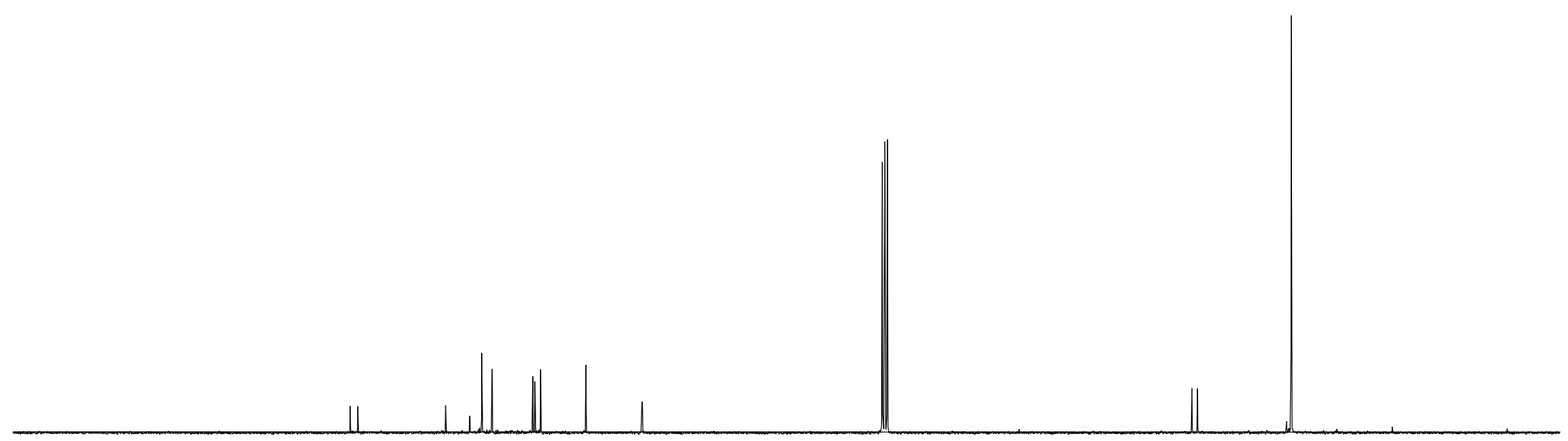

$\begin{array}{lllllllllllllllllllll}180 & 175 & 170 & 165 & 160 & 155 & 150 & 145 & 140 & 135 & 130 & 125 & 120 & 115 & 110 & 105 & 100 & 95 & 90 & 8\end{array}$ 

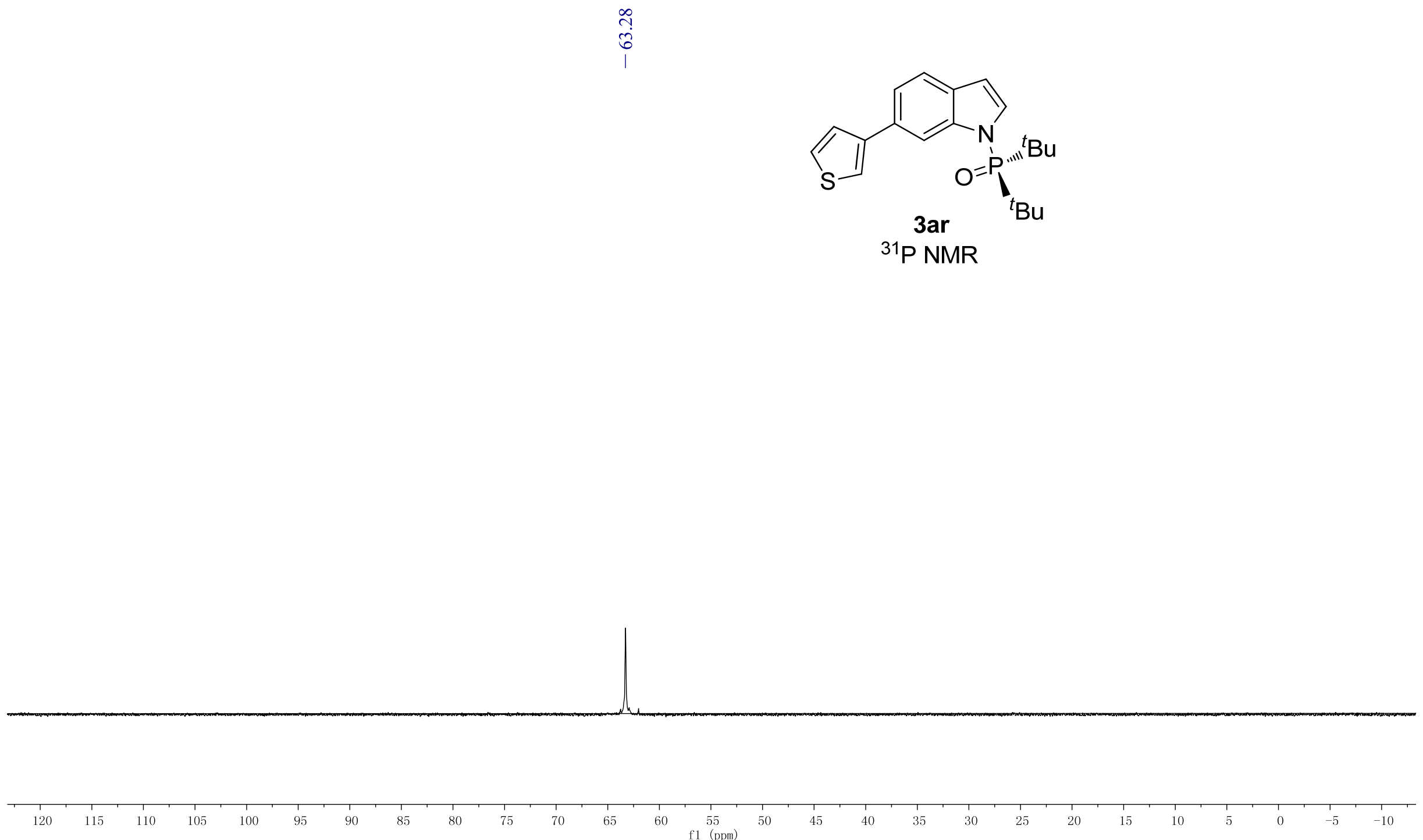

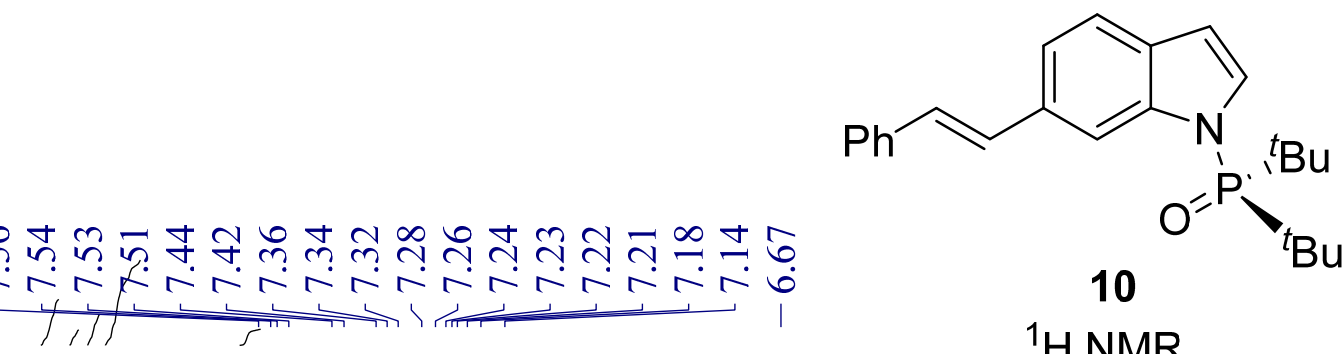

${ }^{1} \mathrm{H}$ NMR
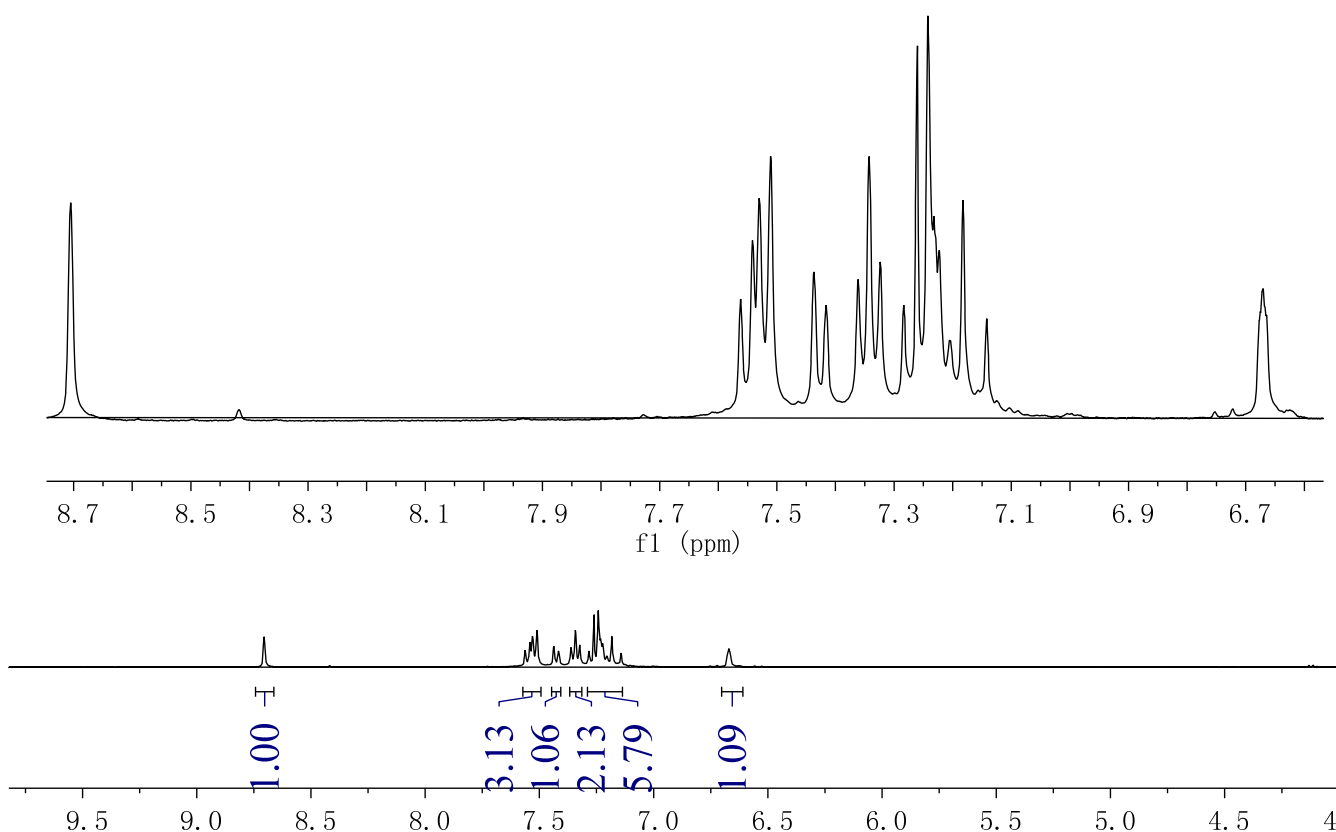

Munull मिस्समत m

9.59 .0 ल तो 


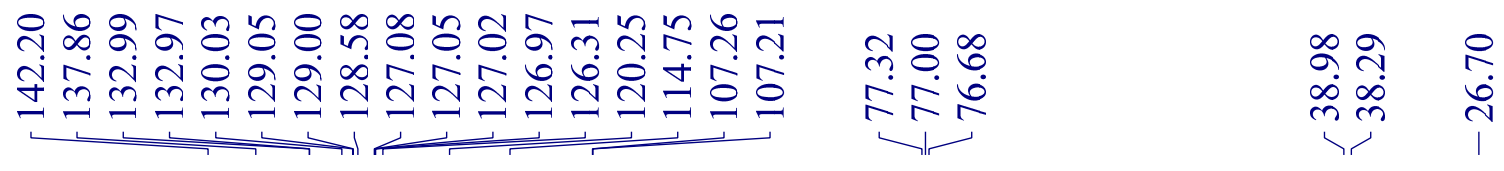

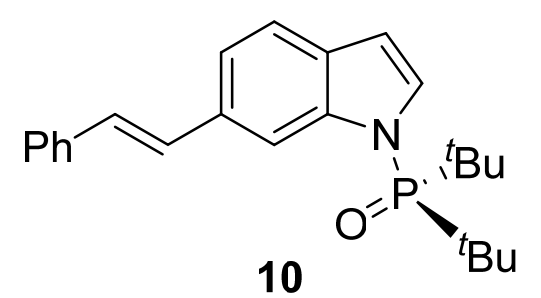

${ }^{13} \mathrm{C}$ NMR

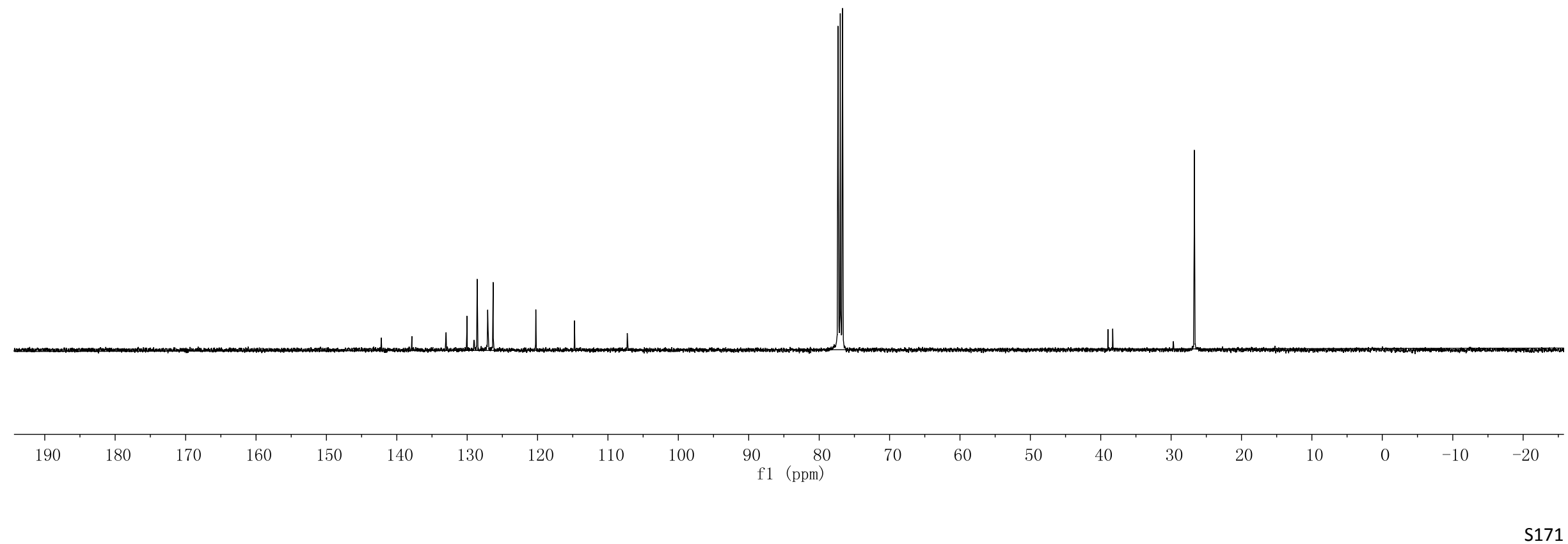


ते

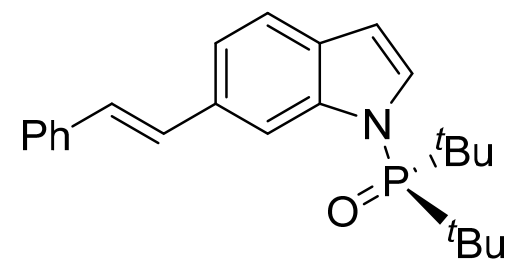

10
${ }^{31} \mathrm{P}$ NMR

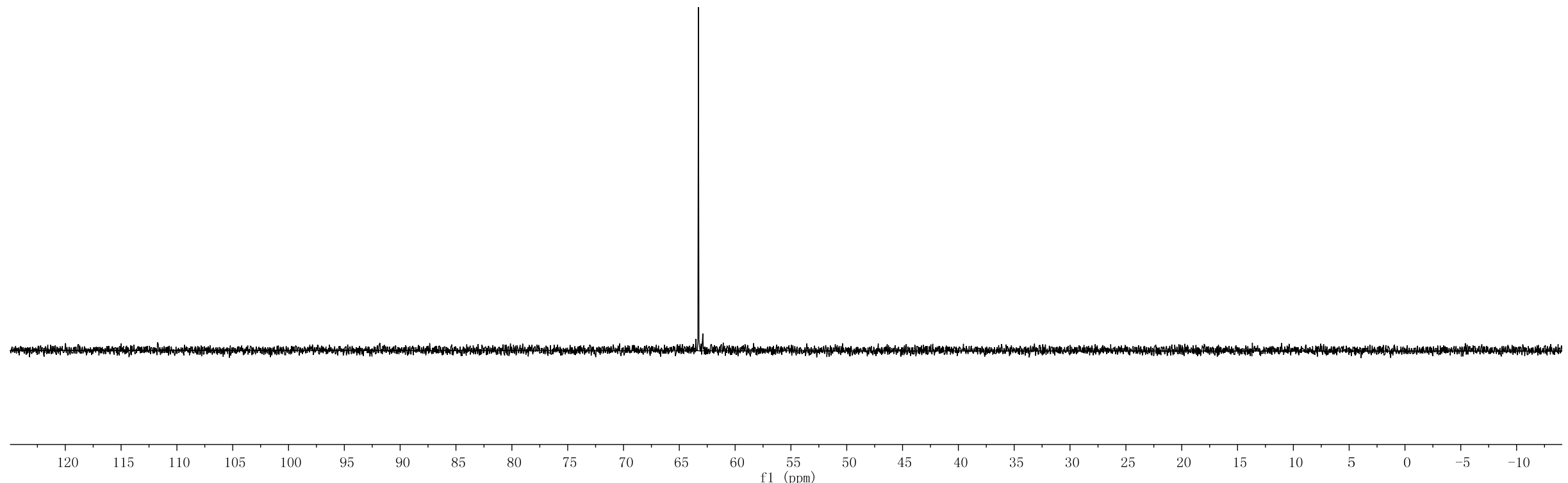


근

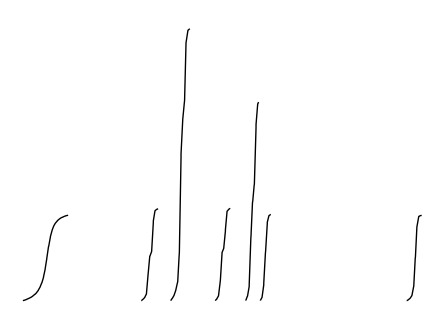

$\stackrel{?}{i}$

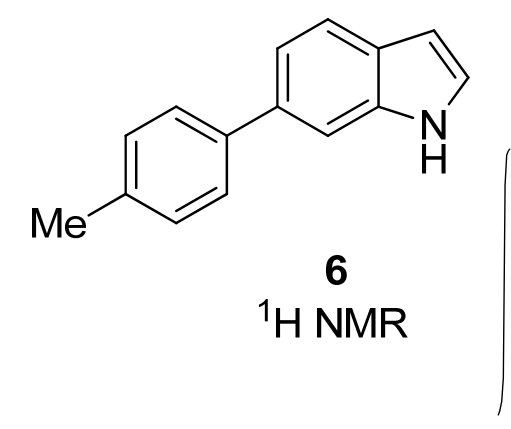

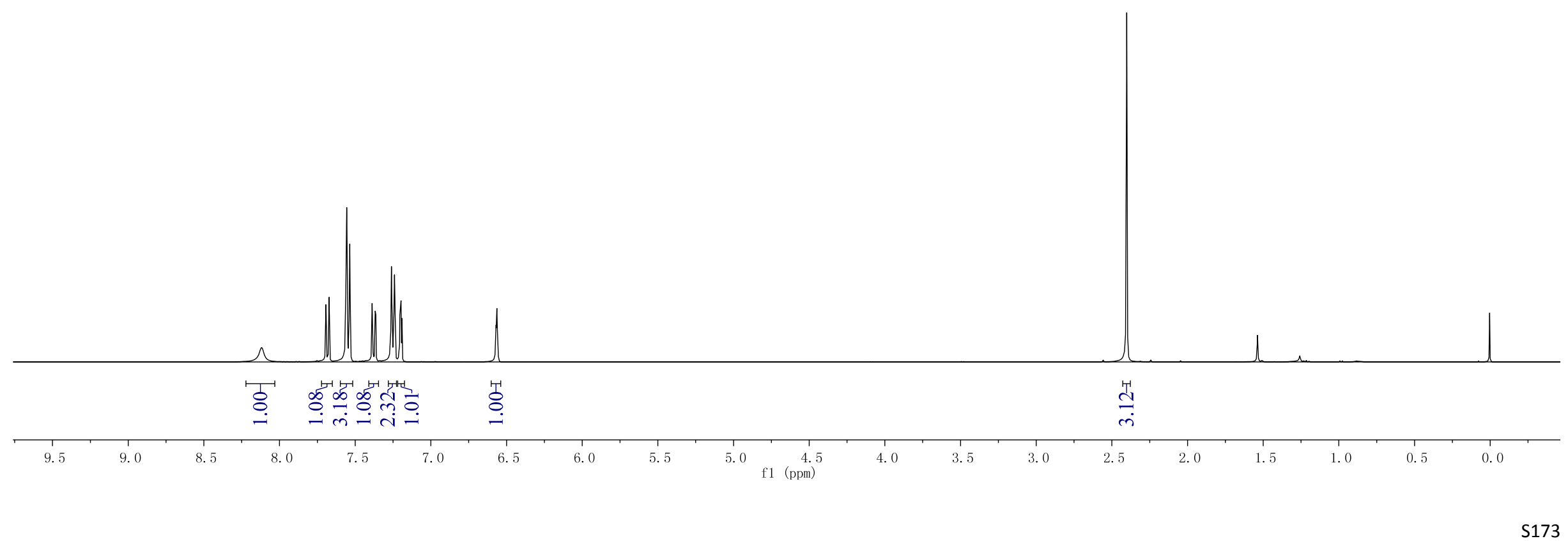




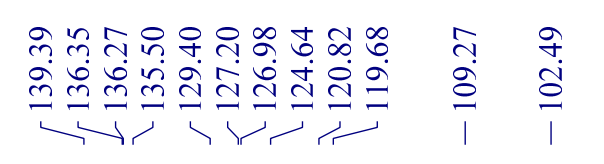

$$
\text { 옹ํㅇ }
$$

$\frac{\circ}{i}$

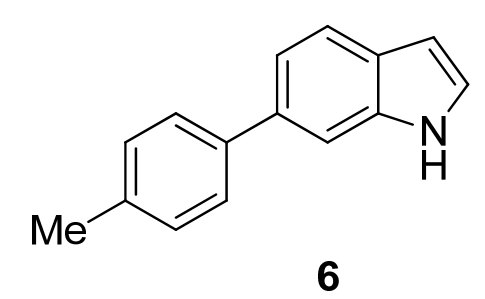

${ }^{13} \mathrm{C}$ NMR

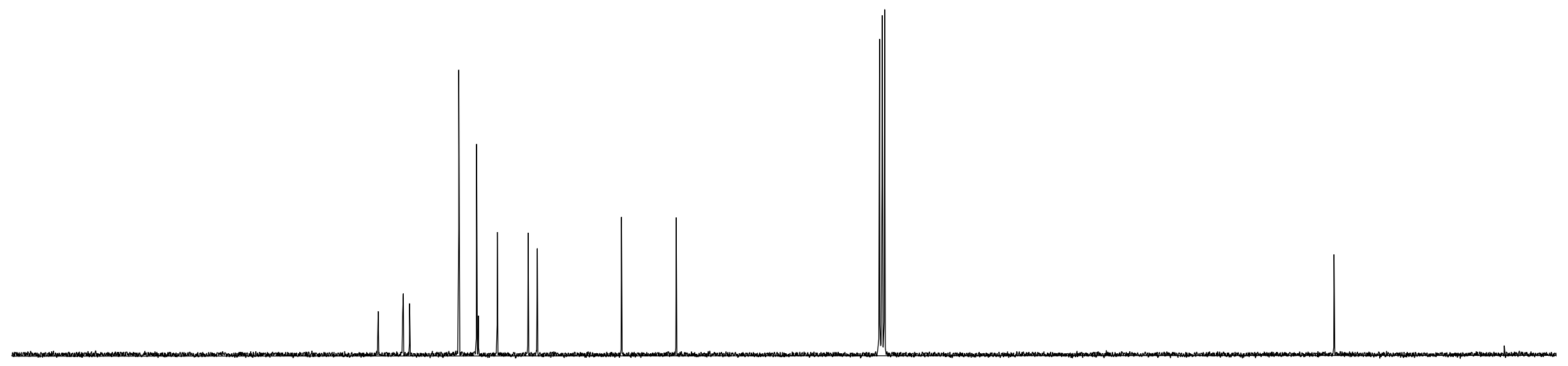

$\begin{array}{llllllllllllllllllllllll}180 & 175 & 170 & 165 & 160 & 155 & 150 & 145 & 140 & 135 & 130 & 125 & 120 & 115 & 110 & 105 & 100 & 95 & 90 & 85\end{array}$ 
m

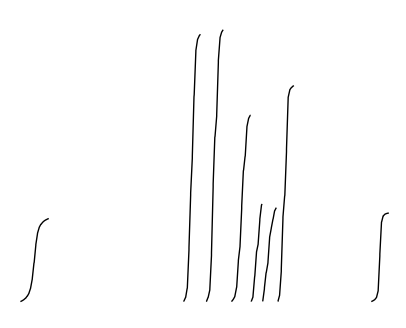

욱

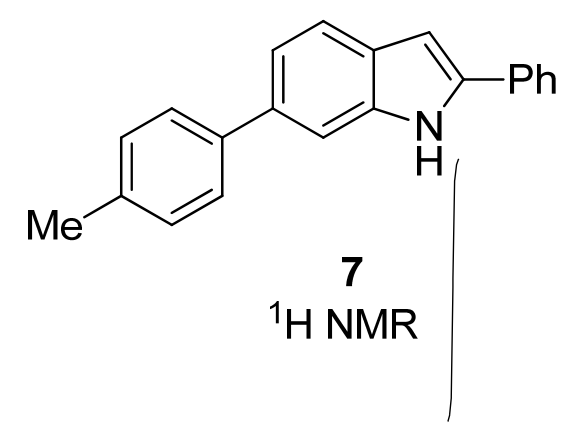

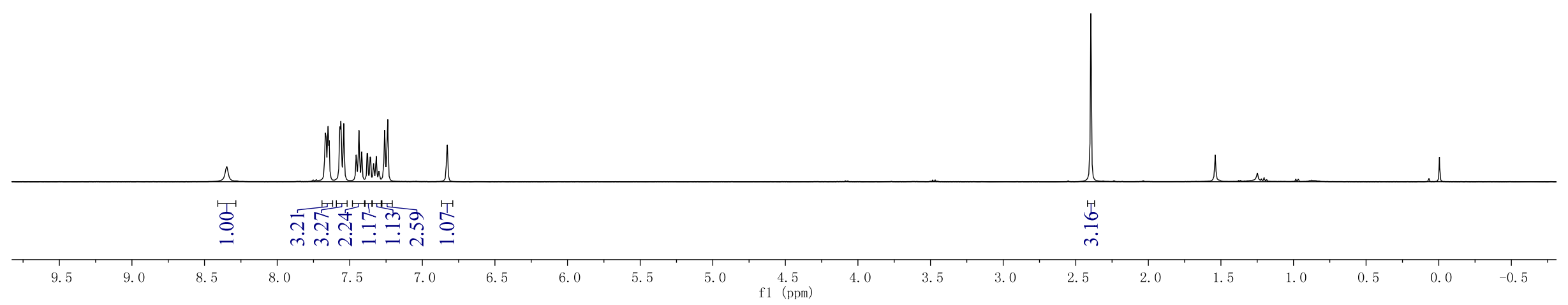




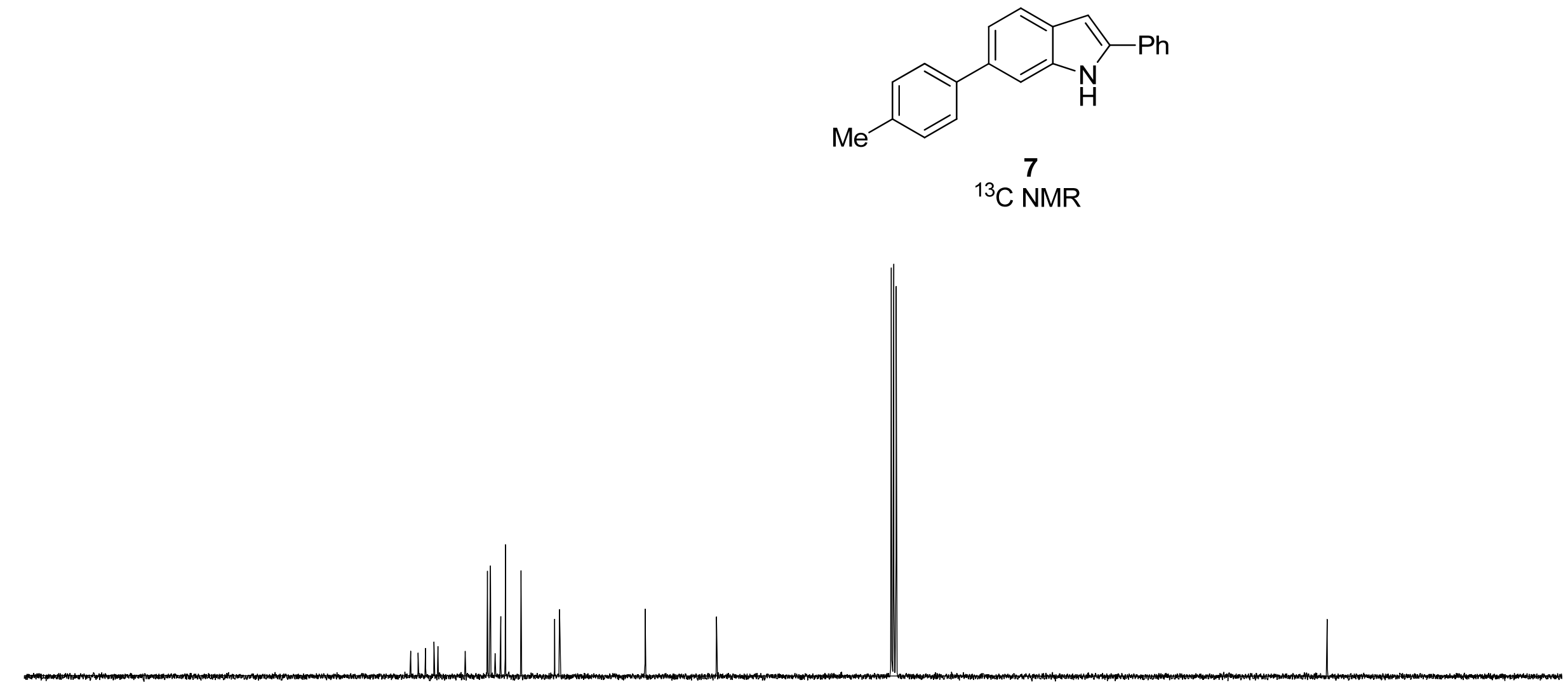

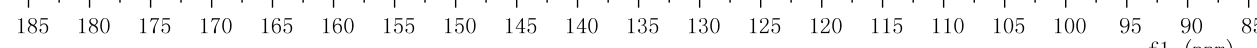




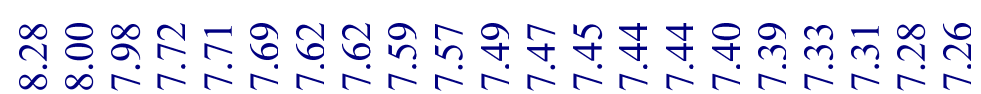
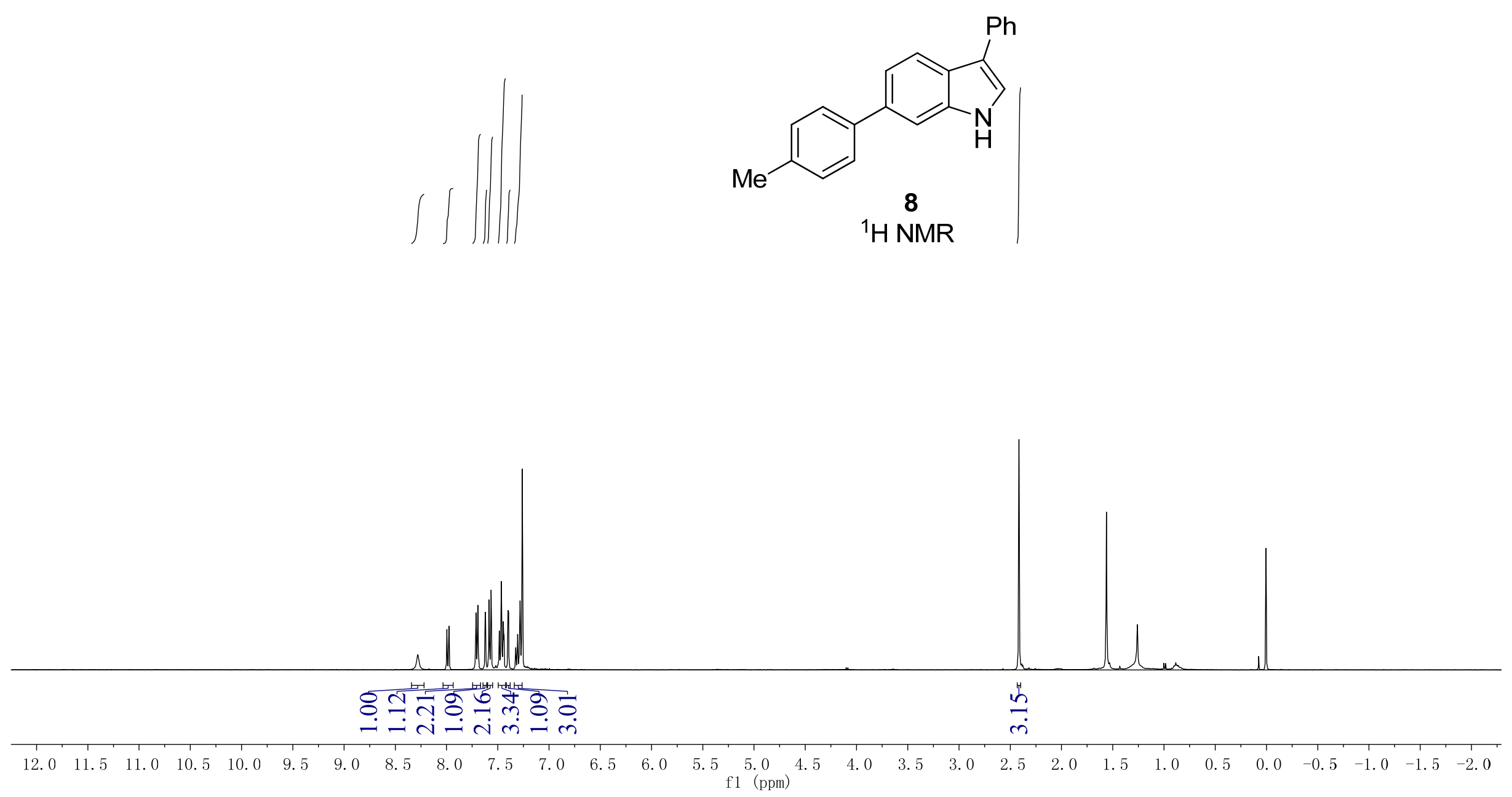


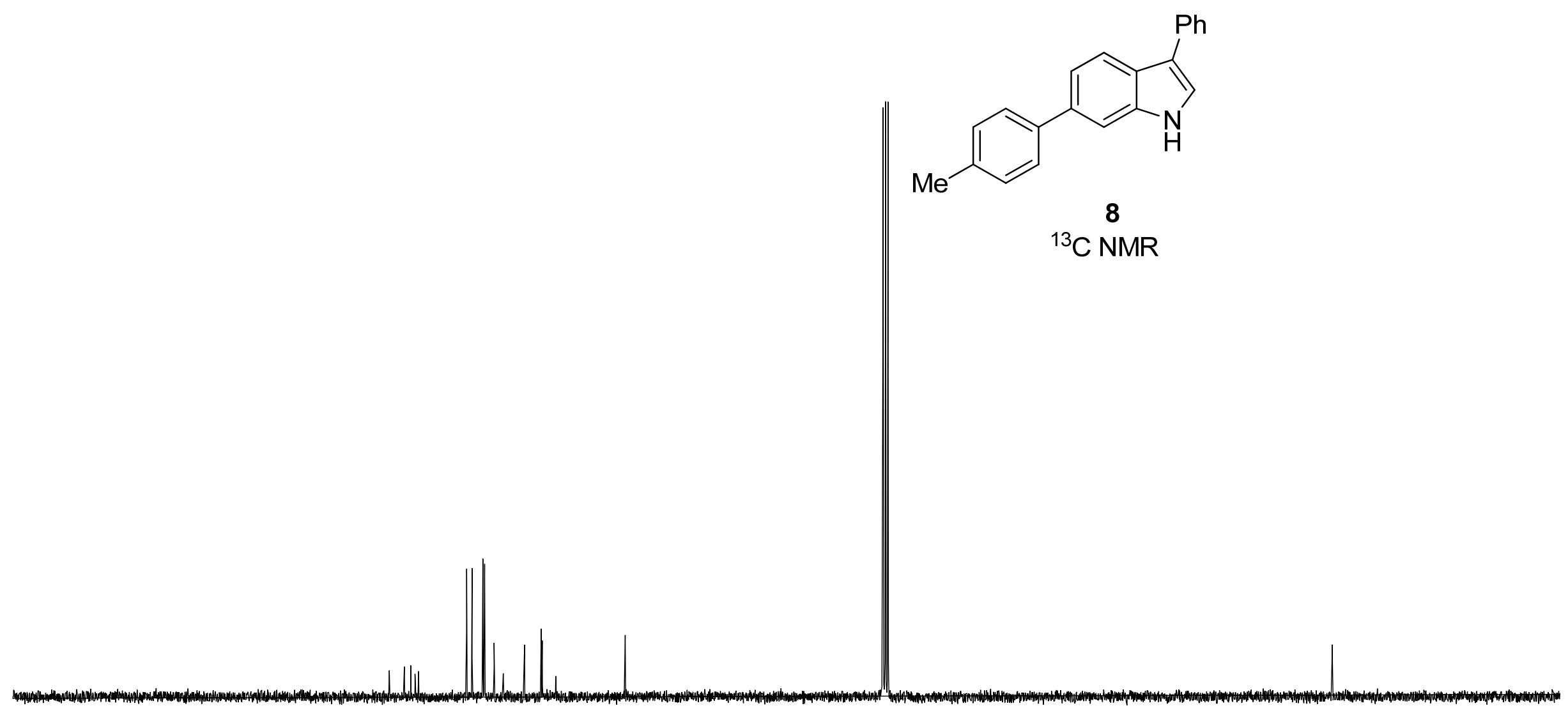

$\begin{array}{llllllllllllllllllllllllll}185 & 180 & 175 & 170 & 165 & 160 & 155 & 150 & 145 & 140 & 135 & 130 & 125 & 120 & 115 & 110 & 105 & 100 & 95 & 90 & 85\end{array}$ 